\title{
Entire Solutions \\ for Bistable Lattice Differential Equations with Obstacles
}

\author{
A. Hoffman $^{\text {a }}$, H. J. Hupkes ${ }^{\text {b,* }}$, E. S. Van Vleck ${ }^{\mathrm{c}}$

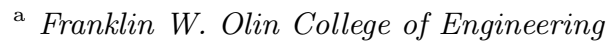 \\ 1000 Olin Way; Needham, MA 02492; USA \\ Email: aaron.hoffman@olin.edu \\ b Mathematisch Instituut - Universiteit Leiden \\ P.O. Box 9512; 2300 RA Leiden; The Netherlands \\ Email: hhupkes@math.leidenuniv.nl \\ c Department of Mathematics - University of Kansas \\ 1460 Jayhawk Blvd; Lawrence, KS 66045; USA \\ Email: erikvv@ku.edu
}

\begin{abstract}
We consider scalar lattice differential equations posed on square lattices in two space dimensions. Under certain natural conditions we show that wave-like solutions exist when obstacles (characterized by "holes") are present in the lattice. Our work generalizes to the discrete spatial setting the results obtained in [9] for the propagation of waves around obstacles in continuous spatial domains. The analysis hinges upon the development of sub and super-solutions for a class of discrete bistable reaction-diffusion problems and on a generalization of a classical result due to Aronson and Weinberger that concerns the spreading of localized disturbances.
\end{abstract}

AMS 2010 Subject Classification: 34K31, 37L15.

Key words: travelling waves, multi-dimensional lattice differential equations, obstacles, sub and super-solutions.

\section{Introduction}

Consider a subset $\Lambda \subset \mathbb{Z}^{2}$ that results after removing a finite (possibly zero) number of points from the standard square grid. Write $\operatorname{int}(\Lambda) \subset \Lambda$ for the collection of grid points for which all four nearest neighbours are also included in $\Lambda$ and write $\partial \Lambda=\Lambda \backslash \operatorname{int}(\Lambda)$ for the remaining points, which can be interpreted as the boundary of $\Lambda$. Fix a detuning parameter $0<a<1$ and consider the bistable nonlinearity $g(u)=u(1-u)(u-a)$.

In this paper we are interested in the scalar lattice differential equation (LDE)

$$
\dot{u}_{i j}(t)=u_{i+1, j}(t)+u_{i, j+1}(t)+u_{i-1, j}(t)+u_{i, j-1}(t)-4 u_{i j}(t)+g\left(u_{i j}(t)\right), \quad(i, j) \in \operatorname{int}(\Lambda),
$$

${ }^{*}$ Corresponding author.

Preprint submitted to ...

April 22, 2019 
augmented by rules on $\partial \Lambda$ that ensure that the homogeneous states $u \equiv 0, u \equiv a$ and $u \equiv 1$ are equilibria for the full problem. We encourage the reader to think of this system as the discrete analogue of the scalar Nagumo PDE

$$
\partial_{t} u(x, y, t)=\partial_{x x} u(x, y, t)+\partial_{y y} u(x, y, t)+g(u(x, y, t)), \quad(x, y) \in \Omega,
$$

posed on an exterior domain $\Omega=\mathbb{R}^{2} \backslash K$ for some compact (possibly empty) obstacle $K$, with Neumann boundary conditions on $\partial \Omega$.

In the unobstructed case $\Lambda=\mathbb{Z}^{2}$, it is known that (1.1) admits planar travelling wave solutions

$$
u_{i j}(t)=\Phi\left(i \sigma_{h}+j \sigma_{v}+c t\right), \quad \Phi(-\infty)=0, \quad \Phi(\infty)=1,
$$

which propagate with speed $-c$ in the direction $\left(\sigma_{h}, \sigma_{v}\right)$ with a fixed monotone wave profile $\Phi$. Our goal here is to show that these unobstructed waves persist in an appropriate sense, after removing points from $\mathbb{Z}^{2}$. In particular, we give conditions under which (1.1) with $\Lambda \neq \mathbb{Z}^{2}$ admits so-called entire asymptotic plane-wave solutions. Such solutions are defined for all $t \in \mathbb{R}$ and satisfy the temporal limits

$$
\lim _{|t| \rightarrow \infty} \sup _{(i, j) \in \Lambda}\left|u_{i j}(t)-\Phi\left(i \sigma_{h}+j \sigma_{v}+c t\right)\right|=0 .
$$

As such, the present work can be seen as a direct (partial) generalization of the results concerning the obstructed PDE (1.2) that were obtained by Berestycki, Hamel and Matano in the landmark paper [9].

Viewed from a dynamical system perspective, the limits (1.4) suggest that $u(t)$ can be seen as a homoclinic excursion to and from an unobstructed travelling wave, with large transients when the wave front meets the obstacle. In particular, the wave-like solutions constructed in the present paper can be seen in the wider context of so-called transition fronts, which can roughly be defined as global in time solutions for which the width of the interfacial region connecting the limiting values is uniformly bounded in time. This nomenclature allows classical travelling waves and more general wave-like solutions to be discussed within a common framework. Besides the work [9] mentioned above, results concerning the existence of transition fronts have appeared in a wide range of settings, including diffusive random media [39], reaction-diffusion-advection PDEs 7] and time-dependent reaction-diffusion PDEs [8]. As far as we know however, the present paper is the first that features transition fronts for LDEs in higher space dimensions.

\section{Reaction-Diffusion Problems}

The discrete and continuous Nagumo systems (1.1)-(1.2) can both be seen as phenomenological models in which two stable equilibria compete for dominance in a spatial domain. In modelling contexts one often thinks of these equilibria as representing material phases or biological species. The competition is caused by the opposing dynamical effects of the reaction and diffusion terms present in (1.1)-(1.2). Indeed, both equations feature a thresholding nonlinearity that promotes high frequencies together with a diffusion operator that attenuates them. The main questions center on how the long-term behavior of these reaction-diffusion systems is impacted by the balance between these dynamical features.

In the past, the PDE (1.2) has served as a prototype system for the understanding of many basic concepts at the heart of dynamical systems theory, including the existence and stability of planar travelling waves and the study of obstacles. Multi-component versions of (1.2) such as the GrayScott model [20] play an important role in the formation of patterns, generating spatially periodic structures from equilibria that destabilize through Turing bifurcations.

More recently, spatially discrete systems such as (1.1) have started to attract an increasing amount of attention. Dramatic increases in computer power have made these systems considerably more accessible and have clearly demonstrated that discrete models can capture dynamical behaviour that their continuous counterparts can not. Understanding the causes and consequences of these 
differences is a major theme that continues to drive researchers in this area, motivated by both mathematical and practical considerations.

On the mathematical side, the shift from the PDE (1.2) with $\Omega=\mathbb{R}^{2}$ to the LDE (1.1) with $\Lambda=\mathbb{Z}^{2}$ breaks two important symmetries, namely the translational invariance and spatial isotropy of $\mathbb{R}^{2}$. In the sequel two fundamental consequences of these broken symmetries are encountered. In addition, the discrete Laplacian in (1.1) is a bounded operator while the continuous Laplacian in (1.2) is unbounded. This requires the use of delicate techniques when comparing the spectral and dynamical properties of these two operators, as discussed in [4].

On the practical side, many physical and biological systems have a discrete spatial structure. It is hence important to develop mathematical modelling tools that can incorporate such structures effectively. Indeed, genuinely discrete phenomena such as phase transitions in Ising models [5] , crystal growth in materials [1], propagation of action potentials in myelinated nerve fibers [6] and phase mixing in martensitic structures [40] have all been modelled using equations similar to (1.1). We expect this list to get longer over time as the available mathematical techniques for discrete systems are improved.

Finally, we remark that the LDE (1.1) arises as the standard finite difference spatial discretization of the PDE (1.2). As such, the study of (1.1) and its variants provides information on the impact of discretization schemes and is therefore of importance in the field of numerical analysis. In order to faithfully replicate the PDE behavior in numerical simulations, the vested interest here is to actually suppress as best as possible the novel features appearing in discrete systems. Such issues are explored in 2 , 15, 26]. In this paper we take the neutral perspective that the discrete model is set and we seek to understand its behavior in its own right.

\section{Existence of Waves}

In the unobstructed PDE (1.2) with $\Omega=\mathbb{R}^{2}$, the balance between diffusion and reaction is resolved through the formation of planar travelling waves

$$
u(x, y, t)=\Phi\left(x \sigma_{h}+y \sigma_{v}+c t\right) ; \quad \Phi(-\infty)=0, \quad \Phi(\infty)=1,
$$

which form the skeleton of the global dynamics [1]. These waves can be thought of as a mechanism of transport in which the fitter species or more energetically favourable phase invades the spatial domain. The existence of these waves can be established via phase-plane analysis [19], since the wave profile necessarily satisfies the planar ODE

$$
c \Phi^{\prime}=\Phi^{\prime \prime}+g(\Phi) .
$$

Here we have assumed the normalization $\sigma_{h}^{2}+\sigma_{v}^{2}=1$. Notice that the underlying spatial dimension and the direction $\left(\sigma_{h}, \sigma_{v}\right)$ are not visible in the travelling wave ODE (1.6), which means that existence results in one spatial dimension can easily be transferred to higher spatial dimensions in a radially symmetric fashion.

By contrast, substitution of the discrete travelling wave Ansatz (1.3) into the LDE (1.1) with $\Lambda=\mathbb{Z}^{2}$ leads to the mixed type functional differential equation (MFDE)

$$
c \Phi^{\prime}(\xi)=\Phi\left(\xi+\sigma_{h}\right)+\Phi\left(\xi-\sigma_{h}\right)+\Phi\left(\xi+\sigma_{v}\right)+\Phi\left(\xi-\sigma_{v}\right)-4 \Phi(\xi)+g(\Phi(\xi)) .
$$

The broken translational invariance is manifested by the fact that the wavespeed $c$ appears in (1.7) in a singular fashion. This leads to the phenomenon of propagation failure, in which a sufficient energy difference between the two stable equilibrium states is needed for propagation of waves, together with the appearance of step-like wave solutions [5, 6, 17, 33].

The broken spatial isotropy is manifested by the explicit presence of the propagation direction $\left(\sigma_{h}, \sigma_{v}\right)$ in (1.7). This leads to direction-dependent wave speeds, wave forms and even pinning regions. In particular, waves can fail to propagate in certain directions that resonate with the lattice whilst 
travelling freely in others. This phenomenon has been studied when the bistable nonlinearity is piecewise linear via classical analysis [12], as well as for more general nonlinearities via numerics [16, 30], homoclinic-unfolding [36] and center manifold analysis [25].

\section{Stability of Waves}

While existence results for travelling wave solutions to the PDE (1.2) with $\Omega=\mathbb{R}^{2}$ do not depend on the spatial dimension, stability results do. In fact, it has long been known [18] that travelling fronts for one dimensional versions of (1.2) are nonlinearly stable under small perturbations, provided one allows for a small phase shift in the wave. Results in four or more dimensional problems have also been available for some time [41]. However, the first stability result in the critical case of two spatial dimensions was only obtained relatively recently by Kapitula [32].

The key feature that complicates the stability analysis of plane waves in two dimensions is that the interface, by which we mean a level set of the function $u(x, y, t)=\Phi(x+c t)$, is no longer localized in space at a point as it is in one dimension, but rather spread out over an entire line. This means that a phase shift is a big perturbation from the perspective of $L^{p}(p<\infty)$ : the difference $\Phi(x+\tau)-\Phi(x)$ does not live in $L^{p}$ for any $\tau \neq 0$ (with $p<\infty$ ). Thus it is not unreasonable to expect that a localized perturbation does not lead to a phase shift. On the other hand, one must now be concerned with long wave deformations of the interface in the direction transverse to that of propagation. Such deformations manifest themselves as curves of essential spectrum that touch the origin. This typically leads to slow algebraic decay at the linear level, which in the two dimensional case is notoriously difficult to control.

There are two main approaches towards establishing the stability of travelling waves. The first is based on spectral methods, Green's functions and bootstrapping or fixed-point arguments. This approach was developed by Kapitula [32] for the planar unobstructed PDE (1.2). Very recently [24], we were able to extend it to the planar unobstructed LDE (1.1), thereby generalizing earlier work by Bates and Chen [3] featuring a four-dimensional non-local setting. The advantage of this approach is that only weak spectral assumptions need to be imposed on the underlying system. On the other hand, such methods typically employ rather crude estimates on the nonlinear terms and hence yield rather weak estimates for the basin of attraction.

The second approach is based on comparison principles and yields stronger estimates for the basin of attraction, at the price of requiring more structure on the underlying system. The inclusion of obstacles in (1.1)-(1.2) is a rather strong perturbation from the unobstructed systems, so it is not surprising that comparison principles are the method of choice in the present context.

We remark that comparison principles were used in an early paper [41] to prove the stability of waves in an unobstructed four dimensional version of the PDE (1.2). Here the algebraic decay of the interfacial deformations described above is rather fast, considerably easing the analysis. However, besides the partial results in [34], the first successful use of the comparison principle in two dimensions was actually a byproduct of the analysis [9] on which this paper is based. It is therefore not surprising that part of our work here can be seen as a companion paper to [24], in the sense that we give an alternative proof of the nonlinear stability of travelling waves to the unobstructed planar LDE (1.1) with $\Lambda=\mathbb{Z}^{2}$.

\section{The Program}

As mentioned above, our contribution in this paper is to extend some of the main results in [9] to the spatially discrete setting. In fact, the main steps that underpin our arguments here closely mimic the program developed in [9]. In the remainder of this introduction we therefore describe this

program in some detail, explaining the achievements in [9] and the technical modifications required to extend them to the discrete setting.

Let us therefore consider either (1.1) or (1.2), with $\Lambda \neq \mathbb{Z}^{2}$ or $\Omega \neq \mathbb{R}^{2}$. One starts by considering the unobstructed wave when it is still far away from the obstacle, but moving towards it. As time 
progresses, the wave scatters from the obstacle and the goal is to prove that the wave eventually recovers its shape. To this end, one can distinguish the following three temporal regimes and study them separately:

(i) the pre-interaction regime in which the obstacle is so far away from the wave front that the obstacle essentially sees only an equilibrium solution;

(ii) the interaction regime in which the wave front and the obstacle interact strongly, producing a large transient; and finally,

(iii) the post-interaction regime in which the wave front is again far from the obstacle.

In order to construct the desired entire asymptotic plane wave solution, one roughly needs the following ingredients:

(i) a stability result which guarantees that the plane wave doesn't change much in the preinteraction regime;

(ii) a bound showing that the transient produced during the interaction regime is spatially localized;

(iii-A) a result that describes the final state of compact subsets of the medium, long after the wave front has passed through the region and the transients have died down; and finally

(iii-B) powerful estimates on the basin of attraction of the travelling planar wave that can accommodate the large spatially localized transients generated during the interaction regime.

\section{Pre-interaction regime}

Continuous Setting The main task in this step is to prove that the asymptotic requirement

$$
\lim _{t \rightarrow-\infty} \sup _{(x, y) \in \Omega}|u(x, y, t)-\Phi(x+c t)|=0,
$$

with $\Phi$ as in (1.6), fixes a unique solution to the obstructed PDE (1.2) that is defined for all $t \in \mathbb{R}$. The arguments used to achieve this in [9] are all one-dimensional in nature, in the sense that the sub and super-solutions that are used in the construction of $u$ depend only on the coordinate $x$ parallel to the direction of the wave.

In particular, a well-known squeezing technique is employed that goes back to the classic work of Fife and McLeod 19]. Roughly speaking, this technique allows additive initial perturbations to wave-like structures to be traded off for an asymptotic phase offset. Since this general principle lies at the heart of many other arguments in this paper, we discuss it here in some detail.

For the unobstructed PDE (1.2) with $\Omega=\mathbb{R}^{2}$, the squeezing mechanism can be exhibited by constructing a super-solution of the form

$$
u(x, y, t)=\Phi(x+c t+Z(t))+z(t),
$$

with $z$ decreasing from $z_{0}$ to 0 and with $Z$ increasing from 0 to $Z_{\infty}$. To better understand the crucial relationship between the asymptotic phaseshift $Z_{\infty}$ and the additive perturbation $z$, we note that the super-solution residual $\mathcal{J}=u_{t}-u_{x x}-g(u)$ is given by

$$
\mathcal{J}=\dot{Z} \Phi^{\prime}+\dot{z}+g(\Phi)-g(\Phi+z) .
$$

Close to the interface, the term $g(\Phi)-g(\Phi+z) \sim-g^{\prime}(\Phi) z$ is negative and must be dominated by the positive term $\dot{Z} \Phi^{\prime}$. This requires that $\dot{Z}$ dominate $z$ and $\dot{z}$. On the other hand, close to the 
spatial limits $\Phi \rightarrow 0$ and $\Phi \rightarrow 1$ we have $g^{\prime}(\Phi)<0$, so this regime requires $z$ to dominate $\dot{z}$. These observations allow us to define $z(t)$ as a slowly decaying exponential, which gives the relation

$$
Z_{\infty} \sim \int_{0}^{\infty} z(t) d t \sim z_{0}
$$

between the asymptotic phase shift and the size of the initial perturbation.

In one spatial dimension, this technique (along with others) has been used to establish the nonlinear stability of travelling waves under asymptotic phase shifts. In our present context, a slight variant is used in [9] to establish the uniqueness of the entire solution $u$ for (1.2) with (1.8) that was mentioned above.

On the other hand, the existence of this solution $u$ is harder to establish. The main procedure in [9] is to split the plane $\mathbb{R}^{2}$ into two half-planes along a vertical line, with the obstacle contained entirely in one of the half-planes. One can then focus on time regimes where the bulk of the incoming wave is contained in the obstacle-free half-plane. Following ideas in [21, 23], one can then trap the desired entire solution $u$ between the sum and the difference of two counter-propagating wave fronts. This allows the use of a limiting argument to show that the solution $u$ actually exists.

Discrete Setting The main ideas of the PDE analysis described above can be carried over to the LDE setting without major complications. Indeed, arguments based on the comparison principle are well-developed for systems posed on one-dimensional lattices; see for example [14, 29]. Care must however be taken when studying the counter-propagating wave fronts, as the discrete Laplacian causes cross-talk between the two-half planes that needs to be carefully controlled. This analysis is carried out in $\S 6$.

\section{Interaction regime}

Continuous Setting The main issue in this phase is to show that the large perturbations that arise when the wave front hits the obstacle are spatially localized. In particular, in [9] the authors show that whenever the obstacle $\mathbb{R}^{2} \backslash \Omega$ is bounded, the entire solution $u$ that satisfies the temporal requirement (1.8) also admits the spatial limits

$$
\lim _{|x|+|y| \rightarrow \infty}|u(x, y, t)-\Phi(x+c t)|=0,
$$

locally uniformly in $t$.

For the transverse direction $y \rightarrow \infty$, this result can be established using standard parabolic estimates, since the obstacle can effectively be pushed to $y=-\infty$ and hence ignored in the limit. The analysis for $x \rightarrow-\infty$ is more subtle and does need to take the obstacle into account. In order to do this, two branches of modified nonlinearities $g_{\delta}^{ \pm}$are constructed in [9] with the property that $g_{\delta}^{-} \leq g \leq g_{\delta}^{+}$. These modified nonlinearities have zeroes at $g^{-}(-\delta)=g^{+}(\delta)=0$ and $g^{-}(1-\delta)=$ $g^{+}(1+\delta)=0$. This allows travelling waves for the unobstructed problem with nonlinearities $g^{ \pm}$to be effectively used as sub and super-solutions for the original obstructed problem.

Discrete Setting The parabolic estimates and limiting arguments described above can be readily transferred to the discrete setting; see $\S 7$. On the other hand, special care needs to be taken in the construction of the modified nonlinearities $g_{\delta}^{ \pm}$, which we perform in $\S 3$. This is related to the fact that the existence of travelling waves $(c, \Phi)$ for unobstructed lattices hinges on a delicate analysis of the MFDE (1.7); see [35]. Together with our generalization of the classical spreading speed result, this requires smoothness on the part of the nonlinearities beyond that which was used in [9]. 


\section{Post-interaction regime - rest state}

Continuous Setting The first key result obtained in [9] for this phase is that one has the temporal convergence

$$
\lim _{t \rightarrow \infty} u(x, y, t) \rightarrow u_{\infty}(x, y),
$$

locally uniformly in $(x, y)$, where $u_{\infty}$ is a stationary solution to the obstructed PDE (1.2) that admits the spatial limits

$$
\lim _{|x|+|y| \rightarrow \infty} u_{\infty}(x, y)=1,
$$

together with Neumann boundary conditions on $\partial \Omega$.

Let us briefly comment on the interpretation of this spatial limit. A classical result due to Aronson and Weinberger [1, Thm. 5.3] for the unobstructed PDE (1.2) with $\Omega=\mathbb{R}^{2}$ states that an initial disturbance from the $u \equiv 0$ rest state that occupies a large area of the spatial domain and is large in amplitude, will eventually spread to fill out the entire spatial domain. The radial speed of this spreading is comparable to that of a travelling planar wave. In [9, Lem. 5.2] a similar result was established for the obstructed case $\Omega \neq \mathbb{R}^{2}$. In particular, the authors established that disturbances such as those described above continue to spread out in all directions, providing one restricts attention to regions of space that are sufficiently far away from the obstacle. This provides a mechanism by which the disturbance caused by the incoming wave can bend around the obstacle. In particular, the favoured state $u \equiv 1$ invades the domain in an asymptotic sense.

It is clear that the homogeneous equilibrium $u_{\infty} \equiv 1$ is a candidate for the temporal limit (1.13) discussed above. It is however by no means clear that this is the only candidate. The second key result in [9] gives geometric conditions on the obstacle $\mathbb{R}^{2} \backslash \Omega$ that are sufficient to guarantee that in fact $u_{\infty} \equiv 1$. These conditions are satisfied if the obstacle is star-shaped or directionally convex, but are far from being sharp. For example, a recent result due to Bouhours [10] characterizes a class of admissible perturbations to such obstacles that still allow one to prove $u_{\infty} \equiv 1$.

Discrete Setting The main issue in the discrete setting is that an analogue of the classic Aronson and Weinberger spreading speed result described above is not readily available in the literature. This situation is remedied in $\S 4$, where we construct expanding sub-solutions based on the gluing together of planar travelling waves. The main obstruction here is that the speeds and profiles of these planar waves are direction-dependent, which prevents us from using a radially symmetric construction and requires a delicate balancing of angular dependent terms.

Naturally, such a spreading result can only be established if the wave speeds are strictly positive for every direction. In the discrete case, an important role is therefore reserved for the pinning phenomenon discussed above, which can block propagation in certain directions. In the present paper we avoid such complications and stay away from the pinning regime. This issue is discussed further in $\S 9$.

We also do not fully explore the issues concerning the geometry of the obstacle. We do however show in $\S 7$ that directionally convex obstacles force the identity $u_{\infty} \equiv 1$, which allows our results to be applied to an important class of non-empty bounded obstacles.

\section{Post-interaction regime - convergence to wave}

Continuous Setting In this final step of the program, the large transients generated in the interaction regime must be controlled in a frame that moves along with the unobstructed wave. As discussed above, this analysis leads naturally to a large basin nonlinear stability result for planar travelling wave solutions to the unobstructed PDE (1.2) with $\Omega=\mathbb{R}^{2}$. For presentation purposes, we will focus our discussion here on this unobstructed special case. Indeed, the inclusion of the obstacle merely adds technical complications that do not contribute to the understanding of the differences between the continuous and discrete frameworks. 
The main task is to construct a super-solution for (1.2) with $\Omega=\mathbb{R}^{2}$ of the form

$$
u(x, y, t)=\Phi(x+c t+\theta(y, t)+Z(t))+z(t),
$$

which adds transverse effects to the Ansatz (1.9) discussed earlier. As before, the function $z$ decreases from $z_{0}$ to 0 while $Z$ increases from 0 to $Z_{\infty}$. Both $z$ and $Z$ should be thought of as small terms. By contrast, the new function $\theta$ should be allowed to be arbitrarily large at $t=0$, provided that it is localized in the sense $\theta(\cdot, 0) \in L^{2}$ and that it decays to zero as $t \rightarrow \infty$ uniformly in $y$. This function controls deformations of the wave interface in the transverse direction.

We note that any localized initial perturbation from the wave can be dominated by the initial condition in (1.15) by choosing $z_{0}$ positive and as small as we wish, at the cost of a larger value for $\|\theta(\cdot, 0)\|_{L^{2}}$. Assuming for the moment that $Z_{\infty}$ scales with $z_{0}$, this freedom implies that we can dominate the transients caused by such a perturbation by a family of super-solutions that have arbitrarily small asymptotic phase offsets $Z_{\infty}$. A similar argument with sub-solutions then establishes the convergence to the planar wave without any asymptotic phase shift.

The difference in behaviour between one and two spatial dimensions is hence caused by the extra transverse direction, along which perturbations can diffuse in a sense without causing a phase shift. The assumption that the initial transverse perturbation $\theta(\cdot, 0)$ is localized is crucial here. Indeed, if $\theta(\cdot, 0)$ is not localized but still very small, the perturbations can not only cause phase offsets of the underlying wave, but can also prevent solutions from converging to any translate of the wave at all [38].

We now proceed to discuss the choices made in [9] for the functions $\theta, Z$ and $z$, which ensure that (1.15) is indeed a super-solution with $Z_{\infty} \sim z_{0}$. As before, we write $\mathcal{J}=u_{t}-\Delta u-g(u)$ for the super solution residual. A short computation shows that we can split $\mathcal{J}$ into the three parts

$$
\mathcal{J}=\mathcal{J}_{\text {glb }}+\mathcal{J}_{\text {heat }}+\mathcal{J}_{\text {nl }}
$$

which with the shorthand $\xi=x+c t+\theta(y, t)+Z(t)$ can be written as

$$
\begin{aligned}
& \mathcal{J}_{\text {glb }}(x, y, t)=\dot{Z}(t) \Phi^{\prime}(\xi)+\dot{z}(t)+g(\Phi(\xi))-g(\Phi(\xi)+z(t)), \\
& \mathcal{J}_{\text {heat }}(x, y, t)=\Phi^{\prime}(\xi)\left(\partial_{t} \theta(y, t)-\partial_{y y} \theta(y, t)\right), \\
& \mathcal{J}_{\text {nl }}(x, y, t)=-\Phi^{\prime \prime}(\xi)\left[\partial_{y} \theta(y, t)\right]^{2} .
\end{aligned}
$$

Exactly as discussed earlier, one can ensure that $\mathcal{J}_{\mathrm{glb}}(x, y, t) \geq 0$ by picking $z(t)$ to be a slowly decaying exponential. This gives the desired proportionality $Z_{\infty} \sim z_{0}$. Since $\Phi^{\prime \prime}$ does not admit a sign, the two remaining terms $\mathcal{J}_{\text {heat }}$ and $\mathcal{J}_{\text {nl }}$ need to be treated together. The structure of $\mathcal{J}_{\text {heat }}$ strongly suggests a relation with the heat equation and that is precisely what is exploited in [9].

To set the stage, let us introduce the functions

$$
v(y, t)=e^{-y^{2} / 4 t}, \quad h(y, t)=\frac{1}{\sqrt{4 \pi t}} v(y, t)
$$

and remark that the one dimensional heat kernel $h(y, t)$ can be seen in an appropriate sense as the solution of the initial value problem

$$
\partial_{t} h=\partial_{y y} h, \quad h(y, 0)=\delta(y) .
$$

The choice for $\theta$ used in [9] is now given by

$$
\theta(y, t)=\beta(t+1)^{-2 \gamma^{-1}} v(y, \gamma(t+1)),
$$

in which $\gamma=\gamma(\beta) \gg \beta$ and $\beta \gg 1$ can be chosen to be arbitrarily large. In particular, the function $\theta$ can be seen as a modified heat-kernel where the diffusion is sped up by a factor $\gamma$ and the decay rate at the center $y=0$ is slowed down to $\beta(t+1)^{-2 \gamma^{-1}}$. The partial derivatives $\partial_{t} \theta, \partial_{y} \theta$ and $\partial_{y y} \theta$ can all be explicitly evaluated and yield rational functions of $t$ and $y$ multiplied by $v(y, t)$. Exploiting a uniform bound $\left|\Phi^{\prime \prime}(\xi)\right| \leq K \Phi^{\prime}(\xi)$, one can decouple $\xi$ from the pair $(y, t)$ and guarantee $\mathcal{J}_{\text {heat }}+\mathcal{J}_{\text {nl }} \geq 0$ by picking $\gamma$ appropriately. 
Discrete Setting Considerable modifications must be made to the procedure outlined above before it can be used in the discrete setting. To appreciate the difficulties involved, let us consider the discrete analogue of the super-solution Ansatz (1.15). Choosing $\left(\sigma_{h}, \sigma_{v}\right) \in \mathbb{Z}^{2}$, this can be written as

$$
u_{i j}(t)=\Phi\left(i \sigma_{h}+j \sigma_{v}+c t+\theta_{i \sigma_{v}-j \sigma_{h}}(t)+Z(t)\right)+z(t) .
$$

Writing $\mathcal{J}$ for the appropriate super-solution residual, we note that it can again be split up into three components $\mathcal{J}=\mathcal{J}_{\text {glb }}+\mathcal{J}_{\text {heat }}+\mathcal{J}_{\text {nl }}$ that are the discrete analogues of (1.17). For the presentation here it is only necessary to fully write out one of these terms. Indeed, introducing the shorthands $\xi=i \sigma_{v}+j \sigma_{h}+c t$ and $l=i \sigma_{v}-j \sigma_{h} \in \mathbb{Z}$, we restrict our attention to the component

$$
\begin{aligned}
\mathcal{J}_{\text {heat }}= & \Phi^{\prime}(\xi) \dot{\theta}_{l}(t)-\Phi^{\prime}\left(\xi+\sigma_{h}\right)\left(\theta_{l+\sigma_{v}}(t)-\theta_{l}(t)\right)-\Phi^{\prime}\left(\xi-\sigma_{h}\right)\left(\theta_{l-\sigma_{v}}(t)-\theta_{l}(t)\right) \\
& -\Phi^{\prime}\left(\xi-\sigma_{v}\right)\left(\theta_{l+\sigma_{h}}(t)-\theta_{l}(t)\right)-\Phi^{\prime}\left(\xi+\sigma_{v}\right)\left(\theta_{l-\sigma_{h}}(t)-\theta_{l}(t)\right),
\end{aligned}
$$

which is a sum of first differences in $\theta$, each multiplied by a different shifted version of $\Phi^{\prime}$. Since $\mathcal{J}_{\text {heat }}$ no longer factors as a positive function of $\xi$ times a parabolic operator acting on $\theta$, it is not at all clear how the approach described above for the continuous setting can be mimicked.

Our inspiration to deal with this challenge comes from the art of normal form transformations. In particular, we add four specially chosen auxilliary terms to the Ansatz (1.21). This replaces the shifted coefficients $\Phi^{\prime}\left(\xi \pm \sigma_{h}\right)$ and $\Phi^{\prime}\left(\xi \pm \sigma_{v}\right)$ that appear in $\mathcal{J}_{\text {heat }}$ by unshifted coefficients $\Phi^{\prime}(\xi)$, while generating additional terms that only involve higher order differences in $\theta$ or products of lower order differences.

This construction relies on solving linear inhomogeneous MFDEs. More specifically, the four new terms added to (1.21) can all be factorized as a first difference in $\theta$ multiplied by $\xi$-dependent functions $p_{h}^{ \pm}$and $p_{v}^{ \pm}$that satisfy

$$
\left[\mathcal{L}_{0} p_{\#}^{ \pm}\right](\xi)=\Phi^{\prime}\left(\xi \pm \sigma_{\#}\right)-\alpha_{\#}^{ \pm} \Phi^{\prime}(\xi), \quad \#=h, v .
$$

Here the linear operator $\mathcal{L}_{0}: W^{1, \infty}(\mathbb{R}, \mathbb{R}) \rightarrow L^{\infty}(\mathbb{R}, \mathbb{R})$ is given by

$$
\begin{gathered}
{\left[\mathcal{L}_{0} p\right](\xi)=-c p^{\prime}(\xi)+p\left(\xi+\sigma_{h}\right)+p\left(\xi+\sigma_{v}\right)+p\left(\xi-\sigma_{h}\right)+p\left(\xi-\sigma_{v}\right)-4 p(\xi)} \\
+g^{\prime}(\Phi(\xi)) p(\xi)
\end{gathered}
$$

and the constants $\alpha_{\#}^{ \pm}$are fixed by the requirement that the system is actually solvable. The details of this construction rely heavily on the linear Fredholm theory developed by Mallet-Paret [35]. In any case, let us remark here that $\mathcal{L}_{0}$ is related to the linearization of the travelling wave MFDE (1.7) around the solution $\Phi$. Indeed, we have $\mathcal{L}_{0} \Phi^{\prime}=0$, which means that we expect the asymptotics $p_{\#}^{ \pm}(\xi) \sim \xi \Phi^{\prime}(\xi)$ as $\xi \rightarrow \pm \infty$ as the right hand side of (1.24) is resonant.

It is worthwhile to pause here to fully understand the ramifications of our analysis up to this point. With the new terms, we obtain

$$
\begin{aligned}
\mathcal{J}_{\text {heat }}=\Phi^{\prime}(\xi) & {\left[\dot{\theta}_{l}(t)-\alpha_{h}^{+}\left(\theta_{l+\sigma_{v}}(t)-\theta_{l}(t)\right)-\alpha_{h}^{-}\left(\theta_{l-\sigma_{v}}(t)-\theta_{l}(t)\right)\right.} \\
& \left.-\alpha_{v}^{-}\left(\theta_{l+\sigma_{h}}(t)-\theta_{l}(t)\right)-\alpha_{v}^{+}\left(\theta_{l-\sigma_{h}}(t)-\theta_{l}(t)\right)\right]+ \text { h.o.t. },
\end{aligned}
$$

in which the higher order terms contain, amongst others, products of second order differences in $\theta$ and the functions $p_{\#}^{ \pm}(\xi)$. It is now tempting to try to proceed as in the continuous case, writing $h_{l}(t)$ for the solution of the initial value problem

$$
\begin{aligned}
\dot{h}_{l}(t)= & \alpha_{h}^{+}\left[h_{l+\sigma_{v}}(t)-h_{l}(t)\right]+\alpha_{h}^{-}\left[h_{l-\sigma_{v}}(t)-h_{l}(t)\right] \\
& \quad+\alpha_{v}^{-}\left[h_{l+\sigma_{h}}(t)-h_{l}(t)\right]+\alpha_{v}^{+}\left[h_{l-\sigma_{h}}(t)-h_{l}(t)\right], \\
h_{l}(0)= & \delta_{l, 0}
\end{aligned}
$$


and building $\theta$ from $h$ in a fashion similar to (1.20). For $\gamma \gg 1$, this choice would imply that $\sup _{l \in \mathbb{Z}}\left|\theta_{l}(t)\right|$ remains roughly constant over long periods of time, while $k$-th order differences in $\theta$ would roughly decay at the rate $t^{-k / 2}$.

There are however three important issues that complicate such an attempt. First of all, the heat-like problem (1.26) contains convective terms, in contrast to (1.19). These terms manifest themselves as $e^{ \pm i \omega \sigma_{\#}}-1=O(i \omega)$ contributions when Fourier-expanding the first differences in $\theta$ in the $l$-direction. When scaling time as in (1.20), one must therefore take care that only the diffusive effects are sped up, since the convective effects do not generate terms of a definite sign.

The second problem is that the $\xi$-dependent terms that behave as $\xi \Phi^{\prime}(\xi)$ can never be dominated by the signed terms proportional to $\Phi^{\prime}(\xi)$ that can be obtained by carefully exploiting (1.26). It is therefore necessary to use $\mathcal{J}_{\text {glb }}$ for this purpose. This implies that we would need $\mathcal{J}_{\text {glb }} \sim z(t) \sim t^{-1}$, since these troublesome terms come with second order differences in $\theta$. Unfortunately, this gives $Z(t) \sim \ln (t)$, destroying any hope of closing a stability argument.

Finally, the third problem is caused by the fact that (1.26) does not actually capture all the diffusive effects present in the problem. Indeed, second order differences in $\theta$ also generate $O\left(\omega^{2}\right)$ contributions in Fourier space. Naturally, this leads to trouble when trying to control the second order terms in (1.25), even if they are multiplied by functions proportional to $\Phi^{\prime}(\xi)$.

In order to solve the second and third problems, it is necessary to perform an additional step in the normal form inspired expansion. In particular, we add a large number of extra terms to the Ansatz (1.21) in order to explicitly control all second order differences in $\theta$ and all products of first order differences in $\theta$.

Although this operation is a bookkeeping nightmare, it serves two crucial purposes. First of all, it ensures that all diffusive effects are made visible. It is here that the link to our prior work [24] based on spectral techniques becomes apparent, since we recover a condition on the essential spectrum that ensures that the diffusion coefficient is non-zero. Furthermore, the decay rate of all remaining terms is pushed to at least $t^{-3 / 2}$, corresponding to third order differences in $\theta$. Since this latter function is integrable over $[1, \infty)$, these terms can be successfully absorbed into $\mathcal{J}_{\text {glb }}$.

In contrast to the continuous setting, we therefore see that the three separate components $\mathcal{J}_{\text {glb }}$, $\mathcal{J}_{\text {heat }}$ and $\mathcal{J}_{\text {nl }}$ of the super-solution residual need to be analysed together. In addition, one can no longer use decaying exponentials for the global functions $Z(t)$ and $z(t)$, which requires considerably more care to be taken at many points in the analysis. These issues are explored in detail in $\S 5$, which we consider to be the heart of this paper.

\section{Organization}

This paper is organized as follows. In $\S 2$ we state our main results along with their underlying assumptions. A comparison principle that is used throughout the paper is established in $\S 3$, along with asymptotic properties of planar travelling wave solutions to the unobstructed LDE. In $\S 4$ we establish a result similar in spirit to [1, Thm. 5.3], stating that large disturbances from the zero rest state fill the entire unobstructed lattice $\mathbb{Z}^{2}$, provided the support of the initial condition is large enough. Sub and super-solutions providing a large basin of attraction for the waves mentioned above are constructed in $\S 5$. In $\S 6$ we focus on the behavior of the obstructed LDE and establish the existence of an entire solution that resembles an unobstructed wave as $t \rightarrow-\infty$. The behaviour of this entire solution in several limiting regimes is studied in $\S 7$. The proof of our main result is presented in $\S 8$, followed by a discussion in $\S 9$.

Reader's guide In order to prevent disruptions to the flow of ideas, the proof of many of our results is deferred to the second part of their respective sections. Constants that are used across different sections are given names, constants used only within a section are given numbers, while constants used only within proofs are given primes. We also use primes to denote derivatives, but trust that no confusion shall result. 
Acknowledgments Hoffman acknowledges support from the NSF (DMS-1108788). Hupkes acknowledges support from the Netherlands Organization for Scientific Research (NWO). Van Vleck acknowledges support from the NSF (DMS-1115408).

\section{Main Results}

In this section we outline our two main results. The first result concerns a two-dimensional obstacle free lattice and is essentially a stability result for travelling planar fronts. The second result shows that these travelling planar fronts persist in an appropriate sense after perturbing the lattice by removing grid points.

\subsection{Homogeneous Lattice}

Let us first consider the spatially homogeneous LDE

$$
\dot{u}_{i j}(t)=\left[\Delta^{+} u(t)\right]_{i j}+g\left(u_{i j}(t)\right),
$$

in which the plus-shaped discrete Laplacian acts on a planar sequence $u \in \ell^{\infty}\left(\mathbb{Z}^{2} ; \mathbb{R}\right)$ as

$$
\left[\Delta^{+} u\right]_{i j}=u_{i+1, j}+u_{i, j+1}+u_{i-1, j}+u_{i, j-1}-4 u_{i j} .
$$

The conditions on the nonlinearity $g$ that we will need are summarized in the following assumption.

(Hg) The nonlinearity $g: \mathbb{R} \rightarrow \mathbb{R}$ is $C^{2}$-smooth and has a bistable structure, in the sense that there exists a constant $0<a<1$ such that we have

$$
g(0)=g(a)=g(1)=0, \quad g^{\prime}(0)<0, \quad g^{\prime}(1)<0,
$$

together with

$$
g(u)<0 \text { for } u \in(0, a) \cup(1, \infty), \quad g(u)>0 \text { for } u \in(-\infty,-1) \cup(a, 1) .
$$

We note that the results in [35] guarantee that (2.1) has planar travelling wave solutions for every possible direction of propagation. The key requirement in our next assumption is that all these waves travel at a strictly positive speed.

$(\mathrm{H} \Phi)$ For each angle $\zeta \in[0,2 \pi]$, there exists a wave speed $c_{\zeta}>0$ and a profile $\Phi_{\zeta} \in C^{1}(\mathbb{R}, \mathbb{R})$ that satisfies the limits

$$
\lim _{\xi \rightarrow-\infty} \Phi_{\zeta}(\xi)=0, \quad \lim _{\xi \rightarrow+\infty} \Phi_{\zeta}(\xi)=1
$$

and yields a solution to the homogeneous LDE (2.1) upon writing

$$
u_{i j}(t)=\Phi_{\zeta}\left(i \cos \zeta+j \sin \zeta+c_{\zeta} t\right) .
$$

For the remainder of this subsection, we fix a specific angle $\zeta_{*}$ for which $\tan \zeta_{*} \in \mathbb{Q}$. This allows us to pick a pair $\left(\sigma_{h}, \sigma_{v}\right) \in \mathbb{Z}^{2}$ with $\operatorname{gcd}\left(\sigma_{h}, \sigma_{v}\right)=1$, for which we have the identity

$$
\left[\sigma_{h}^{2}+\sigma_{v}^{2}\right]^{1 / 2}\left(\cos \zeta_{*}, \sin \zeta_{*}\right)=\left(\sigma_{h}, \sigma_{v}\right) .
$$

As a consequence of $(H \Phi)$, there exists a wave speed $c>0$ and a wave profile $\Phi \in C^{1}(\mathbb{R}, \mathbb{R})$ that satisfies the limits (2.5), so that the LDE (2.1) admits a solution

$$
u_{i j}(t)=\Phi\left(i \sigma_{h}+j \sigma_{v}+c t\right) .
$$


We emphasize here that the pair $(c, \Phi)$ is a rescaled version of the pair $\left(c_{\zeta_{*}}, \Phi_{\zeta_{*}}\right)$. We choose to use this rescaling here for the benefit of the discussion below.

A standard approach towards establishing the stability of the wave solution (2.8) under the nonlinear dynamics of the LDE (2.1) is to consider the linear variational problem

$$
\dot{v}_{i j}(t)=\left[\Delta^{+} v(t)\right]_{i j}+g^{\prime}\left(\Phi\left(i \sigma_{h}+j \sigma_{v}+c t\right)\right) v_{i j}(t) .
$$

As can be seen, the linear operator on the right hand side of this system does not have constant coefficients and hence cannot be diagonalized via Fourier transform. It can however be partially diagonalized if one takes the Fourier transform in the direction that is perpendicular to the propagation of the wave, i.e., upon taking $i \sigma_{h}+j \sigma_{v}=$ constant. As explained in [24], one arrives for each transverse spatial frequency $\omega \in[-\pi, \pi]$ at an LDE posed on a one dimensional lattice that is parallel to the direction of propagation. This LDE is given by

$$
\begin{gathered}
\dot{v}_{n}(t)=e^{i \sigma_{v} \omega} v_{n+\sigma_{h}}(t)+e^{-i \sigma_{h} \omega} v_{n+\sigma_{v}}(t)+e^{-i \sigma_{v} \omega} v_{n-\sigma_{h}}(t)+e^{i \sigma_{h} \omega} v_{n-\sigma_{v}}(t)-4 v_{n}(t) \\
+g^{\prime}(\Phi(n+c t)) v_{n}(t),
\end{gathered}
$$

in which $n=i \sigma_{h}+j \sigma_{v} \in \mathbb{Z}$. As explained in detail in [27, §2], there is a close relationship between the Green's function for the LDE (2.10) and the linear operators

$$
\mathcal{L}_{\omega}: W^{1, \infty}(\mathbb{R}, \mathbb{C}) \rightarrow L^{\infty}(\mathbb{R}, \mathbb{C}), \quad \omega \in[-\pi, \pi],
$$

that act as

$$
\begin{gathered}
{\left[\mathcal{L}_{\omega} p\right](\xi)=-c p^{\prime}(\xi)+e^{i \sigma_{v} \omega} p\left(\xi+\sigma_{h}\right)+e^{-i \sigma_{h} \omega} p\left(\xi+\sigma_{v}\right)+e^{-i \sigma_{v} \omega} p\left(\xi-\sigma_{h}\right)+e^{i \sigma_{h} \omega} p\left(\xi-\sigma_{v}\right)} \\
-4 p(\xi)+g^{\prime}(\Phi(\xi)) p(\xi) .
\end{gathered}
$$

The formal adjoints of these operators are written as

$$
\mathcal{L}_{\omega}^{*}: W^{1, \infty}(\mathbb{R}, \mathbb{C}) \rightarrow L^{\infty}(\mathbb{R}, \mathbb{C}), \quad \omega \in[-\pi, \pi],
$$

and act as

$$
\begin{gathered}
{\left[\mathcal{L}_{\omega}^{*} q\right](\xi)=c q^{\prime}(\xi)+e^{-i \sigma_{v} \omega} q\left(\xi-\sigma_{h}\right)+e^{+i \sigma_{h} \omega} q\left(\xi-\sigma_{v}\right)+e^{+i \sigma_{v} \omega} q\left(\xi+\sigma_{h}\right)+e^{-\sigma_{h} \omega} q\left(\xi+\sigma_{v}\right)} \\
-4 q(\xi)+g^{\prime}(\Phi(\xi)) q(\xi) .
\end{gathered}
$$

Indeed, the designation of formal adjoint is justified by an easy computation, which shows that

$$
\int_{-\infty}^{\infty} q^{*}(\xi)\left[\mathcal{L}_{\omega} p\right](\xi) d \xi=\int_{-\infty}^{\infty}\left[\mathcal{L}_{\omega}^{*} q\right](\xi)^{*} p(\xi) d \xi
$$

holds for all pairs $p, q \in W^{1, \infty}(\mathbb{R}, \mathbb{C})$.

In view of the fact that the shifts are all integer valued, an easy computation shows that

$$
e_{-2 \pi i \ell}\left(\mathcal{L}_{\omega}-\lambda\right) e_{2 \pi i \ell}=\mathcal{L}_{\omega}-2 \pi i \ell c-\lambda
$$

for all $\lambda \in \mathbb{C}$ and $\ell \in \mathbb{Z}$, in which the exponential shift operator $e_{\nu}$ is defined by

$$
\left[e_{\nu} v\right](\xi)=e^{\nu \xi} v(\xi)
$$

In particular, for all $\omega \in[-\pi, \pi]$, the spectrum of $\mathcal{L}_{\omega}$ is invariant under the operation $\lambda \mapsto \lambda+2 \pi i c$.

As discussed in detail in [24], the operator $\mathcal{L}_{0}$ encodes stability properties of the wave $(c, \Phi)$ under perturbations that are constant in the direction transverse to propagation. In any case, it is easy to verify that $\mathcal{L}_{0} \Phi^{\prime}=0$ holds. The results in [35] show that $\mathcal{L}_{0}$ is a Fredholm operator of index 
zero with a one-dimensional kernel. In addition, these results give the existence of a strictly positive bounded function $\Psi \in C^{1}(\mathbb{R}, \mathbb{R})$ for which

$$
\mathcal{L}_{0}^{*} \Psi=0, \quad \int_{\mathbb{R}} \Psi(\xi) \Phi^{\prime}(\xi) d \xi=1 .
$$

In view of the characterization

$$
\operatorname{Range}\left(\mathcal{L}_{0}\right)=\left\{f \in L^{\infty}(\mathbb{R} ; \mathbb{R}): \int_{\mathbb{R}} \Psi(\xi) f(\xi) d \xi=0\right\},
$$

this directly implies that $\lambda=0$ is a simple eigenvalue of $\mathcal{L}_{0}$.

The next result, taken from [24], show how this simple eigenvalue behaves as $\omega$ is varied. It basically states that there is a branch of simple eigenvalues $\lambda_{\omega}$ and eigenfunctions $\phi_{\omega}$ for the operators $\mathcal{L}_{\omega}$ with $\omega \approx 0$. With the exception of simple eigenvalues at $\lambda_{\omega}+2 \pi i c \mathbb{Z}$, the spectrum of $\mathcal{L}_{\omega}$ lies to the left of the line $\operatorname{Re} \lambda=-\beta$.

Proposition 2.1 ([24, Prop. 2.1]). Consider the unobstructed LDE (2.1) and suppose that (Hg) is satisfied. Pick a direction $\zeta_{*} \in \mathbb{R}$ for which $\tan \zeta_{*} \in \mathbb{Q}$ and suppose that the requirements stated in (HФ) all hold, but only for the angle $\zeta_{*}$.

Then there exists a constant $\delta_{\omega}>0$ together with pairs

$$
\left(\lambda_{\omega}, \phi_{\omega}\right) \in \mathbb{C} \times W^{1, \infty}(\mathbb{R}, \mathbb{C}),
$$

defined for each $\omega \in\left(-\delta_{\omega}, \delta_{\omega}\right)$, such that the following holds true.

(i) There exists $\beta>0$ such that for all $\omega \in\left(-\delta_{\omega}, \delta_{\omega}\right)$, the operator $\mathcal{L}_{\omega}-\lambda$ is invertible as a map from $W^{1, \infty}(\mathbb{R}, \mathbb{C})$ into $L^{\infty}(\mathbb{R}, \mathbb{C})$ for all $\lambda \in \mathbb{C}$ that have $\operatorname{Re} \lambda \geq-\beta$ and for which $\lambda-\lambda_{\omega} \notin 2 \pi i c \mathbb{Z}$.

(ii) The only nontrivial solutions $p \in W^{1, \infty}(\mathbb{R}, \mathbb{C})$ of $\left(\mathcal{L}_{\omega}-\lambda_{\omega}\right) p=0$ are $p=\phi_{\omega}$ and scalar multiples thereof.

(iii) For all $\omega \in\left(-\delta_{\omega}, \delta_{\omega}\right)$, the equation $\left(\mathcal{L}_{\omega}-\lambda_{\omega}\right) v=\phi_{\omega}$ does not admit a solution $v \in W^{1, \infty}(\mathbb{R}, \mathbb{C})$.

(iv) The maps $\omega \mapsto \lambda_{\omega}$ and $\omega \mapsto \phi_{\omega}$ are $C^{2}$-smooth, with $\lambda_{0}=0$ and $\phi_{0}=\Phi^{\prime}$.

In the present paper we focus on developing a comparison-principle based approach to understand the stability of (2.8), avoiding the spectral techniques that lie at the heart of [24]. Nevertheless, we need to impose one of the spectral condition stated in [24]. In particular, the next assumption ensures that the curve $\omega \rightarrow \lambda_{\omega}$ touches the origin in a quadratic tangency that opens up on the left side of the imaginary axis.

$(H S)_{\zeta_{*}}$ Recalling the curves $\omega \mapsto\left(\lambda_{\omega}, \phi_{\omega}\right)$ defined in Proposition 2.1, we have the Melnikov identity

$$
\left[\frac{d^{2}}{d \omega^{2}} \lambda_{\omega}\right]_{\omega=0}<0
$$

We emphasize here that $(H S)_{\zeta_{*}}$ is guaranteed to be satisfied whenever $\zeta_{*} \approx \frac{k \pi}{4}$ and $c_{\frac{k \pi}{4}}>0$ for some $k \in \mathbb{Z}$. In addition, we have numerical evidence to suggest that $(H S)_{\zeta_{*}}$ is satisfied generically; see $[24, \S 6]$ for a detailed discussion.

We are now ready to state our first main result, which will be proved in $\S 5$. Compared to the small-perturbation stability result in 24], we note that here the initial deviation from the wave can be very large in compact regions. On the other hand, in [24] the perturbations are not required to decay in the direction parallel to the wave propagation as they are here. 
Theorem 2.2. Consider the unobstructed LDE (2.1) and suppose that (Hg) is satisfied. Pick a direction $\zeta_{*} \in \mathbb{R}$ for which $\tan \zeta_{*} \in \mathbb{Q}$ and suppose that the requirements stated in (H$\left.\Phi\right)$ all hold, but only for the angle $\zeta_{*}$. In addition, suppose that $(H S)_{\zeta_{*}}$ is satisfied.

Consider any $C^{1}$-smooth function $U:[0, \infty) \rightarrow \ell^{\infty}\left(\mathbb{Z}^{2} ; \mathbb{R}\right)$ that satisfies the LDE (2.1) for all $t \geq 0$. Suppose furthermore that we have the spatial limit

$$
\left|U_{i j}(0)-\Phi_{\zeta_{*}}\left(i \cos \zeta_{*}+j \sin \zeta_{*}\right)\right| \rightarrow 0, \quad|i|+|j| \rightarrow \infty
$$

Then we have the uniform convergence

$$
\sup _{(i, j) \in \mathbb{Z}^{2}}\left|U_{i j}(t)-\Phi_{\zeta_{*}}\left(i \cos \zeta_{*}+j \sin \zeta_{*}+c_{\zeta_{*}} t\right)\right| \rightarrow 0, \quad t \rightarrow \infty .
$$

\subsection{Obstructed Lattice}

In order to formalize the concept of removing grid points from a lattice, we start by introducing some notation. In particular, for any $(i, j) \in \mathbb{Z}^{2}$ we write

$$
\mathcal{N}_{\mathbb{Z}^{2}}(i, j)=\{(i+1, j),(i, j+1),(i-1, j),(i, j-1)\} \subset \mathbb{Z}^{2}
$$

to denote the set of nearest neighbours for the grid point $(i, j)$. Obviously, we can now restate the definition $(2.2)$ as

$$
\left[\Delta^{+} u\right]_{i j}=\sum_{\left(i^{\prime}, j^{\prime}\right) \in \mathcal{N}_{\mathbb{Z}^{2}}(i, j)}\left[u_{i^{\prime}, j^{\prime}}-u_{i j}\right]
$$

Consider now a bounded set $K_{\text {obs }} \subset \mathbb{Z}^{2}$, which should be interpreted to be missing from the lattice. We write

$$
\Lambda=\mathbb{Z}^{2} \backslash K_{\text {obs }}
$$

to refer to the remaining grid points. In addition, for any point $(i, j) \in \Lambda$, we write

$$
\mathcal{N}_{\Lambda}(i, j)=\mathcal{N}_{\mathbb{Z}^{2}}(i, j) \cap \Lambda,
$$

which represents the set of traditional nearest neighbours of $(i, j)$ that are not contained in $K_{\text {obs }}$. We use the suggestive notation

$$
\partial \Lambda=\left\{(i, j) \in \Lambda: \mathcal{N}_{\mathbb{Z}^{2}}(i, j) \neq \mathcal{N}_{\Lambda}(i, j)\right\}
$$

to denote the set of points in the lattice that are nearest neighbour to a site in the obstacle $K_{\text {obs }}$.

Let us now consider a sequence $v \in \ell^{\infty}(\Lambda, \mathbb{R})$ and introduce the punctured discrete Laplacian that acts as

$$
\left[\Delta_{\Lambda}^{+} v\right]_{i j}=\sum_{\left(i^{\prime}, j^{\prime}\right) \in \mathcal{N}_{\Lambda}(i, j)}\left[v_{i^{\prime} j^{\prime}}-v_{i j}\right]
$$

for $(i, j) \in \Lambda$. Obviously, we have

$$
\left[\Delta_{\Lambda}^{+} v\right]_{i j}=\left[\Delta^{+} v\right]_{i j}, \quad(i, j) \in \Lambda \backslash \partial \Lambda .
$$

Our main goal in this paper is to study the obstructed LDE

$$
\dot{u}_{i j}(t)=\left[\Delta_{\Lambda}^{+} u(t)\right]_{i j}+g(u(t)), \quad(i, j) \in \Lambda .
$$

As a consequence of our choice for $\Delta_{\Lambda}^{+}$, one can interpret this LDE as the analogue of a reactiondiffusion PDE posed on an exterior domain under Neumann boundary conditions.

Besides the conditions imposed above on the homogeneous LDE (2.1), we need to impose the following two conditions on the obstacle $K_{\mathrm{obs}}$. 
(HK1) The obstacle set $K_{\text {obs }}$ is bounded and $\Lambda=\mathbb{Z}^{2} \backslash K_{\text {obs }}$ is connected, in the sense that for every $(i, j) \in \Lambda$ and $\left(i^{\prime}, j^{\prime}\right) \in \Lambda$, there exists an integer $N \geq 0$ and a sequence $\left\{\left(i_{k}, j_{k}\right)\right\}_{k=0}^{N} \subset \Lambda$ with

$$
\left(i_{0}, j_{0}\right)=\left(i^{\prime}, j^{\prime}\right), \quad\left(i_{N}, j_{N}\right)=(i, j),
$$

so that for every $1 \leq k \leq N$ we have

$$
\left(i_{k}, j_{k}\right) \in \mathcal{N}_{\Lambda}\left(i_{k-1}, j_{k-1}\right)
$$

(HK2) The obstacle $K_{\text {obs }}$ is directionally convex, in the sense that there exists a line $\ell \subset \mathbb{R}^{2}$ so that the following holds true. For any $(i, j) \in \partial \Lambda$ and $\left(i^{\prime}, j^{\prime}\right) \in K_{\text {obs }}$ that are related via $\left(i^{\prime}, j^{\prime}\right) \in \mathcal{N}_{\mathbb{Z}^{2}}(i, j)$, we have

$$
d\left(\left(i^{\prime}, j^{\prime}\right), \ell\right) \leq d((i, j), \ell)
$$

in which $d((x, y), \ell) \geq 0$ denotes the distance between a point $(x, y) \in \mathbb{R}^{2}$ and the line $\ell \subset \mathbb{R}^{2}$.

These conditions can be seen as the discrete analogues of the restrictions imposed in [9]. However, we remark here that we exclude the star-shaped obstacles that are allowed in [9]. In any case, it is easy to see that any obstacle that consists of a single point automatically satisfies both (HK1) and (HK2).

We are now ready to state our second main result, which is the discrete analogue of 9 , Thm. 1.3]. It basically states that there is an entire solution to (2.31) that looks like a travelling planar wave travelling towards the obstacle for $t \ll-1$, gets scattered by the obstacle at $t=O(1)$ and gradually recovers its shape as the wavefront moves away from the obstacle for $t \gg+1$.

Theorem 2.3. Consider the obstructed $L D E$ (2.31) and pick a direction $\zeta_{*} \in \mathbb{R}$ for which $\tan \zeta_{*} \in \mathbb{Q}$.

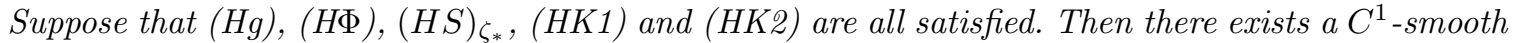
function $U: \mathbb{R} \rightarrow \ell^{\infty}(\Lambda, \mathbb{R})$ that satisfies the obstructed $L D E$ (2.31) for all $t \in \mathbb{R}$, admits the inequalities

$$
0<U_{i j}(t)<1, \quad \dot{U}_{i j}(t)>0
$$

for all $t \in \mathbb{R}$ and $(i, j) \in \Lambda$ and enjoys the temporal limits

$$
\sup _{(i, j) \in \Lambda}\left|U_{i j}(t)-\Phi_{\zeta_{*}}\left(i \cos \zeta_{*}+j \sin \zeta_{*}+c_{\zeta_{*}} t\right)\right| \rightarrow 0, \quad t \rightarrow \pm \infty
$$

together with the spatial limit

$$
\sup _{t \in \mathbb{R}}\left|U_{i j}(t)-\Phi_{\zeta_{*}}\left(i \cos \zeta_{*}+j \sin \zeta_{*}+c_{\zeta_{*}} t\right)\right| \rightarrow 0, \quad|i|+|j| \rightarrow \infty .
$$

In addition, if $V: \mathbb{R} \rightarrow \ell^{\infty}(\Lambda ; \mathbb{R})$ is another $C^{1}$-smooth function that satisfies the obstructed LDE (2.31) for all $t \in \mathbb{R}$ with

$$
\sup _{(i, j) \in \Lambda}\left|V_{i j}(t)-\Phi_{\zeta_{*}}\left(i \cos \zeta_{*}+j \sin \zeta_{*}+c_{\zeta_{*}} t\right)\right| \rightarrow 0, \quad t \rightarrow-\infty,
$$

then we have $U=V$.

\section{Preliminaries}

In this section we consider an obstacle $K_{\text {obs }} \subset \mathbb{Z}^{2}$, which at times will be taken to be empty, and study the LDE

$$
\dot{u}_{i j}(t)=\left[\Delta_{\Lambda}^{+} u(t)\right]_{i j}+g\left(u_{i j}(t)\right), \quad(i, j) \in \Lambda,
$$

in which $\Lambda=\mathbb{Z}^{2} \backslash K_{\text {obs. }}$. We formulate a comparison principle for (3.1) and focus on the asymptotic behaviour of travelling wave solutions to (3.1) with $K_{\mathrm{obs}}=\emptyset$. We are specifically interested in the 
dependence of these waves on the direction of propagation and the specific form of the nonlinearity, which we will often need to distort.

For the purposes of this section, we need to relax the smoothness requirements present in $(\mathrm{Hg})$. In particular, we introduce the following condition on the nonlinearity $g$.

(hg) $)_{\S 3}$ The nonlinearity $g$ is $C^{1}$-smooth, while the map $u \mapsto g^{\prime}(u)$ is locally Lipschitz continuous. In addition, there exists a constant $0<a<1$ such that we have

$$
g(0)=g(a)=g(1)=0, \quad g^{\prime}(0)<0, \quad g^{\prime}(1)<0,
$$

together with

$$
g(u)<0 \text { for } u \in(0, a) \cup(1, \infty), \quad g(u)>0 \text { for } u \in(-\infty,-1) \cup(a, 1) .
$$

We start by formulating a weak and strong version of a comparison principle for (3.1). The weak version does not need the obstacle to be finite or connected as required by (HK1).

Proposition 3.1. Pick any subset $K_{\mathrm{obs}} \subset \mathbb{Z}^{2}$, consider the LDE (3.1) and suppose that $(\mathrm{hg})_{\S 3}$ is satisfied. Consider a pair of functions $u, v \in C^{1}\left([0, \infty), \ell^{\infty}(\Lambda, \mathbb{R})\right)$ that satisfy the uniform bounds

$$
-1 \leq u_{i j}(t) \leq 2, \quad-1 \leq v_{i j}(t) \leq 2, \quad(i, j) \in \Lambda, \quad t \geq 0,
$$

together with the initial inequalities

$$
u_{i j}(0) \geq v_{i j}(0), \quad(i, j) \in \Lambda .
$$

Suppose furthermore that for any $t \geq 0$ and all $(i, j) \in \Lambda$, at least one of the following two properties is satisfied.

(a) We have the differential inequalities

$$
\dot{u}_{i j}(t) \geq\left[\Delta_{\Lambda}^{+} u(t)\right]_{i j}+g\left(u_{i j}(t)\right), \quad \dot{v}_{i j}(t) \leq\left[\Delta_{\Lambda}^{+} v(t)\right]_{i j}+g\left(v_{i j}(t)\right) .
$$

(b) We have the inequality $u_{i j}(t) \geq v_{i j}(t)$.

Then we in fact have $u_{i j}(t) \geq v_{i j}(t)$ for all $(i, j) \in \Lambda$ and $t \geq 0$.

Corollary 3.2. Consider the setting of Proposition 3.1. Suppose that the obstacle $K_{\mathrm{obs}}$ satisfies (HK1) and that there exists $\left(i_{0}, j_{0}\right) \in \Lambda$ for which $u_{i_{0} j_{0}}(0)>v_{i_{0} j_{0}}(0)$. Then we have the strict inequality

$$
u_{i j}(t)>v_{i j}(t), \quad(i, j) \in \Lambda, \quad t>0 .
$$

Proof. Suppose that for some $t_{*}>0$ and $\left(i_{*}, j_{*}\right) \in \Lambda$, we have $u_{i_{*} j_{*}}\left(t_{*}\right)=v_{i_{*} j_{*}}\left(t_{*}\right)$. Since $u_{i j}(t) \geq$ $v_{i j}(t)$ for all $t \geq 0$ and $(i, j) \in \Lambda$, we must have $\dot{u}_{i_{*} j_{*}}\left(t_{*}\right)=\dot{v}_{i_{*} j_{*}}\left(t_{*}\right)$. In particular, this implies

$$
\left[\Delta_{\Lambda}^{+} u\left(t_{*}\right)\right]_{i_{*} j_{*}}=\left[\Delta_{\Lambda}^{+} v\left(t_{*}\right)\right]_{i_{*} j_{*}}
$$

which in turn shows that we must have $u_{i j}\left(t_{*}\right)=v_{i j}\left(t_{*}\right)$ for all $(i, j) \in \mathcal{N}_{\Lambda}\left(i_{*}, j_{*}\right)$. Exploiting the connectedness of $\Lambda$, this argument can be repeated to show that $u\left(t_{*}\right)=v\left(t_{*}\right)$, which contradicts the uniqueness of solutions to (3.1) in backward time.

We now turn our attention to travelling wave solutions of the unobstructed system (3.1) with $\Lambda=\mathbb{Z}^{2}$. To this end, we pick an arbitrary pair $\left(\sigma_{h}, \sigma_{v}\right) \in \mathbb{R}^{2}$, assuming only that $\sigma_{h}^{2}+\sigma_{v}^{2} \neq 0$. Inserting the travelling wave Ansatz

$$
u_{i j}(t)=\Phi\left(i \sigma_{h}+j \sigma_{v}+c t\right)
$$

into the homogeneous LDE (3.1), we arrive at the travelling wave MFDE

$$
c \Phi^{\prime}(\xi)=\Phi\left(\xi+\sigma_{h}\right)+\Phi\left(\xi+\sigma_{v}\right)+\Phi\left(\xi-\sigma_{h}\right)+\Phi\left(\xi-\sigma_{v}\right)-4 \Phi(\xi)+g(\Phi(\xi)) .
$$

The following assumption relating to the existence of solutions to (3.10) with non-zero wave speed is used frequently throughout this entire paper. 
$(\mathrm{h} \Phi)_{\S 3}$ The travelling wave system (3.10) admits a solution $(c, \Phi)$ for some $c \neq 0$ and bounded function $\Phi \in C^{2}(\mathbb{R}, \mathbb{R})$ that satisfies the limits

$$
\lim _{\xi \rightarrow-\infty} \Phi(\xi)=0, \quad \lim _{\xi \rightarrow+\infty} \Phi(\xi)=1 .
$$

We note that the $C^{1}$-smoothness of the nonlinearity $g$ prescribed by $(\mathrm{hg})_{\S 3}$ ensures that the $C^{2}$ continuity mentioned in $(\mathrm{h} \Phi)_{\S 3}$ is automatic upon assuming that $\Phi$ is merely continuous.

An important role is played by the asymptotic rates at which the wave profile $\Phi$ approaches its limiting values. To study these rates, we introduce the limiting spatial characteristic functions

$$
\begin{aligned}
& \Delta^{-}(z)=c z-\left(2 \cosh \left(\sigma_{h} z\right)+2 \cosh \left(\sigma_{v} z\right)-4\right)-g^{\prime}(0), \\
& \Delta^{+}(z)=c z-\left(2 \cosh \left(\sigma_{h} z\right)+2 \cosh \left(\sigma_{v} z\right)-4\right)-g^{\prime}(1) .
\end{aligned}
$$

It is well known that the real roots of the equations $\Delta^{ \pm}(z)=0$ are directly related to the asymptotic convergence rates discussed above.

Lemma 3.3. Consider the characteristic equations (3.12) and suppose that $(\mathrm{hg})_{\S 3}$ and $(h \Phi)_{\S 3}$ both hold. Then there exist constants $\eta_{\Phi}^{ \pm}>0$ that satisfy the identities

$$
\begin{aligned}
& c \eta_{\Phi}^{-}=2 \cosh \left(\sigma_{h} \eta_{\Phi}^{-}\right)+2 \cosh \left(\sigma_{v} \eta_{\Phi}^{-}\right)-4+g^{\prime}(0), \\
& -c \eta_{\Phi}^{+}=2 \cosh \left(\sigma_{h} \eta_{\Phi}^{+}\right)+2 \cosh \left(\sigma_{v} \eta_{\Phi}^{+}\right)-4+g^{\prime}(1),
\end{aligned}
$$

which implies that

$$
\Delta^{+}\left(-\eta_{\Phi}^{+}\right)=\Delta^{-}\left(\eta_{\Phi}^{-}\right)=0 .
$$

In addition, if either $\Delta^{+}(-\eta)=0$ or $\Delta^{-}(\eta)=0$ holds for any $\eta \geq 0$, then we must have $\eta=\eta_{\Phi}^{+}$or $\eta=\eta_{\Phi}^{-}$respectively.

Finally, if the inequalities

$$
c>0, \quad \eta_{\Phi}^{-} \leq \eta_{\Phi}^{+}
$$

are both satisfied, then the inequality

$$
g^{\prime}(0)>g^{\prime}(1)
$$

must also hold.

Proof. The statements follow directly from the observation that $\left[\Delta^{ \pm}\right]^{\prime \prime}(z)<0$ for all $z \in \mathbb{R}$, together with the limits $\lim _{z \rightarrow \pm \infty} \Delta^{ \pm}(z)=-\infty$ and the inequalities $\Delta^{ \pm}(0)>0$.

Proposition 3.4. Consider the travelling wave MFDE (3.10), assume that $(h g)_{\S 3}$ and $(h \Phi)_{\S 3}$ both hold and recall the spatial exponents $\eta_{\Phi}^{ \pm}$defined in Lemma 3.3. Then there exist constants $K_{\Phi}>1$, $\kappa_{\Phi}>0$ and $C_{\Phi}^{ \pm}>0$ such that for every $\xi \leq 0$ we have

$$
\begin{aligned}
& \mid \Phi(\xi)-C_{\Phi}^{-} e^{-\eta_{\Phi}^{-}|\xi|} \leq K_{\Phi} e^{-\left(\eta_{\Phi}^{-}+\kappa_{\Phi}\right)|\xi|} \\
& \left|\Phi^{\prime}(\xi)-\eta_{\Phi}^{-} C_{\Phi}^{-} e^{-\eta_{\Phi}^{-}|\xi|}\right| \leq K_{\Phi} e^{-\left(\eta_{\Phi}^{-}+\kappa_{\Phi}\right)|\xi|} \\
& \left|\Phi^{\prime \prime}(\xi)-\left[\eta_{\Phi}^{-}\right]^{2} C_{\Phi}^{-} e^{-\eta_{\Phi}^{-}|\xi|}\right| \leq K_{\Phi} e^{-\left(\eta_{\Phi}^{-}+\kappa_{\Phi}\right)|\xi|}
\end{aligned}
$$

while for every $\xi \geq 0$ we have

$$
\begin{aligned}
& \left|(1-\Phi(\xi))-C_{\Phi}^{+} e^{-\eta_{\Phi}^{+}|\xi|}\right| \leq K_{\Phi} e^{-\left(\eta_{\Phi}^{+}+\kappa_{\Phi}\right)|\xi|} \\
& \left|\Phi^{\prime}(\xi)-\eta_{\Phi}^{+} C_{\Phi}^{+} e^{-\eta_{\Phi}^{+}|\xi|}\right| \leq K_{\Phi} e^{-\left(\eta_{\Phi}^{+}+\kappa_{\Phi}\right)|\xi|} \\
& \left|\Phi^{\prime \prime}(\xi)+\left[\eta_{\Phi}^{+}\right]^{2} C_{\Phi}^{+} e^{-\eta_{\Phi}^{+}|\xi|}\right| \leq K_{\Phi} e^{-\left(\eta_{\Phi}^{+}+\kappa_{\Phi}\right)|\xi|} .
\end{aligned}
$$


Proof. These bounds follow directly from [35, Thm. 2.2].

Corollary 3.5. Consider the setting of Proposition 3.4. Then there exist constants

$$
0<\alpha_{\text {low }}^{ \pm}<\alpha_{\text {up }}^{ \pm}, \quad 0<\beta_{\text {low }}^{ \pm}<\beta_{\text {up }}^{ \pm}
$$

such that for every $\xi \leq 0$ we have

$$
\begin{aligned}
& \beta_{\text {low }}^{-} e^{-\eta_{\Phi}^{-}|\xi|} \leq \Phi(\xi) \leq \beta_{\text {up }}^{-} e^{-\eta_{\Phi}^{-}|\xi|} \\
& \alpha_{\text {low }}^{-} e^{-\eta_{\Phi}^{-}|\xi|} \leq \Phi^{\prime}(\xi) \leq \alpha_{\text {up }}^{-} e^{-\eta_{\Phi}^{-}|\xi|},
\end{aligned}
$$

while for every $\xi \geq 0$ we have

$$
\begin{aligned}
& \beta_{\text {low }}^{+} e^{-\eta_{\Phi}^{+}|\xi|} \leq 1-\Phi(\xi) \leq \beta_{\text {up }}^{+} e^{-\eta_{\Phi}^{+}|\xi|} \\
& \alpha_{\text {low }}^{+} e^{-\eta_{\Phi}^{+}|\xi|} \leq \Phi^{\prime}(\xi) \leq \alpha_{\text {up }}^{+} e^{-\eta_{\Phi}^{+}|\xi|} .
\end{aligned}
$$

Proof. These identities follow directly from Proposition 3.4, upon exploiting the fact that the inequalities $0<\Phi(\xi)<1$ and $\Phi^{\prime}(\xi)>0$ hold for all $\xi \in \mathbb{R}$.

Corollary 3.6. Consider the travelling wave MFDE (3.10) and assume that $(\mathrm{hg})_{\S 3}$ and $(h \Phi)_{\S 3}$ both hold. Then for every $M>1$, there exists a constant $K_{\text {shift }}=K_{\text {shift }}(M)$ so that

$$
\left|\frac{\Phi^{\prime \prime}(\zeta)}{\Phi^{\prime}(\xi)}\right|+\left|\frac{\Phi^{\prime}(\zeta)}{\Phi^{\prime}(\xi)}\right| \leq K_{\text {shift }}
$$

holds for every pair $(\zeta, \xi) \in \mathbb{R}^{2}$ for which $|\zeta-\xi| \leq M$.

Proof. This follows from the fact that $\Phi^{\prime}(\xi)>0$ for all $\xi \in \mathbb{R}$ together with the asymptotic bounds stated in Proposition 3.4 .

Our final main result in this section roughly states that the properties described above for the wave $(c, \Phi)$ vary continuously upon changing the direction $\left(\sigma_{h}, \sigma_{v}\right)$ and perturbing the nonlinearity $g$. We note that the results in [35, Thm. 2.1] cover neither variations in the direction of propagation nor smoothness properties of asymptotic expansions, so we take the opportunity here to discuss these issues in depth.

In order to state the result, we introduce for any $\eta \in \mathbb{R}$ and any interval $\mathcal{I} \subset \mathbb{R}$ the exponentially weighted function spaces

$$
\begin{aligned}
B C_{\eta}(\mathcal{I}, \mathbb{R}) & =\left\{p \in C(\mathcal{I}, \mathbb{R})\left|\sup _{\xi \in \mathcal{I}} e^{-\eta|\xi|}\right| p(\xi) \mid<\infty\right\} \\
B C_{\eta}^{1}(\mathcal{I}, \mathbb{R}) & =\left\{p \in C^{1}(\mathcal{I}, \mathbb{R}) \mid \sup _{\xi \in \mathcal{I}} e^{-\eta|\xi|}\left[|p(\xi)|+\left|p^{\prime}(\xi)\right|\right]<\infty\right\} .
\end{aligned}
$$

Proposition 3.7. Consider the travelling wave MFDE (3.10) and suppose that $(h g)_{\S 3}$ and $(h \Phi)_{\S 3}$ with $c>0$ are both satisfied. Fix $\delta_{p}>0$ sufficiently small and consider the set

$$
\Omega=\left\{\left(\delta, \sigma_{h}^{\prime}, \sigma_{v}^{\prime}\right): 0 \leq \delta<\delta_{p} \text { and }\left|\sigma_{h}^{\prime}-\sigma_{h}\right|+\left|\sigma_{v}^{\prime}-\sigma_{v}\right|<\delta_{p}\right\} .
$$

Then for any $p=\left(\delta, \sigma_{h}^{\prime}, \sigma_{v}^{\prime}\right) \in \Omega$, there exist $C^{2}$-smooth functions $\Phi_{p}^{ \pm}: \mathbb{R} \rightarrow \mathbb{R}$ and constants $c_{p}^{ \pm}>0$ such that the following properties hold.

(i) For any $p=\left(\delta, \sigma_{h}^{\prime}, \sigma_{v}^{\prime}\right) \in \Omega$, the MFDEs

$$
\begin{gathered}
c_{p}^{ \pm}\left[\Phi_{p}^{ \pm}\right]^{\prime}(\xi)=\Phi_{p}^{ \pm}\left(\xi+\sigma_{h}^{\prime}\right)+\Phi_{p}^{ \pm}\left(\xi+\sigma_{v}^{\prime}\right)+\Phi_{p}^{ \pm}\left(\xi-\sigma_{h}^{\prime}\right)+\Phi_{p}^{ \pm}\left(\xi-\sigma_{v}^{\prime}\right)-4 \Phi_{p}^{ \pm}(\xi) \\
+g_{\delta}^{ \pm}\left(\Phi_{p}^{ \pm}(\xi)\right)
\end{gathered}
$$

are satisfied for all $\xi \in \mathbb{R}$. Here the maps $u \rightarrow g_{\delta}^{ \pm}(u \mp \delta)$ satisfy $(h g)_{\S 3}$, while for any $u \in \mathbb{R}$ we have $g_{0}^{ \pm}(u)=g(u)$ and $g_{\delta}^{-}(u) \leq g(u) \leq g_{\delta}^{+}(u)$. 
(ii) For any $p=\left(\delta, \sigma_{h}^{\prime}, \sigma_{v}^{\prime}\right) \in \Omega$, we have $\left[\Phi_{p}^{ \pm}\right]^{\prime}(\xi)>0$, together with the four limits

$$
\begin{array}{ll}
\lim _{\xi \rightarrow-\infty} \Phi_{p}^{-}(\xi)=-\delta, & \lim _{\xi \rightarrow+\infty} \Phi_{p}^{-}(\xi)=1-\delta, \\
\lim _{\xi \rightarrow-\infty} \Phi_{p}^{+}(\xi)=+\delta, & \lim _{\xi \rightarrow+\infty} \Phi_{p}^{+}(\xi)=1+\delta .
\end{array}
$$

(iii) For any $p=\left(\delta, \sigma_{h}^{\prime}, \sigma_{v}^{\prime}\right) \in \Omega$, the functions $W_{p}^{ \pm}: \mathbb{R} \rightarrow \ell^{\infty}\left(\mathbb{Z}^{2}, \mathbb{R}\right)$ defined by

$$
\left[W_{p}^{ \pm}\right]_{i j}(t)=\Phi_{p}^{ \pm}\left(\sigma_{h}^{\prime} i+\sigma_{v}^{\prime} j+c_{p}^{ \pm} t\right)
$$

satisfy the differential inequalities

$$
\mathcal{J}_{i j}^{-}(t) \leq 0 \leq \mathcal{J}_{i j}^{+}(t)
$$

in which

$$
\mathcal{J}_{i j}^{ \pm}(t)=\left[\dot{W}_{p}^{ \pm}\right]_{i j}(t)-\left[\Delta^{+} W_{p}^{ \pm}(t)\right]_{i j}-g\left(\left[W_{p}^{ \pm}\right]_{i j}(t)\right) .
$$

In addition, if $\delta=0$, then we have $W_{p}^{-}=W_{p}^{+}$and the inequalities in (3.28) are equalities.

(iv) Upon writing $\eta_{\Phi}=\min \left\{\eta_{\Phi}^{+}, \eta_{\Phi}^{-}\right\}$, the maps

$$
\left(\nu, \sigma_{h}^{\prime}, \sigma_{v}^{\prime}\right) \mapsto\left\{\begin{array}{l}
c_{\nu^{2}, \sigma_{h}^{\prime}, \sigma_{v}^{\prime}}^{ \pm} \in \mathbb{R} \\
\Phi_{\nu^{2}, \sigma_{h}^{\prime}, \sigma_{v}^{\prime}}+\nu^{2}-\Phi \in B C_{-\frac{1}{2} \eta_{\Phi}}(\mathbb{R}, \mathbb{R}) \\
\Phi_{\nu^{2}, \sigma_{h}^{\prime}, \sigma_{v}^{\prime}}^{+}-\nu^{2}-\Phi \in B C_{-\frac{1}{2} \eta_{\Phi}}(\mathbb{R}, \mathbb{R})
\end{array}\right.
$$

are $C^{1}$-smooth, with

$$
c_{0, \sigma_{h}, \sigma_{v}}^{ \pm}=c, \quad \Phi_{0, \sigma_{h}, \sigma_{v}}^{ \pm}=\Phi .
$$

(v) For any $p=\left(\delta, \sigma_{h}^{\prime}, \sigma_{v}^{\prime}\right) \in \Omega$, the function $\Phi_{p}^{-}+\delta$ satisfies the asymptotic estimates (3.17)(3.18) with constants $K_{\Phi}>1$ and $\kappa_{\Phi}>0$ that are independent of $p$, constants $\eta_{\Phi}^{ \pm}>0$ that depend $C^{1}$-smoothly on $\left(\sigma_{h}^{\prime}, \sigma_{v}^{\prime}\right)$ but are independent of $\delta$, together with constants $C_{\Phi}^{ \pm}$that depend $C^{1}$-smoothly on $\left(\sqrt{\delta}, \sigma_{h}^{\prime}, \sigma_{v}^{\prime}\right)$. A similar statement holds for the functions $\Phi_{p}^{+}-\delta$.

In the remainder of this section we provide the missing proofs for the results stated above. We start by establishing the weak comparison principle, closely following the arguments in 29, Prop. 4.1], which in turn are based on [13].

Proof of Proposition 3.1. Upon writing $w_{i j}(t)=u_{i j}(t)-v_{i j}(t)$ together with

$$
\mathcal{I}_{i j}(t)=\int_{0}^{1} g^{\prime}\left(v_{i j}(t)+\vartheta w_{i j}(t)\right) d \vartheta
$$

the estimate

$$
\begin{aligned}
\dot{w}_{i j}(t) & \geq\left[\Delta_{\Lambda}^{+} w(t)\right]_{i j}+g\left(u_{i j}(t)\right)-g\left(v_{i j}(t)\right) \\
& =\left[\Delta_{\Lambda}^{+} w(t)\right]_{i j}+\mathcal{I}_{i j}(t) w_{i j}(t)
\end{aligned}
$$

holds for all $(i, j) \in \Lambda$ and $t \geq 0$ for which condition (a) is satisfied.

In order to show that $w_{i j}(t) \geq 0$ for all $t \geq 0$ and $(i, j) \in \Lambda$, let us assume to the contrary that this is false. In particular, suppose that there exist $t_{*}>0,\left(i_{*}, j_{*}\right) \in \Lambda$ for which $w_{i_{*}, j_{*}}\left(t_{*}\right)=-\vartheta<0$. Picking $\epsilon>0$ and $K^{\prime}>0$ in such a way that $\vartheta=\epsilon e^{2 K^{\prime} t_{*}}$, we can now define

$$
T^{\prime}:=\sup \left\{t \geq 0 \mid w_{i j}(t)>-\epsilon e^{2 K^{\prime} t} \text { for all }(i, j) \in \Lambda\right\} .
$$

The $C^{1}$-smoothness of $w$ guarantees that $0<T^{\prime} \leq t_{*}$. In addition, we have

$$
\inf _{(i, j) \in \Lambda} w_{i j}\left(T^{\prime}\right)=-\epsilon e^{2 K^{\prime} T^{\prime}}
$$


since otherwise the smoothness of $w$ as map into $\ell^{\infty}(\Lambda, \mathbb{R})$ would allow the constant $T^{\prime}$ to be increased. Without loss of generality we may therefore assume that $(0,0) \in \Lambda$ and $w_{0,0}\left(T^{\prime}\right)<$ $-\frac{7}{8} \epsilon e^{2 K^{\prime} T^{\prime}}$.

Consider now the function

$$
w_{i j}^{-}(t ; \beta)=-\epsilon\left(\frac{3}{4}+\beta z_{i j}\right) e^{2 K^{\prime} t},
$$

in which $\beta>0$ is a parameter and $z \in \ell^{\infty}(\Lambda ; \mathbb{R})$ has $z_{0,0}=1, \lim _{|i|+|j| \rightarrow \infty} z_{i j}=3,1 \leq z \leq 3$ and $\left|\Delta_{\Lambda}^{+} z\right| \leq 1$. Write $\beta_{*} \in\left(\frac{1}{8}, \frac{1}{4}\right]$ for the minimal value of $\beta$ for which $w_{i j}(t) \geq w_{i j}^{-}(t ; \beta)$ holds for all $(i, j, t) \in \Lambda \times\left[0, T^{\prime}\right]$. Since

$$
\lim _{|i|+|j| \rightarrow \infty} w_{i j}^{-}\left(t ; \beta_{*}\right)=-\epsilon\left[\frac{3}{4}+3 \beta_{*}\right] e^{2 K^{\prime} t}<-\frac{9}{8} \epsilon e^{2 K^{\prime} t},
$$

there exist $\left(i_{0}, j_{0}\right) \in \Lambda$ and $0<t_{0} \leq T^{\prime}$ such that $w_{i_{0}, j_{0}}\left(t_{0}\right)=w_{i_{0}, j_{0}}^{-}\left(t_{0} ; \beta_{*}\right)$. The definition of $\beta_{*}$ now implies that

$$
\dot{w}_{i_{0}, j_{0}}\left(t_{0}\right) \leq \dot{w}_{i_{0}, j_{0}}^{-}\left(t_{0} ; \beta_{*}\right)
$$

In addition, by positivity of the off-diagonal coefficients in $\Delta_{\Lambda}^{+}$, we have

$$
\left[\Delta_{\Lambda}^{+} w\left(t_{0}\right)\right]_{i_{0}, j_{0}} \geq\left[\Delta_{\Lambda}^{+} w^{-}\left(t_{0} ; \beta_{*}\right)\right]_{i_{0}, j_{0}} .
$$

Using the fact that $w_{i_{0}, j_{0}}\left(t_{0}\right)<0$, we see that (a) and hence (3.33) is satisfied, which leads to the estimate

$$
\begin{aligned}
-\frac{7}{4} \epsilon K^{\prime} e^{2 K^{\prime} t_{0}} & \geq \dot{w}_{i_{0}, j_{0}}^{-}\left(t_{0}\right) \geq \dot{w}_{i_{0}, j_{0}}\left(t_{0}\right) \\
& \geq\left[\Delta_{\Lambda}^{+} w\left(t_{0}\right)\right]_{i_{0}, j_{0}}+\mathcal{I}_{i_{0}, j_{0}}\left(t_{0}\right) w_{i_{0}, j_{0}}\left(t_{0}\right) \\
& \geq\left[\Delta_{\Lambda}^{+} w^{-}\left(t_{0} ; \beta_{*}\right)\right]_{i_{0}, j_{0}}+\mathcal{I}_{i_{0}, j_{0}}\left(t_{0}\right) w_{i_{0}, j_{0}}^{-}\left(t_{0} ; \beta_{*}\right) .
\end{aligned}
$$

In particular, we obtain the bound

$$
-\frac{7}{4} \epsilon K^{\prime} e^{2 K^{\prime} t_{0}} \geq-3 \epsilon\left[1+M^{\prime}\right] e^{2 K^{\prime} t_{0}}
$$

in which

$$
M^{\prime}=\sup _{-1 \leq u \leq 2}\left|g^{\prime}(u)\right| .
$$

This leads to a contradiction upon choosing $K^{\prime} \gg 1$ to be sufficiently large, showing that indeed $w_{i j}(t) \geq 0$ for all $(i, j) \in \Lambda$ and $t \geq 0$.

We now turn our attention to the results stated in Proposition 3.7. Our first concern is to construct the distorted nonlinearities mentioned in item (i).

Lemma 3.8. There exist two $C^{1}$-smooth functions

$$
\tau^{ \pm}: \mathbb{R} \times\left(-\infty, \frac{1}{12}\right] \rightarrow \mathbb{R}
$$

that satisfy the following properties.

(i) For any $\nu \in\left(0, \frac{1}{12}\right)$ and any $u \in[\nu, 1-\nu]$, we have $\tau^{ \pm}(u, \nu)=u$.

(ii) For any $\nu \in\left(0, \frac{1}{12}\right)$ and any $u \in\left(-\infty,-\nu^{2}\right) \cup\left(1-\nu^{2}, \infty\right)$, we have

$$
\tau^{+}(u, \nu)=u+\nu^{2},
$$

while for any $\nu \in\left(0, \frac{1}{12}\right)$ and any $u \in\left(-\infty, \nu^{2}\right) \cup\left(1+\nu^{2}, \infty\right)$, we have

$$
\tau^{-}(u, \nu)=u-\nu^{2} .
$$


(iii) For any $\nu \leq 0$, we have $\tau^{ \pm}(s, \nu)=s$ for all $s \in \mathbb{R}$.

(iv) For any $0 \leq \nu \leq \frac{1}{12}$ and $u \in \mathbb{R}$, we have the bound

$$
\frac{1}{2} \leq 1-6 \nu \leq \partial_{u} \tau^{ \pm}(u, \nu) \leq 1
$$

together with

$$
\tau^{-}(u, \nu) \leq u \leq \tau^{+}(u, \nu)
$$

(v) There exists a constant $C_{1}>1$ such that

$$
\left[\partial_{u} \tau^{ \pm}(u, \nu)-\partial_{u} \tau^{ \pm}(v, \nu)\right] \leq C_{1}|u-v|
$$

holds for all $\nu \leq \frac{1}{12}$ and $(u, v) \in \mathbb{R}^{2}$.

Proof. We restrict ourselves to defining a function $\tau^{+}: \mathbb{R}^{2} \rightarrow \mathbb{R}$ that satisfies the stated properties whenever $u \leq \frac{1}{2}$ and $\nu \leq \frac{1}{12}$. To this end, we define the three open sets

$$
\begin{aligned}
& \mathcal{V}_{1}=\{\nu<0\} \cup\{u>\nu\} \subset \mathbb{R}^{2}, \\
& \mathcal{V}_{2}=\left\{\nu>0 \text { and } u<-\nu^{2}\right\} \subset \mathbb{R}^{2}, \\
& \mathcal{V}_{3}=\left\{\nu>0 \text { and }-\nu^{2}<u<\nu\right\} \subset \mathbb{R}^{2}
\end{aligned}
$$

together with the three smooth functions

$$
\begin{array}{rlrl}
\tau_{1}: \mathbb{R}^{2} \rightarrow \mathbb{R} & (u, \nu) & \mapsto u, \\
\tau_{2}: \mathbb{R}^{2} \rightarrow \mathbb{R} & (u, \nu) \mapsto u+\nu^{2}, \\
\tau_{3}: \mathbb{R} \times\{\nu>0\} \rightarrow \mathbb{R} & (u, \nu) \mapsto \int_{-\nu^{2}}^{u}\left[1+6 \nu^{-1}(1+\nu)^{-3}\left(s+\nu^{2}\right)(s-\nu)\right] d s .
\end{array}
$$

Upon writing $\tau^{+}(u, \nu)=\tau_{i}(u, \nu)$ whenever $(u, \nu) \in \mathcal{V}_{i}$, we have constructed a $C^{1}$-smooth function $\tau^{+}: \mathcal{V} \rightarrow \mathbb{R}$, where $\mathcal{V}=\mathcal{V}_{1} \cup \mathcal{V}_{2} \cup \mathcal{V}_{3}$

We now set out to show that $\tau^{+}$can be extended to the boundary $\partial \mathcal{V}$ in a smooth fashion. First of all, notice that

$$
\begin{aligned}
\partial_{u} \tau_{3}(u, \nu) & =1+6 \nu^{-1}(1+\nu)^{-3}\left(u+\nu^{2}\right)(u-\nu) \\
& \geq 1-6 \nu^{-1}(1+\nu)^{-3} \frac{1}{4}\left(\nu+\nu^{2}\right)^{2} \\
& \geq 1-6 \nu \frac{1}{4}(1+\nu)^{2} \\
& \geq 1-6 \nu \\
& \geq \frac{1}{2} .
\end{aligned}
$$

In addition, for any $\nu>0$ we have

$$
\partial_{u} \tau_{3}\left(-\nu^{2}, \nu\right)=\partial_{u} \tau_{3}(\nu, \nu)=1
$$

by construction, while a short computation shows that

$$
\tau_{3}\left(-\nu^{2}, \nu\right)=0, \quad \tau_{3}(\nu, \nu)=\nu .
$$

Differentiating these last two identities with respect to $\nu$, we obtain

$$
-2 \nu \partial_{u} \tau_{3}\left(-\nu^{2}, \nu\right)+\partial_{\nu} \tau_{3}\left(-\nu^{2}, \nu\right)=0, \quad \partial_{u} \tau_{3}(\nu, \nu)+\partial_{\nu} \tau_{3}(\nu, \nu)=1
$$


which shows that for all $\nu>0$ we have

$$
\partial_{\nu} \tau_{3}\left(-\nu^{2}, \nu\right)=2 \nu, \quad \partial_{\nu} \tau_{3}(\nu, \nu)=0 .
$$

This suffices to show that $\tau^{+}$can be extended to a $C^{1}$-smooth function on $\mathbb{R}^{2} \backslash\{0,0\}$.

In order to establish $C^{1}$-smoothness at $(0,0)$, we compute

$$
\tau_{3}(u, \nu)=u+\nu^{2}+2 \nu^{-1}(1+\nu)^{-3} u^{3}+(1+\nu)^{-3}\left[2 \nu^{5}+3(\nu-1)\left(u^{2}-\nu^{4}\right)-6 \nu^{2}\left(u+\nu^{2}\right)\right] .
$$

We define $\tau^{+}(0,0)=0$, which together with

$$
\lim _{\nu \downarrow 0} \frac{1}{\nu} \tau^{+}(0, \nu)=\lim _{\nu \downarrow 0} \frac{1}{\nu} \tau_{3}(0, \nu)=0
$$

allows us to conclude

$$
\partial_{u} \tau^{+}(0,0)=1, \quad \partial_{\nu} \tau^{+}(0,0)=0 .
$$

It hence remains to show that for any sequence $\left\{\left(u_{k}, \nu_{k}\right)\right\} \subset \mathcal{V}_{3}$ with $\left(u_{k}, \nu_{k}\right) \rightarrow(0,0)$ we have $\partial_{u} \tau_{3}\left(u_{k}, \nu_{k}\right) \rightarrow 1$ and $\partial_{\nu} \tau_{3}\left(u_{k}, \nu_{k}\right) \rightarrow 0$. We compute

$$
\begin{aligned}
\partial_{u} \tau_{3}(u, \nu)=1+ & 6 \nu^{-1}(1+\nu)^{-3} u^{2}+(1+\nu)^{-3}\left[6(\nu-1) u-6 \nu^{2}\right] \\
\partial_{\nu} \tau_{3}(u, \nu)=2 \nu- & 2 \nu^{-2}(1+\nu)^{-3} u^{3}-6 \nu^{-1}(1+\nu)^{-4} u^{3} \\
& -3(1+\nu)^{-4}\left[2 \nu^{5}+3(\nu-1)\left(u^{2}-\nu^{4}\right)-6 \nu^{2}\left(u+\nu^{2}\right)\right] \\
& +(1+\nu)^{-3}\left[10 \nu^{4}+3\left(u^{2}-\nu^{4}\right)-12(\nu-1) \nu^{3}-12 \nu\left(u+\nu^{2}\right)-12 \nu^{3}\right] .
\end{aligned}
$$

In view of the fact that $\left|u_{k}\right| \leq \nu_{k} \leq 1$ and $\nu_{k} \downarrow 0$, the desired limits can now be read off.

Finally, the Lipschitz property (v) can be easily verified using (3.59).

Lemma 3.9. Suppose that $(\mathrm{hg})_{\S 3}$ is satisfied. For any sufficiently small $\delta>0$, there exist nonlinearities $g_{\delta}^{ \pm}: \mathbb{R} \rightarrow \mathbb{R}$ such that the following properties are satisfied.

(i) For all $u \in \mathbb{R}$ we have the inequalities

$$
g_{\delta}^{-}(u) \leq g(u) \leq g_{\delta}^{+}(u) .
$$

(ii) Recalling the constant a appearing in $(\mathrm{hg})_{\S 3}$, we have the identities

$$
g_{\delta}^{-}(-\delta)=g_{\delta}^{-}(a)=g_{\delta}^{-}(1-\delta)=0, \quad g_{\delta}^{+}(\delta)=g_{\delta}^{+}(a)=g_{\delta}^{+}(1+\delta)=0,
$$

together with the inequalities

$$
\begin{array}{ll}
g_{\delta}^{ \pm}(u)>0, & u \in(-\infty, \pm \delta) \cup(a, 1 \pm \delta) \\
g_{\delta}^{ \pm}(u)<0, & u \in( \pm \delta, a) \cup(1 \pm \delta, \infty) .
\end{array}
$$

(iii) We have the equalities

$$
D g_{\delta}^{-}(-\delta)=D g(0)=D g_{\delta}^{+}(+\delta), \quad D g_{\delta}^{-}(1-\delta)=D g(1)=D g_{\delta}^{+}(1+\delta)
$$

(iv) The maps $(u, \nu) \mapsto g_{\nu^{2}}^{ \pm}(u)$ are $C^{1}$-smooth. In addition, for fixed $\delta>0$ the maps $u \mapsto D g_{\delta}^{ \pm}(u)$ are locally Lipschitz. 
(v) There exists $\kappa_{\mathrm{dis}}>0$ such that we have

$$
g_{\delta}^{-}(u)-g(u) \leq-\kappa_{\mathrm{dis}} \delta
$$

for any $-\delta \leq u \leq 0$, together with

$$
g_{\delta}^{+}(u)-g(u) \geq \kappa_{\mathrm{dis}} \delta
$$

for any $1 \leq u \leq 1+\delta$.

Proof. Upon writing

$$
g_{\delta}^{-}(u)=g\left(\tau^{+}(u, \sqrt{\delta})\right), \quad g_{\delta}^{+}(u)=g\left(\tau^{-}(u, \sqrt{\delta})\right),
$$

the properties (i) through (iv) follow immediately from Lemma 3.8.

Addressing item (v), we note that there exists $\delta_{u}>0$ such that $g^{\prime}(u)<\frac{1}{2} g^{\prime}(0)<0$ for all $|u|<\delta_{u}$. In addition, since

$$
D g_{\delta}^{-}(u)=D g\left(\tau^{+}(u, \sqrt{\delta})\right) \partial_{u} \tau^{+}(u, \sqrt{\delta})
$$

with $u \leq \tau^{+}(u, \sqrt{\delta}) \leq u+\delta$ and $\frac{1}{2} \leq \partial_{u} \tau^{+}(u, \sqrt{\delta}) \leq 1$, we see that we can pick $\kappa^{\prime}>0$ in such a way that

$$
D g_{\delta}^{-}(u) \leq-\kappa^{\prime}, \quad D g(u) \leq-\kappa^{\prime}, \quad-\delta \leq u \leq 0,
$$

possibly after restricting $\delta>0$. We now find, for any $-\delta \leq u \leq 0$,

$$
g_{\delta}^{-}(u)-g(u)=D g_{\delta}^{-}\left(u_{1}\right)(u+\delta)-D g\left(u_{2}\right) u,
$$

for some $-\delta<u_{1}<u<u_{2}<0$. In particular, if $-\frac{\delta}{2} \leq u \leq 0$, we have

$$
g_{\delta}^{-}(u)-g(u) \leq D g_{\delta}^{-}\left(u_{1}\right)(u+\delta) \leq-\kappa^{\prime} \frac{\delta}{2},
$$

while if $-\delta \leq u \leq-\frac{\delta}{2}$ we have

$$
g_{\delta}^{-}(u)-g(u) \leq-D g\left(u_{2}\right) u \leq-\kappa^{\prime} \frac{\delta}{2} .
$$

The inequality for $g_{\delta}^{+}$follows analogously.

Linearizing the travelling wave MFDE (3.10) around the wave $(c, \Phi)$, we arrive at the homogeneous MFDE

$$
c v^{\prime}(\xi)=v\left(\xi+\sigma_{h}\right)+v\left(\xi-\sigma_{h}\right)+v\left(\xi+\sigma_{v}\right)+v\left(\xi-\sigma_{v}\right)-4 v(\xi)+g^{\prime}(\Phi(\xi)) v(\xi) .
$$

Our analysis in the remainder of this section hinges upon understanding solutions to (3.72) that decay at specified exponential rates on half-lines.

In particular, we choose four exponents $\eta_{\mathrm{fs}}^{ \pm}$and $\eta_{\mathrm{sl}}^{ \pm}$in such a way that

$$
0<\eta_{\mathrm{sl}}^{ \pm}<\eta_{\Phi}^{ \pm}<\eta_{\mathrm{fs}}^{ \pm}<2 \eta_{\mathrm{sl}}^{ \pm},
$$

while all non-real roots of $\Delta^{+}(z)=0$ have $\operatorname{Re} z \notin\left[-\eta_{\mathrm{fs}}^{+}, 0\right]$ and all non-real roots of $\Delta^{-}(z)=0$ have $\operatorname{Re} z \notin\left[0, \eta_{\mathrm{fs}}^{-}\right]$. Using these exponents, we introduce the constant

$$
\sigma=\max \left\{\left|\sigma_{h}\right|,\left|\sigma_{v}\right|\right\}
$$


together with the function spaces

$$
\begin{aligned}
& B C_{\mathrm{sl}}^{+}=\left\{v \in C([0, \infty), \mathbb{R}):\|v\|_{B C_{\mathrm{s} 1}^{+}}:=\sup _{\xi \geq 0} e^{\eta_{\mathrm{sl}}^{+}|\xi|}|v(\xi)|<\infty\right\}, \\
& B C_{\mathrm{sl}}^{-}=\left\{v \in C((-\infty, 0], \mathbb{R}):\|v\|_{B C_{\mathrm{sl}}^{-}}:=\sup _{\xi \leq 0} e^{\eta_{\mathrm{sl}}^{-}|\xi|}|v(\xi)|<\infty\right\}, \\
& B C_{1, \mathrm{sl}}^{\oplus}=\left\{v \in C([-\sigma, \infty), \mathbb{R}):\|v\|_{B C_{1, \mathrm{sl}}^{\oplus}}:=\sup _{\xi \geq-\sigma} e^{\eta_{\mathrm{sl}}^{+}|\xi|}\left[|v(\xi)|+\left|v^{\prime}(\xi)\right|\right]<\infty\right\}, \\
& B C_{1, \mathrm{sl}}^{\ominus}=\left\{v \in C((-\infty, \sigma], \mathbb{R}):\|v\|_{B C_{1, \mathrm{sl}}^{\ominus}}:=\sup _{\xi \leq \sigma} e^{\eta_{\mathrm{sl}}^{-}|\xi|}\left[|v(\xi)|+\left|v^{\prime}(\xi)\right|\right]<\infty\right\},
\end{aligned}
$$

with similar definitions for $B C_{\mathrm{fs}}^{ \pm}, B C_{1, \mathrm{fs}}^{\ominus}$ and $B C_{1, \mathrm{fs}}^{\oplus}$.

Returning to (3.72), we introduce the solution spaces

$$
\begin{aligned}
& \mathcal{P}_{\mathrm{fs}}=\left\{v \in B C_{1, \mathrm{fs}}^{\ominus}:\left[\mathcal{L}_{0} v\right](\xi)=0 \text { for all } \xi \leq 0\right\}, \\
& \mathcal{Q}_{\mathrm{fs}}=\left\{v \in B C_{1, \mathrm{fs}}^{\oplus}:\left[\mathcal{L}_{0} v\right](\xi)=0 \text { for all } \xi \geq 0\right\} .
\end{aligned}
$$

We note that we are abusing notation here in the sense that in addition to the definition (2.11), we are interpreting $\mathcal{L}_{0}$ as an operator from $B C_{1, \mathrm{fs}}^{\oplus} \rightarrow B C_{\mathrm{fs}}^{+}$and also as an operator from $B C_{1, \mathrm{fs}}^{\ominus} \rightarrow B C_{\mathrm{fs}}^{-}$.

In order to capture the initial conditions associated to the functions in the solution spaces (3.76), we will use the notation $\mathrm{ev}_{\xi} u \in C([-\sigma, \sigma], \mathbb{R})$ to denote the state of a continuous function $u$ at $\xi$, which is defined by

$$
\left[\operatorname{ev}_{\xi} u\right](\vartheta):=u(\xi+\vartheta), \quad \vartheta \in[-\sigma, \sigma] .
$$

This allows us to define the segment spaces

$$
\begin{aligned}
P_{\mathrm{fs}} & =\left\{\phi \in C([-\sigma, \sigma], \mathbb{R}): \phi=\operatorname{ev}_{0} v \text { for some } v \in \mathcal{P}_{\mathrm{fs}}\right\} \\
Q_{\mathrm{fs}} & =\left\{\phi \in C([-\sigma, \sigma], \mathbb{R}): \phi=\operatorname{ev}_{0} v \text { for some } v \in \mathcal{Q}_{\mathrm{fs}}\right\}, \\
B & =\operatorname{span}\left\{\operatorname{ev}_{0} \Phi^{\prime}\right\} .
\end{aligned}
$$

In view of the asymptotics (3.17)-(3.18), the fact that the kernel of $\mathcal{L}_{0}$ is one-dimensional implies that

$$
P_{\mathrm{fs}} \cap Q_{\mathrm{fs}}=\{0\}, \quad P_{\mathrm{fs}} \cap B=\{0\}, \quad Q_{\mathrm{fs}} \cap B=\{0\} .
$$

In order to obtain a splitting for the state space $C([-\sigma, \sigma], \mathbb{R})$ involving the components (3.78), we need to exploit the Hale inner product [22]. In the current setting, this bilinear form is given by

$$
\langle\psi, \phi\rangle:=c \psi(0) \phi(0)-\int_{0}^{\sigma} \psi(\theta-\sigma) \phi(\theta) \mathrm{d} \theta-\int_{0}^{-\sigma} \psi(\theta+\sigma) \phi(\theta) \mathrm{d} \theta
$$

for any pair $\phi, \psi \in C([-\sigma, \sigma], \mathbb{R})$. The Hale inner product is non-degenerate in the sense that if $\langle\psi, \phi\rangle=0$ for all $\psi \in C([-\sigma, \sigma], \mathbb{R})$, then necessarily $\phi=0$ [37]. As a consequence of [37, Thm. 4.3], we now have the characterization

$$
P_{\mathrm{fs}} \oplus Q_{\mathrm{fs}} \oplus B=\left\{\phi \in C([-\sigma, \sigma], \mathbb{R}) \mid\left\langle\mathrm{ev}_{0} \Psi, \phi\right\rangle=0\right\},
$$

in which we have recalled the function $\Psi$ defined by (2.18). Let us now pick a one-dimensional space $\Gamma \subset C([-\sigma, \sigma], \mathbb{R})$ that has the property that $\phi \in \Gamma$ satisfies $\phi=0$ if and only if $\left\langle\operatorname{ev}_{0} \Psi, \phi\right\rangle=0$. We now see that

$$
C([-\sigma, \sigma], \mathbb{R})=B \oplus Q_{\mathrm{fs}} \oplus P_{\mathrm{fs}} \oplus \Gamma .
$$

As customary, the solution $\Phi$ to the travelling wave equation (3.10) breaks when changing the parameters $\left(c, \sigma_{h}, \sigma_{v}\right)$. The key ingredient we will use in this section is that the arising gap can be captured in the finite dimensional space $\Gamma$. As a consequence, the size of such gaps can be measured effectively by means of the Hale inner product. This is particularly useful in view of the identity

$$
\frac{\mathrm{d}}{\mathrm{d} \xi}\left\langle\operatorname{ev}_{\xi} \Psi, \mathrm{ev}_{\xi} v\right\rangle=\Psi(\xi)\left[\mathcal{L}_{0} v\right](\xi)
$$


which holds for any pair $v \in C^{1}(\mathbb{R}, \mathbb{R})$ and $\xi \in \mathbb{R}$.

We now turn our attention to the perturbed linearization

$$
c^{\prime} v^{\prime}(\xi)=v\left(\xi+\sigma_{h}^{\prime}\right)+v\left(\xi-\sigma_{h}^{\prime}\right)+v\left(\xi+\sigma_{v}^{\prime}\right)+v\left(\xi-\sigma_{v}^{\prime}\right)-4 v(\xi)+g^{\prime}(\Phi(\xi)) v(\xi) .
$$

For convenience, we introduce the parameter $q^{\prime}=\left(c^{\prime}, \sigma_{h}^{\prime}, \sigma_{v}^{\prime}\right)$ and the set

$$
\mathcal{D}_{q}\left(\delta_{q}\right)=\left\{\left(c^{\prime}, \sigma_{h}^{\prime}, \sigma_{v}^{\prime}\right) \in \mathbb{R}^{3}:\left|c^{\prime}-c\right|+\left|\sigma_{h}^{\prime}-\sigma_{h}\right|+\left|\sigma_{v}^{\prime}-\sigma_{v}\right|<\delta_{q} \text { and } \sigma^{\prime}=\sigma\right\},
$$

in which we have introduced the notation

$$
\sigma^{\prime}=\max \left\{\left|\sigma_{h}^{\prime}\right|,\left|\sigma_{v}^{\prime}\right|\right\}
$$

We note that the restriction $\sigma^{\prime}=\sigma$ is a purely technical one in order to ensure that the state space $C([-\sigma, \sigma], \mathbb{R})$ remains unaffected. In light of the fact that (3.10) remains invariant under the transformations

$$
\xi \mapsto \lambda \xi, \quad\left(\sigma_{h}, \sigma_{v}\right) \rightarrow \lambda^{-1}\left(\sigma_{h}, \sigma_{v}\right), \quad c \mapsto \lambda c,
$$

this restriction will not hinder our ability to describe waves travelling in arbitrary directions sufficiently close to $\left(\sigma_{h}, \sigma_{v}\right)$.

For any $q^{\prime} \in \mathcal{D}_{q}\left(\delta_{q}\right)$, we introduce the differential operator $\mathcal{L}\left(q^{\prime}\right)$ that acts as

$$
\left[\mathcal{L}\left(q^{\prime}\right) v\right](\xi)=-c^{\prime} v^{\prime}(\xi)+v\left(\xi+\sigma_{h}^{\prime}\right)+v\left(\xi+\sigma_{v}^{\prime}\right)+v\left(\xi-\sigma_{h}^{\prime}\right)+v\left(\xi-\sigma_{v}^{\prime}\right)-4 v(\xi)+g^{\prime}(\Phi(\xi)) v(\xi) .
$$

As above, this operator will be interpreted as a linear map on both $B C_{1, \mathrm{fs}}^{\oplus}$ and $B C_{1, \mathrm{fs}}^{\ominus}$, mapping into $B C_{\mathrm{fs}}^{+}$and $B C_{\mathrm{fs}}^{-}$respectively.

We now borrow some convenient results from 31] that describe how $\mathcal{L}\left(q^{\prime}\right)$ and the spaces (3.78) vary with $q^{\prime}$. For explicitness, we write

$$
q_{*}=\left(c, \sigma_{h}, \sigma_{v}\right)
$$

Lemma 3.10 (see $[31, \S 5])$. Suppose that $(h g)_{\S 3}$ and $(h \Phi)_{\S 3}$ are both satisfied and pick $\delta_{q}>0$ sufficiently small. Then for any $q^{\prime}=\left(c^{\prime}, \sigma_{h}^{\prime}, \sigma_{v}^{\prime}\right) \in \mathcal{D}_{q}\left(\delta_{q}\right)$, there exist linear maps

$$
u_{Q_{\mathrm{fs}}}^{*}\left(q^{\prime}\right): Q_{\mathrm{fs}} \rightarrow B C_{1, \mathrm{fs}}^{\oplus}, \quad u_{P_{\mathrm{fs}}}^{*}\left(q^{\prime}\right): P_{\mathrm{fs}} \rightarrow B C_{1, \mathrm{fs}}^{\ominus}
$$

that satisfy the following properties.

(i) For any $\left(\phi_{Q}, \phi_{P}\right) \in Q_{\mathrm{fs}} \times P_{\mathrm{fs}}$ and $q^{\prime} \in \mathcal{D}_{q^{\prime}}\left(\delta_{q}\right)$, the function $v^{+}=u_{Q_{\mathrm{fs}}}^{*}\left(q^{\prime}\right) \phi_{Q}$ satisfies $\left[\mathcal{L}\left(q^{\prime}\right) v^{+}\right](\xi)=0$ for all $\xi \geq 0$, while $v^{-}=u_{P_{\mathrm{fs}}}^{*}\left(q^{\prime}\right) \phi_{P}$ satisfies $\left[\mathcal{L}\left(q^{\prime}\right) v^{-}\right](\xi)=0$ for all $\xi \leq 0$.

(ii) Pick any $q^{\prime} \in \mathcal{D}_{q}\left(\delta_{q}\right)$ and consider any pair $\left(v^{+}, v^{-}\right) \in B C_{1, \mathrm{fs}}^{\oplus} \times B C_{1, \mathrm{fs}}^{\ominus}$ for which $\left[\mathcal{L}\left(q^{\prime}\right) v^{+}\right](\xi)=$ 0 for all $\xi \geq 0$ and $\left[\mathcal{L}\left(q^{\prime}\right) v^{-}\right](\xi)=0$ for all $\xi \leq 0$ Then we must have

$$
v^{+}=u_{Q_{\mathrm{fs}}}^{*}\left(q^{\prime}\right) \Pi_{Q_{\mathrm{fs}}} \mathrm{ev}_{0} v^{+}, \quad v^{-}=u_{P_{\mathrm{fs}}}^{*}\left(q^{\prime}\right) \Pi_{P_{\mathrm{fs}}} \mathrm{ev}_{0} v^{-} .
$$

(iii) For any $q^{\prime} \in \mathcal{D}_{q}\left(\delta_{q}\right)$, we have the identities

$$
\Pi_{Q_{\mathrm{fs}}} \mathrm{ev}_{0} u_{Q_{\mathrm{fs}}}^{*}\left(q^{\prime}\right)=I, \quad \Pi_{P_{\mathrm{fs}}} \mathrm{ev}_{0} u_{P_{\mathrm{fs}}}^{*}\left(q^{\prime}\right)=I .
$$

(iv) The maps

$$
q^{\prime} \mapsto\left\{\begin{array}{l}
u_{Q_{\mathrm{fs}}}^{*}\left(q^{\prime}\right) \in \mathcal{L}\left(Q_{\mathrm{fs}}, B C_{1, \mathrm{fs}}^{\oplus}\right) \\
u_{P_{\mathrm{fs}}}^{*}\left(q^{\prime}\right) \in \mathcal{L}\left(P_{\mathrm{fs}}, B C_{1, \mathrm{fs}}^{\ominus}\right)
\end{array}\right.
$$

are $C^{1}$-smooth. 
Lemma 3.11 (see [31, §3] ). Suppose that $(h g)_{\S 3}$ and $(h \Phi)_{\S 3}$ are both satisfied and pick $\delta_{q}>0$ sufficiently small. Then for any $q^{\prime}=\left(c^{\prime}, \sigma_{h}^{\prime}, \sigma_{v}^{\prime}\right) \in \mathcal{D}_{q}\left(\delta_{q}\right)$, there exist linear maps

$$
\mathcal{L}_{\text {inv }}^{+}\left(q^{\prime}\right): B C_{\mathrm{fs}}^{+} \rightarrow B C_{1, \mathrm{fs}}^{\oplus}, \quad \mathcal{L}_{\mathrm{inv}}^{-}\left(q^{\prime}\right): B C_{\mathrm{fs}}^{-} \rightarrow B C_{1, \mathrm{fs}}^{\ominus}
$$

that satisfy the following properties.

(i) For every $f^{ \pm} \in B C_{\mathrm{fs}}^{ \pm}$and $q^{\prime} \in \mathcal{D}_{q}\left(\delta_{q}\right)$, the function $v^{+}=\mathcal{L}_{\text {inv }}^{+}\left(q^{\prime}\right) f^{+}$satisfies $\left[\mathcal{L}\left(q^{\prime}\right) v^{+}\right](\xi)=$ $f^{+}(\xi)$ for all $\xi \geq 0$, while $v^{-}=\mathcal{L}_{\text {inv }}^{-}\left(q^{\prime}\right) f^{-}$satisfies $\left[\mathcal{L}\left(q^{\prime}\right) v^{-}\right](\xi)=f^{-}(\xi)$ for all $\xi \leq 0$.

(ii) For every $f^{ \pm} \in B C_{\mathrm{fs}}^{ \pm}$and $q^{\prime} \in \mathcal{D}_{q}\left(\delta_{q}\right)$, we have the identities

$$
\begin{aligned}
& \Pi_{Q_{\mathrm{fs}}} \mathrm{ev}_{0} \mathcal{L}_{\mathrm{inv}}^{+}\left(q^{\prime}\right) f^{+}=0, \\
& \Pi_{P_{\mathrm{fs}}} \mathrm{ev}_{0} \mathcal{L}_{\mathrm{inv}}^{-}\left(q^{\prime}\right) f^{-}=0 .
\end{aligned}
$$

(iii) The maps

$$
q^{\prime} \mapsto\left\{\begin{array}{l}
\mathcal{L}_{\text {inv }}^{+}\left(q^{\prime}\right) \in \mathcal{L}\left(B C_{\mathrm{fs}}^{+}, B C_{1, \mathrm{fs}}^{\oplus}\right) \\
\mathcal{L}_{\text {inv }}^{-}\left(q^{\prime}\right) \in \mathcal{L}\left(B C_{\mathrm{fs}}^{-}, B C_{1, \mathrm{fs}}^{\ominus}\right)
\end{array}\right.
$$

are $C^{1}$-smooth.

The next result can be seen as a continuation result for the two halves of the wave profile $\Phi^{\prime}$ upon varying $q$. In particular, we construct two solution families for the homogeneous MFDE (3.84) that decay at the relevant slow exponential rate. This will allow us to control the constants appearing in the asymptotic expansions (3.17)-(3.18).

Lemma 3.12. Suppose that $(\mathrm{hg})_{\S 3}$ and $(h \Phi)_{\S 3}$ are both satisfied and pick $\delta_{q}>0$ sufficiently small. Then for any $q^{\prime}=\left(c^{\prime}, \sigma_{h}^{\prime}, \sigma_{v}^{\prime}\right) \in \mathcal{D}_{q}\left(\delta_{q}\right)$, there exist functions

$$
b^{+}=b^{+}\left(q^{\prime}\right) \in B C_{1, \mathrm{sl}}^{\oplus}, \quad b^{-}=b^{-}\left(q^{\prime}\right) \in B C_{1, \mathrm{sl}}^{\ominus}
$$

that satisfy the following properties.

(i) We have $\left[\mathcal{L}\left(q^{\prime}\right) b^{+}\left(q^{\prime}\right)\right](\xi)=0$ for all $\xi \geq 0$, together with $\left[\mathcal{L}\left(q^{\prime}\right) b^{-}\left(q^{\prime}\right)\right](\xi)=0$ for all $\xi \leq 0$.

(ii) The maps $q^{\prime} \mapsto b^{+}\left(q^{\prime}\right) \in B C_{1, \mathrm{sl}}^{\oplus}$ and $q^{\prime} \mapsto b^{-}\left(q^{\prime}\right) \in B C_{1, \mathrm{sl}}^{\ominus}$ are $C^{1}$-smooth, with $b^{ \pm}\left(q_{*}\right)=\Phi^{\prime}$.

(iii) For all $q^{\prime} \in \mathcal{D}_{q}\left(\delta_{q}\right)$ we have $\Pi_{B} \mathrm{ev}_{0} b^{ \pm}\left(q^{\prime}\right)=\mathrm{ev}_{0} \Phi^{\prime}$, together with

$$
\Pi_{Q_{\mathrm{fs}}} \mathrm{ev}_{0} b^{+}\left(q^{\prime}\right)=0, \quad \Pi_{P_{\mathrm{fs}}} \mathrm{ev}_{0} b^{-}\left(q^{\prime}\right)=0 .
$$

(iv) Upon writing $\eta_{q^{\prime}}^{+}>0$ for the exponent defined in Lemma 3.3 applied to the characteristic equation

$$
\Delta_{q^{\prime}}^{+}(z)=c^{\prime} z-\left(2 \cosh \left(\sigma_{h}^{\prime} z\right)+2 \cosh \left(\sigma_{v}^{\prime} z\right)-4\right)-g^{\prime}(1)
$$

and similarly defining $\eta_{q^{\prime}}^{-}>0$, there exist constants $C_{q^{\prime}}^{ \pm}>0$ and $K_{2}>1$ such that for all $q^{\prime} \in \mathcal{D}_{q}\left(\delta_{q}\right)$ we have

$$
\begin{array}{ll}
\left|b^{+}\left(q^{\prime}\right)(\xi)-C_{q^{\prime}}^{+} e^{-\eta_{q^{\prime}}^{+}|\xi|}\right| \leq K_{2} e^{-\eta_{\mathrm{fs}}^{+}|\xi|}, \quad \xi \geq 0, \\
\left|b^{-}\left(q^{\prime}\right)(\xi)-C_{q^{\prime}}^{-} e^{-\eta_{q^{\prime}}^{-}|\xi|}\right| \leq K_{2} e^{-\eta_{\mathrm{fs}}^{-}|\xi|}, \quad \xi \leq 0 .
\end{array}
$$

In addition, the maps $q^{\prime} \mapsto C_{q^{\prime}}^{ \pm}$are $C^{1}$-smooth. 
Proof. Since the maps $q^{\prime} \mapsto \eta_{q^{\prime}}^{+}$are $C^{1}$-smooth, we can construct a map $q^{\prime} \mapsto b_{0}^{+}\left(q^{\prime}\right)$ that satisfies conditions (ii) - (iv) simply by using

$$
b_{0}^{+}\left(q^{\prime}\right)(\xi)=\Phi^{\prime}\left(\left[\eta_{q^{\prime}}^{+} / \eta_{\Phi}^{+}\right] \xi\right), \quad \xi \gg 1
$$

and ensuring that $b_{0}^{+}\left(q^{\prime}\right)(\xi)=\Phi^{\prime}(\xi)$ for $\xi \in[-\sigma, \sigma]$.

We now write $b^{+}\left(q^{\prime}\right)=b_{0}^{+}\left(q^{\prime}\right)+v\left(q^{\prime}\right)$ and find that (i) now requires that the function $v\left(q^{\prime}\right)$ satisfy

$$
\left[\mathcal{L}\left(q^{\prime}\right) v\left(q^{\prime}\right)\right](\xi)=f_{q^{\prime}}(\xi):=-\left[\mathcal{L}\left(q^{\prime}\right) b_{0}^{+}\left(q^{\prime}\right)\right](\xi), \quad \xi \geq 0 .
$$

By construction however, we see that $f_{q^{\prime}} \in B C_{\mathrm{fs}}^{+}$, which allows us to write

$$
v\left(q^{\prime}\right)=\mathcal{L}_{\text {inv }}^{+}\left(q^{\prime}\right) f_{q^{\prime}} \in B C_{1, \mathrm{fs}}^{\oplus},
$$

which depends $C^{1}$-smoothly on $q^{\prime}$ and has $v\left(q_{*}\right)=0$. This ensures that (ii) and (iv) remain satisfied. In addition, a simple multiplicative rescaling allows (iii) to be restored.

Based on the ingredients above, we can follow the procedure developed in [31] to implement a version of Lin's method. In particular, we combine the two inverses $\mathcal{L}_{\text {inv }}^{ \pm}\left(q^{\prime}\right)$ to construct solutions to $\mathcal{L}\left(q^{\prime}\right) v=f$ up to a gap at zero, which can be contained in the one-dimensional space $\Gamma$.

Lemma 3.13 (see [28, Lem. 5.10]). Suppose that $(h g)_{\S 3}$ and $(h \Phi)_{\S 3}$ are both satisfied and pick $\delta_{q}>0$ sufficiently small. Then for any $q^{\prime}=\left(c^{\prime}, \sigma_{h}^{\prime}, \sigma_{v}^{\prime}\right) \in \mathcal{D}_{q}\left(\delta_{q}\right)$ and any pair $\left(f^{-}, f^{+}\right) \in B C_{\mathrm{fs}}^{-} \times B C_{\mathrm{fs}}^{+}$, there is a unique quadruplet

$$
\left(v^{-}, \alpha^{-}, v^{+}, \alpha^{+}\right) \in B C_{1, \mathrm{fs}}^{\ominus} \times \mathbb{R} \times B C_{1, \mathrm{fs}}^{\oplus} \times \mathbb{R}
$$

for which the pair

$$
w^{-}\left(q^{\prime}\right)=v^{-}\left(q^{\prime}\right)+\alpha^{-}\left(q^{\prime}\right) b^{-}\left(q^{\prime}\right) \in B C_{1, \mathrm{sl}}^{\ominus}, \quad w^{+}\left(q^{\prime}\right)=v^{+}\left(q^{\prime}\right)+\alpha^{+}\left(q^{\prime}\right) b^{+}\left(q^{\prime}\right) \in B C_{1, \mathrm{sl}}^{\oplus}
$$

satisfies the following properties.

(i) For all $\xi \geq 0$ we have $\left[\mathcal{L}\left(q^{\prime}\right) v^{-}\right](\xi)=\left[\mathcal{L}\left(q^{\prime}\right) w^{-}\right](\xi)=f^{-}(\xi)$, while for all $\xi \geq 0$ we have $\left[\mathcal{L}\left(q^{\prime}\right) v^{+}\right](\xi)=\left[\mathcal{L}\left(q^{\prime}\right) w^{+}\right](\xi)=f^{+}(\xi)$.

(ii) We have the inclusions $\mathrm{ev}_{0} w^{ \pm} \in P_{\mathrm{fs}} \oplus Q_{\mathrm{fs}} \oplus B$.

(iii) The gap between $w^{+}$and $w^{-}$at zero satisfies $\mathrm{ev}_{0}\left[w^{+}-w^{-}\right] \in \Gamma$.

Upon writing

$$
\left(v^{-}, \alpha^{-}, v^{+}, \alpha^{+}\right)=L_{3}\left(q^{\prime}\right)\left(f^{-}, f^{+}\right)
$$

for the quadruplet described above, the map

$$
q^{\prime} \mapsto L_{3}\left(q^{\prime}\right) \in \mathcal{L}\left(B C_{\mathrm{fs}}^{-} \times B C_{\mathrm{fs}}^{+}, B C_{1, \mathrm{fs}}^{\ominus} \times \mathbb{R} \times B C_{1, \mathrm{fs}}^{\oplus} \times \mathbb{R}\right)
$$

is $C^{1}$-smooth. In addition, the gap at zero satisfies the identity

$$
\left\langle\operatorname{ev}_{0} \Psi, \operatorname{ev}_{0}\left[w^{+}-w^{-}\right]\right\rangle=\int_{-\infty}^{0} \Psi(\xi)\left[\mathcal{L}\left(q_{*}\right) w^{-}\right](\xi) d \xi+\int_{0}^{\infty} \Psi(\xi)\left[\mathcal{L}\left(q_{*}\right) w^{+}\right](\xi) d \xi .
$$

We are now ready to construct a solution to the MFDE

$$
\begin{aligned}
c^{\prime} W^{\prime}(\xi)=W(\xi & \left.+\sigma_{h}^{\prime}\right)+W\left(\xi+\sigma_{v}^{\prime}\right)+W\left(\xi-\sigma_{h}^{\prime}\right)+W\left(\xi-\sigma_{v}^{\prime}\right)-4 W(\xi) \\
& +g_{\delta}^{-}(W(\xi))
\end{aligned}
$$


on half-lines. We note that the asymptotic estimates (3.17)-(3.18) allow us to write

$$
\begin{aligned}
& \Phi(\xi)=v_{*}^{-}+\left[\eta_{\Phi}^{-}\right]^{-1} \Phi^{\prime}(\xi), \quad \xi \leq \sigma, \\
& \Phi(\xi)=1+v_{*}^{+}-\left[\eta_{\Phi}^{+}\right]^{-1} \Phi^{\prime}(\xi), \quad \xi \geq-\sigma,
\end{aligned}
$$

for some pair $\left(v_{*}^{-}, v_{*}^{+}\right) \in B C_{1, \mathrm{fs}}^{\ominus} \times B C_{1, \mathrm{fs}}^{\oplus}$. Introducing the notation

$$
h^{-}=\left(v^{-}, \alpha^{-}\right) \in B C_{1, \mathrm{fs}}^{\ominus} \times \mathbb{R}, \quad h^{+}=\left(v^{+}, \alpha^{+}\right) \in B C_{1, \mathrm{fs}}^{\oplus} \times \mathbb{R},
$$

we fix $\delta>0$ and define the functions

$$
\begin{aligned}
& {\left[W^{-}\left(h^{-}\right)\right](\xi)=-\delta+v_{*}^{-}+v^{-}+\left(\alpha^{-}+\left[\eta_{\Phi}^{-}\right]^{-1}\right) b^{-}\left(q^{\prime}\right) \quad \xi \leq \sigma,} \\
& {\left[W^{+}\left(h^{+}\right)\right](\xi)=1-\delta+v_{*}^{+}+v^{+}+\left(\alpha^{+}-\left[\eta_{\Phi}^{+}\right]^{-1}\right) b^{+}\left(q^{\prime}\right) \quad \xi \geq-\sigma .}
\end{aligned}
$$

We intend to find a pair $\left(h^{-}, h^{+}\right)$such that (3.109) with $W=W^{+}\left(h^{+}\right)$is satisfied for $\xi \geq 0$, while (3.109) with $W=W^{-}\left(h^{-}\right)$is satisfied for $\xi \leq 0$. Plugging this Ansatz into (3.109), we find

$$
\begin{aligned}
-\left[\mathcal{L}\left(q^{\prime}\right) v^{-}\right](\xi) & =\mathcal{R}_{q^{\prime}, \delta}^{-}\left(h^{-} ; \xi\right), & & \xi \leq 0, \\
-\left[\mathcal{L}\left(q^{\prime}\right) v^{+}\right](\xi) & =\mathcal{R}_{q^{\prime}, \delta}^{+}\left(h^{+} ; \xi\right), & & \xi \geq 0,
\end{aligned}
$$

with nonlinear terms

$$
\begin{aligned}
\mathcal{R}_{q^{\prime}, \delta}^{-}\left(h^{-} ; \xi\right)=(c- & \left.c^{\prime}\right)\left[v_{*}^{-}\right]^{\prime}(\xi)+v_{*}^{-}\left(\xi+\sigma_{h}^{\prime}\right)+v_{*}^{-}\left(\xi-\sigma_{h}^{\prime}\right)-v_{*}^{-}\left(\xi+\sigma_{h}\right)-v_{*}^{-}\left(\xi-\sigma_{h}\right) \\
& +v_{*}^{-}\left(\xi+\sigma_{v}^{\prime}\right)+v_{*}^{-}\left(\xi-\sigma_{v}^{\prime}\right)-v_{*}^{-}\left(\xi+\sigma_{v}\right)-v_{*}^{-}\left(\xi-\sigma_{v}\right) \\
& +g_{\delta}^{-}\left(W^{-}\left(h^{-}\right)\right)-g\left(\delta+W^{-}\left(h^{-}\right)\right) \\
& +\mathcal{R}_{0}\left(v^{-}(\xi)+\alpha^{-} b^{-}\left(q^{\prime}\right)(\xi)+\left[\eta_{\Phi}^{-}\right]^{-1}\left[b^{-}\left(q^{\prime}\right)(\xi)-\Phi^{\prime}(\xi)\right] ; \xi\right), \\
\mathcal{R}_{q^{\prime}, \delta}^{+}\left(h^{+} ; \xi\right)=(c- & \left.c^{\prime}\right)\left[v_{*}^{+}\right]^{\prime}(\xi)+v_{*}^{+}\left(\xi+\sigma_{h}^{\prime}\right)+v_{*}^{+}\left(\xi-\sigma_{h}^{\prime}\right)-v_{*}^{+}\left(\xi+\sigma_{h}\right)-v_{*}^{+}\left(\xi-\sigma_{h}\right) \\
& +v_{*}^{+}\left(\xi+\sigma_{v}^{\prime}\right)+v_{*}^{+}\left(\xi-\sigma_{v}^{\prime}\right)-v_{*}^{+}\left(\xi+\sigma_{v}\right)-v_{*}^{+}\left(\xi-\sigma_{v}\right) \\
& +g_{\delta}^{-}\left(W^{+}\left(h^{+}\right)\right)-g\left(\delta+W^{+}\left(h^{+}\right)\right) \\
& +\mathcal{R}_{0}\left(v^{+}(\xi)+\alpha^{+} b^{+}\left(q^{\prime}\right)(\xi)-\left[\eta_{\Phi}^{+}\right]^{-1}\left[b^{+}\left(q^{\prime}\right)(\xi)-\Phi^{\prime}(\xi)\right] ; \xi\right),
\end{aligned}
$$

in which we have introduced the expression

$$
\mathcal{R}_{0}(v ; \xi)=g(\Phi(\xi)+v)-g(\Phi(\xi))-g^{\prime}(\Phi(\xi)) v .
$$

Lemma 3.14. Suppose that $(h g)_{\S 3}$ and $(h \Phi)_{\S 3}$ are both satisfied, pick sufficiently small constants $\delta_{q}>0$ and $\delta_{0}>0$ and an arbitrary constant $M_{4}>1$. Then there exists a constant $C_{4}>1$ and an exponent $\kappa_{4}>0$ such that for any sets

$$
\left(v^{+}, v_{1}^{+}, v_{2}^{+}\right) \in\left[B C_{1, \mathrm{fs}}^{\oplus}\right]^{3}, \quad\left(\alpha^{+}, \alpha_{1}^{+}, \alpha_{2}^{+}\right) \in \mathbb{R}^{3}
$$

that have

$$
\left\|v^{+}\right\|_{B C_{1, \mathrm{fs}}^{\oplus}}+\left\|v_{1}^{+}\right\|_{B C_{1, \mathrm{fs}}^{\oplus}}+\left\|v_{2}^{+}\right\|_{B C_{1, \mathrm{fs}}^{\oplus}} \leq M_{4}, \quad\left|\alpha^{+}\right|+\left|\alpha_{1}^{+}\right|+\left|\alpha_{2}^{+}\right| \leq M_{4},
$$

any $0 \leq \delta<\delta_{0}$ and any $q^{\prime} \in \mathcal{D}_{q}\left(\delta_{q}\right)$, we have the estimates

$$
\left\|\mathcal{R}_{q^{\prime}, \delta}^{+}\left(v^{+}, \alpha^{+} ; \cdot\right)\right\|_{B C_{\mathrm{fs}}^{+}} \leq C_{4} \delta^{\kappa_{4}}+C_{4}\left|q^{\prime}-q_{*}\right|+C_{4}\left[\left|\alpha^{+}\right|+\left\|v^{+}\right\|_{B C_{1, \mathrm{fs}}^{\oplus}}\right]^{2},
$$


together with

$$
\left\|\mathcal{R}_{q^{\prime}, \delta}^{+}\left(v_{1}^{+}, \alpha_{1}^{+} ; \cdot\right)-\mathcal{R}_{q^{\prime}, \delta}^{+}\left(v_{2}^{+}, \alpha_{2}^{+} ; \cdot\right)\right\|_{B C_{\mathrm{fs}}^{+}} \leq \begin{gathered}
C_{4}[ \\
{[\delta}
\end{gathered}
$$

Similar estimates hold for the nonlinearities $\mathcal{R}_{q^{\prime}, \delta}^{-}$.

Proof. Notice first that for every $u \in \mathbb{R}$, there exists $0<\vartheta<1$ so that

$$
\tau^{+}(u-\delta, \sqrt{\delta})-u=\left[\partial_{u} \tau^{+}(-\delta+\vartheta u, \sqrt{\delta})-\partial_{u} \tau^{+}(-\delta, \sqrt{\delta})\right] u .
$$

Using the Lipschitz property (v) obtained in Lemma 3.8 for $\partial_{u} \tau^{+}$, together with the bound (3.46), one finds that there exists $C_{1}^{\prime}>1$ so that

$$
\left|g_{\delta}^{-}(-\delta+u)-g(u)\right| \leq C_{1}^{\prime} \min \{\sqrt{\delta},|u|\}|u|
$$

holds for all $u \in \mathbb{R}$ and all $0 \leq \delta<\delta_{0}$. In a similar fashion, for all such $u$ and $\delta$ we have

$$
\left|g_{\delta}^{-}(-\delta+u)-g(u)\right| \leq C_{1}^{\prime} \min \{\sqrt{\delta},|1-u|\}|1-u| .
$$

In particular, whenever $1-u \in B C_{\mathrm{sl}}^{+}$, we can estimate

$$
\left\|g_{\delta}(-\delta+u(\cdot))-g(u(\cdot))\right\|_{B C_{\mathrm{fs}}^{+}} \leq C_{1}^{\prime} \delta^{\frac{2 \eta_{\mathrm{sl}}^{+}-\eta_{\mathrm{fs}}^{+}}{2 \eta_{\mathrm{sl}}^{+}}}\|1-u\|_{B C_{\mathrm{sl}}^{+}}
$$

In addition, exploiting the Lipschitz continuity of $g^{\prime}$, for any $u \in B C_{\mathrm{sl}}^{+}$one easily estimates

$$
\left\|\mathcal{R}_{0}(u(\cdot) ; \cdot)\right\|_{B C_{\mathrm{fs}}^{+}} \leq C_{2}^{\prime}\|u\|_{B C_{\mathrm{sl}}^{+}}^{2},
$$

for some $C_{2}^{\prime}>1$, which suffices to establish (3.118). The Lipschitz bound (3.119) can be obtained using standard arguments, again exploiting the Lipschitz continuity of $g^{\prime}$ and $\partial_{u} \tau^{+}$.

Proof of Proposition 3.7. Fix $\delta_{0}>0$ and $\delta_{q}>0$ sufficiently small. On account of the estimates in Lemma 3.14, a fixed point argument can be used to construct for any $q^{\prime} \in \mathcal{D}_{q}\left(\delta_{q}\right)$ and $0 \leq \delta<\delta_{0}$, a pair $\left(h_{*}^{-}, h_{*}^{+}\right)=\left(h_{*}^{-}, h_{*}^{+}\right)\left(q^{\prime}, \delta\right)$ with

$$
\left(h_{*}^{-}, h_{*}^{+}\right)=L_{3}\left(q^{\prime}\right)\left(\mathcal{R}_{q^{\prime}, \delta}^{-}\left(h_{*}^{-} ; \cdot\right), \mathcal{R}_{q^{\prime}, \delta}^{+}\left(h_{*}^{+} ; \cdot\right)\right) .
$$

In addition, the map $\left(q^{\prime}, \nu\right) \mapsto\left(h_{*}^{-}, h_{*}^{+}\right)\left(q^{\prime}, \nu^{2}\right)$ is $C^{1}$-smooth with $\left(h_{*}^{-}, h_{*}^{+}\right)(q, 0)=0$. The functions $W^{+}\left(h_{*}^{+}\right)$and $W^{-}\left(h_{*}^{-}\right)$together define a solution to the travelling wave system (3.109) provided $\mathrm{ev}_{0}\left[W^{+}\left(h_{*}^{+}\right)-W^{-}\left(h_{*}^{-}\right)\right]=0 \in \Gamma$. This one dimensional equation implicitly defines $c^{\prime}$ as a $C^{1}-$ smooth function of $\left(\sqrt{\delta}, \sigma_{h}^{\prime}, \sigma_{v}^{\prime}\right)$, which can be seen by exploiting (3.108) and using the identity $\int_{\mathbb{R}} \Psi(\xi) \Phi^{\prime}(\xi) d \xi=1$ to verify the conditions of the implicit function theorem. Similar computations can be found in [27]. This concludes the construction of the pairs $\left(c_{p}^{-}, \Phi_{p}^{-}\right)$.

The pairs $\left(c_{p}^{+}, \Phi_{p}^{+}\right)$can be constructed in a similar fashion and the differential inequalities (3.28) now follow from the identities

$$
\mathcal{J}_{i j}^{ \pm}(t)=g_{\delta}^{ \pm}\left(W_{i j}^{ \pm}(t)\right)-g\left(W_{i j}^{ \pm}(t)\right),
$$

together with the inequalities (3.60). 


\section{Spreading Speed}

In this section we consider the homogeneous lattice and set out to prove that large disturbances from the zero rest state spread out to fill the entire lattice $\mathbb{Z}^{2}$, provided the initial support of the disturbance is sufficiently large. This is the analogue of the classic result [1, Thm. 5.3] obtained by Aronson and Weinberger for bistable reaction-diffusion PDEs.

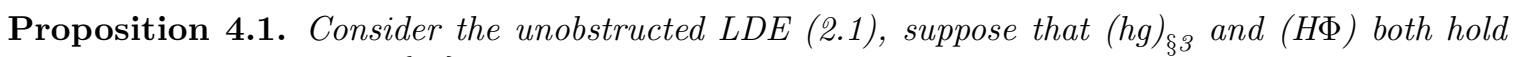
and write $c_{*}=\min _{\zeta \in[0,2 \pi]}\left\{c_{\zeta}\right\}$. Fix any $0<c<c_{*}$. Then for any sufficiently small $\eta>0$, there exist $R=R(c, \eta)>0$ and $T=T(c, \eta)>0$ such that the solution to the $L D E$ (2.1) with initial condition

$$
u_{i j}(0)= \begin{cases}1-\eta & \sqrt{i^{2}+j^{2}} \leq R \\ 0 & \left|i^{2}+j^{2}\right|>R\end{cases}
$$

satisfies $u_{i j}(t) \geq 1-\eta$ for all $t \geq T$ and $\sqrt{i^{2}+j^{2}} \leq R+c(t-T)$.

The main difficulty here is that it is no longer possible to construct a radially symmetric expanding sub-solution, because the wave profiles are angular dependent. In addition, the technical trick [1, (5.8)], which allowed a smooth flat core to be connected to an outwardly travelling wave via a sharp interface, is no longer available. Instead, we work here with suitably stretched versions of the angular dependent wave profiles in order to construct a wide transition area between the core and the outgoing waves.

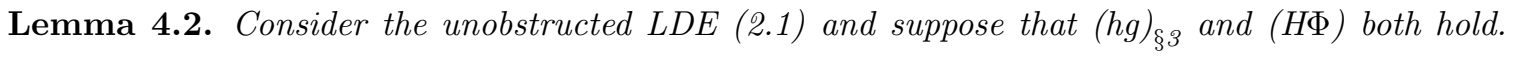
Pick a sufficiently small $\delta_{0}>0$ and recall the nonlinearities $g_{\delta}^{-}$defined in Lemma 3.9. Then for all $0 \leq \delta<\delta_{0}$ and $\zeta \in[0,2 \pi]$ there exists a wave speed $c_{\zeta ; \delta}>0$ together with a wave profile $\Phi_{\zeta ; \delta} \in C^{1}(\mathbb{R}, \mathbb{R})$ that satisfies the $M F D E$

$$
\begin{gathered}
c \Phi_{\zeta ; \delta}^{\prime}(\xi)=\Phi_{\zeta ; \delta}(\xi+\cos \zeta)+\Phi_{\zeta ; \delta}(\xi+\sin \zeta)+\Phi_{\zeta ; \delta}(\xi-\cos \zeta)+\Phi_{\zeta ; \delta}(\xi-\sin \zeta)-4 \Phi_{\zeta ; \delta}(\xi) \\
+g_{\delta}^{-}\left(\Phi_{\zeta ; \delta}(\xi)\right)
\end{gathered}
$$

and enjoys the limits

$$
\lim _{\xi \rightarrow-\infty} \Phi_{\zeta ; \delta}(\xi)=-\delta, \quad \lim _{\xi \rightarrow+\infty} \Phi_{\zeta ; \delta}(\xi)=1-\delta .
$$

In addition, there exists a constant $C_{1}>1$ so that for all $0 \leq \delta<\delta_{0}$ and $\zeta \in[0,2 \pi]$, we have the uniform bound

$$
\frac{\left|\Phi_{\zeta ; \delta}^{\prime}\left(\xi^{\prime}\right)\right|}{\left|\Phi_{\zeta ; \delta}^{\prime}(\xi)\right|} \leq C_{1}, \quad\left|\xi^{\prime}-\xi\right| \leq 2
$$

Proof. The statements follow directly from Proposition 3.7. In particular, the uniform bound (4.4) follows from the continuity properties stated in item (v) of this result.

In order to connect the waves defined above to a flat inner core, we need to ensure that the wave profiles are all cut off at the same value. In addition, we need to enforce a convexity condition in the transition area. The next result handles these two requirements.

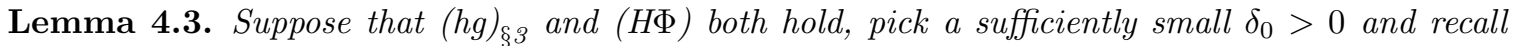
the wave profiles $\Phi_{\zeta ; \delta}$ defined in Lemma 4.2. Then for any $h_{\infty} \geq 0$, there exists a continuous map $\tau_{h_{\infty}}:[0,2 \pi] \times\left(0, \delta_{0}\right) \rightarrow \mathbb{R}$ and a continuous map $\Phi_{\infty ; h_{\infty}}:\left(0, \delta_{0}\right) \rightarrow\left(1-2 \delta_{0}, 1\right)$, such that the shifted profiles

$$
\Phi_{\zeta ; \delta, h_{\infty}}(\xi):=\Phi_{\zeta ; \delta}\left(\xi+\tau_{h_{\infty}}(\zeta, \delta)\right)
$$

satisfy the following properties. 
(i) For any $\delta \in\left(0, \delta_{0}\right)$, we have

$$
\Phi_{\zeta ; \delta, h_{\infty}}^{\prime \prime}(\xi) \leq 0, \quad \xi \geq 0, \quad 0 \leq \zeta \leq 2 \pi .
$$

(ii) For any $\delta \in\left(0, \delta_{0}\right)$, we have

$$
\Phi_{\zeta ; \delta, h_{\infty}}(0) \geq 1-2 \delta, \quad 0 \leq \zeta \leq 2 \pi .
$$

(iii) For any $\delta \in\left(0, \delta_{0}\right)$ and $0 \leq \zeta \leq 2 \pi$, we have

$$
\Phi_{\zeta ; \delta, h_{\infty}}\left(h_{\infty}\right)=\Phi_{\infty ; h_{\infty}}(\delta) \geq 1-2 \delta .
$$

(iv) For any fixed $0<\delta<\delta_{0}$, the map $\zeta \mapsto \tau_{h_{\infty}}(\zeta, \delta)$ is $C^{1}$-smooth.

Proof. We note first that the asymptotic estimates in Proposition 3.4 imply that the wave profiles $\Phi_{\zeta ; \delta}(\xi)$ are convex down as $\xi \rightarrow+\infty$. In particular, the continuity properties of the asymptotic coefficients that are stated in item (v) of Proposition 3.7 allow us to construct a continuous function $\tau_{*}:\left(0, \delta_{0}\right) \rightarrow \mathbb{R}$ in such a way that for all $\zeta \in[0,2 \pi]$ and all $0<\delta<\delta_{0}$, we have the inequalities

$$
\Phi_{\zeta ; \delta}^{\prime \prime}\left(\xi+\tau_{*}(\delta)\right) \leq 0, \quad \xi \geq 0,
$$

together with

$$
\Phi_{\zeta ; \delta}\left(\tau_{*}(\delta)\right) \geq 1-2 \delta .
$$

Now, for every $0<\delta<\delta_{0}$ we define

$$
\Phi_{\infty ; h_{\infty}}(\delta)=\max _{\zeta \in[0,2 \pi]} \Phi_{\zeta ; \delta}\left(\tau_{*}(\delta)+h_{\infty}\right),
$$

which depends continuously on $\delta$. We can now pick $\tau_{h_{\infty}}(\zeta, \delta)$ in such a way that

$$
\Phi_{\zeta ; \delta}\left(\tau_{h_{\infty}}(\zeta, \delta)\right)=\Phi_{\infty ; h_{\infty}}(\delta)
$$

holds for all $\zeta \in[0,2 \pi]$, which establishes (iii). Since $\Phi_{\zeta ; \delta}^{\prime}>0$, we necessarily have $\tau_{h_{\infty}}(\delta, \zeta) \geq \tau_{*}(\delta)$, which implies (i) and (ii).

The smoothness (iv) can be established by noting that for any $\alpha$ in the range of $\Phi_{\zeta ; \nu^{2}}$, the implicit definition

$$
\Phi_{\zeta ; \nu^{2}}(\xi)=\alpha
$$

locally defines a $C^{1}$-smooth function $\xi=\xi(\alpha, \zeta, \nu)$ on account of the fact that $\Phi_{\zeta ; \delta}^{\prime}>0$. This observation also implies that the continuity of the map $(\zeta, \delta) \mapsto \tau_{h_{\infty}}(\zeta, \delta)$ follows directly from the continuity of $\delta \mapsto \Phi_{\infty ; h_{\infty}}(\delta)$.

Our next result provides a bound on the angular derivatives of the wave profiles. We emphasize that the constants $K_{2}$ below cannot be taken to be uniform across $0 \leq \delta<\delta_{0}$, because the shifts $\tau_{h_{\infty}}$ defined above generically become unbounded as $\delta \rightarrow 0$.

Corollary 4.4. Suppose that $(\mathrm{hg})_{\S 3}$ and $(H \Phi)$ both hold and pick a sufficiently small $\delta_{0}>0$. Then there exists an exponent $\eta_{*}>0$ so that the following holds true.

For any $h_{\infty} \geq 0$ and any $0<\delta<\delta_{0}$, there exists a constant $K_{2}=K_{2}\left(\delta ; h_{\infty}\right)>1$ such that the estimates

$$
\partial_{\zeta} \Phi_{\zeta ; \delta, h_{\infty}}(\xi) \leq K_{2} e^{-\eta_{*}|\xi|}, \quad \xi \in \mathbb{R}, \quad \zeta \in[0,2 \pi],
$$

hold for the wave profiles defined in Lemma 4.3.

Proof. The existence of the derivative with respect to $\zeta$ follows from item (iv) of Lemma 4.3, together with the smoothness properties established in item (iv) of Proposition 3.7. The exponential bound (4.14) follows from items (iv) and (v) of Proposition 3.7. 
We now set out to construct an expanding sub-solution for the unobstructed LDE (2.1). We introduce the set

$$
\left.\mathcal{D}_{p}=\left\{p=\left(\delta, c, \rho, h_{\infty}, h\right) \in \mathbb{R}^{4} \times C^{2}(\mathbb{R}, \mathbb{R}) \text { for which (i) }\right)_{p} \text { through (iii) } \text { below hold }\right\},
$$

in which the three conditions are specified below.

$(i)_{p}$ Recalling $\delta_{0}$ from Lemma 4.3, we have $0<\delta<\delta_{0}$.

$(\text { ii })_{p}$ We have $\rho>0$ and $0<c<\min _{\zeta \in[0,2 \pi]} c_{\zeta ; \delta}$.

$(\text { iii })_{p}$ We have the inequalities $0 \leq h^{\prime}(\xi) \leq 1$ for all $\xi \in \mathbb{R}$, together with the bound $\left\|h^{\prime \prime}\right\|_{\infty}<\infty$ and the identity $h(\xi)=h(0)=h_{\infty} \geq 0$ for all $\xi \geq 0$.

The variable $\rho \gg 1$ should be seen as the radius of the initial inner core where our sub-solution is close to one and constant, while the function $h$ should be seen as a stretching function that smoothens the transition between an outer region where the sub-solution follows the wave profiles and the inner region where the sub-solution is constant.

For any $p=\left(\delta, c, \rho, h_{\infty}, h\right) \in \mathcal{D}_{p}$, we now introduce the function $u:[0, \infty) \rightarrow \ell^{\infty}\left(\mathbb{Z}^{2} ; \mathbb{R}\right)$ given by

$$
u_{i j}(t)=u_{i j ; p}(t)=\Phi_{\zeta_{i j} ; \delta, h_{\infty}}\left(h\left(\rho+c t-R_{i j}\right)\right),
$$

where the pair $\left(R_{i j}, \zeta_{i j}\right)$ is defined in such a way that

$$
i=R_{i j} \cos \left(\zeta_{i j}\right), \quad j=R_{i j} \sin \left(\zeta_{i j}\right), \quad R_{i j} \geq 0 .
$$

In order to formulate conditions under which $u$ is in fact a sub-solution for (2.1), we define

$$
\begin{aligned}
\mathcal{J}_{i j}^{-}(t) & =\mathcal{J}_{i j ; p}^{-}(t) \\
& =\dot{u}_{i j}(t)-\left[\Delta^{+} u(t)\right]_{i j}-g\left(u_{i j}(t)\right) .
\end{aligned}
$$

Upon introducing the shorthand $z_{i j}=z_{i j}(t)=\rho+c t-R_{i j}$, we may compute

$$
\begin{aligned}
\mathcal{J}_{i j}^{-}(t)=c h^{\prime}\left(z_{i j}\right) & \Phi_{\zeta_{i j} ; \delta, h_{\infty}}^{\prime}\left(h\left(z_{i j}\right)\right) \\
& -\left[\Phi_{\zeta_{i+1, j} ; \delta, h_{\infty}}\left(h\left(z_{i+1, j}\right)\right)+\Phi_{\zeta_{i-1, j} ; \delta, h_{\infty}}\left(h\left(z_{i-1, j}\right)\right)-2 \Phi_{\zeta_{i j} ; \delta, h_{\infty}}\left(h\left(z_{i j}\right)\right)\right] \\
& -\left[\Phi_{\zeta_{i, j+1} ; \delta, h_{\infty}}\left(h\left(z_{i, j+1}\right)\right)+\Phi_{\zeta_{i, j-1} ; \delta, h_{\infty}}\left(h\left(z_{i, j-1}\right)\right)-2 \Phi_{\zeta_{i j} ; \delta, h_{\infty}}\left(h\left(z_{i j}\right)\right)\right] \\
& -g\left(\Phi_{\zeta_{i j} ; \delta, h_{\infty}}\left(h\left(z_{i j}\right)\right)\right) .
\end{aligned}
$$

The wave profile equation (4.2) can be rephrased as

$$
\begin{aligned}
0=-c_{\zeta_{i j} ; \delta} & \Phi_{\zeta_{i j} ; \delta, h_{\infty}}^{\prime}\left(h\left(z_{i j}\right)\right) \\
& +\left[\Phi_{\zeta_{i j} ; \delta, h_{\infty}}\left(h\left(z_{i j}\right)+\cos \zeta_{i j}\right)+\Phi_{\zeta_{i j} ; \delta, h_{\infty}}\left(h\left(z_{i j}\right)-\cos \zeta_{i j}\right)-2 \Phi_{\zeta_{i j} ; \delta, h_{\infty}}\left(h\left(z_{i j}\right)\right)\right] \\
& +\left[\Phi_{\zeta_{i j} ; \delta, h_{\infty}}\left(h\left(z_{i j}\right)+\sin \zeta_{i j}\right)+\Phi_{\zeta_{i j} ; \delta, h_{\infty}}\left(h\left(z_{i j}\right)-\sin \zeta_{i j}\right)-2 \Phi_{\zeta_{i j} ; \delta, h_{\infty}}\left(h\left(z_{i j}\right)\right)\right] \\
& +g_{\delta}^{-}\left(\Phi_{\zeta_{i j} ; \delta, h_{\infty}}\left(h\left(z_{i j}\right)\right)\right) .
\end{aligned}
$$

Upon introducing for any sequence $v: \mathbb{Z}^{2} \rightarrow \mathbb{R}$ the notation

$$
\pi_{i j}^{+} v=\left(v_{i+1, j}, v_{i, j+1}, v_{i-1, j}, v_{i, j-1}, v_{i j}\right) \in \mathbb{R}^{5},
$$

we may write

$$
\mathcal{J}_{i j ; p}^{-}(t)=-\mathcal{H}_{1}\left(\zeta_{i j}, z_{i j}(t) ; p\right)+\mathcal{R}_{2}\left(\pi_{i j}^{+} \zeta, \pi_{i j}^{+} z(t) ; p\right)+\mathcal{R}_{3}\left(\pi_{i j}^{+} \zeta, \pi_{i j}^{+} z(t) ; p\right),
$$


in which we have defined the expressions

$$
\begin{array}{cc}
\mathcal{H}_{1}\left(\zeta_{i j}, z_{i j} ; p\right)= & \left(c_{\zeta_{i j} ; \delta}-c h^{\prime}\left(z_{i j}\right)\right) \Phi_{\zeta_{i j} ; \delta, h_{\infty}}^{\prime}\left(h\left(z_{i j}\right)\right) \\
& -g_{\delta}^{-}\left(\Phi_{\zeta_{i j} ; \delta, h_{\infty}}\left(h\left(z_{i j}\right)\right)\right)+g\left(\Phi_{\zeta_{i j} ; \delta, h_{\infty}}\left(h\left(z_{i j}\right)\right)\right), \\
\mathcal{R}_{2}\left(\pi_{i j}^{+} \zeta, \pi_{i j}^{+} z ; p\right)= & \Phi_{\zeta_{i j} ; \delta, h_{\infty}}\left(h\left(z_{i j}\right)+\cos \zeta_{i j}\right)-\Phi_{\zeta_{i+1, j} ; \delta, h_{\infty}}\left(h\left(z_{i+1, j}\right)\right) \\
& +\Phi_{\zeta_{i j} ; \delta, h_{\infty}}\left(h\left(z_{i j}\right)-\cos \zeta_{i j}\right)-\Phi_{\zeta_{i-1, j} ; \delta, h_{\infty}}\left(h\left(z_{i-1, j}\right)\right), \\
\mathcal{R}_{3}\left(\pi_{i j}^{+} \zeta, \pi_{i j}^{+} z ; p\right)= & \Phi_{\zeta_{i j} ; \delta, h_{\infty}}\left(h\left(z_{i j}\right)+\sin \zeta_{i j}\right)-\Phi_{\zeta_{i, j+1} ; \delta, h_{\infty}}\left(h\left(z_{i, j+1}\right)\right) \\
& +\Phi_{\zeta_{i j} ; \delta, h_{\infty}}\left(h\left(z_{i j}\right)-\sin \zeta_{i j}\right)-\Phi_{\zeta_{i, j-1} ; \delta, h_{\infty}}\left(h\left(z_{i, j-1}\right)\right) .
\end{array}
$$

Roughly speaking, our goal is to show that $\mathcal{H}_{1}$ can be used to dominate $\mathcal{R}_{2}$ and $\mathcal{R}_{3}$. The following series of results will focus on obtaining bounds for the first two of these three expressions, which in view of the symmetry between $\mathcal{R}_{2}$ and $\mathcal{R}_{3}$ will suffice for our purposes.

Lemma 4.5. Suppose that $(\mathrm{hg})_{\S 3}$ and $(H \Phi)$ both hold. Then for any $p \in \mathcal{D}_{p}$, the bound

$$
\mathcal{H}_{1}\left(\zeta_{i j}, z_{i j} ; p\right) \geq\left[\min _{\zeta \in[0,2 \pi]}\left\{c_{\zeta ; \delta}\right\}-c\right] \Phi_{\zeta_{i j} ; \delta, h_{\infty}}^{\prime}\left(h\left(z_{i j}\right)\right)
$$

holds for all pairs $\left(\zeta_{i j}, z_{i j}\right) \in \mathbb{R}^{2}$.

In addition, there exists a constant $\kappa_{3}>0$ such that we have the bound

$$
\mathcal{H}_{1}\left(\zeta_{i j}, z_{i j} ; p\right) \geq \kappa_{3} \delta
$$

for any $p \in \mathcal{D}_{p}$ and $\left(\zeta_{i j}, z_{i j}\right) \in \mathbb{R}^{2}$ for which $\Phi_{\zeta_{i j} ; \delta, h_{\infty}}\left(h\left(z_{i j}\right)\right) \leq 0$.

Proof. This follows directly from $(i i i)_{p}$ together with item (v) of Lemma 3.9.

For convenience, we split the expression $\mathcal{R}_{2}$ into three parts by introducing the expressions

$$
\begin{array}{ccc}
\mathcal{R}_{2, A}\left(\pi_{i j}^{+} \zeta, \pi_{i j}^{+} z ; p\right)= & \Phi_{\zeta_{i j} ; \delta, h_{\infty}}\left(h\left(z_{i j}\right)+\cos \zeta_{i j}\right)-\Phi_{\zeta_{i j} ; \delta, h_{\infty}}\left(h\left(z_{i j}+\cos \zeta_{i j}\right)\right) \\
& +\Phi_{\zeta_{i j} ; \delta, h_{\infty}}\left(h\left(z_{i j}\right)-\cos \zeta_{i j}\right)-\Phi_{\zeta_{i j} ; \delta, h_{\infty}}\left(h\left(z_{i j}-\cos \zeta_{i j}\right)\right), \\
\mathcal{R}_{2, B}\left(\pi_{i j}^{+} \zeta, \pi_{i j}^{+} z ; p\right)= & \Phi_{\zeta_{i j} ; \delta, h_{\infty}}\left(h\left(z_{i j}-\cos \zeta_{i j}\right)\right)-\Phi_{\zeta_{i j} ; \delta, h_{\infty}}\left(h\left(z_{i+1, j}\right)\right) \\
& +\Phi_{\zeta_{i j} ; \delta, h_{\infty}}\left(h\left(z_{i j}+\cos \zeta_{i j}\right)\right)-\Phi_{\zeta_{i j} ; \delta, h_{\infty}}\left(h\left(z_{i-1, j}\right)\right) \\
\mathcal{R}_{2, C}\left(\pi_{i j}^{+} \zeta, \pi_{i j}^{+} z ; p\right)= & \Phi_{\zeta_{i j} ; \delta, h_{\infty}}\left(h\left(z_{i+1, j}\right)\right)-\Phi_{\zeta_{i+1, j} ; \delta, h_{\infty}}\left(h\left(z_{i+1, j}\right)\right) \\
& +\Phi_{\zeta_{i j} ; \delta, h_{\infty}}\left(h\left(z_{i-1, j}\right)\right)-\Phi_{\zeta_{i-1, j} ; \delta, h_{\infty}}\left(h\left(z_{i-1, j}\right)\right) .
\end{array}
$$

This allow us to write

$$
\mathcal{R}_{2}\left(\pi_{i j}^{+} \zeta, \pi_{i j}^{+} z ; p\right)=\mathcal{R}_{2, A}\left(\pi_{i j}^{+} \zeta, \pi_{i j}^{+} z ; p\right)+\mathcal{R}_{2, B}\left(\pi_{i j}^{+} \zeta, \pi_{i j}^{+} z ; p\right)+\mathcal{R}_{2, C}\left(\pi_{i j}^{+} \zeta, \pi_{i j}^{+} z ; p\right)
$$

and we set out to bound each of the components separately.

Lemma 4.6. Suppose that $(\mathrm{hg})_{\S 3}$ and $(H \Phi)$ both hold. Then there exists a constant $K_{4}>1$ such that the following holds true.

Consider any $p \in \mathcal{D}_{p}$, any pair $(i, j) \in \mathbb{Z}^{2}$ and any $t \geq 0$ and suppose that at least one of the two following conditions is satisfied:

(a) For all $z^{\prime} \in \mathbb{R}$ with $\left|z^{\prime}-z_{i j}(t)\right| \leq 1$, we have $h^{\prime}\left(z^{\prime}\right)=1$.

(b) For all $\xi \in \mathbb{R}$ with $\left|\xi-h\left(z_{i j}(t)\right)\right| \leq 2$, we have $\Phi^{\prime \prime}(\xi) \leq 0$. 
Then one has the estimate

$$
\mathcal{R}_{2, A}\left(\pi_{i j}^{+} \zeta, \pi_{i j}^{+} z(t) ; p\right) \leq K_{4} \Phi_{\zeta_{i j} ; \delta, h_{\infty}}^{\prime}\left(h\left(z_{i j}(t)\right)\right)\left\|h^{\prime \prime}\right\|_{\infty} .
$$

Proof. We rewrite $\mathcal{R}_{2, A}$ as the difference

$$
\mathcal{R}_{2, A}\left(\pi_{i j}^{+} \zeta, \pi_{i j}^{+} z ; p\right)=\left.\left.\Phi_{\zeta_{i j} ; \delta, h_{\infty}}\left(h\left(z_{i j}+(\sigma-\tau) \cos \zeta_{i j}\right)+(\sigma+\tau-1) \cos \zeta_{i j}\right)\right|_{\tau=0} ^{\tau=1}\right|_{\sigma=0} ^{\sigma=1}
$$

which allows us to apply the fundamental theorem of calculus to write

$$
\begin{aligned}
& \bar{z}_{i j}(\sigma, \tau)=z_{i j}+(\sigma-\tau) \cos \zeta_{i j}, \\
& \xi_{i j}(\sigma, \tau)=h\left(\bar{z}_{i j}(\sigma, \tau)\right)+(\sigma+\tau-1) \cos \zeta_{i j}
\end{aligned}
$$

and obtain

$$
\begin{array}{r}
\mathcal{R}_{2, A}\left(\pi_{i j}^{+} \zeta, \pi_{i j}^{+} z ; p\right)=\quad \int_{0}^{1} \int_{0}^{1} \Phi^{\prime \prime}\left(\xi_{i j}(\sigma, \tau)\right)\left[1-\left(h^{\prime}\left(\bar{z}_{i j}(\sigma, \tau)\right)\right)^{2}\right] \cos ^{2}\left(\zeta_{i j}\right) d \sigma d \tau \\
-\int_{0}^{1} \int_{0}^{1} \Phi^{\prime}\left(\xi_{i j}(\sigma, \tau)\right) h^{\prime \prime}\left(\bar{z}_{i j}(\sigma, \tau)\right) \cos ^{2}\left(\zeta_{i j}\right) d \sigma d \tau
\end{array}
$$

Note that for all $0 \leq \sigma \leq 1$ and $0 \leq \tau \leq 1$, we have $\left|\bar{z}_{i j}(\sigma, \tau)-z_{i j}\right| \leq 1$ and $\left|\xi_{i j}-h\left(z_{i j}\right)\right| \leq 2$. In particular, if either (a) or (b) holds, the first term in (4.31) is non-positive, which allows us to apply (4.4) and obtain the desired bound.

Lemma 4.7. Suppose that $(\mathrm{hg})_{\S 3}$ and $(H \Phi)$ both hold. Then there exists a constant $K_{5}>1$ such that for any $p \in \mathcal{D}_{p}$, any $(i, j) \in \mathbb{Z}^{2}$ and any $t \geq 0$, we have the bound

$$
\mathcal{R}_{2, B}\left(\pi_{i j}^{+} \zeta, \pi_{i j}^{+} z(t) ; p\right) \leq K_{5} \Phi_{\zeta_{i j} ; \delta, h_{\infty}}^{\prime}\left(h\left(z_{i j}(t)\right)\right) \frac{1}{1+R_{i j}},
$$

which can be sharpened to

$$
\mathcal{R}_{2, B}\left(\pi_{i j}^{+} \zeta, \pi_{i j}^{+} z(t) ; p\right)=0
$$

whenever $z_{i j}(t) \geq 2$.

Proof. The triangle inequality

$$
\left|R_{i j}-R_{i+1, j}\right| \leq 1
$$

gives the bound $\left|z_{i j}-z_{i+1, j}\right| \leq 1$ for all $(i, j) \in \mathbb{Z}^{2}$. An application of the mean value theorem implies

$$
\Phi_{\zeta_{i j} ; \delta, h_{\infty}}\left(h\left(z_{i j}-\cos \zeta_{i j}\right)\right)-\Phi_{\zeta_{i j} ; \delta, h_{\infty}}\left(h\left(z_{i+1, j}\right)\right)=\Phi_{\zeta_{i j} ; \delta}^{\prime}\left(h\left(z_{*}\right)\right) h^{\prime}\left(z_{*}\right)\left[z_{i j}-\cos \zeta_{i j}-z_{i+1, j}\right]
$$

for some $z_{*}$ that has $\left|z_{*}-z_{i j}\right| \leq 2$. The identity (4.33) for $z_{i j} \geq 2$ is now immediate.

Assume for the moment that $(i, j) \neq(0,0)$. Exploiting the elementary observation

$$
\left(R_{i+1, j}-R_{i j}\right)\left(R_{i+1, j}+R_{i j}\right)=R_{i+1, j}^{2}-R_{i j}^{2}=1+2 i,
$$

a little algebra leads to the identity

$$
R_{i+1, j}-R_{i j}-\cos \zeta_{i j}=\frac{1}{2 R_{i j}}\left(1+\frac{(1+2 i)\left(R_{i j}-R_{i+1, j}\right)}{R_{i j}+R_{i+1, j}}\right) .
$$

Again applying (4.34), we find

$$
\begin{aligned}
\left|R_{i+1, j}-R_{i j}-\cos \zeta_{i j}\right| & \leq \frac{1}{2 R_{i j}}\left(1+\frac{|i|+|i+1|}{R_{i j}+R_{i+1, j}}\right) \\
& \leq \frac{1}{R_{i j}}
\end{aligned}
$$


In particular, for any $(i, j) \in \mathbb{Z}^{2}$ we may write

$$
\left|R_{i+1, j}-R_{i j}-\cos \zeta_{i j}\right| \leq \frac{2}{1+R_{i j}} .
$$

Observing the identity $z_{i+1, j}-z_{i j}=R_{i j}-R_{i+1, j}$ and noting the estimate $\left|h\left(z_{*}\right)-h(z)\right| \leq\left|z_{*}-z\right| \leq$ 2 , one can now exploit the uniform bound (4.4) to complete the proof.

Lemma 4.8. Suppose that $(\mathrm{hg})_{\S 3}$ and $(H \Phi)$ both hold and recall the constant $\eta_{*}$ defined in Corollary 4.4. Then for any $p \in \mathcal{D}_{p}$, there exists a constant $K_{6}=K_{6}\left(\delta, h_{\infty}\right)>1$ so that for any pair $(i, j) \in \mathbb{Z}^{2}$ and any $t \geq 0$, we have the bound

$$
\mathcal{R}_{2, C}\left(\pi_{i j}^{+} \zeta, \pi_{i j}^{+} z(t) ; p\right) \leq K_{6} e^{-\eta_{*}\left|h\left(z_{i j}(t)\right)\right|}\left(1+R_{i j}\right)^{-1},
$$

which can be sharpened to the identity

$$
\mathcal{R}_{2, C}\left(\pi_{i j}^{+} \zeta, \pi_{i j}^{+} z(t)\right)=0
$$

whenever $z_{i j}(t) \geq 1$.

Proof. First of all, there exists $C^{\prime}>0$ for which the geometric bound

$$
\left|\zeta_{i+1, j}-\zeta_{i j}\right| \leq C^{\prime}\left(1+R_{i j}\right)^{-1}
$$

holds for all $(i, j) \in \mathbb{Z}^{2}$. We now exploit the mean value theorem to write

$$
\Phi_{\zeta_{i j} ; \delta, h_{\infty}}\left(h\left(z_{i+1, j}\right)\right)-\Phi_{\zeta_{i+1, j} ; \delta, h_{\infty}}\left(h\left(z_{i+1, j}\right)\right)=\partial_{\zeta} \Phi_{\zeta_{*} ; \delta, h_{\infty}}\left(h\left(z_{i+1, j}\right)\right)\left(\zeta_{i+1, j}-\zeta_{i j}\right)
$$

for some $\zeta_{*} \in[0,2 \pi]$. The estimate (4.40) now follows upon combining (4.42) with (4.14).

Finally, the identity (4.41) follows from the fact that $z_{i+1, j} \geq 0$, which implies that $h\left(z_{i+1, j}\right)=h_{\infty}$ and hence

$$
\partial_{\zeta} \Phi_{\zeta_{*} ; \delta, h_{\infty}}\left(h\left(z_{i+1, j}\right)\right)=\partial_{\zeta} \Phi_{\zeta_{*} ; \delta, h_{\infty}}\left(h_{\infty}\right)=\partial_{\zeta} \Phi_{\infty ; h_{\infty}}(\delta)=0 .
$$

We now show how to construct the stretching function $h$, reflecting the specific criteria that arise in the statement of Lemma 4.6. This stretching function is then used to confirm the sub-solution status of the functions (4.16).

Lemma 4.9. For any $\delta_{h}>0$, there exist constants $L=L\left(\delta_{h}\right)>3$ and $h_{\infty}=h_{\infty}\left(\delta_{h}\right)>3$ together with a $C^{2}$-smooth function $h=h\left(\delta_{h}\right): \mathbb{R} \rightarrow \mathbb{R}$ that satisfies the following properties.

(i) We have $h(-L)=0$ and $h(\xi)=h_{\infty}$ for all $\xi \geq 0$.

(ii) For all $\xi \in \mathbb{R}$ we have the bounds $0 \leq h^{\prime}(\xi) \leq 1$ and $-\delta_{h} \leq h^{\prime \prime}(\xi) \leq 0$.

(iii) For all $\xi \leq-L+3$ we have $h^{\prime}(\xi)=1$.

Proof. First of all, write $\ell=L-3$ and consider the polynomial

$$
P(\xi)=\frac{\ell}{2}+\frac{1}{2} \ell^{-3}\left(\xi^{4}+2 \ell \xi^{3}\right) .
$$

It is easy to verify that

$$
P(-\ell)=0, \quad P^{\prime}(-\ell)=1, \quad P^{\prime \prime}(-\ell)=0,
$$

while also

$$
P(0)=\frac{1}{2} \ell, \quad P^{\prime}(0)=0, \quad P^{\prime \prime}(0)=0 .
$$


In addition, for all $-\ell \leq \xi \leq 0$ we have

$$
0 \leq P^{\prime}(\xi)=\ell^{-3} \xi^{2}(2 \xi+3 \ell) \leq 1
$$

and

$$
0 \geq P^{\prime \prime}(\xi)=6 \ell^{-3}\left(\xi^{2}+\ell \xi\right) \geq P^{\prime \prime}\left(-\frac{1}{2} \ell\right)=-\frac{3}{2} \ell^{-1} .
$$

We can hence find the desired function $h$ by picking $\ell \gg 1$ and writing $h(\xi)=3+\xi+\ell$ for $\xi \leq-\ell$, together with $h(\xi)=3+P(\xi)$ for $-\ell \leq \xi \leq 0$ and $h(\xi)=3+\frac{1}{2} \ell$ for $\xi \geq 0$.

Lemma 4.10. Suppose that $(\mathrm{hg})_{\S 3}$ and $(H \Phi)$ both hold and pick any $0<c<\min _{\zeta \in[0,2 \pi] c_{\zeta}}$. Then there exist constants $h_{\infty}=h_{\infty}(c)>0$ and $\delta_{0}=\delta_{0}(c)>0$ together with a $C^{2}$-function $h=h(c)$ : $\mathbb{R} \rightarrow \mathbb{R}$, so that the following holds true.

For any $0<\delta<\delta_{0}$, there exists a constant $\rho=\rho(c, \delta) \gg 1$ such that the function

$$
u_{i j}(t)=\Phi_{\zeta_{i j} ; \delta, h_{\infty}}\left(h\left(\rho+c t-R_{i j}\right)\right)
$$

satisfies the following properties.

(i) For all $t \geq 0$ and $(i, j) \in \mathbb{Z}^{2}$ we have the differential inequality

$$
\dot{u}_{i j}(t) \leq\left[\Delta^{+} u(t)\right]_{i j}(t)+g\left(u_{i j}(t)\right) .
$$

(ii) For all $(i, j) \in \mathbb{Z}^{2}$ and $t \geq 0$ for which $R_{i j} \leq \rho+c t$, we have the inequalities

$$
1-2 \delta<u_{i j}(t)=\Phi_{\infty ; h_{\infty}}(\delta)<1-\delta .
$$

(iii) For all $t \geq 0$ we have the spatial limits

$$
\lim _{|i|+|j| \rightarrow \infty} u_{i j}(t)=-\delta
$$

Proof. First, pick $\kappa^{\prime}>0$ and $\delta_{0}>0$ sufficiently small to ensure that for all $0<\delta<\delta_{0}$ we have

$$
\min _{\zeta \in[0,2 \pi]}\left[c_{\theta ; \delta}-c\right]>\kappa^{\prime}
$$

Now, pick $\delta_{h}$ in such a way that

$$
K_{4} \delta_{h}<\frac{1}{6} \kappa^{\prime}
$$

and recall the function $h$ and constants $L$ and $h_{\infty}$ defined in Lemma 4.9.

Consider now the conditions (a) and (b) in Lemma 4.6. Our choice of the stretching function $h$ implies that we have $h^{\prime}(z)=1$ whenever $z \leq-L+3$, which implies that (a) is satisfied for $z_{i j} \leq-L+2$. For $z_{i j} \geq-L+2$, we note that $h\left(z_{i j}\right) \geq 2$. Since $\Phi_{\theta_{i j} ; \delta, h_{\infty}}^{\prime \prime}(\xi) \leq 0$ for $\xi \geq 0$, we see that (b) is satisfied in this case. In particular, we may conclude that for all $0<\delta<\delta_{0}$ and any $t \geq 0$ we have

$$
\mathcal{R}_{2, A}\left(\pi_{i j}^{+} \zeta, \pi_{i j}^{+} z(t) ; p\right) \leq \frac{1}{6} \kappa^{\prime} \Phi_{\zeta_{i j} ; \delta, h_{\infty}}^{\prime}\left(h\left(z_{i j}(t)\right)\right) \leq \frac{1}{6} \mathcal{H}_{1}\left(\zeta_{i j}, z_{i j}(t) ; p\right) .
$$

Turning our attention to $\mathcal{R}_{2, B}$, we note that Lemma 4.7 implies that

$$
\mathcal{R}_{2, B}\left(\pi_{i j}^{+} \zeta, \pi_{i j}^{+} z(t) ; p\right)=0, \quad z_{i j}(t) \geq 2 .
$$

On the other hand, for $z_{i j} \leq 2$ we see that

$$
R_{i j}=\rho+c t-z_{i j} \geq \rho-2 .
$$


In particular, by choosing $\rho \gg 1$ in such a way that

$$
K_{5}(1+[\rho-2])^{-1} \leq \frac{1}{6} \kappa^{\prime}
$$

we can ensure that for all $0<\delta<\delta_{0}$ we have

$$
\mathcal{R}_{2, B}\left(\pi_{i j}^{+} \zeta, \pi_{i j}^{+} z(t) ; p\right) \leq \frac{1}{6} \mathcal{H}_{1}\left(\zeta_{i j}, z_{i j}(t) ; p\right) .
$$

It remains to consider the term $\mathcal{R}_{2, C}$ in the range $z_{i j} \leq 1$, for which we know $R_{i j} \geq \rho-1$. To this end, fix a value for $0<\delta<\delta_{0}$, recall the setting of Lemma 4.8 and choose $h_{*} \gg 1$ in such a way that

$$
K_{6}\left(\delta, h_{\infty}\right) e^{-\eta_{*} h_{*}} \leq \frac{1}{6} \kappa_{3} \delta
$$

holds, together with

$$
\Phi_{\zeta ; \delta, h_{\infty}}\left(-h_{*}\right) \leq 0, \quad \zeta \in[0,2 \pi]
$$

Lemma 4.5 implies that

$$
\mathcal{R}_{2, C}\left(\pi_{i j}^{+} \zeta, \pi_{i j}^{+} z(t) ; p\right) \leq \frac{1}{6} \mathcal{H}_{1}\left(\zeta_{i j}, z_{i j}(t) ; p\right)
$$

whenever $h\left(z_{i j}(t)\right) \leq-h_{*}$, which is equivalent to $z_{i j}(t) \leq z_{*}$ for some $z_{*} \in \mathbb{R}$. By possibly increasing $\rho \gg 1$, we can now ensure that

$$
K_{6} e^{-\eta_{*}\left|h\left(z_{i j}\right)\right|} \rho^{-1} \leq \frac{1}{6} \kappa^{\prime} \Phi_{\zeta ; \delta, h_{\infty}}^{\prime}\left(h\left(z_{i j}\right)\right)
$$

holds for all $z_{*} \leq z_{i j} \leq 1$. This in fact implies that (4.63) holds for all $z_{i j}(t) \in \mathbb{R}$.

The terms present in $\mathcal{R}_{3}$ can be recovered from the terms in $\mathcal{R}_{2}$ by the symmetry $(i, j, \theta) \mapsto$ $(j, i, \theta+\pi / 2)$. In particular, we can now write

$$
\begin{aligned}
\mathcal{J}_{i j ; p}^{-}(t) & \leq-\mathcal{H}_{1}\left(\zeta_{i j}, z_{i j}(t) ; p\right)+\frac{1}{2} \mathcal{H}_{1}\left(\zeta_{i j}, z_{i j}(t) ; p\right)+\frac{1}{2} \mathcal{H}_{1}\left(\zeta_{i j}, z_{i j}(t) ; p\right) \\
& \leq 0,
\end{aligned}
$$

which establishes (i). The remaining properties (ii) and (iii) follow directly from properties of the profiles $\Phi_{\zeta ; \delta, h_{\infty}}$.

Proof of Proposition 4.1. Pick $\delta_{0}=\delta_{0}(c)$ and $h_{\infty}=h_{\infty}(c)$ from Lemma 4.10 above. For any small $\eta>0$, we can pick $0<\delta<\delta_{0}$ such that $\Phi_{\infty, h_{\infty}}(\delta)=1-\eta$ by continuity and the limit

$$
\lim _{\delta \downarrow 0} \Phi_{\infty, h_{\infty}}(\delta)=1 .
$$

The result now follows directly from Lemma 4.10.

\section{$5 \quad$ Large Disturbances}

In this section we show how large but localized disturbances to planar travelling waves can be controlled by suitably constructed sub and super-solutions. In particular, we show that such disturbances eventually die out, showing that the planar waves are extremely robust.

Our focus will be on planar waves that travel in the rational direction $\left(\sigma_{h}, \sigma_{v}\right) \in \mathbb{Z}^{2} \backslash\{0,0\}$. To ease our notation, we introduce a new coordinate system that reflects the geometry of the wave. In particular, we write

$$
\begin{aligned}
& n=i \sigma_{h}+j \sigma_{v}, \\
& l=i \sigma_{v}-j \sigma_{h} .
\end{aligned}
$$


The first of these coordinates represents the direction parallel to the propagation of the wave, while the second coordinate represents the direction perpendicular to wave motion. In the sequel we often refer to $n$ as the wave coordinate and $l$ as the transverse coordinate.

We emphasize that we always have $(n, l) \in \mathbb{Z}^{2}$ due to our assumption that $\left(\sigma_{h}, \sigma_{v}\right) \in \mathbb{Z}^{2}$. However, the inverse of the transformation (5.1) is given by

$$
\begin{aligned}
i & =\left[\sigma_{h}^{2}+\sigma_{v}^{2}\right]^{-1}\left(n \sigma_{h}+l \sigma_{v}\right), \\
j & =\left[\sigma_{h}^{2}+\sigma_{v}^{2}\right]^{-1}\left(n \sigma_{v}-l \sigma_{h}\right),
\end{aligned}
$$

which means that the pairs $(n, l)$ in the range of the transformation $(5.1)$ represent only a sublattice of $\mathbb{Z}^{2}$. We choose to ignore this issue in the current paper, simply taking $(n, l) \in \mathbb{Z}^{2}$. One can think of this choice as simply solving a number of independent systems simultaneously.

Rewriting the homogeneous LDE (2.1) in terms of our new coordinates, we obtain the system

$$
\dot{u}_{n l}(t)=\left[\Delta^{\times} u(t)\right]_{n l}+g\left(u_{n l}(t)\right), \quad(n, l) \in \mathbb{Z}^{2} .
$$

Here we have introduced the notation

$$
\left[\Delta^{\times} u\right]_{n l}=\sum_{\left(n^{\prime}, l^{\prime}\right) \in \mathcal{N}_{\mathbb{Z}^{2}}^{\times}(n, l)}\left[u_{n^{\prime} l^{\prime}}-u_{i j}\right]
$$

for any $u \in \ell^{\infty}\left(\mathbb{Z}^{2} ; \mathbb{R}\right)$, in which the neighbour set

$$
\mathcal{N}_{\mathbb{Z}^{2}}^{\times}(n, l)=\left\{\left(n+\sigma_{h}, l+\sigma_{v}\right),\left(n+\sigma_{v}, l-\sigma_{h}\right),\left(n-\sigma_{h}, l-\sigma_{v}\right),\left(n-\sigma_{v}, l+\sigma_{h}\right)\right\} \subset \mathbb{Z}^{2}
$$

encodes the geometry of the new coordinate system.

The planar travelling wave solutions (3.9) can now be written as

$$
u_{n l}(t)=\Phi(n+c t) .
$$

The main result of this section constructs sub and super-solutions for (5.3) that converge to shifted versions of (5.6). Our version is rather technical as we intend it to be strong enough to allow the effects of the obstacle to be included later on. The properties (vi) - (viii) together with the fact that we do not prescribe a specific choice for $z$ should be seen in this light. On the other hand, the algebraic decay properties imposed on $z$ and stated in (iii) can be seen as direct consequences of the discrete nature of the lattice.

Proposition 5.1. Consider any angle $\zeta_{*}$ with $\tan \zeta_{*} \in \mathbb{Q}$ and suppose that $(H g)$ and $(H S)_{\zeta_{*}}$ both hold. Pick $\left(\sigma_{h}, \sigma_{v}\right) \in \mathbb{Z}^{2} \backslash\{(0,0)\}$ with the property that

$$
\sqrt{\sigma_{h}^{2}+\sigma_{v}^{2}}\left(\cos \zeta_{*}, \sin \zeta_{*}\right)=\left(\sigma_{h}, \sigma_{v}\right), \quad \operatorname{gcd}\left(\sigma_{h}, \sigma_{v}\right)=1
$$

and suppose that $(h \Phi)_{\S 3}$ holds for this pair $\left(\sigma_{h}, \sigma_{v}\right)$ with $c>0$.

Then there exist constants

$$
\delta_{\epsilon}>0, \quad \eta_{z}>0, \quad K_{Z}>1, \quad K_{\mathcal{N}}>1, \quad \eta_{\mathcal{N}}>0
$$

such that for any triplet $\left(\epsilon_{1}, \epsilon_{2}, \epsilon_{3}\right)$ that has

$$
0<2 \epsilon_{2}<\epsilon_{1} \leq \delta_{\epsilon}, \quad 0<\epsilon_{3} \leq \epsilon_{1}
$$

and any pair $\Omega_{\perp}>0, \Omega_{\text {phase }}>0$, there exists a function $\theta:[0, \infty) \rightarrow \ell^{\infty}(\mathbb{Z} ; \mathbb{R})$ so that the following holds true.

Consider any phase shift $\vartheta \in \mathbb{R}$ and any function $z:[0, \infty) \rightarrow \mathbb{R}$ that satisfies the conditions 
$(i)_{z}$ We have $z^{\prime}(t) \geq-\eta_{z} z(t)$ for all $t \geq 0$.

$(\text { ii })_{z}$ We have $0<z(t) \leq z(0)=\epsilon_{1}$ for all $t \geq 0$.

(iii) $)_{z}$ We have $z(t) \geq \epsilon_{3}(1+t)^{-3 / 2}$.

There exist functions $W^{ \pm}:[0, \infty) \rightarrow \ell^{\infty}\left(\mathbb{Z}^{2} ; \mathbb{R}\right)$ and $\xi^{ \pm}:[0, \infty) \rightarrow \ell^{\infty}\left(\mathbb{Z}^{2} ; \mathbb{R}\right)$ that satisfy the following properties.

(i) The quantities

$$
\begin{aligned}
& \mathcal{J}_{n l}^{-}(t)=\dot{W}_{n l}^{-}(t)-\left[\Delta^{\times} W^{-}(t)\right]_{n l}-g\left(W_{n l}^{-}(t)\right), \\
& \mathcal{J}_{n l}^{+}(t)=\dot{W}_{n l}^{+}(t)-\left[\Delta^{\times} W^{+}(t)\right]_{n l}-g\left(W_{n l}^{+}(t)\right),
\end{aligned}
$$

satisfy the bounds

$$
\begin{aligned}
& \mathcal{J}_{n l}^{-}(t) \leq-\frac{1}{2} \eta_{z} z(t), \\
& \mathcal{J}_{n l}^{+}(t) \geq+\frac{1}{2} \eta_{z} z(t),
\end{aligned}
$$

for all $t \geq 0$ and $(n, l) \in \mathbb{Z}^{2}$.

(ii) For $|l| \leq \Omega_{\perp}$, we have

$$
W_{n l}^{-}(0) \leq \Phi\left(n+c t+\vartheta-\Omega_{\text {phase }}\right), \quad W_{n l}^{+}(0) \geq \Phi\left(n+c t+\vartheta+\Omega_{\text {phase }}\right) .
$$

(iii) For every $t \geq 0$ and every $(n, l) \in \mathbb{Z}^{2}$, we have the bounds

$$
\begin{aligned}
& \Phi\left(\xi_{n l}^{-}(t)\right)-\epsilon_{2}(1+t)^{-1 / 2} \leq W_{n l}^{-}(t)+z(t) \leq \Phi\left(\xi_{n l}^{-}(t)\right)+\epsilon_{2}(1+t)^{-1 / 2} \\
& \Phi\left(\xi_{n l}^{+}(t)\right)-\epsilon_{2}(1+t)^{-1 / 2} \leq W_{n l}^{+}(t)-z(t) \leq \Phi\left(\xi_{n l}^{+}(t)\right)+\epsilon_{2}(1+t)^{-1 / 2}
\end{aligned}
$$

(iv) We have $\theta_{l}(t) \geq 0$ for all $t \geq 0$ and $l \in \mathbb{Z}$, together with the uniform limit

$$
\lim _{t \rightarrow \infty}\left[\sup _{l \in \mathbb{Z}} \theta_{l}(t)\right]=0
$$

(v) Introducing the function

$$
Z(t)=K_{Z} \int_{0}^{t} z\left(t^{\prime}\right) d t^{\prime}
$$

we have the identities

$$
\begin{aligned}
& \xi_{n l}^{-}(t)=n+c t+\vartheta-\theta_{l}(t)-Z(t), \\
& \xi_{n l}^{+}(t)=n+c t+\vartheta+\theta_{l}(t)+Z(t) .
\end{aligned}
$$

(vi) Consider any bounded set $S \subset \mathbb{Z}^{2}$. Upon writing

$$
\operatorname{diam}(S)=\sup _{(n, l) \in S,\left(n^{\prime}, l^{\prime}\right) \in S}\left[\left|n-n^{\prime}\right|+\left|l-l^{\prime}\right|\right],
$$

we have the uniform bounds

$$
\begin{aligned}
& \max _{(n, l) \in S} \xi_{n l}^{-}(t)-\min _{(n, l) \in S} \xi_{n l}^{-}(t) \leq 1+\operatorname{diam}(S), \\
& \max _{(n, l) \in S} \xi_{n l}^{+}(t)-\min _{(n, l) \in S} \xi_{n l}^{+}(t) \leq 1+\operatorname{diam}(S),
\end{aligned}
$$

for every $t \geq 0$. 
(vii) For any $(n, l) \in \mathbb{Z}^{2}$ with $|l| \leq \Omega_{\perp}$ and $t \geq 0$, we have

$$
\dot{\xi}_{n l}^{ \pm}(t) \geq \frac{c}{2} .
$$

(viii) For any pairs $(n, l) \in \mathbb{Z}^{2}$ and $\left(n^{\prime}, l^{\prime}\right) \in \mathcal{N}_{\mathbb{Z}^{2}}^{\times}(n, l)$, we have the bounds

$$
\left|W_{n l}^{ \pm}(t)-W_{n^{\prime} l^{\prime}}^{ \pm}(t)\right| \leq K_{\mathcal{N}} e^{-\eta_{\mathcal{N}}}\left|\xi_{n l}^{ \pm}(t)\right| .
$$

Notice that a direct consequence of (iii), (iv) and (v) is that for all $(n, l) \in \mathbb{Z}^{2}$, we have

$$
W_{n l}^{-}(0) \leq \Phi(n+c t+\vartheta)-\frac{1}{2} \epsilon_{1}, \quad W_{n l}^{+}(0) \geq \Phi(n+c t+\vartheta)+\frac{1}{2} \epsilon_{1},
$$

which shows that we can indeed interpret the result above as a mechanism for turning small global additive perturbations into small phase shifts, as customary in one-dimensional results of this nature. The extra feature in two dimensions is that we can also include large localized phase shifts in the initial perturbation.

In order to assist the reader in interpreting the result above, we conclude this subsection by using it to establish the nonlinear stability of the travelling wave (5.6), as stated in Theorem 2.2. As a preparation, we construct a template function $z_{\text {hom }}$ that satisfies the requirements $(i)_{z}$ through $(\text { iii })_{z}$.

Lemma 5.2. Fix any $0<\eta_{z}<1$. Then there exists constants $\mathcal{I}_{\text {hom }}=\mathcal{I}_{\text {hom }}\left(\eta_{z}\right)>1$ and $\kappa_{\text {hom }}=$ $\kappa_{\mathrm{hom}}\left(\eta_{z}\right)>0$ together with a $C^{1}$-smooth function $z_{\mathrm{hom}}:[0, \infty) \rightarrow \mathbb{R}$ that satisfies the following properties.

(i) We have $z_{\mathrm{hom}}^{\prime}(t) \geq-\eta_{z} z_{\mathrm{hom}}(t)$ for all $t \geq 0$.

(ii) We have $\kappa_{\mathrm{hom}}(1+t)^{-3 / 2} \leq z_{\mathrm{hom}}(t) \leq z_{\mathrm{hom}}(0)=1$ for all $t \geq 0$.

(iii) We have $\int_{0}^{\infty} z_{\mathrm{hom}}(t) d t<\mathcal{I}_{\mathrm{hom}}$.

Proof. For $0 \leq t \leq \frac{3}{2} \eta_{z}^{-1}-1$ we write

$$
z_{\mathrm{hom}}(t)=e^{-\eta_{z} t}
$$

while for $t \geq \frac{3}{2} \eta_{z}^{-1}-1$ we write

$$
z_{\mathrm{hom}}(t)=\eta_{z}^{-3 / 2}\left(\frac{3}{2}\right)^{3 / 2} e^{\eta_{z}-\frac{3}{2}}(1+t)^{-3 / 2} .
$$

One can readily verify that $z_{\text {hom }}$ is $C^{1}$-smooth and that (i) and (ii) are satisfied. Property (iii) follows from the identity

$$
\int_{0}^{\infty} z_{\mathrm{hom}}(t) d t=\eta_{z}^{-1}\left[2 e^{\eta_{z}-\frac{3}{2}}+1\right] .
$$

Proof of Theorem 2.2. Pick any $\delta_{*}>0$. We restrict ourselves here to showing that

$$
\liminf _{t \rightarrow \infty} \inf _{(n, l) \in \mathbb{Z}^{2}}\left[U_{n l}(t)-\Phi(n+c t)\right] \geq-\delta_{*},
$$

noting that the companion bound

$$
\limsup _{t \rightarrow \infty} \sup _{(n, l) \in \mathbb{Z}^{2}}\left[U_{n l}(t)-\Phi(n+c t)\right] \leq+\delta_{*},
$$


can be obtained in a similar fashion.

Pick $\epsilon_{1}>0$ in such a way that

$$
\epsilon_{1} K_{Z} \mathcal{I}_{\text {hom }}\left\|\Phi^{\prime}\right\|_{\infty} \leq \delta_{*}
$$

and write $\epsilon_{2}=\frac{1}{2} \epsilon_{1}, \epsilon_{3}=\epsilon_{1} \kappa_{\text {hom }}, \vartheta=0$ and $z(t)=\epsilon_{1} z_{\text {hom }}(t)$. There exists a finite set $S_{\epsilon_{2}} \subset \mathbb{Z}^{2}$ for which

$$
\left|U_{n l}(0)-\Phi(n)\right| \leq \epsilon_{2}
$$

holds for all $(n, l) \in \mathbb{Z}^{2} \backslash S_{\epsilon_{2}}$. In particular, by picking $\Omega_{\perp}$ and $\Omega_{\text {phase }}$ appropriately, we can ensure that

$$
W_{n l}^{-}(0) \leq U_{n l}(0)
$$

holds for all $(n, l) \in \mathbb{Z}^{2}$. Since

$$
\lim _{t \rightarrow \infty} \sup _{(n, l) \in \mathbb{Z}^{2}}\left[W_{n l}^{-}(t)-\Phi(n+c t-Z(t))\right]=0,
$$

while also

$$
|\Phi(n+c t)-\Phi(n+c t-Z(t))| \leq\left\|\Phi^{\prime}\right\|_{\infty}|Z(t)| \leq \epsilon_{1}\left\|\Phi^{\prime}\right\|_{\infty} K_{Z} \mathcal{I}_{\mathrm{hom}} \leq \delta_{*},
$$

the comparison principle directly implies (5.25).

\section{$5.1 \quad$ Notation}

In this subsection we set up the notation that will be used throughout $\S 5$. In addition, we perform some preliminary computations that will aid us in the construction of the sub and super-solutions described in Proposition 5.1.

First of all, we introduce for any $u \in \ell^{\infty}\left(\mathbb{Z}^{2} ; \mathbb{R}\right)$ and any $(n, l) \in \mathbb{Z}^{2}$, the vector

$$
\pi_{n l}^{\times} u=\left(u_{n+\sigma_{h}, l+\sigma_{v}}, u_{n+\sigma_{v}, l-\sigma_{h}}, u_{n-\sigma_{h}, l-\sigma_{v}}, u_{n-\sigma_{v}, l+\sigma_{h}}, u_{n l}\right) \in \mathbb{R}^{5},
$$

which can be seen as evaluating $u$ on a stencil of grid points that consists of $(n, l)$ and its nearest neighbours $\mathcal{N}_{\mathbb{Z}^{2}}^{\times}(n, l)$.

Upon introducing the vector

$$
L^{\times}=(1,1,1,1,-4) \in \mathbb{R}^{5},
$$

we can now rewrite (5.3) in the form

$$
\dot{u}_{n l}(t)=L^{\times} \pi_{n l}^{\times} u(t)+g\left(u_{n l}(t)\right) .
$$

To avoid clutter, we also introduce the operator

$$
\pi^{\times}: \ell^{\infty}\left(\mathbb{Z}^{2} ; \mathbb{R}\right) \rightarrow \ell^{\infty}\left(\mathbb{Z}^{2}, \mathbb{R}^{5}\right)
$$

that acts as

$$
\left[\pi^{\times} u\right]_{n l}=\pi_{n l}^{\times} u .
$$

This allows us to restate (5.3) as

$$
\dot{u}(t)=L^{\times} \pi^{\times} u(t)+g(u(t)),
$$

in which the nonlinearity $g$ is interpreted to act componentwise. We will refer to equations such as (5.37), where the dependence on $(n, l)$ has been dropped, as equations in global form. Similarly, equations such as (5.34) are called equations in local form. 


\section{Summation Convention}

Throughout the sequel we will use greek indices

$$
\mu, \mu^{\prime}, \mu^{\prime \prime} \in\{1,2,3,4,5\}, \quad \nu, \nu^{\prime}, \nu^{\prime \prime} \in\{1,2,3,4,5\}
$$

with the following summation convention. The indices $\mu, \mu^{\prime}, \mu^{\prime \prime}$ will only appear on the right side of identities and any term involving $n \geq 1$ distinct greek indices needs to be summed over all $5^{n}$ combinations of these indices. On the other hand, the indices $\nu, \nu^{\prime}, \nu^{\prime \prime}$ may appear on both sides of an identity and do not require a summation.

We refer to individual components of $\pi^{\times}$and $L^{\times}$by writing

$$
\pi_{n l}^{\times}=\left(\pi_{n l ; 1}^{\times}, \ldots, \pi_{n l ; 5}^{\times}\right), \quad L^{\times}=\left(L_{1}^{\times}, \ldots, L_{5}^{\times}\right),
$$

together with

$$
\pi^{\times}=\left(\pi_{; 1}^{\times}, \ldots, \pi_{; 5}^{\times}\right) .
$$

In particular, the local form (5.34) can be written as

$$
\dot{u}_{n l}(t)=L_{\mu}^{\times} \pi_{n l ; \mu}^{\times} u(t)+g\left(u_{n l}(t)\right),
$$

while the global form (5.37) can be written as

$$
\dot{u}(t)=L_{\mu}^{\times} \pi_{; \mu}^{\times} u(t)+g(u(t)) .
$$

Let us now fix a speed $c \in \mathbb{R}$ together with a $C^{1}$-smooth function $\theta:[0, \infty) \rightarrow \ell^{\infty}(\mathbb{Z} ; \mathbb{R})$ and a $C^{1}$-smooth function $Z:[0, \infty) \rightarrow \mathbb{R}$. In what follows, a crucial role will be played by the related quantities

$$
\xi_{n l}(t):=n+c t-\theta_{l}(t)-Z(t), \quad(n, l) \in \mathbb{Z}^{2}, \quad t \geq 0 .
$$

In particular, let us consider any $C^{2}$-smooth function $h: \mathbb{R} \rightarrow \mathbb{R}$ for which $h, h^{\prime}$ and $h^{\prime \prime}$ are all bounded. Upon introducing the notation

$$
\mathrm{J}_{n l}(h ; t)=h\left(\xi_{n l}(t)\right)
$$

and writing

$$
\mathrm{J}(h ; t) \in \ell^{\infty}\left(\mathbb{Z} ; \mathbb{R}^{2}\right)
$$

for the sequence that has

$$
[\mathrm{J}(h ; t)]_{n l}=\mathrm{J}_{n l}(h ; t),
$$

we observe that the map $t \mapsto \mathrm{J}(h ; t)$ is a $C^{1}$-smooth map from $[0, \infty)$ into $\ell^{\infty}\left(\mathbb{Z} ; \mathbb{R}^{2}\right)$. In particular, by evaluating at $\xi_{n l}(t)$ the scalar function $h$ has been transformed into a smooth sequence-valued function. We lose an order of smoothness here because of the uniform continuity requirements arising from the $\ell^{\infty}$ norm.

Let us now consider two functions

$$
p, q \in C^{1}\left([0, \infty), \ell^{\infty}(\mathbb{Z} ; \mathbb{R})\right) .
$$

We introduce the notation

$$
\mathrm{J} n l(q, h ; t)=q_{l}(t) h\left(\xi_{n l}(t)\right), \quad \mathrm{J} n l(p, q, h ; t)=p_{l}(t) q_{l}(t) h\left(\xi_{n l}(t)\right)
$$

and write

$$
\mathrm{J}(q, h ; t) \in \ell^{\infty}\left(\mathbb{Z} ; \mathbb{R}^{2}\right) \quad \mathrm{J}(p, q, h ; t) \in \ell^{\infty}\left(\mathbb{Z} ; \mathbb{R}^{2}\right)
$$

for the sequences that have

$$
[\mathrm{J}(q, h ; t)]_{n l}=\mathrm{J}_{n l}(q, h ; t), \quad[\mathrm{J}(p, q, h ; t)]_{n l}=\mathrm{J}_{n l}(p, q, h ; t) .
$$


As before, the maps $t \mapsto \mathrm{J}(q, h ; t)$ and $t \mapsto \mathrm{J}(p, q, h ; t)$ are $C^{1}$-smooth maps from $[0, \infty)$ into $\ell^{\infty}\left(\mathbb{Z} ; \mathbb{R}^{2}\right)$.

Turning our attention to sequences $\theta \in \ell^{\infty}(\mathbb{Z} ; \mathbb{R})$, we need to introduce a number of difference operators. To this end, we define the shifts

$$
\left(\sigma_{1}, \ldots, \sigma_{5}\right)=\left(\sigma_{v},-\sigma_{h},-\sigma_{v}, \sigma_{h}, 0\right)
$$

and introduce the notation

$$
\pi_{l}^{\diamond} \theta=\left(\pi_{l ; 1}^{\diamond} \theta, \ldots, \pi_{l ; 5}^{\diamond} \theta\right) \in \mathbb{R}^{5}
$$

for first difference operators $\pi_{l ; \nu}^{\diamond}$ that act as

$$
\pi_{l ; \nu}^{\diamond} \theta=\theta_{l+\sigma_{\nu}}-\theta_{l}, \quad 1 \leq \nu \leq 5 .
$$

In global form, we write

$$
\pi^{\diamond} \theta=\left\{\pi_{l}^{\diamond} \theta\right\}_{l \in \mathbb{Z}} \in \ell^{\infty}\left(\mathbb{Z} ; \mathbb{R}^{5}\right)
$$

and refer to the individual components as

$$
\pi_{; \nu}^{\diamond} \theta=\left\{\pi_{l ; \nu}^{\diamond} \theta\right\}_{l \in \mathbb{Z}} \in \ell^{\infty}(\mathbb{Z} ; \mathbb{R}) .
$$

In a similar fashion, we introduce the notation

$$
\pi_{l}^{\diamond \diamond} \theta=\left(\pi_{l ; \nu \nu^{\prime}}^{\diamond \diamond} \theta\right)_{\left(\nu, \nu^{\prime}\right) \in\{1, \ldots 5\}^{2}} \in \mathbb{R}^{5 \times 5},
$$

with second difference operators that act as

$$
\pi_{l ; \nu \nu^{\prime}}^{\diamond \diamond}=\left(\theta_{l+\sigma_{\nu}+\sigma_{\nu}^{\prime}}-\theta_{l+\sigma_{\nu}^{\prime}}\right)-\left(\theta_{l+\sigma_{\nu}}-\theta_{l}\right) .
$$

In other words, we have

$$
\pi_{l ; \nu \nu^{\prime}}^{\diamond \diamond}=\pi_{l ; \nu^{\prime}}^{\diamond} \pi_{; \nu}^{\diamond} \theta
$$

In global form, we write

$$
\pi^{\diamond \diamond} \theta=\left\{\pi_{l}^{\diamond \diamond} \theta\right\}_{l \in \mathbb{Z}} \in \ell^{\infty}\left(\mathbb{Z} ; \mathbb{R}^{5 \times 5}\right), \quad \pi_{; \nu \nu^{\prime}}^{\diamond \diamond} \theta=\left\{\pi_{l ; \nu \nu^{\prime}}^{\diamond \diamond} \theta\right\}_{l \in \mathbb{Z}} \in \ell^{\infty}(\mathbb{Z} ; \mathbb{R}) .
$$

Naturally, this allows us to define third differences

$$
\pi^{\diamond \diamond \diamond} \theta \in \ell^{\infty}\left(\mathbb{Z} ; \mathbb{R}^{5 \times 5 \times 5}\right)
$$

by means of the components

$$
\pi_{l ; \nu \nu^{\prime} \nu^{\prime \prime}}^{\diamond \diamond \diamond} \theta=\pi_{l ; \nu^{\prime \prime}}^{\diamond} \pi_{; \nu \nu^{\prime}}^{\diamond} \theta=\pi_{l ; \nu^{\prime \prime}}^{\diamond} \pi_{; \nu^{\prime}}^{\diamond} \pi_{; \nu}^{\diamond} \theta .
$$

We also need to consider a second transformation of the function $h$. To this end, we define the five constants

$$
\left(\tau_{1}, \ldots, \tau_{5}\right)=\left(\sigma_{h}, \sigma_{v},-\sigma_{h},-\sigma_{v}, 0\right)
$$

and write $\tau h \in C^{2}\left(\mathbb{R}, \mathbb{R}^{5}\right)$ for the function that has

$$
[\tau h](\xi)=\left(h\left(\xi+\tau_{1}\right), \ldots, h\left(\xi+\tau_{5}\right)\right) \in \mathbb{R}^{5} .
$$

Abusing notation, we often write

$$
[\tau h](\xi)=\left(\left[\tau_{1} h\right](\xi), \ldots,\left[\tau_{5} h\right](\xi)\right)
$$

for the five components of $\tau h$. We note that the pairing of the constants $\sigma_{\mu}$ and $\tau_{\mu}$ comes directly from the form of the neighbour set $\mathcal{N}_{\mathbb{Z}^{2}}^{\times}$defined in (5.5). 
For any $(n, l) \in \mathbb{Z}^{2}$ and $t \geq 0$, we write

$$
\mathrm{J}_{n l}(L ; t)=L^{\times}+\left(0,0,0,0, g^{\prime}\left(\Phi\left(\xi_{n l}(t)\right)\right)\right) \in \mathbb{R}^{5} .
$$

In particular, for any sequence $u \in \ell^{\infty}\left(\mathbb{Z}^{2} ; \mathbb{R}\right)$ we have

$$
\mathrm{J}_{n l}(L ; t) \pi_{n l}^{\times} u=L_{\mu}^{\times} \pi_{n l ; \mu}^{\times} u+g^{\prime}\left(\Phi\left(\xi_{n l}(t)\right)\right) u_{n l} .
$$

In global form, we shorten this to

$$
\mathrm{J}(L ; t) \pi^{\times} u=L_{\mu}^{\times} \pi_{; \mu}^{\times} u+g^{\prime}(\Phi(\xi(t))) u .
$$

For convenience, we often use the shorthand

$$
\begin{aligned}
\mathrm{J}_{n l}(L \tau h ; t) & =\mathrm{J}_{n l}(L ; t)_{\mathrm{J} n l}(\tau h ; t) \\
& =L_{\mu}^{\times}\left[\tau_{\mu} h\right]\left(\xi_{n l}(t)\right)+g^{\prime}\left(\Phi\left(\xi_{n l}(t)\right)\right) h\left(\xi_{n l}(t)\right) \\
& =L_{\mu}^{\times} h\left(\xi_{n l}(t)+\tau_{\mu}\right)+g^{\prime}\left(\Phi\left(\xi_{n l}(t)\right)\right) h\left(\xi_{n l}(t)\right),
\end{aligned}
$$

together with

$$
\begin{aligned}
\mathrm{J}_{n l}(q, L \tau h ; t) & =q_{l}(t) \mathrm{J}_{n l}(L ; t) \mathrm{J}_{n l}(\tau h ; t) \\
& =q_{l}(t) \mathrm{J}_{n l}(L \tau h ; t), \\
\mathrm{J}_{n l}(p, q, L \tau h ; t) & =p_{l}(t) q_{l}(t) \mathrm{J}_{n l}(L ; t) \mathrm{J}_{n l}(\tau h ; t) \\
& =p_{l}(t) q_{l}(t) \mathrm{J}_{n l}(L \tau h ; t) .
\end{aligned}
$$

In global form, we write

$$
\begin{array}{ll}
\mathrm{J}(L \tau h ; t) & =\mathrm{J}(L ; t) \mathrm{J}(\tau h ; t), \\
\mathrm{J}(q, L \tau h ; t) & =q(t) \mathrm{J}(L \tau h ; t), \\
\mathrm{J}(p, q, L \tau h ; t) & =p(t) q(t) \mathrm{J}(L \tau h ; t) .
\end{array}
$$

\subsection{Preliminary Computations}

In this subsection we set out to derive a number of tractable expressions for the quantities

$$
\mathrm{J}(L ; t) \pi^{\times} \mathrm{J}(h ; t), \quad \mathrm{J}(L ; t) \pi^{\times} \mathrm{J}(q, h ; t), \quad \mathrm{J}(L ; t) \pi^{\times} \mathrm{J}(p, q, h ; t),
$$

since these play a crucial role in the verification of the relevant differential inequalities for our subsolution. For use in the sequel when discussing obstacle problems, we also consider the quantities

$$
\left[\pi_{; \nu}^{\times}-\pi_{; 5}^{\times}\right] \mathrm{J}(h ; t), \quad\left[\pi_{; \nu}^{\times}-\pi_{; 5}^{\times}\right] \mathrm{J}(q, h ; t), \quad\left[\pi_{; \nu}^{\times}-\pi_{; 5}^{\times}\right] \mathrm{J}(p, q, h ; t) .
$$

As in $\S 5.1$, the function $h: \mathbb{R} \rightarrow \mathbb{R}$ is assumed to be $C^{2}$-smooth with uniform bounds for $h, h^{\prime}$ and $h^{\prime \prime}$, while $p$ and $q$ are assumed to be two $C^{1}$-smooth functions mapping $[0, \infty)$ into $\ell^{\infty}(\mathbb{Z} ; \mathbb{R})$.

We start by writing

$$
\begin{aligned}
\pi_{n l ; \nu}^{\times} \mathrm{J}(h ; t) & =\mathrm{J} n l\left(\tau_{\nu} h ; t\right)+\mathcal{M}_{h, 1 ; \nu}\left(\xi_{n l}(t), \pi_{l ; \nu}^{\diamond} \theta(t)\right) \\
& =\mathrm{J} n l\left(\tau_{\nu} h ; t\right)-\pi_{l ; \nu}^{\diamond} \theta(t) \mathrm{J}_{n l}\left(\tau_{\nu} h^{\prime} ; t\right)+\mathcal{M}_{h, 2 ; \nu}\left(\xi_{n l}(t), \pi_{l ; \nu}^{\diamond} \theta(t)\right),
\end{aligned}
$$

which should be seen as implicit definitions for the expressions $\mathcal{M}_{h, 1 ; \nu}$ and $\mathcal{M}_{h, 2 ; \nu}$. The mean value theorem implies the identities

$$
\begin{aligned}
& \mathcal{M}_{h, 1 ; \nu}\left(\xi_{n l}, \pi_{l ; \nu}^{\diamond} \theta\right)=h^{\prime}\left(\xi_{n l}+\tau_{\nu}+\vartheta_{1}\left[\theta_{l}-\theta_{l+\sigma_{\nu}}\right]\right)\left[\theta_{l}-\theta_{l+\sigma_{\nu}}\right], \\
& \mathcal{M}_{h, 2 ; \nu}\left(\xi_{n l}, \pi_{l ; \nu}^{\diamond} \theta\right)=\frac{1}{2} h^{\prime \prime}\left(\xi_{n l}+\tau_{\nu}+\vartheta_{2}\left[\theta_{l}-\theta_{l+\sigma_{\nu}}\right]\right)\left[\theta_{l}-\theta_{l+\sigma_{\nu}}\right]^{2},
\end{aligned}
$$


for some pair $0<\vartheta_{1}<1$ and $0<\vartheta_{2}<1$ that depends on $\xi_{n l} \in \mathbb{R}$ and $\pi_{l ; \nu}^{\diamond} \theta \in \mathbb{R}^{5}$. In particular, the uniform bounds on $h^{\prime}$ and $h^{\prime \prime}$ imply that there exists $C>0$ such that

$$
\begin{aligned}
& \mathcal{M}_{h, 1 ; \nu}\left(\xi_{n l}, \pi_{l ; \nu}^{\diamond} \theta\right) \leq C\left|\pi_{l ; \nu}^{\diamond} \theta\right|, \\
& \mathcal{M}_{h, 2 ; \nu}\left(\xi_{n l}, \pi_{l ; \nu}^{\diamond} \theta\right) \leq C\left|\pi_{l ; \nu}^{\diamond} \theta\right|^{2},
\end{aligned}
$$

for any $\xi_{n l} \in \mathbb{R}$, any $\pi_{l ; \nu}^{\diamond} \theta \in \mathbb{R}$ and any integer $1 \leq \nu \leq 5$. For convenience, for any $t \geq 0$ we introduce the global form expressions

$$
\mathcal{N}_{h, 1 ; \nu}\left(\pi_{; \nu}^{\diamond} \theta ; t\right) \in \ell^{\infty}\left(\mathbb{Z}^{2} ; \mathbb{R}\right), \quad \mathcal{N}_{h, 2 ; \nu}\left(\pi_{; \nu}^{\diamond} \theta ; t\right) \in \ell^{\infty}\left(\mathbb{Z}^{2} ; \mathbb{R}\right)
$$

that are given by

$$
\left[\mathcal{N}_{h, 1 ; \nu}\left(\pi_{; \nu}^{\diamond} \theta ; t\right)\right]_{n l}=\mathcal{M}_{h, 1 ; \nu}\left(\xi_{n l}(t), \pi_{l ; \nu}^{\diamond} \theta(t)\right), \quad\left[\mathcal{N}_{h, 2 ; \nu}\left(\pi_{; \nu}^{\diamond} \theta ; t\right)\right]_{n l}=\mathcal{M}_{h, 2 ; \nu}\left(\xi_{n l}(t), \pi_{l ; \nu}^{\diamond} \theta(t)\right) .
$$

Notice in particular, that for any $\nu \in\{1, \ldots, 4\}$ we have

$$
\left[\pi_{n l ; \nu}^{\times}-\pi_{n l ; 5}^{\times}\right] \mathrm{J}(h ; t)=\mathrm{J}_{n l}\left(\tau_{\nu} h ; t\right)-\mathrm{J} n l(h ; t)+\mathcal{M}_{h, 1 ; \nu}\left(\xi_{n l}(t), \pi_{l ; \nu}^{\diamond} \theta(t)\right) .
$$

In global form, we write this as

$$
\left[\pi_{; \nu}^{\times}-\pi_{; 5}^{\times}\right] \mathrm{J}(h ; t)=\mathrm{J}\left(\tau_{\nu} h ; t\right)-\mathrm{J}(h ; t)+\mathcal{N}_{h, 1 ; \nu}\left(\pi_{; \nu}^{\diamond} \theta ; t\right) .
$$

Moving on, we use (5.73) to compute

$$
\begin{aligned}
\mathrm{J}_{n l}(L ; t) \pi_{n l}^{\times} \mathrm{J}(h ; t)= & L_{\mu}^{\times} \mathrm{J} n l\left(\tau_{\mu} h ; t\right)+g^{\prime}\left(\Phi\left(\xi_{n l}(t)\right)\right) \mathrm{J} n l(h ; t)+L_{\mu}^{\times} \mathcal{M}_{h, 1 ; \mu}\left(\xi_{n l}(t), \pi_{l ; \mu}^{\diamond} \theta(t)\right) \\
= & L_{\mu}^{\times} \mathrm{J}_{n l}\left(\tau_{\mu} h ; t\right)+g^{\prime}\left(\Phi\left(\xi_{n l}(t)\right)\right) \mathrm{J}_{n l}(h ; t)-L_{\mu}^{\times} \pi_{l ; \mu}^{\diamond} \theta(t) \mathrm{J}_{n l}\left(\tau_{\mu} h^{\prime} ; t\right) \\
& \quad+L_{\mu}^{\times} \mathcal{M}_{h, 2 ; \mu}\left(\xi_{n l}(t) ; \pi_{l ; \mu}^{\diamond} \theta(t)\right) .
\end{aligned}
$$

We remind the reader that according to our summation convention, all three terms featuring $\mu$ in the final identity come with an implicit $\sum_{\mu=1}^{5}$ summation in front. Exploiting the fact that $\pi_{; 5}^{\diamond}=0$ and $L_{\nu}^{\times}=1$ for $1 \leq \nu \leq 4$, we can now write

$$
\mathrm{J}(L, t) \pi^{\times} \mathrm{J}(h ; t)=\mathrm{J}(L \tau h ; t)-\mathrm{J}\left(\pi_{; \mu}^{\diamond} \theta, \tau_{\mu} h^{\prime} ; t\right)+\mathcal{N}_{h, 2 ; \mu}\left(\pi_{; \mu}^{\diamond} \theta ; t\right) .
$$

We now focus on expressions involving $\mathrm{J}(q, h ; t)$. First of all, a short computation shows that

$$
\begin{aligned}
\pi_{n l ; \nu}^{\times} \mathrm{J}(q, h ; t) & =q_{l+\sigma_{\nu}}(t) h\left(\xi_{n+\tau_{\nu}, l+\sigma_{\nu}}(t)\right) \\
& =\pi_{l ; \nu}^{\diamond} q(t) \pi_{n l ; \nu}^{\times} \mathrm{J}(h ; t)+q_{l}(t) \pi_{n l ; \nu}^{\times} \mathrm{J}(h ; t) .
\end{aligned}
$$

Using the expression (5.73) above, we expand this as

$$
\begin{gathered}
\pi_{n l ; \nu}^{\times} \mathrm{J}(q, h ; t)=\pi_{l ; \nu}^{\diamond} q(t)_{\mathrm{J}_{n l}}\left(\tau_{\nu} h ; t\right)+\pi_{l ; \nu}^{\diamond} q(t) \mathcal{M}_{h, 1 ; \nu}\left(\xi_{n l}(t), \pi_{l ; \nu}^{\diamond} \theta(t)\right) \\
+q_{l}(t) \mathrm{J}_{n l}\left(\tau_{\nu} h ; t\right)-q_{l}(t) \pi_{l ; \nu}^{\diamond} \theta(t) \mathrm{J}_{n l}\left(\tau_{\nu} h^{\prime} ; t\right) \\
+q_{l}(t) \mathcal{M}_{h, 2 ; \nu}\left(\xi_{n l}(t), \pi_{l ; \nu}^{\diamond} \theta(t)\right) .
\end{gathered}
$$

In global form, this is

$$
\begin{gathered}
\pi_{; \nu}^{\times} \mathrm{J}(q, h ; t)=\mathrm{J}\left(\pi_{; \nu}^{\diamond} q, \tau_{\nu} h ; t\right)+\pi_{; \nu}^{\diamond} q(t) \mathcal{N}_{h, 1 ; \nu}\left(\pi_{; \nu}^{\diamond} \theta ; t\right) \\
+\mathrm{J}\left(q, \tau_{\nu} h ; t\right)-\mathrm{J}\left(q, \pi_{; \nu}^{\diamond} \theta, \tau_{\nu} h^{\prime} ; t\right) \\
+q(t) \mathcal{N}_{h, 2 ; \nu}\left(\pi_{; \nu}^{\diamond} \theta ; t\right) .
\end{gathered}
$$


At times, it suffices to use the cruder version

$$
\begin{array}{r}
\pi_{; \nu}^{\times} \mathrm{J}(q, h ; t)=\mathrm{J}\left(\pi_{; \nu}^{\diamond} q, \tau_{\nu} h ; t\right)+\pi_{; \nu}^{\diamond} q(t) \mathcal{N}_{h, 1 ; \nu}\left(\pi_{; \nu}^{\diamond} \theta ; t\right) \\
+\mathrm{J}\left(q, \tau_{\nu} h ; t\right)+q(t) \mathcal{N}_{h, 1 ; \nu}\left(\pi_{; \nu}^{\diamond} \theta ; t\right) .
\end{array}
$$

In particular, exploiting the crude identity (5.85), we obtain

$$
\begin{aligned}
{\left[\pi_{; \nu}^{\times}-\pi_{; 5}^{\times}\right] \mathrm{J}(q, h ; t)=} & \mathrm{J}\left(\pi_{; \nu}^{\diamond} q, \tau_{\nu} h ; t\right)+\pi_{; \nu}^{\diamond} q(t) \mathcal{N}_{h, 1 ; \nu}\left(\pi_{; \nu}^{\diamond} \theta ; t\right) \\
& +\mathrm{J}\left(q, \tau_{\nu} h ; t\right)-\mathrm{J}(q, h ; t)+q(t) \mathcal{N}_{h, 1 ; \nu}\left(\pi_{; \nu}^{\diamond} \theta ; t\right) .
\end{aligned}
$$

Moving on, we compute

$$
\begin{aligned}
\mathrm{J}_{n l}(L ; t) \pi_{n l}^{\times} \mathrm{J}(q, h ; t)=L_{\mu}^{\times} & \pi_{l ; \mu}^{\diamond} q(t) \mathrm{J}_{n l}\left(\tau_{\mu} h ; t\right)+L_{\mu}^{\times} \pi_{l ; \mu}^{\diamond} q(t) \mathcal{M}_{h, 1 ; \mu}\left(\xi_{n l}(t), \pi_{l ; \mu}^{\diamond} \theta(t)\right) \\
& +q_{l}(t) \mathrm{J}_{n l}(L ; t) \mathrm{J}_{n l}(\tau h ; t)-q_{l}(t) L_{\mu}^{\times} \pi_{l ; \mu}^{\diamond} \theta(t) \mathrm{J}_{n l}\left(\tau_{\mu} h^{\prime} ; t\right) \\
& +q_{l}(t) L_{\mu}^{\times} \mathcal{M}_{h, 2 ; \mu}\left(\xi_{n l}(t), \pi_{l ; \mu}^{\diamond} \theta(t)\right),
\end{aligned}
$$

which as before can be simplified to

$$
\begin{aligned}
\mathrm{J} n l_{l}(L ; t) \pi_{n l}^{\times} \mathrm{J}(q, h ; t)=\pi_{l ; \mu}^{\diamond} & q(t)_{\mathrm{J} n l}\left(\tau_{\mu} h ; t\right)+\pi_{l ; \mu}^{\diamond} q(t) \mathcal{M}_{h, 1 ; \mu}\left(\xi_{n l}(t), \pi_{l ; \mu}^{\diamond} \theta(t)\right) \\
& +q_{l}(t)_{\mathrm{J} n l}(L ; t) \mathrm{J}_{n l}(\tau h ; t)-q_{l}(t) \pi_{l ; \mu}^{\diamond} \theta(t) \mathrm{J} n l \\
& \left.+\tau_{\mu} h^{\prime} ; t\right) \\
& +q_{l}(t) \mathcal{M}_{h, 2 ; \mu}\left(\xi_{n l}(t), \pi_{l ; \mu}^{\diamond} \theta(t)\right) .
\end{aligned}
$$

In global form, we hence have

$$
\begin{aligned}
& \mathrm{J}(L ; t) \pi^{\times} \mathrm{J}(q, h ; t)=\mathrm{J}\left(\pi_{; \mu}^{\diamond} q, \tau_{\mu} h ; t\right)+\pi_{; \mu}^{\diamond} q(t) \mathcal{N}_{h, 1 ; \mu}\left(\pi_{; \mu}^{\diamond} \theta ; t\right) \\
&+\mathrm{J}(q, L \tau h ; t)-\mathrm{J}\left(q, \pi_{\mu}^{\diamond} \theta, \tau_{\mu} h^{\prime} ; t\right)+q(t) \mathcal{N}_{h, 2 ; \mu}\left(\pi_{; \mu}^{\diamond} \theta ; t\right),
\end{aligned}
$$

which if desired can be simplified to

$$
\begin{array}{r}
\mathrm{J}(L ; t) \pi^{\times} \mathrm{J}(q, h ; t)=\mathrm{J}\left(\pi_{; \mu}^{\diamond} q, \tau_{\mu} h ; t\right)+\pi_{; \mu}^{\diamond} q(t) \mathcal{N}_{h, 1 ; \mu}\left(\pi_{; \mu}^{\diamond} \theta ; t\right) \\
+\mathrm{J}(q, L \tau h ; t)+q(t) \mathcal{N}_{h, 1 ; \mu}\left(\pi_{; \mu}^{\diamond} \theta ; t\right) .
\end{array}
$$

Finally, we discuss the terms involving $\mathrm{J}(p, q, h ; t)$. A short computation shows

$$
\begin{aligned}
\pi_{n l ; \nu}^{\times \mathrm{J}}(p, q, h ; t) & =p_{l+\sigma_{\nu}}(t) q_{l+\sigma_{\nu}}(t) h\left(\xi_{n+\tau_{\nu}, l+\sigma_{\nu}}(t)\right) \\
& =\pi_{l ; \nu}^{\diamond} p(t) \pi_{n l ; \nu}^{\times} \mathrm{J}(q, h ; t)+p_{l}(t) \pi_{n l ; \nu}^{\times} \mathrm{J}(q, h ; t),
\end{aligned}
$$

which using (5.85) expands as

$$
\begin{aligned}
& \pi_{n l ; \nu}^{\times} \mathrm{J}(p, q, h ; t)=\pi_{l ; \nu}^{\diamond} p(t)_{\mathrm{J} n l}\left(\pi_{; \nu}^{\diamond} q, \tau_{\nu} h ; t\right)+\pi_{l ; \nu}^{\diamond} p(t) \pi_{l ; \nu}^{\diamond} q(t) \mathcal{M}_{h, 1 ; \nu}\left(\xi_{n l}(t), \pi_{l ; \nu}^{\diamond} \theta(t)\right) \\
& +\pi_{l ; \nu}^{\diamond} p(t)_{\mathrm{J} n l}\left(q, \tau_{\nu} h ; t\right)+\pi_{l ; \nu}^{\diamond} p(t) q_{l}(t) \mathcal{M}_{h, 1 ; \nu}\left(\xi_{n l}(t), \pi_{l ; \nu}^{\diamond} \theta(t)\right) \\
& +p_{l}(t)_{\mathrm{J}_{n l}}\left(\pi_{; \nu}^{\diamond} q, \tau_{\nu} h ; t\right)+p_{l}(t) \pi_{l ; \nu}^{\diamond} q(t) \mathcal{M}_{h, 1 ; \nu}\left(\xi_{n l}(t), \pi_{l ; \nu}^{\diamond} \theta(t)\right) \\
& +p_{l}(t) \mathbf{J}\left(q, \tau_{\nu} h ; t\right)+p_{l}(t) q_{l}(t) \mathcal{M}_{h, 1 ; \nu}\left(\xi_{n l}(t), \pi_{l ; \nu}^{\diamond} \theta(t)\right) .
\end{aligned}
$$

Inspection of this expression readily yields

$$
\begin{gathered}
{\left[\pi_{; \nu}^{\times}-\pi_{; 5}^{\times}\right] \mathrm{J}(p, q, h ; t)=\quad \mathrm{J}\left(\pi_{; \nu}^{\diamond} p, \pi_{; \nu}^{\diamond} q, \tau_{\nu} h ; t\right)+\pi_{; \nu}^{\diamond} p(t) \pi_{; \nu}^{\diamond} q(t) \mathcal{N}_{h, 1 ; \nu}\left(\pi_{; \nu}^{\diamond} \theta ; t\right)} \\
\left.+\pi_{; \nu}^{\diamond} p(t), q, \tau_{\nu} h ; t\right)+\pi_{; \nu}^{\diamond} p(t) q(t) \mathcal{N}_{h, 1 ; \nu}\left(\pi_{; \nu}^{\diamond} \theta ; t\right) \\
+\mathrm{J}\left(p, \pi_{; \nu}^{\diamond} q, \tau_{\nu} h ; t\right)+p(t) \pi_{; \nu}^{\diamond} q(t) \mathcal{N}_{h, 1 ; \nu}\left(\pi_{; \nu}^{\diamond} \theta ; t\right) \\
\quad+\mathrm{J}\left(p, q, \tau_{\nu} h ; t\right)-\mathrm{J}(p, q, h ; t)+p(t) q(t) \mathcal{N}_{h, 1 ; \nu}\left(\pi_{; \nu}^{\diamond} \theta ; t\right) .
\end{gathered}
$$


In addition, we can compute

$$
\begin{array}{r}
\mathrm{J}_{n l}(L ; t) \pi_{n l}^{\times} \mathrm{J}(p, q, h ; t)=\pi_{l ; \mu}^{\diamond} p(t)_{\mathrm{J} n l}\left(\pi_{; \mu}^{\diamond} q, \tau_{\mu} h ; t\right)+\pi_{l ; \mu}^{\diamond} p(t) \pi_{l ; \mu}^{\diamond} q(t) \mathcal{M}_{h, 1 ; \mu}\left(\xi_{n l}(t), \pi_{l ; \mu}^{\diamond} \theta(t)\right) \\
+\pi_{l ; \mu}^{\diamond} p(t)_{\mathrm{J} n l}\left(q, \tau_{\mu} h ; t\right)+\pi_{l ; \mu}^{\diamond} p(t) q_{l}(t) \mathcal{M}_{h, 1 ; \mu}\left(\xi_{n l}(t), \pi_{l ; \mu}^{\diamond} \theta(t)\right) \\
+p_{l}(t)_{\mathrm{J} n l}\left(\pi_{; ; \mu}^{\diamond} q, \tau_{\mu} h ; t\right)+p_{l}(t) \pi_{l ; \mu}^{\diamond} q(t) \mathcal{M}_{h, 1 ; \mu}\left(\xi_{n l}(t), \pi_{l ; \mu}^{\diamond} \theta(t)\right) \\
\quad+p_{l}(t) q_{l}(t)_{\mathrm{J} n l}(L \tau h ; t)+p_{l}(t) q_{l}(t) \mathcal{M}_{h, 1 ; \mu}\left(\xi_{n l}(t), \pi_{l ; \mu}^{\diamond} \theta(t)\right),
\end{array}
$$

which can be rewritten in global form as

$$
\begin{aligned}
\mathrm{J}(L ; t) \pi^{\times} \mathrm{J}(p, q, h ; t)= & \mathrm{J}\left(\pi_{; \mu}^{\diamond} p(t), \pi_{; \mu}^{\diamond} q, \tau_{\mu} h ; t\right)+\pi_{; \mu}^{\diamond} p(t) \pi_{; \mu}^{\diamond} q(t) \mathcal{N}_{h, 1 ; \mu}\left(\pi_{; \mu}^{\diamond} \theta ; t\right) \\
& +\mathrm{J}\left(\pi_{; \mu}^{\diamond} p(t), q, \tau_{\mu} h ; t\right)+\pi_{; \mu}^{\diamond} p(t) q(t) \mathcal{N}_{h, 1 ; \mu}\left(\pi_{; \mu}^{\diamond} \theta ; t\right) \\
& +\mathrm{J}\left(p, \pi_{; \mu}^{\diamond} q, \tau_{\mu} h ; t\right)+p(t) \pi_{; \mu}^{\diamond} q(t) \mathcal{N}_{h, 1 ; \mu}\left(\pi_{; \mu}^{\diamond} \theta ; t\right) \\
& +\mathrm{J}(p, q, L \tau h ; t)+p(t) q(t) \mathcal{N}_{h, 1 ; \mu}\left(\pi_{; \mu}^{\diamond} \theta ; t\right) .
\end{aligned}
$$

\subsection{The Ansatz}

In this subsection we introduce the basic form of the sub-solution that we will analyze and perform some preliminary computations pertaining to the differential inequality that sub-solutions must satisfy. In particular, throughout this subsection we fix three external functions

$$
\theta \in C^{1}\left([0, \infty), \ell^{\infty}(\mathbb{Z} ; \mathbb{R})\right), \quad z \in C^{1}([0, \infty), \mathbb{R}), \quad Z \in C^{1}([0, \infty), \mathbb{R})
$$

and consider fifty-five auxilliary functions

$$
p_{\nu}^{\diamond} \in B C^{2}(\mathbb{R}, \mathbb{R}), \quad p_{\nu \nu^{\prime}}^{\diamond \diamond} \in B C^{2}(\mathbb{R}, \mathbb{R}), \quad q_{\nu \nu^{\prime}}^{\diamond \diamond} \in B C^{2}(\mathbb{R}, \mathbb{R})
$$

that will be determined later on in this subsection.

Our Ansatz can be written as

$$
\begin{aligned}
u_{n l}^{-}(t)=\Phi(n & \left.+c t-\theta_{l}(t)-Z(t)\right)+\pi_{l ; \mu}^{\diamond} \theta(t) p_{\mu}^{\diamond}\left(n+c t-\theta_{l}(t)-Z(t)\right) \\
& +\pi_{l ; \mu \mu^{\prime}}^{\diamond} \theta(t) p_{\mu \mu^{\prime}}^{\diamond \diamond}\left(n+c t-\theta_{l}(t)-Z(t)\right) \\
& +\pi_{l ; \mu}^{\diamond} \theta(t) \pi_{l ; \mu^{\prime}}^{\diamond} \theta(t) q_{\mu \mu^{\prime}}^{\diamond}\left(n+c t-\theta_{l}(t)-Z(t)\right)-z(t) .
\end{aligned}
$$

Upon writing

$$
\xi_{n l}(t)=n+c t-\theta_{l}(t)-Z(t),
$$

our Ansatz can be rephrased in the global form

$$
u^{-}(t)=\mathrm{J}(\Phi ; t)+\mathrm{J}\left(\pi_{; \mu}^{\diamond} \theta, p_{\mu}^{\diamond} ; t\right)+\mathrm{J}\left(\pi_{; \mu \mu^{\prime}}^{\diamond} \theta, p_{\mu \mu^{\prime}}^{\diamond} ; t\right)+\mathrm{J}\left(\pi_{; \mu}^{\diamond} \theta, \pi_{; \mu^{\prime}}^{\diamond} \theta, q_{\mu \mu^{\prime}}^{\diamond} ; t\right)-z(t) .
$$

We note that $\mathrm{J}(\Phi ; t)-z(t)$ can be seen as the direct lifting of the PDE sub-solution used in [9] to the discrete setting. The terms $\mathrm{J}\left(\pi_{; \mu}^{\diamond} \theta, p_{\mu}^{\diamond} ; t\right)$ correspond to those that were explicitly discussed in $\S 1$, which allowed a factor $\Phi^{\prime}(\xi)$ to be pulled off from all first differences in $\theta$ appearing in the residual. The remaining terms in (5.100) are designed to allow a similar factorization for all second order differences and certain problematic products of first order differences.

As a first preparation, we introduce the nonlinear expression

$$
\begin{aligned}
\mathcal{R}_{\mathcal{N} ; \nu}\left(\pi^{\diamond} \theta, \pi^{\diamond} \theta ; t\right)= & {\left[\pi_{; \nu}^{\times}-\pi_{; 5}^{\times}\right] u^{-}(t) } \\
= & \mathcal{R}_{\mathcal{N} ; \Phi ; \nu}\left(\pi^{\diamond} \theta ; t\right)+\mathcal{R}_{\mathcal{N} ; p^{\diamond} ; \nu}\left(\pi^{\diamond} \theta, \pi^{\diamond \diamond} \theta ; t\right) \\
& \quad+\mathcal{R}_{\mathcal{N} ; p^{\diamond \diamond ; \nu}}\left(\pi^{\diamond} \theta, \pi^{\diamond \diamond} \theta ; t\right)+\mathcal{R}_{\mathcal{N} ; q^{\diamond} ; \nu}\left(\pi^{\diamond} \theta, \pi^{\diamond \diamond} \theta ; t\right),
\end{aligned}
$$


in which we have defined

$$
\begin{aligned}
& \mathcal{R}_{\mathcal{N} ; \Phi ; \nu}\left(\pi^{\diamond} \theta ; t\right)=\left[\pi_{; \nu}^{\times}-\pi_{; 5}^{\times}\right] \mathrm{J}(\Phi ; t), \\
& \mathcal{R}_{\mathcal{N} ; p^{\diamond} ; \nu}\left(\pi^{\diamond} \theta, \pi^{\diamond \diamond} \theta ; t\right)=\left[\pi_{; \nu}^{\times}-\pi_{; 5}^{\times}\right] \mathrm{J}\left(\pi_{; \mu^{\prime}}^{\diamond} \theta, p_{\mu^{\prime}}^{\diamond} ; t\right), \\
& \mathcal{R}_{\mathcal{N} ; p^{\diamond} ; \nu}\left(\pi^{\diamond} \theta, \pi^{\diamond \diamond} \theta ; t\right)=\left[\pi_{; \nu}^{\times}-\pi_{; 5}^{\times}\right]_{\mathrm{J}}\left(\pi_{; \mu^{\prime} \mu^{\prime \prime}}^{\diamond} \theta, p_{\mu^{\prime} \mu^{\prime \prime}}^{\diamond} ; t\right), \\
& \mathcal{R}_{\mathcal{N} ; q^{\diamond} ; \nu}\left(\pi^{\diamond} \theta, \pi^{\diamond \diamond} \theta ; t\right)=\left[\pi_{; \nu}^{\times}-\pi_{; 5}^{\times}\right] \mathrm{J}\left(\pi_{; \mu^{\prime}}^{\diamond} \theta, \pi_{; \mu^{\prime \prime}}^{\diamond} \theta, q_{\mu^{\prime} \mu^{\prime \prime}}^{\diamond} ; t\right) .
\end{aligned}
$$

Using the expressions obtained in $\S 5.2$, we now compute

$$
\begin{aligned}
& \mathcal{R}_{\mathcal{N} ; \Phi ; \nu}\left(\pi^{\diamond} \theta ; t\right)=\mathrm{J}\left(\tau_{\nu} \Phi ; t\right)-\mathrm{J}(\Phi ; t)+\mathcal{N}_{\Phi, 1 ; \nu}\left(\pi_{; \nu}^{\diamond} \theta(t) ; t\right) \\
& \mathcal{R}_{\mathcal{N} ; p^{\diamond} ; \nu}\left(\pi^{\diamond} \theta, \pi^{\diamond \diamond} \theta ; t\right)=\mathrm{J}\left(\pi_{; \mu^{\prime} \nu}^{\diamond \diamond} \theta, p_{\mu^{\prime}}^{\diamond} ; t\right)+\pi_{\mu^{\prime} \nu}^{\diamond} \theta(t) \mathcal{N}_{p_{\mu^{\prime}}, 1 ; \nu}\left(\pi_{; \nu}^{\diamond} \theta(t) ; t\right) \\
& +\pi^{\diamond}\left(\pi_{\mu^{\prime}} \theta, \tau_{\nu} p_{\mu^{\prime}}^{\diamond} ; t\right)-\mathrm{J}\left(\pi_{\mu^{\prime}}^{\diamond} \theta, p_{\mu^{\prime}}^{\diamond} ; t\right) \\
& +\pi_{\mu^{\prime}}^{\diamond} \theta(t) \mathcal{N}_{p_{\mu^{\prime}}^{\diamond}, 1 ; \nu}\left(\pi_{; \nu}^{\diamond} \theta(t)\right),
\end{aligned}
$$

together with

$$
\begin{aligned}
& \mathcal{R}_{\mathcal{N} ; p^{\diamond} ; \nu}\left(\pi^{\diamond} \theta, \pi^{\diamond \diamond} \theta ; t\right)=\mathrm{J}\left(\pi_{; \mu^{\prime} \mu^{\prime \prime} \nu}^{\diamond \diamond \diamond} \theta, p_{\mu^{\prime} \mu^{\prime \prime}}^{\diamond \diamond} ; t\right)+\pi_{\mu^{\prime} \mu^{\prime \prime} \nu}^{\diamond \diamond} \theta(t) \mathcal{N}_{p_{\mu^{\prime} \mu^{\prime \prime}}^{\diamond \diamond}, 1 ; \nu}\left(\pi_{; \nu}^{\diamond} \theta(t) ; t\right) \\
& +\mathrm{J}\left(\pi_{\mu^{\prime} \mu^{\prime \prime}}^{\diamond} \theta, \tau_{\nu} p_{\mu^{\prime} \mu^{\prime \prime}}^{\diamond} ; t\right)-\mathrm{J}\left(\pi_{\mu^{\prime} \mu^{\prime \prime}}^{\diamond \diamond} \theta, p_{\mu^{\prime} \mu^{\prime \prime}}^{\diamond} ; t\right) \\
& +\pi_{\mu^{\prime} \mu^{\prime \prime}}^{\diamond} \theta(t) \mathcal{N}_{p_{\mu^{\prime} \mu^{\prime \prime}}^{\diamond ;}, i \nu}\left(\pi_{; \nu}^{\diamond} \theta(t)\right) \text {, } \\
& \mathcal{R}_{\mathcal{N} ; q^{\diamond \diamond} ; \nu}\left(\pi^{\diamond} \theta, \pi^{\diamond \diamond} \theta ; t\right)=\mathrm{J}\left(\pi_{\mu^{\prime} \nu}^{\diamond \diamond} \theta, \pi_{\mu^{\prime \prime} \nu}^{\diamond \diamond} \theta, \tau_{\nu} q_{\mu^{\prime} \mu^{\prime \prime}}^{\diamond \diamond} ; t\right)+\pi_{\mu^{\prime} \nu}^{\diamond} \theta(t) \pi_{\mu^{\prime \prime} \nu}^{\diamond \diamond} \theta(t) \mathcal{N}_{q_{\mu^{\prime} \mu^{\prime \prime}}^{\diamond \diamond}, 1 ; \nu}\left(\pi_{; \nu}^{\diamond} \theta ; t\right) \\
& +\mathrm{J}\left(\pi_{\mu^{\prime} \nu}^{\diamond \diamond} \theta, \pi_{\mu^{\prime \prime}}^{\diamond} \theta, \tau_{\nu} q_{\mu^{\prime} \mu^{\prime \prime}}^{\diamond} ; t\right)+\pi_{\mu^{\prime} \nu}^{\diamond} \theta(t) \pi_{\mu^{\prime \prime}}^{\diamond} \theta(t) \mathcal{N}_{q_{\mu^{\prime} \mu^{\prime \prime}}^{\diamond}, 1 ; \nu}\left(\pi_{; \nu}^{\diamond} \theta ; t\right) \\
& +\mathrm{J}\left(\pi_{\mu^{\prime}}^{\diamond} \theta, \pi_{\mu^{\prime \prime} \nu}^{\diamond \diamond} \theta, \tau_{\nu} q_{\mu^{\prime} \mu^{\prime \prime}}^{\diamond} ; t\right)+\pi_{\mu^{\prime}}^{\diamond} \theta(t) \pi_{\mu^{\prime \prime} \nu}^{\diamond \diamond} \theta(t) \mathcal{N}_{q_{\mu^{\prime} \mu^{\prime \prime}}^{\diamond}, 1 ; \nu}\left(\pi_{; \nu}^{\diamond} \theta ; t\right) \\
& +\mathrm{J}\left(\pi_{\mu^{\prime}}^{\diamond} \theta, \pi_{\mu^{\prime \prime}}^{\diamond} \theta, \tau_{\nu} q_{\mu^{\prime} \mu^{\prime \prime}}^{\diamond} ; t\right)-\mathrm{J}\left(\pi_{\mu^{\prime}}^{\diamond} \theta, \pi_{\mu^{\prime \prime}}^{\diamond} \theta, q_{\mu^{\prime} \mu^{\prime \prime}}^{\diamond \diamond} ; t\right) \\
& +\pi_{\mu^{\prime}}^{\diamond} \theta(t) \pi_{\mu^{\prime \prime}}^{\diamond} \theta(t) \mathcal{N}_{q_{\mu^{\prime} \mu^{\prime \prime}}^{\diamond \diamond}, 1 ; \nu}\left(\pi_{; \nu}^{\diamond} \theta ; t\right) \text {. }
\end{aligned}
$$

We now turn to the main task in this subsection, which is to consider the quantity

$$
\mathcal{J}_{n l}^{-}(t)=\dot{u}_{n l}^{-}(t)-\left[\Delta^{\times} u^{-}(t)\right]_{n l}-g\left(u_{n l}^{-}(t)\right)
$$

and determine suitable choices for the functions (5.97). To this end, we decompose $\mathcal{J}_{n l}^{-}(t)$ as

$$
\begin{aligned}
\mathcal{J}_{n l}^{-}(t)= & \dot{u}_{n l}^{-}(t)-L^{\times} \pi_{n l}^{\times} u^{-}(t)-g\left(u_{n l}^{-}(t)\right) \\
= & \dot{u}_{n l}^{-}(t)-\mathrm{J} n l(L ; t) \pi_{n l}^{\times} u^{-}(t)+g^{\prime}\left(\Phi\left(\xi_{n l}(t)\right)\right) u_{n l}^{-}(t)-g\left(u_{n l}^{-}(t)\right) \\
= & \left.\dot{u}_{n l}^{-}(t)-\mathrm{J} n l(L ; t)\right) \pi_{n l}^{\times} u^{-}(t)+g^{\prime}\left(\Phi\left(\xi_{n l}(t)\right)\right)\left[u_{n l}^{-}(t)-\Phi\left(\xi_{n l}(t)\right)\right]-g\left(u_{n l}^{-}(t)\right) \\
& \quad+g^{\prime}\left(\Phi\left(\xi_{n l}(t)\right)\right) \Phi\left(\xi_{n l}(t)\right) .
\end{aligned}
$$

For convenience, we rephrase this as

$$
\begin{gathered}
\mathcal{J}^{-}(t)=\dot{u}^{-}(t)-\mathrm{J}(L ; t) \pi^{\times} u^{-}(t)+\mathrm{J}\left(g^{\prime}(\Phi) ; t\right)\left[u^{-}(t)-\mathrm{J}(\Phi ; t)\right]-g\left(u^{-}(t)\right) \\
+\mathrm{J}(L \tau \Phi ; t)-L^{\times} \mathrm{J}(\tau \Phi ; t)
\end{gathered}
$$

and carefully study each of the terms. 
First of all, a short computation shows that

$$
\begin{aligned}
\dot{u}^{-}(t)=c \mathrm{~J}\left(\Phi^{\prime} ; t\right) & -\mathrm{J}\left(\dot{\theta}+\dot{Z}, \Phi^{\prime} ; t\right)-\dot{z}(t) \\
& +\mathrm{J}\left(\pi_{; \mu}^{\diamond} \dot{\theta}, p_{\mu}^{\diamond} ; t\right)+c \mathrm{~J}\left(\pi_{; \mu}^{\diamond} \theta, D p_{\mu}^{\diamond} ; t\right)-\mathrm{J}\left(\dot{\theta}+\dot{Z}, \pi_{; \mu}^{\diamond} \theta, D p_{\mu}^{\diamond} ; t\right) \\
& +\mathrm{J}\left(\pi_{; \mu \mu^{\prime}}^{\diamond \diamond} \dot{\theta}, p_{\mu \mu^{\prime}}^{\diamond \diamond} ; t\right)+c \mathrm{~J}\left(\pi_{; \mu \mu^{\prime}}^{\diamond \diamond} \theta, D p_{\mu \mu^{\prime}}^{\diamond \diamond} ; t\right)-\mathrm{J}\left(\dot{\theta}+\dot{Z}, \pi_{; \mu \mu^{\prime}}^{\diamond \diamond} \theta, D p_{\mu \mu^{\prime}}^{\diamond \diamond} ; t\right) \\
& +\mathrm{J}\left(\pi_{; \mu}^{\diamond} \dot{\theta}, \pi_{; \mu^{\prime}}^{\diamond}, q_{\mu \mu^{\prime}}^{\diamond \diamond} ; t\right)+\mathrm{J}\left(\pi_{; \mu}^{\diamond} \theta, \pi_{; \mu^{\prime}}^{\diamond} \dot{\theta}, q_{\mu \mu^{\prime}}^{\diamond \diamond} ; t\right) \\
& \quad+c \mathrm{~J}\left(\pi_{; \mu}^{\diamond} \theta, \pi_{; \mu^{\prime}}^{\diamond} \theta, D q_{\mu \mu^{\prime}}^{\diamond \diamond} ; t\right)-\mathrm{J}\left(\dot{\theta}+\dot{Z}, \pi_{; \mu}^{\diamond} \theta, \pi_{; \mu^{\prime}}^{\diamond} \theta, D q_{\mu \mu^{\prime}}^{\diamond \diamond} ; t\right) .
\end{aligned}
$$

For later use, we introduce the fifty-five functions

$$
f_{p ; \nu}^{\diamond} \in B C^{1}(\mathbb{R}, \mathbb{R}), \quad f_{p ; \nu \nu^{\prime}}^{\diamond} \in B C^{1}(\mathbb{R}, \mathbb{R}), \quad f_{q ; \nu \nu^{\prime}}^{\diamond \diamond} \in B C^{1}(\mathbb{R}, \mathbb{R})
$$

that are defined by

$$
f_{p ; \nu}^{\diamond}=\mathcal{L}_{0} p^{\diamond}, \quad f_{p ; \nu \nu^{\prime}}^{\diamond \diamond}=\mathcal{L}_{0} p_{\nu \nu^{\prime}}^{\diamond}, \quad f_{q ; \nu \nu^{\prime}}^{\diamond \diamond}=\mathcal{L}_{0} q_{\nu \nu^{\prime}}^{\diamond},
$$

in which we have recalled the linear operator

$$
\begin{gathered}
{\left[\mathcal{L}_{0} v\right](\xi)=-c v^{\prime}(\xi)+v\left(\xi+\sigma_{h}\right)+v\left(\xi+\sigma_{v}\right)+v\left(\xi-\sigma_{h}\right)+v\left(\xi-\sigma_{v}\right)-4 v(\xi)} \\
+g^{\prime}(\Phi(\xi)) v(\xi) .
\end{gathered}
$$

Notice in particular that if $\mathcal{L}_{0} v=f$, then

$$
c_{J_{n l}}\left(v^{\prime} ; t\right)=\mathrm{J}_{n l}(L ; t) \mathrm{J}_{n l}(\tau v ; t)-f\left(\xi_{n l}(t)\right),
$$

which allows us to write

$$
c_{\mathrm{J}}\left(v^{\prime} ; t\right)=\mathrm{J}(L \tau v ; t)-\mathrm{J}(f ; t) .
$$

In addition, the wave profile equation implies that for any $t \geq 0$ we have

$$
c \Phi^{\prime}\left(\xi_{n l}(t)\right)=L_{\mu}^{\times}\left[\tau_{\mu} \Phi\right]\left(\xi_{n l}(t)\right)+g\left(\Phi\left(\xi_{n l}(t)\right)\right),
$$

which implies that

$$
\begin{aligned}
c \mathrm{~J}\left(\Phi^{\prime} ; t\right) & =L_{\mu}^{\times} \mathrm{J}\left(\tau_{\mu} \Phi ; t\right)+\mathrm{J}(g(\Phi) ; t) \\
& =L^{\times} \mathrm{J}(\tau \Phi ; t)+\mathrm{J}(g(\Phi) ; t) .
\end{aligned}
$$

With this hand, we can expand $\dot{u}^{-}(t)$ as

$$
\begin{aligned}
& \dot{u}^{-}(t)=L^{\times}(\tau\Phi ; t)+\mathrm{J}(g(\Phi) ; t)-\mathrm{J}\left(\dot{\theta}+\dot{Z}, \Phi^{\prime} ; t\right)-\dot{z}(t) \\
&+\mathrm{J}(\left.\pi_{; \mu}^{\diamond} \dot{\theta}, p_{\mu}^{\diamond} ; t\right)-\mathrm{J}\left(\dot{\theta}+\dot{Z}, \pi_{; \mu}^{\diamond} \theta, D p_{\mu}^{\diamond} ; t\right) \\
&+\mathrm{J}\left(\pi_{; \mu}^{\diamond} \theta, L \tau p_{\mu}^{\diamond} ; t\right)-\mathrm{J}\left(\pi_{; \mu}^{\diamond} \theta, f_{p ; \mu}^{\diamond} ; t\right) \\
&+\mathrm{J}(\left.\pi_{; \mu \mu^{\prime}}^{\diamond} \dot{\theta}, p_{\mu \mu^{\prime}}^{\diamond \diamond} ; t\right)-\mathrm{J}\left(\dot{\theta}+\dot{Z}, \pi_{; \mu \mu^{\prime}}^{\diamond} \theta, D p_{\mu \mu^{\prime}}^{\diamond \diamond} ; t\right) \\
&+\mathrm{J}\left(\pi_{; \mu \mu^{\prime}}^{\diamond \diamond} \theta, L \tau p_{\mu \mu^{\prime}}^{\diamond \diamond} ; t\right)-\mathrm{J}\left(\pi_{; \mu \mu^{\prime}}^{\diamond \diamond} \theta, f_{p ; \mu \mu^{\prime}}^{\diamond \diamond} ; t\right) \\
&+\mathrm{J}\left(\pi_{; \mu}^{\diamond} \dot{\theta}, \pi_{; \mu^{\prime}}^{\diamond} \theta, q_{\mu \mu^{\prime}}^{\diamond \diamond} ; t\right)+\mathrm{J}\left(\pi_{; \mu}^{\diamond} \theta, \pi_{; \mu^{\prime}}^{\diamond}, q_{\mu \mu^{\prime}}^{\diamond \diamond} ; t\right) \\
& \quad-\mathrm{J}\left(\dot{\theta}+\dot{Z}, \pi_{; \mu}^{\diamond} \theta, \pi_{; \mu^{\prime}}^{\diamond} \theta, D q_{\mu \mu^{\prime}}^{\diamond \diamond} ; t\right) \\
&+\mathrm{J}\left(\pi_{; \mu}^{\diamond} \theta, \pi_{; \mu^{\prime}}^{\diamond} \theta, L \tau q_{\mu \mu^{\prime}}^{\diamond \diamond} ; t\right)-\mathrm{J}\left(\pi_{; \mu}^{\diamond} \theta, \pi_{; \mu^{\prime}}^{\diamond} \theta, f_{q ; \mu \mu^{\prime}}^{\diamond \diamond} ; t\right) .
\end{aligned}
$$


Moving on to the second term in (5.107), we use the computations in $\S 5.2$ to compute

$$
\begin{aligned}
& \mathrm{J}(L ; t) \pi^{\times} u^{-}(t)=\mathrm{J}(L, \tau \Phi ; t)-\mathrm{J}\left(\pi_{\mu}^{\diamond} \theta, \tau_{\mu} \Phi^{\prime} ; t\right)+\mathcal{N}_{\Phi, 2 ; \mu}\left(\pi_{; \mu}^{\diamond} \theta ; t\right)-\mathrm{J}(L ; t) z(t) \\
& +\mathrm{J}\left(\pi_{; \mu \mu^{\prime}}^{\diamond} \theta, \tau_{\mu^{\prime}} p_{\mu}^{\diamond} ; t\right)+\pi_{; \mu \mu^{\prime}}^{\diamond \diamond} \theta(t) \mathcal{N}_{p_{\mu}, 1 ; \mu^{\prime}}\left(\pi_{; \mu^{\prime}}^{\diamond} \theta ; t\right) \\
& \mathrm{H}_{\mathrm{J}}\left(\pi_{; \mu}^{\diamond} \theta, L \tau p_{\mu}^{\diamond} ; t\right)-\mathrm{J}\left(\pi_{; \mu}^{\diamond} \theta, \pi_{; \mu^{\prime}}^{\diamond} \theta, \tau_{\mu^{\prime}} D p_{\mu}^{\diamond} ; t\right) \\
& +\pi_{; \mu}^{\diamond} \theta(t) \mathcal{N}_{p_{\mu}^{\diamond}, 2 ; \mu^{\prime}}\left(\pi_{; \mu^{\prime}}^{\diamond} \theta ; t\right) \\
& +\mathrm{J}\left(\pi_{; \mu \mu^{\prime} \mu^{\prime \prime}}^{\diamond \diamond \diamond} \theta, \tau_{\mu^{\prime \prime}} p_{\mu \mu^{\prime}}^{\diamond} ; t\right)+\pi_{; \mu \mu^{\prime} \mu^{\prime \prime}}^{\diamond \diamond \diamond} \theta(t) \mathcal{N}_{p_{\mu \mu^{\prime}}^{\diamond}, 1 ; \mu^{\prime \prime}}\left(\pi_{; \mu^{\prime \prime}}^{\diamond} \theta ; t\right) \\
& +\mathrm{J}\left(\pi_{; \mu \mu^{\prime}}^{\diamond} \theta, L \tau p_{\mu \mu^{\prime}}^{\diamond} ; t\right)+\pi_{; \mu \mu^{\prime}}^{\diamond} \theta(t) \mathcal{N}_{p_{\mu \mu^{\prime}}^{\diamond}, 1 ; \mu^{\prime \prime}}\left(\pi_{; \mu^{\prime \prime}}^{\diamond} \theta ; t\right) \\
& +\mathrm{J}\left(\pi_{\mu \mu^{\prime \prime}}^{\diamond} \theta, \pi_{\mu^{\prime} \mu^{\prime \prime}}^{\diamond \diamond} \theta, \tau_{\mu^{\prime \prime}} q_{\mu \mu^{\prime}}^{\diamond} ; t\right)+\pi_{\mu \mu^{\prime \prime}}^{\diamond \diamond} \theta(t) \pi_{\mu^{\prime} \mu^{\prime \prime}}^{\diamond \diamond} \theta(t) \mathcal{N}_{q_{\mu \mu^{\prime}} \diamond 1 ; \mu^{\prime \prime}}\left(\pi_{; \mu^{\prime \prime}}^{\diamond} \theta ; t\right) \\
& +\mathrm{J}\left(\pi_{\mu \mu^{\prime \prime}}^{\diamond \diamond} \theta, \pi_{\mu^{\prime}}^{\diamond} \theta, \tau_{\mu^{\prime \prime}} q_{\mu \mu^{\prime}}^{\diamond \diamond} ; t\right)+\pi_{\mu \mu^{\prime \prime}}^{\diamond \diamond} \theta(t) \pi_{\mu^{\prime}}^{\diamond} \theta(t) \mathcal{N}_{q_{\mu \mu^{\prime}}^{\diamond}, 1 ; \mu^{\prime \prime}}\left(\pi_{; \mu^{\prime \prime}}^{\diamond} \theta ; t\right) \\
& +\mathrm{J}\left(\pi_{\mu}^{\diamond} \theta, \pi_{\mu^{\prime} \mu^{\prime \prime}}^{\diamond} \theta, \tau_{\mu^{\prime \prime}} q_{\mu \mu^{\prime}}^{\diamond} ; t\right)+\pi_{\mu}^{\diamond} \theta(t) \pi_{\mu^{\prime} \mu^{\prime \prime}}^{\diamond} \theta(t) \mathcal{N}_{q_{\mu \mu^{\prime}}, 1 ; \mu^{\prime \prime}}\left(\pi_{; \mu^{\prime \prime}}^{\diamond} \theta ; t\right) \\
& +\mathrm{J}\left(\pi_{\mu}^{\diamond} \theta, \pi_{\mu^{\prime}}^{\diamond} \theta, L \tau q_{\mu \mu^{\prime}}^{\diamond}, t\right)+\pi_{\mu}^{\diamond} \theta(t) \pi_{\mu^{\prime}}^{\diamond} \theta(t) \mathcal{N}_{q_{\mu \mu^{\prime}}^{\diamond \diamond}, 1 ; \mu^{\prime \prime}}\left(\pi_{; \mu^{\prime \prime}}^{\diamond} \theta ; t\right) \text {. }
\end{aligned}
$$

Comparing (5.107), (5.116) and (5.117), we see that a fair number of terms cancel. In order to organize the remaining terms, we first introduce for $v \in \ell^{\infty}\left(\mathbb{Z}^{2} ; \mathbb{R}\right)$ the nonlinear expression

$$
\mathcal{G}_{0}(v ; t)=-g(\mathrm{~J}(\Phi ; t)+v)+g(\mathrm{~J}(\Phi ; t))+g^{\prime}(\mathrm{J}(\Phi ; t)) v \quad \in \ell^{\infty}\left(\mathbb{Z}^{2} ; \mathbb{R}\right),
$$

which measures the purely nonlinear part of $g$ near the wave $\Phi\left(\xi_{n l}(t)\right)$. Utilizing $\mathcal{G}_{0}$, we now define the nonlinear expression

$$
\begin{gathered}
\mathcal{R}_{1}\left(\pi^{\diamond} \theta, \pi^{\diamond} \theta, z ; t\right)=\mathcal{G}_{0}\left(\mathrm{~J}\left(\pi_{; \mu}^{\diamond} \theta, p_{\mu}^{\diamond} ; t\right)+\mathrm{J}\left(\pi_{\mu \mu^{\prime}}^{\diamond \diamond} \theta, p_{\mu \mu^{\prime}}^{\diamond} ; t\right)+\mathrm{J}\left(\pi_{\mu}^{\diamond} \theta, \pi_{\mu^{\prime}}^{\diamond} \theta, q_{\mu \mu^{\prime}}^{\diamond \diamond} ; t\right)-z(t) ; t\right) \\
-\mathcal{N}_{\Phi, 2 ; \mu}\left(\pi_{; \mu}^{\diamond} \theta ; t\right) .
\end{gathered}
$$

In addition, we define the nonlinear expressions

$$
\begin{aligned}
& \mathcal{R}_{2}\left(\dot{Z}, \pi^{\diamond} \theta, \pi^{\diamond} \theta ; t\right)=-\mathrm{J}\left(\dot{Z}, \pi_{; \mu}^{\diamond} \theta, D p_{\mu}^{\diamond} ; t\right)-\mathrm{J}\left(\dot{Z}, \pi_{; \mu \mu^{\prime}}^{\diamond \diamond} \theta, D p_{\mu \mu^{\prime}}^{\diamond \diamond} ; t\right), \\
& \mathcal{R}_{3}\left(\dot{\theta}, \pi^{\diamond \diamond} \theta ; t\right) \\
& \mathcal{R}_{4}\left(\pi^{\diamond} \dot{\theta}, \pi^{\diamond} \theta ; t\right)=-\mathrm{J}\left(\dot{\theta}, \pi_{; \mu \mu^{\prime}}^{\diamond \diamond} \theta, D p_{\mu \mu^{\prime}}^{\diamond \diamond} ; t\right), \\
& =\mathrm{J}\left(\pi_{; \mu}^{\diamond} \dot{\theta}, \pi_{; \mu^{\prime}}^{\diamond} \theta, q_{\mu \mu^{\prime}}^{\diamond} ; t\right)+\mathrm{J}\left(\pi_{; \mu}^{\diamond} \theta, \pi_{; \mu^{\prime}}^{\diamond} \dot{\theta}, q_{\mu \mu^{\prime}}^{\diamond \diamond} ; t\right),
\end{aligned}
$$

together with

$$
\begin{aligned}
& \mathcal{R}_{5}\left(\pi^{\diamond} \theta, \pi^{\diamond \diamond} \theta, \pi^{\diamond \diamond \diamond} \theta ; t\right)=-\pi_{; \mu \mu^{\prime}}^{\diamond \diamond} \theta(t) \mathcal{N}_{p_{\mu}^{\diamond}, 1 ; \mu^{\prime}}\left(\pi_{; \mu^{\prime}}^{\diamond} \theta ; t\right)-\pi_{; \mu}^{\diamond} \theta(t) \mathcal{N}_{p_{\mu}^{\diamond}, 2 ; \mu^{\prime}}\left(\pi_{; \mu^{\prime}}^{\diamond} \theta ; t\right) \\
& -\pi_{; \mu \mu^{\prime} \mu^{\prime \prime}}^{\diamond \diamond} \theta(t) \mathcal{N}_{p_{\mu \mu^{\prime}}^{\diamond}, 1 ; \mu^{\prime \prime}}\left(\pi_{; \mu^{\prime \prime}}^{\diamond} \theta ; t\right)-\pi_{; \mu \mu^{\prime}}^{\diamond} \theta(t) \mathcal{N}_{p_{\mu \mu^{\prime}}, 1 ; \mu^{\prime \prime}}\left(\pi_{; \mu^{\prime \prime}}^{\diamond} \theta ; t\right) \\
& -\mathrm{J}\left(\pi_{\mu \mu^{\prime \prime}}^{\diamond} \theta, \pi_{\mu^{\prime} \mu^{\prime \prime}}^{\diamond} \theta, \tau_{\mu^{\prime \prime}} q_{\mu \mu^{\prime}}^{\diamond} ; t\right)-\pi_{\mu \mu^{\prime \prime}}^{\diamond} \theta(t) \pi_{\mu^{\prime} \mu^{\prime \prime}}^{\diamond} \theta(t) \mathcal{N}_{q_{\mu \mu^{\prime}}, 1 ; \mu^{\prime \prime}}\left(\pi_{; \mu^{\prime \prime}}^{\diamond} \theta ; t\right) \\
& -\mathrm{J}\left(\pi_{\mu \mu^{\prime \prime}}^{\diamond \diamond} \theta, \pi_{\mu^{\prime}}^{\diamond} \theta, \tau_{\mu^{\prime \prime}} q_{\mu \mu^{\prime}}^{\diamond \diamond} ; t\right)-\pi_{\mu \mu^{\prime \prime}}^{\diamond \diamond} \theta(t) \pi_{\mu^{\prime}}^{\diamond} \theta(t) \mathcal{N}_{q_{\mu \mu^{\prime}}, 1 ; \mu^{\prime \prime}}\left(\pi_{; \mu^{\prime \prime}}^{\diamond} \theta ; t\right) \\
& -\mathrm{J}\left(\pi_{\mu}^{\diamond} \theta, \pi_{\mu^{\prime} \mu^{\prime \prime}}^{\diamond \diamond} \theta, \tau_{\mu^{\prime \prime}} q_{\mu \mu^{\prime}}^{\diamond \diamond} ; t\right)-\pi_{\mu}^{\diamond} \theta(t) \pi_{\mu^{\prime} \mu^{\prime \prime}}^{\diamond} \theta(t) \mathcal{N}_{q_{\mu \mu^{\prime}}^{\diamond}, 1 ; \mu^{\prime \prime}}\left(\pi_{; \mu^{\prime \prime}}^{\diamond} \theta ; t\right) \\
& -\pi_{\mu}^{\diamond} \theta(t) \pi_{\mu^{\prime}}^{\diamond} \theta(t) \mathcal{N}_{q_{\mu \mu^{\prime}}^{\diamond \diamond}, 1 ; \mu^{\prime \prime}}\left(\pi_{; \mu^{\prime \prime}}^{\diamond} \theta ; t\right) \text {. }
\end{aligned}
$$

Finally, we define the two linear expressions

$$
\begin{aligned}
& \mathcal{E}_{1}\left(\pi^{\diamond \diamond} \dot{\theta} ; t\right)=\mathrm{J}\left(\pi_{; \mu \mu^{\prime}}^{\diamond} \dot{\theta} p_{\mu \mu^{\prime}}^{\diamond \diamond} ; t\right), \\
& \mathcal{E}_{2}\left(\pi^{\diamond \diamond \diamond} \theta ; t\right)=-\mathrm{J}\left(\pi_{; \mu \mu^{\prime} \mu^{\prime \prime}}^{\diamond \diamond \diamond}, \tau_{\mu^{\prime \prime}} p_{\mu \mu^{\prime}}^{\diamond \diamond} ; t\right) .
\end{aligned}
$$


Together, these expressions allow us to write

$$
\begin{aligned}
& \mathcal{J}^{-}(t)=-\mathrm{J}\left(\dot{Z}, \Phi^{\prime} ; t\right)-\dot{z}(t)+\mathrm{J}(L ; t) z(t)-\mathrm{J}\left(\dot{\theta}, \Phi^{\prime} ; t\right)+\mathrm{J}\left(\pi_{\mu}^{\diamond} \theta, \tau_{\mu} \Phi^{\prime} ; t\right)-\mathrm{J}\left(\pi_{; \mu}^{\diamond} \theta, f_{p ; \mu}^{\diamond} ; t\right) \\
&+\mathrm{J}\left(\pi_{; \mu}^{\diamond} \dot{\theta}, p_{\mu}^{\diamond} ; t\right)-\mathrm{J}\left(\pi_{; \mu \mu^{\prime}}^{\diamond} \theta, \tau_{\mu^{\prime}} p_{\mu}^{\diamond} ; t\right)-\mathrm{J}\left(\pi_{; \mu \mu^{\prime}}^{\diamond \diamond} \theta, f_{p ; \mu \mu^{\prime}}^{\diamond \diamond t)}\right. \\
&-\mathrm{J}\left(\dot{\theta}, \pi_{; \mu}^{\diamond} \theta, D p_{\mu}^{\diamond} ; t\right)+\mathrm{J}\left(\pi_{; \mu}^{\diamond} \theta, \pi_{; \mu^{\prime}}^{\diamond} \theta, \tau_{\mu^{\prime}} D p_{\mu}^{\diamond} ; t\right)-\mathrm{J}\left(\pi_{; \mu}^{\diamond} \theta, \pi_{; \mu^{\prime}}^{\diamond} \theta, f_{q ; \mu \mu^{\prime}}^{\diamond \diamond}, t\right) \\
&+\mathcal{R}_{1}\left(\pi^{\diamond} \theta, \pi^{\diamond \diamond} \theta, z ; t\right)+\mathcal{R}_{2}\left(\dot{Z}, \pi^{\diamond} \theta, \pi^{\diamond \diamond} \theta ; t\right)+\mathcal{R}_{3}\left(\dot{\theta}, \pi^{\diamond} \theta ; t\right) \\
& \quad+\mathcal{R}_{4}\left(\pi^{\diamond} \dot{\theta}, \pi^{\diamond} \theta ; t\right)+\mathcal{R}_{5}\left(\pi^{\diamond} \theta, \pi^{\diamond \diamond} \theta, \pi^{\diamond \diamond \diamond} \theta ; t\right) \\
&+\mathcal{E}_{1}\left(\pi^{\diamond \diamond} \dot{\theta} ; t\right)+\mathcal{E}_{2}\left(\pi^{\diamond \diamond \diamond} \theta ; t\right) .
\end{aligned}
$$

We emphasize at this point that in the sequel all the terms contained in numbered expressions will be bounded by the terms that are kept in their explicit form.

Our task is to choose the inhomogeneities (5.110) in such a way that all the explicit terms in (5.123) that do not involve the function $z$ are of the form $\mathrm{J}\left(*, \Phi^{\prime} ; t\right)$. This way, the dependence of these terms on $\xi_{n l}(t)$ can be factored out, leaving only terms that merely depend on the transverse coordinate $l$. To this end, we start by writing

$$
f_{p ; \nu}^{\diamond}(\xi)=\left[\tau_{\nu} \Phi^{\prime}\right](\xi)-\alpha_{p ; \nu}^{\diamond} \Phi^{\prime}(\xi),
$$

in which the choice

$$
\alpha_{p ; \nu}^{\diamond}=\int_{\mathbb{R}} \Psi(\xi)\left[\tau_{\nu} \Phi^{\prime}\right](\xi) d \xi,
$$

ensures by the characterization (2.19) that one can find functions $p_{\nu}^{\diamond} \in B C^{1}(\mathbb{R}, \mathbb{R})$ for which the relevant identity in $(5.110)$ holds.

Incorporating the definitions above into (5.123), we arrive at the identity

$$
\begin{aligned}
& \mathcal{J}^{-}(t)=-\mathrm{J}\left(\dot{Z}, \Phi^{\prime} ; t\right)-\dot{z}(t)+\mathrm{J}(L ; t) z(t)-\mathrm{J}\left(\dot{\theta}, \Phi^{\prime} ; t\right)+\mathrm{J}\left(\alpha_{p ; \mu}^{\diamond} \pi_{\mu}^{\diamond} \theta, \Phi^{\prime} ; t\right) \\
&+\mathrm{J}\left(\alpha_{p ; \mu}^{\diamond} \pi_{; \mu \mu^{\prime}}^{\diamond} \theta, p_{\mu^{\prime}}^{\diamond} ; t\right)-\mathrm{J}\left(\pi_{; \mu \mu^{\prime}}^{\diamond} \theta, \tau_{\mu^{\prime}} p_{\mu}^{\diamond} ; t\right)-\mathrm{J}\left(\pi_{; \mu \mu^{\prime}}^{\diamond \diamond} \theta, f_{p ; \mu \mu^{\prime}}^{\diamond \diamond} ;\right) \\
&-\mathrm{J}\left(\alpha_{p ; \mu}^{\diamond} \pi_{\mu}^{\diamond} \theta, \pi_{; \mu^{\prime}}^{\diamond} \theta, D p_{\mu^{\prime}}^{\diamond} ; t\right)+\mathrm{J}\left(\pi_{; \mu}^{\diamond} \theta, \pi_{; \mu^{\prime}}^{\diamond} \theta, \tau_{\mu^{\prime}} D p_{\mu}^{\diamond} ; t\right)-\mathrm{J}\left(\pi_{; \mu}^{\diamond} \theta, \pi_{; \mu^{\prime}}^{\diamond} \theta, f_{q ; \mu \mu^{\prime}}^{\diamond \diamond}, t\right) \\
&+\mathcal{R}_{1}\left(\pi^{\diamond} \theta, \pi^{\diamond} \theta, z ; t\right)+\mathcal{R}_{2}\left(\dot{Z}, \pi^{\diamond} \theta, \pi^{\diamond} \theta ; t\right)+\mathcal{R}_{3}\left(\dot{\theta}, \pi^{\diamond \diamond} \theta ; t\right) \\
& \quad+\mathcal{R}_{4}\left(\pi^{\diamond} \dot{\theta}, \pi^{\diamond} \theta ; t\right)+\mathcal{R}_{5}\left(\pi^{\diamond} \theta, \pi^{\diamond \diamond} \theta, \pi^{\diamond \diamond} \theta ; t\right)+\mathcal{R}_{6}\left(\dot{\theta}, \pi^{\diamond} \theta ; t\right) \\
&+\mathcal{E}_{1}\left(\pi^{\diamond \diamond} \dot{\theta} ; t\right)+\mathcal{E}_{2}\left(\pi^{\diamond \diamond \diamond} \theta ; t\right)+\mathcal{E}_{3}\left(\pi^{\diamond} \dot{\theta}, \pi^{\diamond \diamond} \theta ; t\right),
\end{aligned}
$$

in which we have introduced the extra nonlinear expression

$$
\mathcal{R}_{6}\left(\dot{\theta}, \pi^{\diamond} \theta ; t\right)={ }_{\mathrm{J}} \mathrm{J}\left(\dot{\theta}-\alpha_{\mu}^{\diamond} \pi_{\mu}^{\diamond} \theta, \pi_{; \mu^{\prime}}^{\diamond} \theta, D p_{\mu^{\prime}}^{\diamond} ; t\right),
$$

together with the extra linear expression

$$
\mathcal{E}_{3}\left(\pi^{\diamond} \dot{\theta}, \pi^{\diamond} \theta ; t\right)=\mathrm{J}\left(\pi_{; \mu^{\prime}}^{\diamond}\left[\dot{\theta}-\alpha_{\mu}^{\diamond} \pi_{\mu}^{\diamond} \theta\right], p_{\mu^{\prime}}^{\diamond} ; t\right) .
$$

In order to satisfy the goal mentioned above, it now suffices to pick

$$
\begin{aligned}
& f_{p ; \nu \nu^{\prime}}^{\diamond}(\xi)=\alpha_{p ; \nu}^{\diamond} p_{\nu^{\prime}}^{\diamond}(\xi)-\left[\tau_{\nu^{\prime}} p_{\nu}^{\diamond}\right](\xi)-\alpha_{p ; \nu \nu^{\prime}}^{\diamond \diamond} \Phi^{\prime}(\xi), \\
& f_{q ; \nu \nu^{\prime}}^{\diamond \diamond}(\xi)=-\alpha_{p ; \nu}^{\diamond} D p_{\nu^{\prime}}^{\diamond}(\xi)+\left[\tau_{\nu^{\prime}} D p_{\nu}^{\diamond}\right](\xi)-\alpha_{q ; \nu \nu^{\prime}}^{\diamond \diamond} \Phi^{\prime}(\xi),
\end{aligned}
$$


in which we have introduced the constants

$$
\begin{aligned}
\alpha_{p ; \nu \nu^{\prime}}^{\diamond \diamond} & =\int_{\mathbb{R}} \Psi(\xi)\left[\alpha_{p ; \nu}^{\diamond} p_{\nu^{\prime}}^{\diamond}(\xi)-\left[\tau_{\nu^{\prime}} p_{\nu}^{\diamond}\right](\xi)\right] d \xi \\
\alpha_{q ; \nu \nu^{\prime}}^{\diamond \diamond} & =\int_{\mathbb{R}} \Psi(\xi)\left[-\alpha_{p ; \nu}^{\diamond} D p_{\nu^{\prime}}^{\diamond}(\xi)+\left[\tau_{\nu^{\prime}} D p_{\nu}^{\diamond}\right](\xi)\right] d \xi .
\end{aligned}
$$

As above, these choices ensure that one can find functions $p_{\nu \nu^{\prime}}^{\diamond} q_{\nu \nu^{\prime}}^{\diamond} \in B C^{1}(\mathbb{R}, \mathbb{R})$ for which the relevant identities in (5.110) hold.

Upon introducing our final nonlinear expression

$$
\mathcal{R}_{7}\left(\pi^{\diamond} \theta ; t\right)=\alpha_{q ; \mu \mu^{\prime}}^{\diamond} \mathrm{J}\left(\pi_{; \mu}^{\diamond} \theta, \pi_{; \mu^{\prime}}^{\diamond} \theta, \Phi^{\prime} ; t\right),
$$

we can summarize our computations in the following result.

Lemma 5.3. Pick any pair $\left(\sigma_{h}, \sigma_{v}\right) \in \mathbb{Z}^{2} \backslash\{(0,0)\}$ and suppose that $(H g)$ and $(h \Phi)_{\S 3}$ both hold. Then for every $\left(\nu, \nu^{\prime}\right) \in\{1, \ldots 5\}^{2}$, there exist functions

$$
p_{\nu}^{\diamond}, p_{\nu \nu^{\prime}}^{\diamond \diamond}, q_{\nu \nu^{\prime}}^{\diamond \diamond} \in B C^{2}(\mathbb{R}, \mathbb{R})
$$

that satisfy the identities

$$
\begin{aligned}
& {\left[\mathcal{L}_{0} p_{\nu}^{\diamond}\right](\xi)=\left[\tau_{\nu} \Phi^{\prime}\right](\xi)-\alpha_{p ; \nu}^{\diamond} \Phi^{\prime}(\xi)} \\
& {\left[\mathcal{L}_{0} p_{\nu \nu^{\prime}}^{\diamond \diamond}\right](\xi)=\alpha_{p ; \nu}^{\diamond} p_{\nu^{\prime}}^{\diamond}(\xi)-\left[\tau_{\nu^{\prime}} p_{\nu}^{\diamond}\right](\xi)-\alpha_{p ; \nu \nu^{\prime}}^{\diamond \diamond} \Phi^{\prime}(\xi),} \\
& {\left[\mathcal{L}_{0} q_{\nu \nu^{\prime}}^{\diamond \diamond}\right](\xi)=-\alpha_{p ; \nu}^{\diamond} D p_{\nu^{\prime}}^{\diamond}(\xi)+\left[\tau_{\nu^{\prime}} D p_{\nu}^{\diamond}\right](\xi)-\alpha_{q ; \nu \nu^{\prime}}^{\diamond} \Phi^{\prime}(\xi),}
\end{aligned}
$$

with coefficients

$$
\begin{aligned}
& \alpha_{p, \nu}^{\diamond}=\int_{\mathbb{R}} \Psi(\xi)\left[\tau_{\nu} \Phi^{\prime}\right](\xi) d \xi, \\
& \alpha_{p ; \nu \nu^{\prime}}^{\diamond \diamond}=\int_{\mathbb{R}} \Psi(\xi)\left[\alpha_{p ; \nu}^{\diamond} p_{\nu^{\prime}}^{\diamond}(\xi)-\left[\tau_{\nu^{\prime}} p_{\nu}^{\diamond}\right](\xi)\right] d \xi, \\
& \alpha_{q ; \nu \nu^{\prime}}^{\diamond}=\int_{\mathbb{R}} \Psi(\xi)\left[-\alpha_{p ; \nu}^{\diamond} D p_{\nu^{\prime}}^{\diamond}(\xi)+\left[\tau_{\nu^{\prime}} D p_{\nu}^{\diamond}\right](\xi)\right] d \xi .
\end{aligned}
$$

In addition, for every triplet of $C^{1}$-smooth functions

$$
z:[0, \infty) \rightarrow \mathbb{R}, \quad Z:[0, \infty) \rightarrow \mathbb{R}, \quad \theta:[0, \infty) \rightarrow \ell^{\infty}(\mathbb{Z} ; \mathbb{R}),
$$

the function $u^{-}:[0, \infty) \rightarrow \ell^{\infty}\left(\mathbb{Z}^{2} ; \mathbb{R}\right)$, defined by

$$
u^{-}(t)=\mathrm{J}(\Phi ; t)+\mathrm{J}\left(\pi_{; \mu}^{\diamond} \theta, p_{\mu}^{\diamond} ; t\right)+\mathrm{J}\left(\pi_{; \mu \mu^{\prime}}^{\diamond \diamond} \theta, p_{\mu \mu^{\prime}}^{\diamond \diamond} ; t\right)+\mathrm{J}\left(\pi_{; \mu}^{\diamond} \theta, \pi_{; \mu^{\prime}}^{\diamond} \theta, q_{\mu \mu^{\prime}}^{\diamond \diamond} ; t\right)-z(t)
$$

with $\xi_{n l}(t)=n+c t-\theta_{l}(t)-Z(t)$, admits the identity

$$
\begin{aligned}
& \mathcal{J}^{-}(t):=\quad \dot{u}^{-}(t)-\Delta^{\times} u^{-}(t)-g\left(u^{-}(t)\right) \\
&=\quad-\mathrm{J}(\dot{Z},\left.\Phi^{\prime} ; t\right)-\dot{z}(t)+\mathrm{J}(L ; t) z(t) \\
&-\mathrm{J}\left(\dot{\theta}, \Phi^{\prime} ; t\right)+\alpha_{p ; \mu \mathrm{J}}^{\diamond}\left(\pi_{\mu}^{\diamond} \theta, \Phi^{\prime} ; t\right)+\alpha_{p ; \mu \mu^{\prime} \mathrm{J}}^{\diamond \diamond}\left(\pi_{\mu \mu^{\prime}}^{\diamond \diamond} \theta, \Phi^{\prime} ; t\right) \\
&+\mathcal{R}_{1}\left(\pi^{\diamond} \theta, \pi^{\diamond \diamond} \theta, z ; t\right)+\mathcal{R}_{2}\left(\dot{Z}, \pi^{\diamond} \theta, \pi^{\diamond} \theta ; t\right)+\mathcal{R}_{3}\left(\dot{\theta}, \pi^{\diamond \diamond} \theta ; t\right) \\
& \quad+\mathcal{R}_{4}\left(\pi^{\diamond} \dot{\theta}, \pi^{\diamond} \theta ; t\right)+\mathcal{R}_{5}\left(\pi^{\diamond} \theta, \pi^{\diamond \diamond} \theta, \pi^{\diamond \diamond} \theta ; t\right)+\mathcal{R}_{6}\left(\dot{\theta}, \pi^{\diamond} \theta ; t\right)+\mathcal{R}_{7}\left(\pi^{\diamond} \theta ; t\right) \\
&+\mathcal{E}_{1}\left(\pi^{\diamond \diamond} \dot{\theta} ; t\right)+\mathcal{E}_{2}\left(\pi^{\diamond \diamond \diamond} \theta ; t\right)+\mathcal{E}_{3}\left(\pi^{\diamond} \dot{\theta}, \pi^{\diamond \diamond} \theta ; t\right) .
\end{aligned}
$$

Here the nonlinear expressions $\mathcal{R}_{1}$ through $\mathcal{R}_{7}$ are defined in (5.119), (5.120), (5.121), (5.127) and (5.131), while the linear expressions $\mathcal{E}_{1}$ through $\mathcal{E}_{3}$ are defined in (5.122) and (5.128).

Finally, for any $\nu \in\{1, \ldots 4\}$ we have

$$
\left[\pi_{; \nu}^{\times}-\pi_{; 5}^{\times}\right] u^{-}(t)=\mathcal{R}_{\mathcal{N} ; \nu}\left(\pi^{\diamond} \theta, \pi^{\diamond \diamond} \theta ; t\right),
$$

with $\mathcal{R}_{\mathcal{N} ; \nu}$ defined in (5.101). 
In the remainder of this subsection we derive some useful estimates on the numbered terms appearing in (5.137). In addition, we establish a critical relation between the expressions (5.133)(5.134) and spectral properties of the operators $\mathcal{L}_{\omega}$ discussed in Proposition 2.1.

Lemma 5.4. Consider the setting of Lemma 5.3. There exist constants $K_{\diamond}>1$ and $\eta_{\diamond}>0$ such that we have the bounds

$$
\begin{aligned}
\left|p^{\diamond}(\xi)\right|+\left|p^{\diamond \diamond}(\xi)\right|+\left|q^{\diamond \diamond}(\xi)\right| & \leq K_{\diamond} e^{-\eta_{\diamond}|\xi|}, \\
\left|D p^{\diamond}(\xi)\right|+\left|D p^{\diamond \diamond}(\xi)\right|+\left|D q^{\diamond \diamond}(\xi)\right| & \leq K_{\diamond} e^{-\eta_{\diamond}|\xi|},
\end{aligned}
$$

for all $\xi \in \mathbb{R}$. In addition, we have the bounds

$$
\left[\left|p^{\diamond}(\xi)\right|+\left|p^{\diamond \diamond}(\xi)\right|+\left|q^{\diamond \diamond}(\xi)\right|\right]^{2} \leq K_{\diamond} \Phi^{\prime}(\xi)
$$

again for all $\xi \in \mathbb{R}$.

Proof. Upon recalling the constants $\eta_{\Phi}^{ \pm}$from Lemma 3.3, let us write $\eta_{\diamond}^{ \pm}=\frac{3}{4} \eta_{\Phi}^{ \pm}$. Upon writing

$$
\Lambda_{\text {inv }}^{+}: B C_{-\eta_{\diamond}^{+}}([0, \infty), \mathbb{R}) \rightarrow B C_{-\eta_{\diamond}^{+}}^{1}([-\sigma, \infty), \mathbb{R})
$$

for the inverse of $\mathcal{L}_{0}$ that has properties analogous to those stated in Lemma 3.11, we can write

$$
\left[p_{\nu}^{\diamond}\right]_{\mid[-\sigma, \infty)}=\Lambda_{\text {inv }}^{+}\left[\tau_{\nu} \Phi^{\prime}-\alpha_{p ; \nu}^{\diamond} \Phi^{\prime}\right] .
$$

Similar properties hold on $(-\infty, \sigma]$ and the desired estimates now follow directly from these observations.

Lemma 5.5. Consider the setting of Lemma 5.3. We have the identities

$$
\begin{aligned}
& {\left[\frac{d}{d \omega} \lambda_{\omega}\right]_{\omega=0}=i \sigma_{\mu} \alpha_{\mu}^{\diamond},} \\
& {\left[\frac{d^{2}}{d \omega^{2}} \lambda_{\omega}\right]_{\omega=0}=-\sigma_{\mu}^{2} \alpha_{p ; \mu}^{\diamond}-2 \sigma_{\mu} \sigma_{\mu^{\prime}} \alpha_{p ; \mu \mu^{\prime}}^{\diamond} .}
\end{aligned}
$$

Proof. Throughout this proof we use the shorthand $D_{\omega}=\frac{d}{d \omega}$. Taylor expanding the quantities $\lambda_{\omega}$ and $\phi_{\omega}$ for $\omega \approx 0$ in the expression $\left(\mathcal{L}_{\omega}-\lambda_{\omega}\right) \phi_{\omega}=0$ and remembering that $\phi_{0}=\Phi^{\prime}$, we obtain

$$
\begin{aligned}
O\left(\omega^{3}\right)=\mathcal{L}_{0} \Phi^{\prime} & +\omega\left(\mathcal{L}_{0}\left[D_{\omega} \phi_{\omega}\right]_{\omega=0}+\left[D_{\omega} \mathcal{L}_{\omega}-D_{\omega} \lambda_{\omega}\right]_{\omega=0} \Phi^{\prime}\right) \\
+ & \frac{\omega^{2}}{2}\left(\mathcal{L}_{0}\left[D_{\omega}^{2} \phi_{\omega}\right]_{\omega=0}+2\left[D_{\omega} \mathcal{L}_{\omega}-D_{\omega} \lambda_{\omega}\right]_{\omega=0}\left[D_{\omega} \phi_{\omega}\right]_{\omega=0}+\left[D_{\omega}^{2} \mathcal{L}_{\omega}-D_{\omega}^{2} \lambda_{\omega}\right]_{\omega=0} \Phi^{\prime}\right) .
\end{aligned}
$$

Taking the inner product against $\Psi$ and recalling that $\mathcal{L}_{0}^{*} \Psi=0$ leaves

$$
\left[D_{\omega} \lambda_{\omega}\right]_{\omega=0}=\int_{-\infty}^{\infty} \Psi(\xi)\left[\left[D_{\omega} \mathcal{L}_{\omega}\right]_{\omega=0} \Phi^{\prime}\right](\xi) d \xi
$$

Recalling the identity

$$
\left[\mathcal{L}_{\omega} v\right](\xi)=-c v^{\prime}(\xi)+L_{\mu}^{\times} \exp \left(i \sigma_{\mu} \omega\right)\left[\tau_{\mu} v\right](\xi)+g^{\prime}(\Phi(\xi)) v
$$

we may compute

$$
\begin{aligned}
{\left[D_{\omega} \mathcal{L}_{\omega} v\right](\xi) } & =i \sigma_{\mu} L_{\mu}^{\times} \exp \left(i \sigma_{\mu} \omega\right)\left[\tau_{\mu} v\right](\xi) \\
& =i \sigma_{\mu} \exp \left(i \sigma_{\mu} \omega\right)\left[\tau_{\mu} v\right](\xi) \\
{\left[D_{\omega}^{2} \mathcal{L}_{\omega} v\right](\xi) } & =-\sigma_{\mu}^{2} \exp \left(i \sigma_{\mu} \omega\right)\left[\tau_{\mu} v\right](\xi)
\end{aligned}
$$


We hence see that

$$
\left[D_{\omega} \mathcal{L}_{\omega}\right]_{\omega=0} \Phi^{\prime}=i \sigma_{\mu} \tau_{\mu} \Phi^{\prime}
$$

which implies

$$
\begin{aligned}
{\left[D_{\omega} \lambda_{\omega}\right]_{\omega=0} } & =i \sigma_{\mu} \int_{-\infty}^{\infty} \Psi(\xi)\left[\tau_{\mu} \Phi^{\prime}\right](\xi) d \xi \\
& =i \sigma_{\mu} \alpha_{\mu}^{\diamond}
\end{aligned}
$$

as desired.

Moving on, we see that

$$
\begin{aligned}
\mathcal{L}_{0}\left[D_{\omega} \phi_{\omega}\right]_{\omega=0} & =i \sigma_{\mu} \alpha_{\mu}^{\diamond} \Phi^{\prime}-i \sigma_{\mu} \tau_{\mu} \Phi^{\prime} \\
& =-i \sigma_{\mu} f_{\mu}^{\diamond} .
\end{aligned}
$$

In particular, up to multiples of $\Phi^{\prime}$ we may write

$$
\left[D_{\omega} \phi_{\omega}\right]_{\omega=0}=-i \sigma_{\mu} p_{\mu}^{\diamond}
$$

We now integrate the $O\left(\omega^{2}\right)$ term in (5.144) against $\Psi$, yielding

$$
\begin{aligned}
{\left[D_{\omega}^{2} \lambda_{\omega}\right]_{\omega=0}=\int_{-\infty}^{\infty} \Psi(\xi)\left[\left[D_{\omega}^{2} \mathcal{L}_{\omega}\right]_{\omega=0} \Phi^{\prime}\right](\xi) d \xi } \\
+2 \int_{-\infty}^{\infty} \Psi(\xi)\left[\left[D_{\omega} \mathcal{L}_{\omega}-D_{\omega} \lambda_{\omega}\right]_{\omega=0}\left[D_{\omega} \phi_{\omega}\right]_{\omega=0}\right](\xi) d \xi
\end{aligned}
$$

Plugging in the identities obtained above, we obtain

$$
\begin{aligned}
{\left[D_{\omega}^{2} \lambda_{\omega}\right]_{\omega=0}=} & \int_{-\infty}^{\infty} \Psi(\xi)\left[-\sigma_{\mu}^{2} \tau_{\mu} \Phi^{\prime}\right](\xi) d \xi \\
& \quad+2 \int_{-\infty}^{\infty} \Psi(\xi)\left[\left[i \sigma_{\mu} \tau_{\mu}-i \sigma_{\mu} \alpha_{\mu}^{\diamond}\right]\left[-i \sigma_{\mu^{\prime}} p_{\mu^{\prime}}^{\diamond}\right]\right](\xi) d \xi \\
= & -\sigma_{\mu}^{2} \alpha_{\mu}^{\diamond}+2 \sigma_{\mu} \sigma_{\mu^{\prime}} \int_{-\infty}^{\infty} \Psi(\xi)\left[\tau_{\mu} p_{\mu^{\prime}}^{\diamond}-\alpha_{\mu}^{\diamond} p_{\mu^{\prime}}\right](\xi) d \xi \\
= & -\sigma_{\mu}^{2} \alpha_{\mu}^{\diamond}+2 \sigma_{\mu} \sigma_{\mu^{\prime}} \int_{-\infty}^{\infty} \Psi(\xi)\left[\tau_{\mu^{\prime}} p_{\mu}^{\diamond}-\alpha_{\mu}^{\diamond} p_{\mu^{\prime}}^{\diamond}\right](\xi) d \xi \\
= & -\sigma_{\mu}^{2} \alpha_{\mu}^{\diamond}-2 \sigma_{\mu} \sigma_{\mu^{\prime}} \alpha_{\mu \mu^{\prime}}^{\diamond}
\end{aligned}
$$

as desired.

Lemma 5.6. Fix any pair $\left(\sigma_{h}, \sigma_{v}\right) \in \mathbb{Z}^{2} \backslash\{(0,0)\}$, suppose that $(H g)$ and $(h \Phi)_{\S 3}$ both hold and pick any $M_{1}>0$. Then there exists a constant $C_{1}=C_{1}\left(M_{1}\right)>1$ such that for every pair of $C^{1}$-smooth functions

$$
\theta:[0, \infty) \rightarrow \ell^{\infty}(\mathbb{Z} ; \mathbb{R}), \quad z:[0, \infty) \rightarrow \mathbb{R}
$$

that satisfy the bound

$$
|z(t)|+\left\|\pi^{\diamond} \theta(t)\right\|_{\ell^{\infty}\left(\mathbb{Z} ; \mathbb{R}^{5}\right)}<M_{1}, \quad t \geq 0
$$

we have the estimates

$$
\begin{aligned}
\left|\left[\mathcal{R}_{1}\left(\pi^{\diamond} \theta, \pi^{\diamond \diamond} \theta, z ; t\right)\right]_{n l}\right| \leq & C_{1}|z(t)|\left(|z(t)|+\left|\pi_{l}^{\diamond} \theta(t)\right|+\left|\pi_{l}^{\diamond \diamond} \theta(t)\right|\right) \\
& +C_{1}\left(\left|\pi_{l}^{\diamond} \theta(t)\right|+\left|\pi_{l}^{\diamond \diamond} \theta(t)\right|\right)^{2} \Phi^{\prime}\left(\xi_{n l}(t)\right), \\
\left|\left[\mathcal{R}_{7}\left(\pi^{\diamond} \theta ; t\right)\right]_{n l}\right| \leq & C_{1}\left|\pi_{l}^{\diamond} \theta(t)\right|^{2} \Phi^{\prime}\left(\xi_{n l}(t)\right)
\end{aligned}
$$

for every $(n, l) \in \mathbb{Z}^{2}$ and $t \geq 0$. 
Proof. On account of the a-priori bound $\left|\pi_{l}^{\diamond} \theta\right|<M_{1}$, we can invoke Corollary 3.6 to obtain

$$
\left|\mathcal{M}_{\Phi, 2, \mu}\left(\xi_{n l}(t), \pi_{l ; \mu}^{\diamond} \theta(t)\right)\right| \leq C_{1}^{\prime} \Phi^{\prime}\left(\xi_{n l}(t)\right)\left|\pi_{l ; \mu}^{\diamond} \theta(t)\right|^{2}
$$

for some $C_{1}^{\prime}=C_{1}^{\prime}\left(M_{1}\right)>1$. In addition, the a-priori bound on $u^{-}(t)-\mathrm{J}(\Phi ; t)$ allows us to write

$$
\left|\left[\mathcal{G}_{0}\left(u^{-}(t)-\mathrm{J}(\Phi ; t)\right)\right]_{n l}\right| \leq C_{2}^{\prime}\left|u_{n l}^{-}(t)-\mathrm{J}_{n l}(\Phi ; t)\right|^{2}
$$

for some $C_{2}^{\prime}>0$. The desired estimates follow directly from these observations upon utilizing the bound (5.140).

Lemma 5.7. Fix any pair $\left(\sigma_{h}, \sigma_{v}\right) \in \mathbb{Z}^{2} \backslash\{(0,0)\}$, suppose that $(\mathrm{Hg})$ and $(h \Phi)_{\S 3}$ both hold and pick any $M_{1}>0$. Then there exists a constant $C_{1}=C_{1}\left(M_{1}\right)>1$ such that for every pair of $C^{1}$-smooth functions

$$
\theta:[0, \infty) \rightarrow \ell^{\infty}(\mathbb{Z} ; \mathbb{R}), \quad Z:[0, \infty) \rightarrow \mathbb{R}
$$

that satisfy the bound

$$
\left\|\pi^{\diamond} \theta(t)\right\|_{\ell^{\infty}\left(\mathbb{Z} ; \mathbb{R}^{5}\right)}<M_{1}, \quad t \geq 0
$$

we have the estimates

$$
\begin{aligned}
& \left|\left[\mathcal{R}_{2}\left(\dot{Z}, \pi^{\diamond} \theta, \pi^{\diamond} \theta ; t\right)\right]_{n l}\right| \leq C_{1}|\dot{Z}(t)|\left[\left|\pi_{l}^{\diamond} \theta(t)\right|+\left|\pi_{l}^{\diamond} \theta(t)\right|\right], \\
& \left|\left[\mathcal{R}_{3}\left(\dot{\theta}, \pi^{\diamond \diamond} \theta ; t\right)\right]_{n l}\right| \leq C_{1}\left|\dot{\theta}_{l}(t)\right|\left|\pi_{l}^{\diamond \diamond} \theta(t)\right|, \\
& \left|\left[\mathcal{R}_{4}\left(\pi^{\diamond} \dot{\theta}, \pi^{\diamond} \theta ; t\right)\right]_{n l}\right| \leq C_{1}\left|\pi_{l}^{\diamond} \dot{\theta}(t)\right|\left|\pi_{l}^{\diamond} \theta(t)\right|, \\
& \left|\left[\mathcal{R}_{5}\left(\pi^{\diamond} \theta, \pi^{\diamond \diamond} \theta, \pi^{\diamond \diamond \diamond} \theta ; t\right)\right]_{n l}\right| \leq\left|\pi_{l}^{\diamond} \theta(t)\right|\left[\left|\pi_{l}^{\diamond \diamond} \theta(t)\right|+\left|\pi_{l}^{\diamond} \theta(t)\right|^{2}+\left|\pi_{l}^{\diamond \diamond \diamond} \theta(t)\right|\right] \\
& +\left|\pi_{l}^{\diamond \diamond} \theta(t)\right|^{2} \\
& \left|\left[\mathcal{R}_{6}\left(\dot{\theta}, \pi^{\diamond} \theta ; t\right)\right]_{n l}\right| \leq C_{1}\left|\dot{\theta}_{l}(t)-\alpha_{\mu}^{\diamond} \pi_{l ; \mu}^{\diamond} \theta(t)\right|\left|\pi_{l}^{\diamond} \theta(t)\right|, \\
& \left|\left[\mathcal{R}_{\mathcal{N} ; \nu}\left(\pi^{\diamond} \theta, \pi^{\diamond \diamond} \theta, \pi^{\diamond \diamond \diamond} \theta ; t\right)\right]_{n l}\right| \leq C_{1} e^{-\kappa_{\diamond}\left|\xi_{n l}(t)\right|}
\end{aligned}
$$

for every $(n, l) \in \mathbb{Z}^{2}$ and $t \geq 0$.

Proof. These bounds follow directly from the definitions (5.101), (5.119), (5.120), (5.121), (5.127) and (5.131), together with the bounds (5.139).

Lemma 5.8. Fix any pair $\left(\sigma_{h}, \sigma_{v}\right) \in \mathbb{Z}^{2} \backslash\{(0,0)\}$ and suppose that $(H g)$ and $(h \Phi)_{\S 3}$ both hold. Then there exists a constant $C_{1}>1$ such that for every $C^{1}$-smooth function

$$
\theta:[0, \infty) \rightarrow \ell^{\infty}(\mathbb{Z} ; \mathbb{R}),
$$

we have the bounds

$$
\begin{aligned}
&\left|\left[\mathcal{E}_{1}\left(\pi^{\diamond \diamond} \dot{\theta} ; t\right)\right]_{n l}\right| \leq C_{1}\left|\pi_{l}^{\diamond \diamond} \dot{\theta}(t)\right|, \\
&\left|\left[\mathcal{E}_{2}\left(\pi^{\diamond \diamond \diamond} \theta ; t\right)\right]_{n l}\right| \leq C_{1}\left|\pi_{l}^{\diamond \diamond \diamond} \theta(t)\right|, \\
&\left|\left[\mathcal{E}_{3}\left(\pi^{\diamond} \dot{\theta}, \pi^{\diamond \diamond} \theta ; t\right)\right]_{n l}\right| \leq C_{1}\left|\pi_{l}^{\diamond}\left[\dot{\theta}(t)-\alpha_{\mu}^{\diamond} \pi_{l ; \mu}^{\diamond} \theta(t)\right]\right|
\end{aligned}
$$

for all $(n, l) \in \mathbb{Z}^{2}$ and $t \geq 0$.

Proof. These estimates follow directly from the definitions (5.122) and (5.128) together with the bounds (5.139). 


\subsection{The expanding plateau}

In this subsection we construct a function $v:[1, \infty) \rightarrow \ell^{\infty}(\mathbb{Z} ; \mathbb{R})$ that can be thought of as an expanding and convecting plateau. Later on, we will obtain the function $\theta$ by multiplying $v$ by a global prefactor that decays in time.

For convenience, we define the quantities

$$
\begin{aligned}
\nu_{1} & =\frac{1}{i}\left[\frac{d}{d \omega} \lambda_{\omega}\right]_{\omega=0}, \\
\nu_{2} & =-\frac{1}{2}\left[\frac{d^{2}}{d \omega^{2}} \lambda_{\omega}\right]_{\omega=0} .
\end{aligned}
$$

We assume throughout this section that $\nu_{2}>0$, noting that this is a consequence of the Melnikov condition $(H S)_{\zeta_{*}}$. For any $\gamma \geq 1$ and $t \geq 1$, we define

$$
v_{l ; \gamma}(t)=(\gamma t)^{1 / 2} \int_{-\infty}^{\infty} \exp \left[i \omega\left(l+\nu_{1} t\right)\right] \exp \left[-\nu_{2} \omega^{2} \gamma t\right] d \omega,
$$

which can be explicitly evaluated as

$$
v_{l ; \gamma}(t)=\sqrt{\frac{\pi}{\nu_{2}}} \exp \left[-\frac{\left(l+\nu_{1} t\right)^{2}}{4 \nu_{2} \gamma t}\right]
$$

We also introduce the functions

$$
\mathcal{V}_{l ; \gamma}^{(k)}(t)=(\gamma t)^{1 / 2} \int_{-\infty}^{\infty} \omega^{k} \exp \left[i \omega\left(l+\nu_{1} t\right)\right] \exp \left[-\nu_{2} \omega^{2} \gamma t\right] d \omega
$$

for integers $k \geq 1$, which can be evaluated as

$$
\begin{aligned}
\mathcal{V}_{l ; \gamma}^{(1)}(t) & =\frac{1}{2} i \frac{l+\nu_{1} t}{\nu_{2} \gamma t} v_{l ; \gamma}(t) \\
\mathcal{V}_{l ; \gamma}^{(2)}(t) & =\left[-\frac{1}{4} \frac{\left(l+\nu_{1} t\right)^{2}}{\left(\nu_{2} \gamma t\right)^{2}}+\frac{1}{2} \frac{1}{\nu_{2} \gamma t}\right] v_{l ; \gamma}(t) \\
\mathcal{V}_{l ; \gamma}^{(3)}(t) & =\left[-\frac{i}{8} \frac{\left(l+\nu_{1} t\right)^{3}}{\left(\nu_{2} \gamma t\right)^{3}}+\frac{3 i}{4} \frac{\left(l+\nu_{1} t\right)}{\left(\nu_{2} \gamma t\right)}\left(\nu_{2} \gamma t\right)^{-1}\right] v_{l ; \gamma}(t)
\end{aligned}
$$

To prevent cumbersome notation, we introduce the shorthand

$$
\rho=\rho(l, t ; \gamma)=\frac{l+\nu_{1} t}{2 \nu_{2} \gamma t},
$$

which allows us to reduce the expressions above to the compact form

$$
\begin{aligned}
\mathcal{V}_{l ; \gamma}^{(1)}(t) & =i \rho v_{l ; \gamma}(t) \\
\mathcal{V}_{l ; \gamma}^{(2)}(t) & =\left[-\rho^{2}+\frac{1}{2}\left(\nu_{2} \gamma t\right)^{-1}\right] v_{l ; \gamma}(t), \\
\mathcal{V}_{l ; \gamma}^{(3)}(t) & =\left[-i \rho^{3}+\frac{3 i}{2} \rho\left(\nu_{2} \gamma t\right)^{-1}\right] v_{l ; \gamma}(t) .
\end{aligned}
$$

Our first result studies the effects of non-polynomial Fourier multipliers applied to $v_{; \gamma}(t)$.

Lemma 5.9. Pick a sufficiently small $\delta_{\omega}>0$ and any $M>1$. Consider any analytic function $\wp: \mathbb{C} \rightarrow \mathbb{C}$ that has

$$
|\wp(\omega)| \leq M\left(1+\omega^{4}\right), \quad \omega \in \mathbb{R}
$$

and satisfies the bound

$$
|\wp(\omega)| \leq M|\omega|^{s}, \quad-\delta_{\omega} \leq \operatorname{Re} \omega \leq \delta_{\omega}, \quad-\delta_{\omega} \leq \operatorname{Im} \delta \leq+\delta_{\omega}
$$


for some $s \in\{0,2,4\}$. In addition, consider the expression

$$
\mathcal{W}_{l ; \gamma}^{(\wp)}(t)=(\gamma t)^{1 / 2} \int_{-\infty}^{\infty} \wp(\omega) \exp \left[i \omega\left(l+\nu_{1} t\right)\right] \exp \left[-\nu_{2} \omega^{2} \gamma t\right] d \omega .
$$

Then for any triplet $(l, t, \gamma)$ with $\gamma \geq 1, t \geq 1$ and $|\rho(l, t ; \gamma)| \leq \delta_{\omega}$, we have the bounds

$$
\left|\mathcal{W}_{l ; \gamma}^{(\wp)}(t)\right| \leq\left\{\begin{aligned}
M v_{l ; \gamma}(t) & \text { for } s=0, \\
+M\left[(\gamma t)^{1 / 2}+16 \nu_{2}^{-1} \delta_{\omega}^{-1}\left[\frac{3}{4}\left(\nu_{2} \delta_{\omega}\right)^{-4}+1\right]\right] e^{-\nu_{2} \delta_{\omega}^{2} \gamma t}, & \\
2 M v_{l ; \gamma}(t)\left[\rho^{2}+\frac{1}{2}\left(\nu_{2} \gamma t\right)^{-1}\right] & \text { for } s=2, \\
+M\left[(\gamma t)^{1 / 2}+16 \nu_{2}^{-1} \delta_{\omega}^{-1}\left[\frac{3}{4}\left(\nu_{2} \delta_{\omega}\right)^{-4}+1\right]\right] e^{-\nu_{2} \delta_{\omega}^{2} \gamma t}, & \\
8 M v_{l ; \gamma}(t)\left[\rho^{4}+\frac{3}{4}\left(\nu_{2} \gamma t\right)^{-2}\right] & \text { for } s=4, \\
+M\left[(\gamma t)^{1 / 2}+16 \nu_{2}^{-1} \delta_{\omega}^{-1}\left[\frac{3}{4}\left(\nu_{2} \delta_{\omega}\right)^{-4}+1\right]\right] e^{-\nu_{2} \delta_{\omega}^{2} \gamma t}, &
\end{aligned}\right.
$$

with $\rho=\rho(l, t ; \gamma)$. In addition, for any $(l, t, \gamma)$ with $\gamma \geq 1, t \geq 1$ and $|\rho(l, t ; \gamma)| \geq \delta_{\omega}$, we have

$$
\left|\mathcal{W}_{l ; \gamma}^{(\wp)}(t)\right| \leq M\left[2(\gamma t)^{1 / 2}+16 \nu_{2}^{-1} \delta_{\omega}^{-1}\left[\frac{3}{4}\left(\nu_{2} \delta_{\omega}\right)^{-4}+1\right]\right] e^{-\nu_{2} \delta_{\omega}^{2} \gamma t} .
$$

Proof. Upon introducing the expressions

$$
p(\omega)=-\nu_{2} \omega^{2} \gamma t, \quad q(\omega)=\omega\left(l+\nu_{1} t\right),
$$

we compute, for any $y \in \mathbb{R}$,

$$
\begin{aligned}
p(\omega+i y) & =-\nu_{2}\left(-y^{2}+2 i y \omega+\omega^{2}\right) \gamma t \\
& =\nu_{2} y^{2} \gamma t-i \omega y\left(2 \nu_{2} \gamma t\right)-\nu_{2} \omega^{2} \gamma t, \\
q(\omega+i y) & =i y\left(l+\nu_{1} t\right)+\omega\left(l+\nu_{1} t\right) \\
& =i y \rho\left(2 \nu_{2} \gamma t\right)+\omega \rho\left(2 \nu_{2} \gamma t\right)
\end{aligned}
$$

where $\rho=\rho(l, t ; \gamma)$. In other words, we have

$$
\operatorname{Re}[p(\omega+i y)+i q(\omega+i y)]=\left[y^{2}-2 y \rho\right] \nu_{2} \gamma t-\omega^{2} \nu_{2} \gamma t .
$$

For convenience, let us assume from now on that $\rho \geq 0$. On the interval $y \in[0, \rho]$, we have

$$
\operatorname{Re}[p(\omega+i y)+i q(\omega+i y)] \leq-\omega^{2} \nu_{2} \gamma t .
$$

For each fixed $\omega$, the expression (5.178) is minimized on the domain $y \in\left[0, \delta_{\omega}\right]$ upon choosing $y=y_{*}=\min \left\{\rho, \delta_{\omega}\right\}$. In the case $y_{*}=\rho$, we have

$$
p(\omega+i \rho)+i q(\omega+i \rho)=-\rho^{2} \nu_{2} \gamma t-\nu_{2} \omega^{2} \gamma t,
$$

while in the case $y_{*}=\delta_{\omega}$, we have

$$
\operatorname{Re}\left[p\left(\omega+i y_{*}\right)+i q\left(\omega+i y_{*}\right)\right] \leq-\nu_{2} \delta_{\omega}^{2} \gamma t-\omega^{2} \nu_{2} \gamma t .
$$

Upon introducing the five line segments

$$
\begin{aligned}
& \Gamma_{1}=\left(-\infty,-\delta_{\omega}\right], \quad \Gamma_{2}=\left[-\delta_{\omega},-\delta_{\omega}+i y_{*}\right], \quad \Gamma_{3}=\left[-\delta_{\omega}+i y_{*},+\delta_{\omega}+i y_{*}\right], \\
& \Gamma_{4}=\left[\delta_{\omega}+i y_{*},+\delta_{\omega}\right], \quad \Gamma_{5}=\left[\delta_{\omega}, \infty\right),
\end{aligned}
$$


we define the separate integrals

$$
\mathcal{W}_{l ; \gamma ; \Gamma_{i}}^{(\wp)}(t)=(\gamma t)^{1 / 2} \int_{\Gamma_{i}} \wp(\omega) \exp [p(\omega)+i q(\omega)] d \omega
$$

and note that Cauchy's theorem implies that

$$
\mathcal{W}_{l ; \gamma}^{(\wp)}(t)=\sum_{i=1}^{5} \mathcal{W}_{l ; \gamma ; \Gamma_{i}}^{(\wp)}(t) .
$$

Setting out to bound each of the integrals (5.183) separately, we start by computing

$$
\begin{aligned}
\int_{\delta_{\omega}}^{\infty}\left(1+\omega^{4}\right) e^{-\nu_{2} \omega^{2} \gamma t} d \omega & =e^{-\nu_{2} \delta_{\omega}^{2} \gamma t} \int_{\delta_{\omega}}^{\infty}\left(1+\omega^{4}\right) e^{-\nu_{2}\left(\omega^{2}-\delta_{\omega}^{2}\right) \gamma t} d \omega \\
& =e^{-\nu_{2} \delta_{\omega}^{2} \gamma t} \int_{\delta_{\omega}}^{\infty}\left(1+\omega^{4}\right) e^{-\nu_{2}\left(\omega-\delta_{\omega}\right)\left(\omega+\delta_{\omega}\right) \gamma t} d \omega \\
& \leq 8 e^{-\nu_{2} \delta_{\omega}^{2} \gamma t} \int_{\delta_{\omega}}^{\infty}\left[\left(\omega-\delta_{\omega}\right)^{4}+1+\delta_{\omega}^{4}\right] e^{-\nu_{2}\left(\omega-\delta_{\omega}\right) 2 \delta_{\omega} \gamma t} d \omega \\
& =8\left[24\left(2 \nu_{2} \delta_{\omega} \gamma t\right)^{-5}+\left(1+\delta_{\omega}^{4}\right)\left(2 \nu_{2} \delta_{\omega} \gamma t\right)^{-1}\right] e^{-\nu_{2} \delta_{\omega}^{2} \gamma t}
\end{aligned}
$$

In particular, imposing the restriction $0<\delta_{\omega} \leq \frac{1}{2}$ and remembering $\gamma t \geq 1$, we see that

$$
\left|\mathcal{W}_{l ; \gamma ; \Gamma_{1}}^{(\wp)}(t)\right|+\left|\mathcal{W}_{l ; \gamma ; \Gamma_{5}}^{(\wp)}(t)\right| \leq 16 M\left(\nu_{2} \delta_{\omega}\right)^{-1}\left[\frac{3}{4}\left(\nu_{2} \delta_{\omega}\right)^{-4}+1\right]
$$

Moving on, (5.179) implies that for all $\omega \in \Gamma_{2} \cup \Gamma_{4}$ we have

$$
\operatorname{Re}[p(\omega)+i q(\omega)] \leq-\nu_{2} \delta_{\omega}^{2} \gamma t
$$

Remembering that $0 \leq y_{*} \leq \delta_{\omega}$ and imposing the restriction $0<\delta_{\omega} \leq \frac{1}{2}$, we obtain

$$
\begin{aligned}
\left|\mathcal{W}_{l ; \gamma ; \Gamma_{2}}^{(m)}(t)\right|+\left|\mathcal{W}_{l ; \gamma ; \Gamma_{4}}^{(m)}(t)\right| & \leq 2 M \delta_{\omega}(\gamma t)^{1 / 2}\left|2 \delta_{\omega}\right|^{s} e^{-\nu_{2} \delta_{\omega}^{2} \gamma t} \\
& \leq M(\gamma t)^{1 / 2} e^{-\nu_{2} \delta_{\omega}^{2} \gamma t} .
\end{aligned}
$$

In addition, for $0 \leq \rho \leq \delta_{\omega}$ we have

$$
\begin{aligned}
(\gamma t)^{-1 / 2}\left|\mathcal{W}_{l ; \gamma ; \Gamma_{3}}^{(\wp)}(t)\right| & \leq \exp \left[-y_{*}^{2} \nu_{2} \gamma t\right] \int_{-\delta_{\omega}}^{\delta_{\omega}} M\left(|\omega|+\left|y_{*}\right|\right)^{s} e^{-\nu_{2} \omega^{2} \gamma t} d \omega \\
& \leq \exp \left[-y_{*}^{2} \nu_{2} \gamma t\right] \int_{-\infty}^{+\infty} M\left(|\omega|+\left|y_{*}\right|\right)^{s} e^{-\nu_{2} \omega^{2} \gamma t} d \omega \\
& \leq \exp \left[-y_{*}^{2} \nu_{2} \gamma t\right] \int_{-\infty}^{+\infty} M 2^{s-1}\left(|\omega|^{s}+\left|y_{*}\right|^{s}\right) e^{-\nu_{2} \omega^{2} \gamma t} d \omega
\end{aligned}
$$

The desired expressions (5.174) for $0 \leq \rho \leq \delta_{\omega}$ now follow from the identities

$$
\begin{aligned}
\int_{-\infty}^{+\infty} e^{-\nu_{2} \omega^{2} \gamma t} d \omega & =\sqrt{\frac{\pi}{\nu_{2} \gamma t}}, \\
\int_{-\infty}^{+\infty} \omega^{2} e^{-\nu_{2} \omega^{2} \gamma t} d \omega & =\frac{1}{2}\left(\nu_{2} \gamma t\right)^{-1} \sqrt{\frac{\pi}{\nu_{2} \gamma t}} \\
\int_{-\infty}^{+\infty} \omega^{4} e^{-\nu_{2} \omega^{2} \gamma t} d \omega & =\frac{3}{4}\left(\nu_{2} \gamma t\right)^{-2} \sqrt{\frac{\pi}{\nu_{2} \gamma t}} .
\end{aligned}
$$

On the other hand, for $\rho \geq \delta_{\omega}$, we compute

$$
\begin{aligned}
\left|\mathcal{W}_{l ; \gamma ; \Gamma_{3}}^{(\wp)}(t)\right| & \leq(\gamma t)^{1 / 2} \exp \left[-\delta_{\omega}^{2} \nu_{2} \gamma t\right] \int_{-\delta_{\omega}}^{\delta_{\omega}} M\left(\left|2 \delta_{\omega}\right|^{s}\right) e^{-\nu_{2} \omega^{2} \gamma t} d \omega \\
& \leq(\gamma t)^{1 / 2} \exp \left[-\delta_{\omega}^{2} \nu_{2} \gamma t\right]\left(2 \delta_{\omega} M\right) \\
& \leq M(\gamma t)^{1 / 2} \exp \left[-\delta_{\omega}^{2} \nu_{2} \gamma t\right]
\end{aligned}
$$

which suffices to establish (5.175). 
We remark that the bounds (5.168) and (5.174) all involve the quantity $v_{l ; \gamma}(t)$. We augment these results with the following uniform estimates.

Lemma 5.10. For all $\gamma \geq 1, t \geq 1$ and $l \in \mathbb{Z}$, we have the uniform bounds

$$
\begin{aligned}
&\left|v_{\gamma ; l}(t)\right| \leq \sqrt{\frac{\pi}{\nu_{2}}}, \\
&\left|\rho v_{\gamma ; l}(t)\right| \leq \sqrt{\frac{\pi}{\nu_{2}}} e^{-1 / 2} \sqrt{\frac{1}{2 \nu_{2} \gamma t}}, \\
&\left|\rho^{2} v_{\gamma ; l}(t)\right| \leq \sqrt{\frac{\pi}{\nu_{2}}} e^{-1} \frac{1}{\nu_{2} \gamma t}, \\
&\left|\rho^{3} v_{\gamma ; l}(t)\right| \leq \sqrt{\frac{\pi}{\nu_{2}}} e^{-3 / 2}\left(\frac{3}{2 \nu_{2} \gamma t}\right)^{3 / 2},
\end{aligned}
$$

again with $\rho=\rho(l, t ; \gamma)$.

Proof. Observe first that for all $x \in \mathbb{R}$ and $\alpha>0$ we have the bounds

$$
|x| \exp \left[-\alpha x^{2}\right] \leq e^{-1 / 2} \sqrt{\frac{1}{2 \alpha}}, \quad x^{2} \exp \left[-\alpha x^{2}\right] \leq \alpha^{-1} e^{-1}, \quad\left|x^{3}\right| \exp \left[-\alpha x^{2}\right] \leq\left(\frac{3}{2 \alpha}\right)^{3 / 2} e^{-3 / 2} .
$$

Using the expression

$$
v_{\gamma ; l}(t)=\sqrt{\frac{\pi}{\nu_{2}}} \exp \left[-\nu_{2} \rho^{2} \gamma t\right]
$$

the desired estimates (5.192) now follow immediately.

Taking a time derivative in (5.165) yields the expression

$$
\dot{v}_{l ; \gamma}(t)=(\gamma t)^{1 / 2} \int_{-\infty}^{\infty}\left[\frac{1}{2} t^{-1}+i \omega \nu_{1}-\gamma \nu_{2} \omega^{2}\right] \exp \left[i \omega\left(l+\nu_{1} t\right)\right] \exp \left[-\nu_{2} \omega^{2} \gamma t\right] d \omega
$$

which in view of the identities (5.170) can be written as

$$
\begin{aligned}
\dot{v}_{\gamma ; l}(t) & =\left[\frac{1}{2} t^{-1}-\nu_{1} \rho(l, t ; \gamma)+\gamma \nu_{2} \rho^{2}(l, t ; \gamma)-\frac{1}{2} \gamma(\gamma t)^{-1}\right] v_{l ; \gamma}(t) \\
& =\left[-\nu_{1} \rho+\gamma \nu_{2} \rho^{2}\right] v_{l ; \gamma}(t),
\end{aligned}
$$

again with $\rho=\rho(l, t ; \gamma)$. For convenience, we note that Lemma 5.10 implies the uniform bound

$$
\left|\dot{v}_{l ; \gamma}(t)\right| \leq \nu_{1} \sqrt{\frac{\pi}{\nu_{2}}} e^{-1 / 2} \sqrt{\frac{1}{2 \nu_{2} \gamma t}}+\frac{1}{t} e^{-1} \sqrt{\frac{\pi}{\nu_{2}}}
$$

for all $\gamma \geq 1, t \geq 1$ and $l \in \mathbb{Z}$.

For any sequence $v \in \ell^{\infty}(\mathbb{Z} ; \mathbb{R})$, let us define the quantity

$$
[\mathcal{K} v]_{l}:=\alpha_{\mu}^{\diamond} \pi_{l ; \mu}^{\diamond} v+\alpha_{\mu \mu^{\prime}}^{\diamond} \pi_{l ; \mu \mu^{\prime}}^{\diamond} v .
$$

A short computation using Lemma 5.5 shows that for some $\kappa_{3} \in \mathbb{R}$, we have

$$
\begin{aligned}
{\left[\mathcal{K} v_{; \gamma}(t)\right]_{l}=(\gamma t)^{1 / 2} \int_{-\infty}^{\infty}\left[\alpha_{\mu}^{\diamond}\left(e^{i \sigma_{\mu} \omega}-1\right)+\alpha_{\mu \mu^{\prime}}^{\diamond \diamond}\left(e^{i \sigma_{\mu} \omega}-1\right)\left(e^{i \sigma_{\mu^{\prime}} \omega}-1\right)\right] } & \quad \times \exp \left[i \omega\left(l+\nu_{1} t\right)\right] \exp \left[-\nu_{2} \omega^{2} \gamma t\right] d \omega \\
= & (\gamma t)^{1 / 2} \int_{-\infty}^{\infty}\left[i \nu_{1} \omega-\nu_{2} \omega^{2}+i \kappa_{3} \omega^{3}+\mathcal{O}_{4}(\omega)\right] \exp \left[i \omega\left(l+\nu_{1} t\right)\right] \exp \left[-\nu_{2} \omega^{2} \gamma t\right] d \omega .
\end{aligned}
$$

Here for any $s \in\{0,2,4\}$, we have introduced the notation $\mathcal{O}_{s}(\omega)$ to denote a function that satisfies the conditions (5.171) - (5.172) for some $M>1$. 
An important role in the sequel will be reserved for the quantity

$$
\mathcal{S}_{l ; \gamma}(t)=\dot{v}_{l ; \gamma}(t)-\frac{1}{2}(\gamma t)^{-1} v_{l ; \gamma}(t)-\left[\mathcal{K} v_{; \gamma}(t)\right]_{l},
$$

for which we can compute

$$
\begin{gathered}
\mathcal{S}_{l ; \gamma}(t)=(\gamma t)^{1 / 2} \int_{-\infty}^{\infty}\left[\frac{1}{2} t^{-1}+i \omega \nu_{1}-\gamma \nu_{2} \omega^{2}-\frac{1}{2}(\gamma t)^{-1}-i \nu_{1} \omega+\nu_{2} \omega^{2}-i \kappa_{3} \omega^{3}-\mathcal{O}_{4}(\omega)\right] \\
\quad \times \exp \left[i \omega\left(l+\nu_{1} t\right)\right] \exp \left[-\nu_{2} \omega^{2} \gamma t\right] d \omega \\
=(\gamma t)^{1 / 2} \int_{-\infty}^{\infty}\left[\frac{1}{2}(\gamma-1)(\gamma t)^{-1}-(\gamma-1) \nu_{2} \omega^{2}-i \kappa_{3} \omega^{3}-\mathcal{O}_{4}(\omega)\right] \\
\times \exp \left[i \omega\left(l+\nu_{1} t\right)\right] \exp \left[-\nu_{2} \omega^{2} \gamma t\right] d \omega .
\end{gathered}
$$

We conclude this subsection by obtaining near field $\left(|\rho| \leq \delta_{\omega}\right)$ and far field $\left(|\rho| \geq \delta_{\omega}\right)$ bounds on the various quantities introduced here, which will allow us to obtain useful bounds on the numbered expressions in (5.137).

Lemma 5.11. Pick $\delta_{\omega}>0$ sufficiently small. There exists a constant $C_{2}=C_{2}\left(\delta_{\omega}\right)>1$ such that for all $\gamma \geq 1, t \geq 1$ and all $l \in \mathbb{Z}$ for which $|\rho(l, t ; \gamma)| \leq \delta_{\omega}$, we have the bound

$$
\begin{gathered}
\left|\mathcal{S}_{l ; \gamma}(t)-(\gamma-1) \nu_{2} \rho^{2} v_{l ; \gamma}(t)\right| \leq C_{2}\left[|\rho|^{3}+|\rho|(\gamma t)^{-1}+(\gamma t)^{-2}\right] v_{l ; \gamma}(t) \\
+C_{2}(\gamma t)^{1 / 2} e^{-\nu_{2} \delta_{\omega}^{2} \gamma t}
\end{gathered}
$$

in which $\rho=\rho(l, \gamma ; t)$.

Proof. This estimate follows directly from (5.201), using the identities (5.170) and the bounds (5.174).

Lemma 5.12. Pick $\delta_{\omega}>0$ sufficiently small. There exists a constant $C_{2}=C_{2}\left(\delta_{\omega}\right)>1$ such that for all $\gamma \geq 1, t \geq 1$ and all $l \in \mathbb{Z}$ for which $|\rho(l, t ; \gamma)| \leq \delta_{\omega}$, we have the bounds

$$
\begin{aligned}
& \left|\pi_{l}^{\diamond} v_{; \gamma}(t)\right| \leq C_{2}\left[|\rho|+(\gamma t)^{-1}\right] v_{l ; \gamma}(t)+C_{2}(\gamma t)^{1 / 2} e^{-\nu_{2} \delta_{\omega}^{2} \gamma t} \\
& \left|\pi_{l}^{\diamond \diamond} v_{; \gamma}(t)\right| \leq C_{2}\left[\rho^{2}+(\gamma t)^{-1}\right] v_{l ; \gamma}(t)+C_{2}(\gamma t)^{1 / 2} e^{-\nu_{2} \delta_{\omega}^{2} \gamma t} \\
& \left|\pi_{l}^{\diamond \diamond \diamond} v_{; \gamma}(t)\right| \leq C_{2}\left[|\rho|^{3}+|\rho|(\gamma t)^{-1}+(\gamma t)^{-2}\right] v_{l ; \gamma}(t)+C_{2}(\gamma t)^{1 / 2} e^{-\nu_{2} \delta_{\omega}^{2} \gamma t}
\end{aligned}
$$

in which $\rho=\rho(l, \gamma ; t)$.

Proof. Observing that

$$
\begin{aligned}
\pi_{l ; \mu}^{\diamond} v_{; \gamma}(t) & =(\gamma t)^{1 / 2} \int_{-\infty}^{\infty}\left(e^{i \sigma_{\mu} \omega}-1\right) \exp \left[i \omega\left(l+\nu_{1} t\right)\right] \exp \left[-\nu_{2} \omega^{2} \gamma t\right] d \omega \\
& =(\gamma t)^{1 / 2} \int_{-\infty}^{\infty}\left(i \sigma_{\mu} \omega+\mathcal{O}_{2}(\omega)\right) \exp \left[i \omega\left(l+\nu_{1} t\right)\right] \exp \left[-\nu_{2} \omega^{2} \gamma t\right] d \omega,
\end{aligned}
$$

the identities (5.170) and the bounds (5.174) suffice to obtain the desired estimate on $\pi_{l}^{\diamond} v_{; \gamma}(t)$. The other estimates can be obtained in a similar fashion.

Lemma 5.13. Pick $\delta_{\omega}>0$ sufficiently small. There exists a constant $C_{2}=C_{2}\left(\delta_{\omega}\right)>1$ such that for all $\gamma \geq 1, t \geq 1$ and all $l \in \mathbb{Z}$ for which $|\rho(l, t ; \gamma)| \leq \delta_{\omega}$, we have the bounds

$$
\begin{array}{cc}
\left|\dot{v}_{l ; \gamma}(t)\right| \leq & C_{2}\left[\rho+\gamma \rho^{2}\right] v_{l ; \gamma}(t) \\
\left|\pi_{l}^{\diamond} \dot{j}_{; \gamma}(t)\right| \leq & C_{2}\left[\gamma(\gamma t)^{-1}|\rho|+\rho^{2}+(\gamma t)^{-1}+\gamma|\rho|^{3}\right] v_{l ; \gamma}(t) \\
& +\gamma C_{2}(\gamma t)^{1 / 2} e^{-\nu_{2} \delta_{\omega}^{2} \gamma t}, \\
\left|\pi_{l}^{\diamond \diamond} \dot{v}_{; \gamma}(t)\right| \leq & C_{2}\left[(\gamma t)^{-1} \gamma \rho^{2}+\gamma(\gamma t)^{-2}+|\rho|^{3}+(\gamma t)^{-1}|\rho|+\gamma \rho^{4}\right] v_{l ; \gamma}(t) \\
& +\gamma C_{2}(\gamma t)^{1 / 2} e^{-\nu_{2} \delta_{\omega}^{2} \gamma t},
\end{array}
$$


in which $\rho=\rho(l, \gamma ; t)$.

Proof. For convenience, we assume throughout this proof that $\rho \geq 0$. The bound for $\dot{v}_{l ; \gamma}(t)$ follows directly from (5.196). Moving on to the second estimate, we write

$$
\begin{aligned}
\pi_{l ; \mu}^{\diamond} \dot{v}_{; \gamma}(t)=(\gamma t)^{1 / 2} \int_{-\infty}^{\infty}[ & \left.\left(i \sigma_{\mu} \omega+\mathcal{O}_{2}(\omega)\right)\left(\frac{1}{2} t^{-1}+i \omega \nu_{1}-\gamma \nu_{2} \omega^{2}\right)\right] \\
& \times \exp \left[i \omega\left(l+\nu_{1} t\right)\right] \exp \left[-\nu_{2} \omega^{2} \gamma t\right] d \omega \\
=(\gamma t)^{1 / 2} \int_{-\infty}^{\infty}\left[\frac{1}{2} t^{-1} i \sigma_{\mu} \omega+\frac{1}{2} t^{-1} \mathcal{O}_{2}(\omega)+\mathcal{O}_{2}(\omega)-i \sigma_{\mu} \gamma \nu_{2} \omega^{3}+\gamma \mathcal{O}_{4}(\omega)\right] & \times \exp \left[i \omega\left(l+\nu_{1} t\right)\right] \exp \left[-\nu_{2} \omega^{2} \gamma t\right] d \omega .
\end{aligned}
$$

Using (5.170) and (5.174), we hence see that there exists $C_{2}^{\prime}>1$ for which

$$
\begin{gathered}
\left|\pi_{l ; \mu}^{\diamond} \dot{v}_{; \gamma}(t)\right| \leq C_{2}^{\prime}\left[t^{-1}\left(\rho+\rho^{2}+(\gamma t)^{-1}\right)+\rho^{2}+(\gamma t)^{-1}+\gamma\left(\rho^{3}+\rho(\gamma t)^{-1}+\rho^{4}+(\gamma t)^{-2}\right)\right] \\
+\gamma C_{2}^{\prime}(\gamma t)^{1 / 2} e^{-\nu_{2} \delta_{\omega}^{2} \gamma t}
\end{gathered}
$$

from which the desired estimate immediately follows.

To obtain the third estimate, we note that there exists $\kappa_{3} \in \mathbb{R}$ for which

$$
\begin{gathered}
\pi_{l ; \mu \mu^{\prime}}^{\diamond \diamond} \dot{v}_{; \gamma}(t)=(\gamma t)^{1 / 2} \int_{-\infty}^{\infty}[ \\
\left.\quad\left(-\sigma_{\mu} \sigma_{\mu^{\prime}} \omega^{2}+i \kappa_{3} \omega^{3}+\mathcal{O}_{4}(\omega)\right)\left(\frac{1}{2} t^{-1}+i \omega \nu_{1}-\gamma \nu_{2} \omega^{2}\right)\right] \\
\quad \times \exp \left[i \omega\left(l+\nu_{1} t\right)\right] \exp \left[-\nu_{2} \omega^{2} \gamma t\right] d \omega \\
=(\gamma t)^{1 / 2} \int_{-\infty}^{\infty}\left[\frac{1}{2} t^{-1} \mathcal{O}_{2}(\omega)-i \omega^{3} \nu_{1} \sigma_{\mu} \sigma_{\mu^{\prime}}+\mathcal{O}_{4}(\omega)+\gamma \mathcal{O}_{4}(\omega)\right] \\
\times \exp \left[i \omega\left(l+\nu_{1} t\right)\right] \exp \left[-\nu_{2} \omega^{2} \gamma t\right] d \omega .
\end{gathered}
$$

Using (5.170) and (5.174), we hence see that there exists $C_{2}^{\prime}>1$ for which

$$
\begin{gathered}
\left|\pi_{l ; \mu \mu^{\prime}}^{\diamond} \dot{z}_{; \gamma}(t)\right| \leq C_{2}^{\prime}\left[t^{-1}\left(\rho^{2}+(\gamma t)^{-1}\right)+\rho^{3}+(\gamma t)^{-1} \rho+(\gamma+1)\left(\rho^{4}+(\gamma t)^{-2}\right)\right] \\
+\gamma C_{2}^{\prime}(\gamma t)^{1 / 2} e^{-\nu_{2} \delta_{\omega}^{2} \gamma t}
\end{gathered}
$$

from which the desired estimate immediately follows.

Lemma 5.14. Pick $\delta_{\omega}>0$ sufficiently small. There exists a constant $C_{2}=C_{2}\left(\delta_{\omega}\right)>1$ such that for all $\gamma \geq 1, t \geq 1$ and all $l \in \mathbb{Z}$ for which $|\rho(l, t ; \gamma)| \leq \delta_{\omega}$, we have the bounds

$$
\begin{aligned}
\left|\dot{v}_{; \gamma}(t)-\alpha_{\mu}^{\diamond} \pi_{; \mu}^{\diamond} v_{; \gamma}(t)\right| \leq & C_{2}\left[\rho^{2}+(\gamma t)^{-1}\right] v_{l ; \gamma}(t) \\
& +C_{2}(\gamma t)^{1 / 2} e^{-\nu_{2} \delta_{\omega}^{2} \gamma t} \\
\left|\pi_{l}^{\diamond}\left[\dot{v}_{l ; \gamma}(t)-\alpha_{\mu}^{\diamond} \pi_{l ; \mu}^{\diamond} v_{; \gamma}(t)\right]\right| \leq \quad C_{2}[ & \left.(\gamma t)^{-1} \gamma|\rho|+\gamma(\gamma t)^{-2}+\gamma|\rho|^{3}\right] v_{l ; \gamma}(t) \\
& +\gamma C_{2}(\gamma t)^{1 / 2} e^{-\nu_{2} \delta_{\omega}^{2} \gamma t}
\end{aligned}
$$

in which $\rho=\rho(l, \gamma ; t)$.

Proof. As before, we restrict ourselves to the setting $\rho \geq 0$. First of all, we write

$$
w_{l ; \gamma}(t)=\dot{v}_{l ; \gamma}(t)-\alpha_{\mu}^{\diamond} \pi_{l ; \mu}^{\diamond} v_{; \gamma}(t)
$$


and note that there exist $\kappa_{2}, \kappa_{3} \in \mathbb{R}$ for which we can compute

$$
\begin{gathered}
w_{l ; \gamma}(t)=(\gamma t)^{1 / 2} \int_{-\infty}^{\infty}\left[\frac{1}{2} t^{-1}+i \omega \nu_{1}-\gamma \nu_{2} \omega^{2}-\alpha_{\mu}^{\diamond}\left(e^{i \sigma_{\mu} \omega}-1\right)\right] \\
\times \exp \left[i \omega\left(l+\nu_{1} t\right)\right] \exp \left[-\nu_{2} \omega^{2} \gamma t\right] d \omega \\
=(\gamma t)^{1 / 2} \int_{-\infty}^{\infty}\left[\frac{1}{2} t^{-1}+i \omega \nu_{1}-\gamma \nu_{2} \omega^{2}-i \omega \nu_{1}+\kappa_{2} \omega^{2}+i \kappa_{3} \omega^{3}+\mathcal{O}(\omega)\right] \\
\times \exp \left[i \omega\left(l+\nu_{1} t\right)\right] \exp \left[-\nu_{2} \omega^{2} \gamma t\right] d \omega \\
=(\gamma t)^{1 / 2} \int_{-\infty}^{\infty}\left[\frac{1}{2} t^{-1}-\gamma \nu_{2} \omega^{2}+\kappa_{2} \omega^{2}+i \kappa_{3} \omega^{3}+\mathcal{O}_{4}(\omega)\right] \\
\times \exp \left[i \omega\left(l+\nu_{1} t\right)\right] \exp \left[-\nu_{2} \omega^{2} \gamma t\right] d \omega .
\end{gathered}
$$

In particular, invoking (5.170) and (5.174) and exploiting a partial cancellation in the first two terms, we see that there exists $C_{2}^{\prime}>1$ for which

$$
\begin{gathered}
\left|w_{l ; \gamma}(t)\right| \leq C_{2}^{\prime}\left[\rho^{2}+(\gamma t)^{-1}+\rho^{3}+\rho(\gamma t)^{-1}+\rho^{4}+(\gamma t)^{-2}\right] v_{l ; \gamma}(t) \\
+C_{2}^{\prime}(\gamma t)^{1 / 2} e^{-\nu_{2} \delta_{\omega}^{2} \gamma t}
\end{gathered}
$$

which suffices to obtain the first stated estimate.

Moving on to the second estimate, we compute

$$
\begin{aligned}
& \pi_{l ; \mu}^{\diamond} w_{; \gamma}(t)=(\gamma t)^{1 / 2} \int_{-\infty}^{\infty}[\left.\left(i \sigma_{\mu} \omega+\mathcal{O}_{2}(\omega)\right)\left(\frac{1}{2} t^{-1}-\gamma \nu_{2} \omega^{2}+\kappa_{2} \omega^{2}+i \kappa_{3} \omega^{3}+\mathcal{O}_{4}(\omega)\right)\right] \\
& \times \exp \left[i \omega\left(l+\nu_{1} t\right)\right] \exp \left[-\nu_{2} \omega^{2} \gamma t\right] d \omega \\
&=(\gamma t)^{1 / 2} \int_{-\infty}^{\infty}\left[\frac{1}{2} t^{-1}\left(i \sigma_{\mu} \omega+\mathcal{O}_{2}(\omega)\right)-\gamma i \sigma_{\mu} \nu_{2} \omega^{3}+i \sigma_{\mu} \kappa_{2} \omega^{3}+(1+\gamma) \mathcal{O}_{4}(\omega)\right] \\
& \times \exp \left[i \omega\left(l+\nu_{1} t\right)\right] \exp \left[-\nu_{2} \omega^{2} \gamma t\right] d \omega .
\end{aligned}
$$

As before, there exists $C_{2}^{\prime}>1$ for which

$$
\begin{gathered}
\left|\pi_{l ; \mu}^{\diamond} w_{; \gamma}(t)\right| \leq C_{2}^{\prime}\left[t^{-1}\left(\rho+(\gamma t)^{-1}\right)+(\gamma+1)\left(\rho^{3}+\rho(\gamma t)^{-1}+(\gamma t)^{-2}\right)\right] v_{l ; \gamma}(t) \\
+\gamma C_{2}^{\prime}(\gamma t)^{1 / 2} e^{-\nu_{2} \delta_{\omega}^{2} \gamma t}
\end{gathered}
$$

which suffices to complete the proof.

Lemma 5.15. Pick a sufficiently small $\delta_{\rho}>0$. There exists a constant $C_{3}=C_{3}\left(\delta_{\rho}\right)>1$ so that for every $\gamma \geq 1$, every $t \geq 1$ and every $l \in \mathbb{Z}$ for which $|\rho(l, t ; \gamma)| \geq \delta_{\rho}$, we have the bounds

$$
\begin{aligned}
\left|v_{\gamma ; l}(t)\right|+\left|\pi_{l}^{\diamond} v_{; \gamma}(t)\right|+\left|\pi_{l}^{\diamond \diamond} v_{; \gamma}(t)\right|+\left|\pi_{l}^{\diamond \diamond \diamond} v_{; \gamma}(t)\right| & \leq C_{3}(\gamma t)^{1 / 2} \exp \left[-\nu_{2} \delta_{\rho}^{2} \gamma t\right], \\
\left|\dot{v}_{\gamma ; l}(t)\right|+\left|\pi_{l}^{\diamond} \dot{v}_{; \gamma}(t)\right|+\left|\pi_{l}^{\diamond \diamond} \dot{v}_{; \gamma}(t)\right| & \leq \gamma C_{3}(\gamma t)^{1 / 2} \exp \left[-\nu_{2} \delta_{\rho}^{2} \gamma t\right], \\
\left|\mathcal{S}_{l ; \gamma}(t)\right| & \leq \gamma C_{3}(\gamma t)^{1 / 2} \exp \left[-\nu_{2} \delta_{\rho}^{2} \gamma t\right] .
\end{aligned}
$$

Proof. These estimates follow directly from (5.175) together with the identities (5.196) and (5.201).

\subsection{The function $\theta$}

In this subsection we scale the plateau function $v_{; \gamma}$ defined in (5.165) by a global factor that decays very slowly in time. The resulting function $\theta$ controls the phase-shifts of the sub-solution $u^{-}$in the direction transverse to the wave propagation.

Throughout this subsection, we write

$$
\alpha=\alpha(\gamma)=\frac{1}{4 \gamma} .
$$


For every $\beta \geq 1$ and $\gamma \geq 1$, we now define the $C^{1}$-smooth function

$$
\theta_{; \beta, \gamma}:[1, \infty) \rightarrow \ell^{\infty}(\mathbb{Z} ; \mathbb{R})
$$

that acts as

$$
\theta_{l ; \beta, \gamma}(t)=\beta t^{-\alpha} v_{l ; \gamma}(t)
$$

and can be differentiated as

$$
\dot{\theta}_{l ; \beta, \gamma}(t)=\beta t^{-\alpha}\left[-\frac{1}{4}(\gamma t)^{-1} v_{l ; \gamma}(t)+\dot{v}_{l ; \gamma}(t)\right] .
$$

An important role in the sequel will be played by the quantities

$$
\mathcal{T}_{l ; \beta, \gamma}(t)=\dot{\theta}_{l ; \beta, \gamma}(t)-\alpha_{\mu}^{\diamond} \pi_{l ; \mu}^{\diamond} \theta_{; \beta, \gamma}(t)-\alpha_{\mu \mu^{\prime}}^{\diamond \diamond} \pi_{l ; \mu \mu^{\prime}}^{\diamond} \theta_{; \beta, \gamma}(t) .
$$

Recalling the linear operator $\mathcal{K}$ defined in (5.198), a short computation shows that

$$
\begin{aligned}
\mathcal{T}_{l ; \beta, \gamma}(t) & =\beta t^{-\alpha}\left[\dot{v}_{l ; \gamma}(t)-\frac{1}{4}(\gamma t)^{-1} v_{l ; \gamma}-\left[\mathcal{K} v_{; \gamma}(t)\right]_{l}\right] \\
& =\beta t^{-\alpha}\left[\frac{1}{4}(\gamma t)^{-1} v_{l ; \gamma}(t)+\mathcal{S}_{l ; \gamma}(t)\right] .
\end{aligned}
$$

In order to obtain a useful bound on $\mathcal{T}_{l ; \beta, \gamma}(t)$, we introduce the strictly positive expressions

$$
\mathcal{Q}_{l ; \beta, \gamma}(t)=\frac{1}{8} \beta t^{-\alpha}\left[\nu_{2} \gamma \rho^{2}+(\gamma t)^{-1}\right] v_{l ; \gamma}(t),
$$

with $\rho=\rho(l, t ; \gamma)$.

Lemma 5.16. Pick a sufficiently small $\delta_{\omega}>0$. There exists constants $C_{\mathcal{T}}=C_{\mathcal{T}}\left(\delta_{\omega}\right)>0$ and $\gamma_{*}=\gamma_{*}\left(\delta_{\omega}\right) \geq 1$ such that for all $\gamma \geq \gamma_{*}$, all $\beta \geq 1$, all $t \geq 1$ and all $l \in \mathbb{Z}$ for which $|\rho(l, t ; \gamma)| \leq \delta_{\omega}$, we have

$$
\mathcal{T}_{l ; \beta, \gamma}(t) \geq \mathcal{Q}_{l ; \beta, \gamma}(t)-\beta C_{\mathcal{T}}(\gamma t)^{1 / 2} e^{-\nu_{2} \delta_{\omega}^{2} \gamma t} .
$$

Proof. Notice first that $\gamma_{*} \geq 2$ ensures $\gamma_{*}-1 \geq \frac{1}{2} \gamma_{*}$. In addition, using the elementary estimate

$$
\begin{aligned}
\rho^{3}+\rho(\gamma t)^{-1}+(\gamma t)^{-2} & \leq \rho^{3}+\rho^{2}+2(\gamma t)^{-2} \\
& =\nu_{2} \gamma \rho^{2}\left[\nu_{2}^{-1} \gamma^{-1} \rho+\nu_{2}^{-1} \gamma^{-1}\right]+(\gamma t)^{-1}\left(2 \frac{1}{\gamma t}\right),
\end{aligned}
$$

Lemma 5.11 implies that it suffices to pick $\gamma_{*} \geq 2$ in such a way that

$$
C_{2}\left[\nu_{2}^{-1} \gamma_{*}^{-1} \delta_{\omega}+\nu_{2}^{-1} \gamma_{*}^{-1}\right] \leq \frac{1}{8}, \quad 2 C_{2} \gamma_{*}^{-1} \leq \frac{1}{8} .
$$

In the remainder of this subsection we obtain near field $\left(|\rho| \leq \delta_{\omega}\right)$, far field $\left(|\rho| \geq \delta_{\omega}\right)$ and global bounds on various terms involving $\theta_{; \beta, \gamma}(t)$, which will allow us to obtain useful bounds on the numbered expressions in (5.137).

Lemma 5.17. Pick $\delta_{\omega}>0$ sufficiently small. There exists a constant $C_{4}=C_{4}\left(\delta_{\omega}\right)>1$ such that for all $\beta \geq 1$, all $\gamma \geq 1$, all $t \geq 1$ and all $l \in \mathbb{Z}$ for which $|\rho(l, t ; \gamma)| \leq \delta_{\omega}$, we have the bounds

$$
\begin{array}{cc}
\left|\pi_{l}^{\diamond} \theta_{; \beta, \gamma}(t)\right| \leq & \beta C_{4} t^{-\alpha}\left[|\rho|+(\gamma t)^{-1}\right] v_{l ; \gamma}(t) \\
& +\beta C_{4}(\gamma t)^{1 / 2} e^{-\nu_{2} \delta_{\omega}^{2} \gamma t} \\
\left|\pi_{l}^{\diamond \diamond} \theta_{; \beta, \gamma}(t)\right| \leq & \beta C_{4} t^{-\alpha}\left[\rho^{2}+(\gamma t)^{-1}\right] v_{l ; \gamma}(t) \\
& +\beta C_{4}(\gamma t)^{1 / 2} e^{-\nu_{2} \delta_{\omega}^{2} \gamma t} \\
\left|\pi_{l}^{\diamond \diamond \diamond} \theta_{; \beta, \gamma}(t)\right| \leq & \beta C_{4} t^{-\alpha}\left[|\rho|^{3}+|\rho|(\gamma t)^{-1}+(\gamma t)^{-2}\right] v_{l ; \gamma}(t) \\
& +\beta C_{4}(\gamma t)^{1 / 2} e^{-\nu_{2} \delta_{\omega}^{2} \gamma t}
\end{array}
$$

with $\rho=\rho(l, t ; \gamma)$. 
Proof. In view of the identity (5.219), these bounds follow immediately from Lemma 5.12.

Lemma 5.18. Pick $\delta_{\omega}>0$ sufficiently small. There exists a constant $C_{4}=C_{4}\left(\delta_{\omega}\right)>1$ such that for all $\beta \geq 1$, all $\gamma \geq 1$, all $t \geq 1$ and all $l \in \mathbb{Z}$ for which $|\rho(l, t ; \gamma)| \leq \delta_{\omega}$, we have the bounds

$$
\begin{aligned}
& \left|\dot{\theta}_{l ; \beta, \gamma}(t)\right| \leq \beta C_{4} t^{-\alpha}\left[\rho+\gamma \rho^{2}+(\gamma t)^{-1}\right] v_{l ; \gamma}(t), \\
& \left|\pi_{l}^{\diamond} \dot{\theta}_{; \beta, \gamma}(t)\right| \leq \beta C_{4} t^{-\alpha}\left[\gamma(\gamma t)^{-1}|\rho|+\rho^{2}+(\gamma t)^{-1}+\gamma|\rho|^{3}\right] v_{l ; \gamma}(t) \\
& +\beta \gamma C_{4}(\gamma t)^{1 / 2} e^{-\nu_{2} \delta_{\omega}^{2} \gamma t}, \\
& \left|\pi_{l}^{\diamond \diamond} \dot{\theta}_{; \beta, \gamma}(t)\right| \leq \beta C_{4} t^{-\alpha}\left[(\gamma t)^{-1} \gamma \rho^{2}+\gamma(\gamma t)^{-2}+|\rho|^{3}+(\gamma t)^{-1}|\rho|+\gamma \rho^{4}\right] v_{l ; \gamma}(t) \\
& +\beta \gamma C_{4}(\gamma t)^{1 / 2} e^{-\nu_{2} \delta_{\omega}^{2} \gamma t},
\end{aligned}
$$

with $\rho=\rho(l, t ; \gamma)$.

Proof. Combining the results from Lemmas 5.12 and 5.13, these estimates follow directly from (5.220).

Lemma 5.19. Pick $\delta_{\omega}>0$ sufficiently small. There exists a constant $C_{4}=C_{4}\left(\delta_{\omega}\right)>1$ such that for all $\beta \geq 1$, all $\gamma \geq 1$, all $t \geq 1$ and all $l \in \mathbb{Z}$ for which $|\rho(l, t ; \gamma)| \leq \delta_{\omega}$, we have the bounds

$$
\begin{array}{cc}
\left|\dot{\theta}_{l ; \beta, \gamma}(t)-\alpha_{\mu}^{\diamond} \pi_{; \mu}^{\diamond} \theta_{; \beta, \gamma}(t)\right| \leq & \beta C_{4} t^{-\alpha}\left[\rho^{2}+(\gamma t)^{-1}\right] v_{l ; \gamma}(t) \\
& +\beta C_{4}(\gamma t)^{1 / 2} e^{-\nu_{2} \delta_{\omega}^{2} \gamma t}, \\
\mid \pi_{l}^{\diamond}\left[\dot{\theta}_{; \beta, \gamma, t_{*}}(t)-\alpha_{\mu}^{\diamond} \pi_{; \mu}^{\diamond} \theta_{; \beta, \gamma}(t)\right] \leq \quad \beta C_{4} t^{-\alpha}\left[(\gamma t)^{-1} \gamma|\rho|+\gamma(\gamma t)^{-2}+\gamma|\rho|^{3}\right] v_{l ; \gamma}(t) \\
+\beta \gamma C_{4}(\gamma t)^{1 / 2} e^{-\nu_{2} \delta_{\omega}^{2} \gamma t}
\end{array}
$$

with $\rho=\rho(l, t ; \gamma)$.

Proof. Combining the results from Lemmas 5.12 and 5.14, these estimates follow directly from (5.220).

Lemma 5.20. Pick a sufficiently small $\delta_{\rho}>0$. There exists a constant $C_{5}=C_{5}\left(\delta_{\rho}\right)>1$ so that for all $\beta \geq 1$, all $\gamma \geq 1$, all $t \geq 1$ and all $l \in \mathbb{Z}$ for which $|\rho(l, t ; \gamma)| \geq \delta_{\rho}$, we have the bounds

$$
\begin{aligned}
\left|\theta_{l ; \beta, \gamma}(t)\right|+\left|\pi_{l}^{\diamond} \theta_{; \beta, \gamma}(t)\right|+\left|\pi_{l}^{\diamond} \theta_{; \beta, \gamma}(t)\right|+\left|\pi_{l}^{\diamond \diamond \diamond} \theta_{; \beta, \gamma}(t)\right| & \leq \beta C_{5} t^{-\alpha}(\gamma t)^{1 / 2} \exp \left[-\nu_{2} \delta_{\rho}^{2} \gamma t\right], \\
\left|\dot{\theta}_{\gamma ; l}(t)\right|+\left|\pi_{l}^{\diamond} \dot{\theta}_{; \beta, \gamma}(t)\right|+\left|\pi_{l}^{\diamond \diamond} \dot{\theta}_{; \beta, \gamma}(t)\right| & \leq \beta \gamma C_{5} t^{-\alpha}(\gamma t)^{1 / 2} \exp \left[-\nu_{2} \delta_{\rho}^{2} \gamma t\right], \\
\left|\mathcal{T}_{l ; \beta, \gamma}(t)\right| & \leq \beta \gamma C_{5} t^{-\alpha}(\gamma t)^{1 / 2} \exp \left[-\nu_{2} \delta_{\rho}^{2} \gamma t\right] .
\end{aligned}
$$

Proof. Using Lemma 5.15, these estimates follow directly from (5.219) and (5.220).

Lemma 5.21. There exist constants $C_{6}>1$ and $\gamma_{*} \geq 1$ such that for all $\gamma \geq \gamma_{*}$, all $\beta \geq 1$, all $t \geq 1$ and all $l \in \mathbb{Z}$, we have the bounds

$$
\begin{aligned}
& \left|\pi_{l}^{\diamond} \theta_{; \beta, \gamma}(t)\right| \leq \beta C_{6} t^{-\alpha}(\gamma t)^{-1 / 2}, \\
& \left|\pi_{l}^{\diamond \diamond} \theta_{; \beta, \gamma}(t)\right| \leq \beta C_{6} t^{-\alpha}(\gamma t)^{-1}, \\
& \left|\pi_{l}^{\diamond \diamond \diamond} \theta_{; \beta, \gamma}(t)\right| \leq \beta C_{6} t^{-\alpha}(\gamma t)^{-3 / 2} .
\end{aligned}
$$


Proof. Note first that for every fixed $\delta_{\omega}>0$, we can obtain

$$
\gamma(\gamma t)^{1 / 2} e^{-\nu_{2} \delta_{\omega}^{2} \gamma t} \leq(\gamma t)^{-3 / 2}
$$

for all $t \geq 1$ and all $\gamma \geq \gamma_{*}$ by picking $\gamma_{*}$ to be sufficiently large. The desired bounds now follow from Lemma 5.17 and Lemma 5.20, upon using the global estimates obtained in Lemma 5.10.

Lemma 5.22. There exist constants $C_{6}>1$ and $\gamma_{*} \geq 1$ such that for all $\gamma \geq \gamma_{*}$, all $\beta \geq 1$, all $t \geq 1$ and all $l \in \mathbb{Z}$, we have the bounds

$$
\begin{aligned}
& \left|\dot{\theta}_{l ; \beta, \gamma}(t)\right| \leq \beta C_{6} t^{-\alpha}\left[(\gamma t)^{-1 / 2}+\gamma(\gamma t)^{-1}\right] \\
& \left|\pi_{l}^{\diamond} \dot{\theta}_{; \beta, \gamma}(t)\right| \leq \beta C_{6} t^{-\alpha}\left[\gamma(\gamma t)^{-3 / 2}+(\gamma t)^{-1}\right], \\
& \left|\pi_{l}^{\diamond \diamond} \dot{\theta}_{; \beta, \gamma *}(t)\right| \leq \beta C_{6} t^{-\alpha}\left[\gamma(\gamma t)^{-2}+(\gamma t)^{-3 / 2}\right] .
\end{aligned}
$$

Proof. Arguing similarly as in the proof of Lemma 5.21, these estimates follow from Lemmas 5.18 and 5.20.

Lemma 5.23. There exist constants $C_{6}>1$ and $\gamma_{*} \geq 1$ such that for all $\gamma \geq \gamma_{*}$, all $\beta \geq 1$, all $t \geq 1$ and all $l \in \mathbb{Z}$, we have the bounds

$$
\left\{\begin{array}{l}
\left|\dot{\theta}_{l ; \beta, \gamma}(t)-\alpha_{\mu}^{\diamond} \pi_{; \mu}^{\diamond} \theta_{; \beta, \gamma}(t)\right| \leq \beta C_{6} t^{-\alpha}(\gamma t)^{-1} \\
\left|\pi_{l}^{\diamond}\left[\dot{\theta}_{; \beta, \gamma}(t)-\alpha_{\mu}^{\diamond} \pi_{; \mu}^{\diamond} \theta_{; \beta, \gamma}(t)\right]\right| \leq \beta C_{6} t^{-\alpha} \gamma(\gamma t)^{-3 / 2}
\end{array}\right.
$$

Proof. Arguing similarly as in the proof of Lemma 5.21, these estimates follow from Lemmas 5.19 and 5.20.

\subsection{Construction of sub-solution}

In this subsection we finally provide the proof of Proposition 5.1. In fact, we set out prove the following result, which is more closely related to the notation we have developed in this section.

Proposition 5.24. Consider any angle $\zeta_{*}$ with $\tan \zeta_{*} \in \mathbb{Q}$ and suppose that $(H g)$ and $(H S)_{\zeta_{*}}$ both hold. Pick $\left(\sigma_{h}, \sigma_{v}\right) \in \mathbb{Z}^{2} \backslash\{(0,0)\}$ with the property that

$$
\sqrt{\sigma_{h}^{2}+\sigma_{v}^{2}}\left(\cos \zeta_{*}, \sin \zeta_{*}\right)=\left(\sigma_{h}, \sigma_{v}\right), \quad \operatorname{gcd}\left(\sigma_{h}, \sigma_{v}\right)=1,
$$

suppose that $(h \Phi)_{\S 3}$ holds for this pair $\left(\sigma_{h}, \sigma_{v}\right)$ with $c>0$ and recall the setting of Lemma 5.3.

Then there exist constants $\delta_{z}>0, \eta_{z}>0, K_{Z}>0, \eta_{\mathcal{N}}>0$ and $K_{\mathcal{N}}>0$ so that the following holds true. Pick any $\epsilon_{2}>0$, any $\beta>1$, any $\Omega_{\perp}>0$ and any $C^{1}$-smooth function $z:[1, \infty) \rightarrow\left(0, \delta_{z}\right]$ that has

$$
\dot{z}(t) \geq-\eta_{z} z(t)
$$

for all $t \geq 1$, together with

$$
\epsilon_{3}:=\inf _{t \geq 1} t^{3 / 2} z(t)>0 .
$$

Then there exists $\gamma=\gamma\left(\epsilon_{2}, \epsilon_{3}, \beta, \Omega_{\perp}\right) \geq 1$ such that the function $u^{-}:[1, \infty) \rightarrow \ell^{\infty}\left(\mathbb{Z}^{2} ; \mathbb{R}\right)$ defined by

$$
u^{-}(t)=\mathrm{J}(\Phi ; t)+\mathrm{J}\left(\pi_{; \mu}^{\diamond} \theta, p_{\mu}^{\diamond} ; t\right)+\mathrm{J}\left(\pi_{\mu \mu^{\prime}}^{\diamond \diamond} \theta, p_{\mu \mu^{\prime}}^{\diamond \diamond} ; t\right)+\mathrm{J}\left(\pi_{\mu}^{\diamond} \theta, \pi_{\mu^{\prime}}^{\diamond} \theta, q_{\mu \mu^{\prime}}^{\diamond} ; t\right)-z(t)
$$

with

$$
\begin{aligned}
& Z(t)=K_{Z} \int_{1}^{t} z(s) d s, \\
& \xi_{n l}(t)=n+c t-\theta_{l ; \beta, \gamma}(t)-Z(t)
\end{aligned}
$$


satisfies the differential inequality

$$
\mathcal{J}_{n l}^{-}(t) \leq-\frac{1}{2} \eta_{z} z(t), \quad(n, l) \in \mathbb{Z}^{2}, \quad t \geq 1
$$

and admits the bound

$$
\left|u^{-}(t)-\mathrm{J}(\Phi ; t)+z(t)\right| \leq \epsilon_{2} t^{-1 / 2}, \quad t \geq 1 .
$$

In addition, for every $(n, l) \in \mathbb{Z}^{2}$ and $t \geq 1$ we have the inequality

$$
\left|\theta_{l+1 ; \beta, \gamma}(t)-\theta_{l ; \beta, \gamma}(t)\right| \leq 1,
$$

which for $|l| \leq \Omega_{\perp}$ can be augmented by

$$
\dot{\xi}_{n l}(t) \geq \frac{c}{2} .
$$

Finally, for any $\nu \in\{1, \ldots, 4\}$, we have the bound

$$
\left|\left[\pi_{n l ; \nu}^{\times}-\pi_{n l ; 5}^{\times}\right] u^{-}(t)\right| \leq K_{\mathcal{N}} e^{-\eta_{\mathcal{N}}\left|\xi_{n l}(t)\right|}, \quad(n, l) \in \mathbb{Z}^{2}, \quad t \geq 1 .
$$

We start our analysis by looking at the auxilliary estimates (5.241), (5.242), (5.243) and (5.244). First of all, Lemma 5.4 implies that there exists $C_{1}^{\prime}>1$ for which

$$
\begin{aligned}
\left|u_{n l}^{-}(t)+z(t)-\mathrm{J}_{n l}(\Phi ; t)\right| & \leq\left|\mathrm{J}_{n l}\left(\pi_{; \mu}^{\diamond} \theta, p_{\mu}^{\diamond} ; t\right)\right|+\left|\mathrm{J}_{n l}\left(\pi_{; \mu \mu^{\prime}}^{\diamond} \theta, p_{\mu \mu^{\prime}}^{\diamond} ; t\right)\right|+\left|\mathrm{J}_{n l}\left(\pi_{\mu}^{\diamond} \theta, \pi_{\mu^{\prime}}^{\diamond} \theta, q_{\mu \mu^{\prime}}^{\diamond} ; t\right)\right| \\
& \leq C_{1}^{\prime}\left[\left|\pi_{l}^{\diamond} \theta(t)\right|+\left|\pi_{l}^{\diamond} \theta(t)\right|+\left|\pi_{l}^{\diamond} \theta(t)\right|^{2}\right] .
\end{aligned}
$$

In addition, the fact that $\operatorname{gcd}\left(\sigma_{h}, \sigma_{v}\right)=1$ implies that $\kappa_{h} \sigma_{h}+\kappa_{v} \sigma_{v}=1$ for some pair $\left(\kappa_{h}, \kappa_{v}\right) \in \mathbb{Z}^{2}$. This means that

$$
\left|\theta_{l+1 ; \beta, \gamma}(t)-\theta_{l ; \beta, \gamma}(t)\right| \leq\left[\left|\kappa_{h}\right|+\left|\kappa_{v}\right|\right]\left\|\pi^{\diamond} \theta_{; \beta, \gamma}(t)\right\|_{\ell^{\infty}\left(\mathbb{Z} ; \mathbb{R}^{5}\right)} .
$$

In particular, the following result suffices to obtain (5.241) and (5.242).

Lemma 5.25. Pick any $\kappa>0$. Then for any $\beta>1$, there exists $\gamma_{*}=\gamma_{*}(\kappa, \beta) \geq 1$, such that for any $\gamma \geq \gamma_{*}$, any $t \geq 1$ and any $l \in \mathbb{Z}$ we have the bounds

$$
\left|\pi_{l}^{\diamond} \theta_{; \beta, \gamma}(t)\right|+\left|\pi_{l}^{\diamond \diamond} \theta_{; \beta, \gamma}(t)\right| \leq \kappa t^{-1 / 2} .
$$

Proof. This follows directly from the global bounds in Lemma 5.21, choosing $\gamma_{*} \gg \beta^{2}$.

In view of the identity

$$
\dot{\xi}_{n l}(t)=c-\dot{\theta}_{l ; \beta, \gamma}(t)-K_{Z} z(t),
$$

the following result can be used to establish (5.243) provided that $|z(t)| \leq \frac{c}{4 K_{z}}$ for all $t \geq 1$.

Lemma 5.26. For any $\beta>1$ and $\Omega_{\perp}>0$, there exist $\gamma_{*}=\gamma_{*}\left(\beta, \Omega_{\perp}\right) \geq 1$, such that for any $\gamma \geq \gamma_{*}$, any $t \geq 1$ and any $l \in \mathbb{Z}$ for which $|l| \leq \Omega_{\perp}$, we have the bound

$$
\left|\dot{\theta}_{l ; \beta, \gamma}\right|<\frac{c}{4} .
$$

Proof. The uniform bound for $\dot{\theta}$ obtained in Lemma 5.22 implies that there exists $t_{0} \geq 1$ such that for every $\gamma \geq 1, t \geq t_{0}$ and $l \in \mathbb{Z}$ we have the bound (5.249).

It hence remains to consider the regime $1 \leq t \leq t_{0}$ and $|l| \leq \Omega_{\perp}$, for which we may estimate

$$
|\rho(l, t ; \gamma)|=\frac{1}{2}\left|\frac{l+\nu_{1} t}{\nu_{2} \gamma t}\right| \leq \frac{1}{2}\left(\nu_{2} \gamma\right)^{-1}\left[\Omega_{\perp}+\left|\nu_{1}\right| t_{0}\right] \leq C_{2}^{\prime} \gamma^{-1}
$$


for some $C_{2}^{\prime}>1$. Using (5.220), we write

$$
\dot{\theta}_{l ; \beta, \gamma}(t)=\beta t^{-\alpha}\left[-\frac{1}{4}(\gamma t)^{-1}-\nu_{1} \rho+\gamma \nu_{2} \rho^{2}\right] v_{l ; \gamma}(t),
$$

which shows that

$$
\left|\dot{\theta}_{l ; \beta, \gamma}(t)\right| \leq \beta\left[\frac{1}{4} \gamma^{-1}+\left|\nu_{1}\right| C_{2}^{\prime} \gamma^{-1}+\gamma \nu_{2}\left(C_{2}^{\prime}\right)^{2} \gamma^{-2}\right]
$$

whenever $1 \leq t \leq t_{0}$ and $|l| \leq \Omega_{\perp}$. Picking $\gamma_{*} \geq 1$ sufficiently large hence establishes the desired bound (5.249).

We note that the final auxilliary estimate $(5.244)$ can be obtained by noting that the bound for the nonlinear expressions $\mathcal{R}_{\mathcal{N} ; \nu}$ obtained in Lemma 5.7 merely requires an a-priori estimate on $\pi^{\diamond} \theta_{; \beta, \gamma}(t)$. Such an estimate can easily be obtained using Lemma 5.25 to restrict $\gamma \geq \gamma_{*} \geq 1$.

It now remains to establish the differential inequality (5.240). To this end, we introduce the expressions

$$
\begin{aligned}
\Theta_{n l ; \beta, \gamma}(t) & =\mathrm{J}_{n l}\left(\dot{\theta}_{; \beta, \gamma}, \Phi^{\prime} ; t\right)-\mathrm{J} n l\left(\alpha_{\mu}^{\diamond} \pi_{\mu}^{\diamond} \theta_{; \beta, \gamma}, \Phi^{\prime} ; t\right)-\mathrm{J} n l\left(\alpha_{\mu}^{\diamond \diamond} \pi_{\mu \mu^{\prime}}^{\diamond} \theta_{; \beta, \gamma}, \Phi^{\prime} ; t\right) \\
& =\mathcal{T}_{l ; \beta, \gamma}(t) \Phi^{\prime}\left(\xi_{n l}(t)\right), \\
\mathcal{Z}_{n l ; \beta, \gamma, K_{Z}}(t) & =\mathrm{J} n l\left(K_{Z} z, \Phi^{\prime} ; t\right)+\dot{z}(t)-\mathrm{J}_{n l}(L ; t) z(t) \\
& =K_{Z} z(t) \Phi^{\prime}\left(\xi_{n l}(t)\right)+\dot{z}(t)-g^{\prime}\left(\Phi\left(\xi_{n l}(t)\right)\right) z(t) .
\end{aligned}
$$

Remembering the choice $\dot{Z}(t)=K_{Z} z(t)$ and suppressing the dependence on $\beta, \gamma$ and $K_{Z}$, the expression (5.137) can now be written as

$$
\begin{aligned}
\mathcal{J}^{-}(t)=-\Theta(t)-\mathcal{Z}(t) & \\
+ & \mathcal{R}_{1}\left(\pi^{\diamond} \theta, \pi^{\diamond \diamond} \theta, z ; t\right)+\mathcal{R}_{2}\left(\dot{Z}, \pi^{\diamond} \theta, \pi^{\diamond \diamond} \theta ; t\right)+\mathcal{R}_{3}\left(\dot{\theta}, \pi^{\diamond \diamond} \theta ; t\right) \\
& \quad+\mathcal{R}_{4}\left(\pi^{\diamond} \dot{\theta}, \pi^{\diamond} \theta ; t\right)+\mathcal{R}_{5}\left(\pi^{\diamond} \theta, \pi^{\diamond \diamond} \theta, \pi^{\diamond \diamond \diamond} \theta ; t\right)+\mathcal{R}_{6}\left(\dot{\theta}, \pi^{\diamond} \theta ; t\right)+\mathcal{R}_{7}\left(\pi^{\diamond} \theta ; t\right) \\
+ & \mathcal{E}_{1}\left(\pi^{\diamond \diamond} \dot{\theta} ; t\right)+\mathcal{E}_{2}\left(\pi^{\diamond \diamond \diamond} \theta ; t\right)+\mathcal{E}_{3}\left(\pi^{\diamond} \dot{\theta}, \pi^{\diamond \diamond} \theta ; t\right) .
\end{aligned}
$$

The following two results concern the terms $\Theta(t)$ and $\mathcal{Z}(t)$, which are the ones for which a definite sign is (almost) available. We note that the strictly positive function $\mathcal{Q}(t)$ was defined in (5.223).

Lemma 5.27. Pick any $\delta_{\omega}>0$, any $\beta>1$ and any $\epsilon_{3}>0$. Then there exist $\gamma_{*}=\gamma_{*}\left(\delta_{\omega}, \beta, \epsilon_{3}\right) \geq 1$ such that for any $\gamma \geq \gamma_{*}$, any $t \geq 1$ and any $l \in \mathbb{Z}$, the bound

$$
\left|\times_{l ; \beta, \gamma}(t)-\mathcal{Q}_{l ; \beta, \gamma}(t) \Phi^{\prime}\left(\xi_{n l}(t)\right)\right| \leq \frac{1}{26} \eta_{z} \epsilon_{3} t^{-3 / 2}
$$

holds provided $|\rho(l, \gamma ; t)| \leq \delta_{\omega}$, while

$$
\left|\times_{l ; \beta, \gamma}(t)\right| \leq \frac{1}{26} \eta_{z} \epsilon_{3} t^{-3 / 2}
$$

holds provided $|\rho(l, \gamma ; t)| \geq \delta_{\omega}$.

Proof. These bounds follow directly from Lemmas 5.16 and 5.20, noting that for any $C^{\prime}>1$ we can choose $\gamma_{*} \geq 1$ in such a way that

$$
\beta \gamma C^{\prime}(\gamma t)^{1 / 2} e^{-\nu_{2} \delta_{\omega}^{2} \gamma t} \leq \beta C^{\prime}(\gamma t)^{-3 / 2} \leq \epsilon_{3} t^{-3 / 2}
$$

holds for any $t \geq 1$ and any $\gamma \geq \gamma_{*}$. 
Lemma 5.28. There exist $\eta_{z}>0$ and $K_{Z}>1$ such that for every $C^{1}$-smooth function $z:[1, \infty) \rightarrow \mathbb{R}$ that has $z(t)>0$ and $\dot{z}(t) \geq-\eta_{z} z(t)$ for all $t \geq 1$, we have

$$
\mathcal{Z}_{n l ; \beta, \gamma, K_{Z}}(t) \geq \eta_{z} z(t)
$$

for all $\beta \geq 1$, all $\gamma \geq 1$, all $(n, l) \in \mathbb{Z}^{2}$ and all $t \geq 1$.

Proof. Since

$$
\mathcal{Z}_{n l ; \beta, \gamma, K_{Z}} \geq\left[K_{Z} \Phi^{\prime}\left(\xi_{n l}(t)\right)-\eta_{z}-g^{\prime}\left(\Phi\left(\xi_{n l}(t)\right)\right)\right] z(t),
$$

it suffices to choose $\eta_{z}>0$ and $K_{Z} \gg 1$ in such a way that

$$
K_{Z} \Phi^{\prime}(\xi)-\eta_{z}-D(\Phi(\xi)) \geq \eta_{z}
$$

holds for all $\xi \in \mathbb{R}$. This is possible because of the limits $\Phi(-\infty)=0, \Phi(+\infty)=1$, the inequalities $g^{\prime}(0)<0$ and $g^{\prime}(1)<0$ and the fact that $\Phi^{\prime}(\xi)>0$ for all $\xi \in \mathbb{R}$.

We are now ready to estimate the numbered terms appearing in (5.254). The terms $\mathcal{R}_{1}$ and $\mathcal{R}_{7}$ need to be considered separately in the near-field and far-field regimes, but the remaining numbered terms can be handled using global bounds.

Lemma 5.29. There exists $\delta_{z}>0$ such that the following is true. Pick any $\delta_{\omega}>0$, any $\beta>1$ and any $\epsilon_{3}>0$. Then there exist $\gamma_{*}=\gamma_{*}\left(\delta_{\omega}, \beta, \epsilon_{3}\right) \geq 1$ such that for any function $z:[1, \infty) \rightarrow\left(0, \delta_{z}\right]$, any $\gamma \geq \gamma_{*}$, any $t \geq 1$ and any $l \in \mathbb{Z}$, we have the bounds

$$
\begin{aligned}
\left|\left[\mathcal{R}_{1}\left(\pi^{\diamond} \theta, \pi^{\diamond} \theta, z ; t\right)\right]_{n l}\right| \leq & \frac{1}{26} \eta_{z}|z(t)|+\frac{1}{26} \eta_{z} \epsilon_{3} t^{-3 / 2} \\
& \quad+\frac{1}{2} \mathcal{Q}_{l ; \beta, \gamma, t_{*}}(t) \Phi^{\prime}\left(\xi_{n l}(t)\right), \\
\left|\left[\mathcal{R}_{7}\left(\pi^{\diamond} \theta ; t\right)\right]_{n l}\right| \leq & \frac{1}{2} \mathcal{Q}_{l ; \beta, \gamma, t_{*}}(t) \Phi^{\prime}\left(\xi_{n l}(t)\right)+\frac{1}{26} \eta_{z} \epsilon_{3} t^{-3 / 2},
\end{aligned}
$$

provided $|\rho(l, \gamma ; t)| \leq \delta_{\omega}$, together with the bounds

$$
\begin{aligned}
& \left|\left[\mathcal{R}_{1}\left(\pi^{\diamond} \theta, \pi^{\diamond \diamond} \theta, z ; t\right)\right]_{n l}\right| \leq \frac{1}{26} \eta_{z}|z(t)|+\frac{1}{26} \eta_{z} \epsilon_{3} t^{-3 / 2}, \\
& \left|\left[\mathcal{R}_{7}\left(\pi^{\diamond} \theta ; t\right)\right]_{n l}\right|
\end{aligned}
$$

provided $|\rho(l, t, \gamma)| \geq \delta_{\omega}$. Here we have used $\theta=\theta_{; \beta, \gamma}$.

Proof. First of all, pick any $0<\kappa^{\prime} \leq 1$. Possibly decreasing $\delta_{z}>0$ and increasing $\gamma_{*} \geq 1$, Lemma 5.25 shows that we can arrange for

$$
\delta_{z}+\left|\pi_{l}^{\diamond} \theta_{; \beta, \gamma}(t)\right|+\left|\pi_{l}^{\diamond} \theta_{; \beta, \gamma}(t)\right| \leq \kappa^{\prime} \leq 1, \quad \gamma \geq \gamma_{*}, \quad l \in \mathbb{Z}, \quad t \geq 1 .
$$

Recalling the constant $C_{1}=C_{1}(1)$ defined in Lemma 5.6, we see that in the near field $|\rho| \leq \delta_{\omega}$ we have

$$
\begin{aligned}
\left|\left[\mathcal{R}_{1}\left(\pi^{\diamond} \theta, \pi^{\diamond \diamond} \theta, z ; t\right)\right]_{n l}\right| \leq & C_{1}|z(t)|\left[|z(t)|+\pi_{l}^{\diamond} \theta(t)+\left|\pi_{l}^{\diamond \diamond} \theta(t)\right|\right] \\
& +C_{1}\left(\left|\pi_{l}^{\diamond} \theta(t)\right|+\left|\pi_{l}^{\diamond \diamond} \theta(t)\right|\right)^{2} \Phi^{\prime}\left(\xi_{n l}(t)\right) \\
\leq \quad & C_{1} \kappa^{\prime}|z(t)| \\
& \quad+16 \beta^{2} t^{-2 \alpha} C_{1} C_{4}^{2}\left[\left(\rho^{2}+(\gamma t)^{-2}\right) v_{l ; \gamma}^{2}(t)+(\gamma t) e^{-2 \nu_{2} \delta_{\omega}^{2} \gamma t}\right] \Phi^{\prime}\left(\xi_{n l}(t)\right) .
\end{aligned}
$$

In view of the definition (5.223) for $\mathcal{Q}_{l ; \beta, \gamma}(t)$, it hence suffices to pick $\kappa^{\prime}>0$ sufficiently small and $\gamma_{*} \geq 1$ sufficiently large to ensure that

$$
C_{1} \kappa^{\prime} \leq \frac{1}{26} \eta_{z}, \quad 16 C_{1} \beta C_{4}^{2} \leq \frac{1}{16} \nu_{2} \gamma_{*}, \quad 16 C_{1} \beta C_{4}^{2} \gamma_{*}^{-1} \leq \frac{1}{16}
$$


all hold, together with an exponential estimate similar to (5.257).

The far field case $|\rho(l, t ; \gamma)| \geq \delta_{\omega}$ can be treated in a similar fashion. Finally, inspection of (5.6) shows that $\mathcal{R}_{7}$ only contains terms that are also present in $\mathcal{R}_{1}$.

Lemma 5.30. Pick any $\beta>1$ and $\epsilon_{3}>0$. Then there exists $\gamma_{*}=\gamma_{*}\left(\beta, \epsilon_{3}\right) \geq 1$ such that for any function $z:[0, \infty) \rightarrow(0,1]$, any $\gamma \geq \gamma_{*}$ and any $t \geq 1$, the following bounds hold for every $(n, l) \in \mathbb{Z}^{2}$,

$$
\begin{aligned}
& \left|\left[\mathcal{R}_{2}\left(K_{Z} z, \pi^{\diamond} \theta, \pi^{\diamond} \theta ; t\right)\right]_{n l}\right| \leq \frac{1}{26} \eta_{z} z(t), \\
& \left|\left[\mathcal{R}_{3}\left(\dot{\theta}, \pi^{\diamond \diamond} \theta ; t\right)\right]_{n l}\right| \\
& \left|\left[\mathcal{R}_{4}\left(\pi^{\diamond} \dot{\theta}, \pi^{\diamond} \theta ; t\right)\right]_{n l}\right| \\
& \left|\left[\mathcal{R}_{5}\left(\pi^{\diamond} \theta, \pi^{\diamond} \theta, \pi^{\diamond \diamond \diamond} \theta ; t\right)\right]_{n l}\right| \leq \frac{1}{26} \eta_{3} \eta^{-3 / 2} \epsilon_{3} t^{-3 / 2}, \\
& \left|\left[\mathcal{R}_{6}\left(\dot{\theta}, \pi^{\diamond} \theta ; t\right)\right]_{n l}\right|
\end{aligned}
$$

in which we used $\theta=\theta_{; \beta, \gamma}$.

Proof. First note that the global bounds in Lemma 5.21 imply that

$$
\left|\left[\mathcal{R}_{2}\left(K_{Z} z, \pi^{\diamond} \theta, \pi^{\diamond} \theta ; t\right)\right]_{n l}\right| \leq \beta t^{-\alpha} C_{1} K_{Z}|z(t)|\left[C_{6}(\gamma t)^{-1 / 2}+C_{6}(\gamma t)^{-1}\right]
$$

so for the first inequality it suffices to pick $\gamma_{*} \geq 1$ in such a way that

$$
\beta C_{1} K_{Z} C_{6}\left(\gamma_{*}^{-1 / 2}+\gamma_{*}^{-1}\right) \leq \frac{1}{26} \eta_{z} .
$$

In view of the global bounds obtained in Lemmas 5.21, 5.22 and 5.23, the remaining estimates follow from the computations

$$
\begin{aligned}
\left|\left[\mathcal{R}_{3}\left(\dot{\theta}, \pi^{\diamond \diamond} \theta ; t\right)\right]_{n l}\right| & \leq C_{1}\left|\dot{\theta}_{l}(t)\right|\left|\pi_{l}^{\diamond \diamond} \theta(t)\right| \\
& \leq C_{1} \beta^{2} C_{6}^{2}\left[(\gamma t)^{-1 / 2}+\gamma(\gamma t)^{-1}\right](\gamma t)^{-1}, \\
\left|\left[\mathcal{R}_{4}\left(\pi^{\diamond} \dot{\theta}, \pi^{\diamond} \theta ; t\right)\right]_{n l}\right| & \leq C_{1}\left|\pi_{l}^{\diamond} \dot{\theta}(t)\right|\left|\pi_{l}^{\diamond} \theta(t)\right| \\
& \leq C_{1} \beta^{2} C_{6}^{2}\left[\gamma(\gamma t)^{-3 / 2}+(\gamma t)^{-1}\right](\gamma t)^{-1 / 2}, \\
\left|\left[\mathcal{R}_{5}\left(\pi^{\diamond} \theta, \pi^{\diamond \diamond} \theta, \pi^{\diamond \diamond \diamond} \theta ; t\right)\right]_{n l}\right| \leq & \left|\pi_{l}^{\diamond} \theta(t)\right|\left[\left|\pi_{l}^{\diamond} \theta(t)\right|+\left|\pi_{l}^{\diamond} \theta(t)\right|^{2}+\left|\pi_{l}^{\diamond \diamond \diamond} \theta(t)\right|\right]+\left|\pi_{l}^{\diamond \diamond} \theta(t)\right|^{2} \\
\leq & C_{1} \beta^{2} C_{6}^{2}(\gamma t)^{-1 / 2}\left[(\gamma t)^{-1}+(\gamma t)^{-1}+(\gamma t)^{-3 / 2}\right] \\
& \quad+C_{1} \beta^{2} C_{6}^{2}(\gamma t)^{-2}, \\
\left|\left[\mathcal{R}_{6}\left(\dot{\theta}, \pi^{\diamond} \theta ; t\right)\right]_{n l}\right| & \leq C_{1}\left|\dot{\theta}_{l}(t)-\alpha_{\mu}^{\diamond} \pi_{l ; \mu}^{\diamond} \theta(t)\right|\left|\pi_{l}^{\diamond} \theta(t)\right| \\
& \leq C_{1} \beta^{2} C_{6}^{2}(\gamma t)^{-1}(\gamma t)^{-1 / 2} .
\end{aligned}
$$

Indeed, the worst of these terms is given by $C_{1} \beta^{2} C_{6}^{2}\left[(\gamma t)^{-3 / 2}+\gamma(\gamma t)^{-2}\right]$, which can easily be estimated by $\epsilon_{3} t^{-3 / 2}$ for all $t \geq 1$ by picking $\gamma_{*}$ sufficiently large.

Lemma 5.31. Pick any $\beta>1$ and $\epsilon_{3}>0$. Then there exists $\gamma_{*}=\gamma_{*}\left(\beta, \epsilon_{3}\right)$, such that for any $\gamma \geq \gamma_{*}$, any $t \geq 1$ and any $(n, l) \in \mathbb{Z}^{2}$, we have the bounds

$$
\begin{aligned}
& \left|\left[\mathcal{E}_{1}\left(\pi^{\diamond \diamond} \dot{\theta} ; t\right)\right]_{n l}\right| \leq \frac{1}{26} \eta_{z} \epsilon_{3} t^{-3 / 2}, \\
& \left|\left[\mathcal{E}_{2}\left(\pi^{\diamond \diamond \diamond} \theta ; t\right)\right]_{n l}\right| \leq \frac{1}{26} \eta_{z} \epsilon_{3} t^{-3 / 2} \\
& \left|\left[\mathcal{E}_{3}\left(\pi^{\diamond} \dot{\theta}, \pi^{\diamond \diamond} \theta ; t\right)\right]_{n l}\right| \leq \frac{1}{26} \eta_{z} \epsilon_{3} t^{-3 / 2} .
\end{aligned}
$$


Proof. Combining the global bounds obtained in Lemmas 5.21, 5.22 and 5.23 with the estimates in Lemma 5.8, we compute

$$
\begin{aligned}
\left|\left[\mathcal{E}_{1}\left(\pi^{\diamond \diamond} \dot{\theta} ; t\right)\right]_{n l}\right| & \leq C_{1}\left|\pi_{l}^{\diamond \diamond} \dot{\theta}(t)\right| \\
& \leq C_{1} C_{6} \beta\left[\gamma(\gamma t)^{-2}+(\gamma t)^{-3 / 2}\right], \\
\left|\left[\mathcal{E}_{2}\left(\pi^{\diamond \diamond \diamond} \theta ; t\right)\right]_{n l}\right| & \leq C_{1}\left|\pi_{l}^{\diamond \diamond \diamond} \theta(t)\right| \\
& \leq C_{1} C_{6} \beta(\gamma t)^{-3 / 2}, \\
\left|\left[\mathcal{E}_{3}\left(\pi^{\diamond} \dot{\theta}, \pi^{\diamond \diamond} \theta ; t\right)\right]_{n l}\right| & \leq C_{1}\left|\pi_{l}^{\diamond}\left[\dot{\theta}(t)-\alpha_{\mu}^{\diamond} \pi_{l ; \mu}^{\diamond} \theta(t)\right]\right| \\
& \leq C_{1} C_{6} \beta \gamma(\gamma t)^{-3 / 2} .
\end{aligned}
$$

The worst term is hence $C_{1} C_{6} \beta \gamma(\gamma t)^{-3 / 2}$, so it suffices to pick $\gamma_{*}$ in such a way that

$$
2 C_{1} C_{6} \beta \gamma_{*}^{-1 / 2} \leq \frac{1}{26} \eta_{z} \epsilon_{3} .
$$

Proof of Proposition 5.24. The statements follow from the discussion above, picking $\delta_{z}>0$ and $\gamma \geq \gamma_{*} \geq 1$ in such a way that the statements in Lemmas 5.27, 5.29, 5.30 and 5.31 all hold. In particular, these estimates imply that for any $(n, l) \in \mathbb{Z}^{2}$ and any $t \geq 1$ we have

$$
\mathcal{J}_{n l}^{-}(t) \leq-\frac{1}{2} \eta_{z} z(t)<0,
$$

as desired.

Proof of Proposition 5.1. The statements concerning $W^{-}$follow directly from Proposition 5.24, upon choosing $\beta>1$ to be sufficiently large and writing

$$
W_{n l}^{-}(t)=u_{n l}^{-}(t-1) \text {. }
$$

We stress that the function $\theta_{; \beta, \gamma}$ depends only on the parameters $\epsilon_{1}, \epsilon_{2}, \epsilon_{3}, \Omega_{\perp}$ and $\Omega_{\text {phase }}$ and not on the specific form of $z(t)$. The super-solution $W^{+}$can be constructed analogously.

\section{The Entire Solution}

Throughout the remainder of this paper we focus our attention on the obstructed LDE (6.6). The purpose of this section is to establish the existence of an entire solution to (6.6) that converges to a planar travelling wave as $t \rightarrow-\infty$. The ideas here closely follow the presentation in $[9, \S 2-\S 3]$, but the discreteness of the lattice requires certain technical adjustments.

Throughout this section we fix a pair $\left(\sigma_{h}, \sigma_{v}\right) \in \mathbb{Z}^{2} \backslash\{0,0\}$ with $\operatorname{gcd}\left(\sigma_{h}, \sigma_{v}\right)=1$ and recall the notation $\sigma=\max \left\{\left|\sigma_{h}\right|,\left|\sigma_{v}\right|\right\}$. For any $S \subset \mathbb{Z}^{2}$, we introduce the neighbour set

$$
\mathcal{N}_{S}^{\times}(n, l)=\left\{\left(n+\sigma_{h}, l+\sigma_{v}\right),\left(n+\sigma_{v}, l-\sigma_{h}\right),\left(n-\sigma_{h}, l-\sigma_{v}\right),\left(n-\sigma_{v}, l+\sigma_{h}\right)\right\} \cap S,
$$

together with the associated punctured Laplacian

$$
\left[\Delta_{S}^{\times} v\right]_{n l}=\sum_{\left(n^{\prime}, l^{\prime}\right) \in \mathcal{N}_{S}^{\times}(n, l)}\left[v_{n^{\prime} l^{\prime}}-v_{n l}\right]
$$

and the boundary

$$
\partial_{\times} S=\left\{(n, l) \in S \mid \mathcal{N}_{S}^{\times}(n, l) \neq \mathcal{N}_{\mathbb{Z}^{2}}^{\times}(n, l)\right\}
$$


Upon writing

$$
K_{\mathrm{obs}}^{\times}=\left\{(n, l) \in \mathbb{Z}^{2} \text { for which }(n, l)=\left(i \sigma_{h}+j \sigma_{v}, i \sigma_{v}-j \sigma_{h}\right) \text { for some }(i, j) \in K_{\mathrm{obs}}\right\},
$$

together with

$$
\Lambda^{\times}=\mathbb{Z}^{2} \backslash K_{\text {obs }}^{\times}
$$

we see that the obstructed LDE (2.31) is transformed into

$$
\dot{u}_{n l}(t)=\left[\Delta_{\Lambda^{\times}}^{\times} u(t)\right]_{n l}+g\left(u_{n l}(t)\right), \quad(n, l) \in \Lambda^{\times} .
$$

Proposition 6.1. Consider the obstructed LDE (6.6) and assume that (Hg), (HK1) and $(h \Phi)_{\S 3}$ with $c>0$ all hold. Then there exists a $C^{1}$-smooth function $U: \mathbb{R} \rightarrow \ell^{\infty}\left(\Lambda^{\times} ; \mathbb{R}\right)$ that satisfies the obstructed $L D E$ (6.6) for all $t \in \mathbb{R}$, admits the uniform limit

$$
\sup _{(n, l) \in \Lambda^{\times}}\left|U_{n l}(t)-\Phi(n+c t)\right| \rightarrow 0, \quad t \rightarrow-\infty
$$

and enjoys the estimates

$$
0<U_{n l}(t)<1, \quad \dot{U}_{n l}(t)>0
$$

for all $(n, l) \in \Lambda^{\times}$and $t \in \mathbb{R}$. In addition, any $C^{1}$-smooth function $V: \mathbb{R} \rightarrow \ell^{\infty}\left(\Lambda^{\times} ; \mathbb{R}\right)$ that satisfies (6.6) for all $t \in \mathbb{R}$ together with (6.7) must also have $V=U$.

By relabelling our coordinate system and shifting the wave profile $\Phi$, we can arrange for the following two conditions to hold.

$(\mathrm{hK})_{\S 6}$ The obstacle satisfies $K_{\mathrm{obs}}^{\times} \subset\{n<-2 \sigma\} \subset \mathbb{Z}^{2}$.

$(\mathrm{h} \Phi)_{\S 6}$ Recalling the inequality $g(\xi) \leq 0$ for $0 \leq \xi \leq a$, we have $\Phi(0) \leq a$. In addition, we have $\Phi^{\prime \prime}(\xi)>0$ for all $\xi \leq 0$, together with $c>0$.

Upon recalling the exponents $\left(\kappa_{\Phi}, \eta_{\Phi}^{ \pm}\right)$defined in Lemma 3.4, we fix the exponent

$$
\eta_{0}=\min \left\{\eta_{\Phi}^{-}, \kappa_{\Phi}\right\}
$$

For any $M_{0}>1$, we now introduce the function

$$
\Xi(t)=\Xi_{M_{0}}(t)
$$

that is uniquely defined by the initial value problem

$$
\dot{\Xi}(t)=M_{0} e^{\eta_{0}(c t+\Xi(t))}, \quad \Xi(-\infty)=0 .
$$

We note that $\Xi(t)$ is defined on the interval $\left(-\infty,-T_{0}\right)$, for some $T_{0}=T_{0}\left(M_{0}\right) \gg 1$.

Our main task in this section is to show that the two $C^{1}$-smooth functions $u^{ \pm}:\left(-\infty,-T_{0}\left(M_{0}\right)\right) \rightarrow$ $\ell^{\infty}\left(\mathbb{Z}^{2} ; \mathbb{R}\right)$ defined by

$$
\begin{aligned}
& u_{n l}^{-}(t)= \begin{cases}\Phi(n+c t-\Xi(t))-\Phi(-n+c t-\Xi(t)) & n \geq 0, \\
0 & n<0,\end{cases} \\
& u_{n l}^{+}(t)= \begin{cases}\Phi(n+c t+\Xi(t))+\Phi(-n+c t+\Xi(t)) & n \geq 0, \\
2 \Phi(c t+\Xi(t)) & n<0,\end{cases}
\end{aligned}
$$

are sub respectively super-solutions for (6.6). The form of these two functions is precisely the same as that of their counterparts from [9], but the non-local terms in our LDE require special care in 
our analysis because the $\{n<0\}$ and $\{n \geq 0\}$ regimes interact with each other across the $\{n=0\}$ boundary.

In order to understand these non-local terms, we first notice that $(\mathrm{hK})_{\S 6}$ implies that

$$
\Delta_{\Lambda \times}^{\times} u^{ \pm}(t)=\Delta^{\times} u^{ \pm}(t)
$$

for all $t \leq-T_{0}\left(M_{0}\right)$. In addition, we introduce the notation

$$
\begin{array}{r}
\left.\mathcal{I}_{\Delta}^{-}(t)\right]_{n}=c \Phi^{\prime}(n+c t-\Xi(t))-c \Phi^{\prime}(-n+c t-\Xi(t)) \\
-g(\Phi(n+c t-\Xi(t)))+g(\Phi(-n+c t-\Xi(t))), \\
\left.\mathcal{I}_{\Delta}^{+}(t)\right]_{n}=c \Phi^{\prime}(n+c t+\Xi(t))+c \Phi^{\prime}(-n+c t+\Xi(t)) \\
\quad-g(\Phi(n+c t+\Xi(t)))-g(\Phi(-n+c t+\Xi(t))) .
\end{array}
$$

Before we state our first two technical results concerning the discrete Laplacians (6.13), we recall the notation

$$
\left(\sigma_{1}, \ldots, \sigma_{5}\right)=\left(\sigma_{v},-\sigma_{h},-\sigma_{v}, \sigma_{h}, 0\right)
$$

together with the vector

$$
\left(L_{1}^{\times}, \ldots, L_{5}^{\times}\right)=(1,1,1,1,-4) \in \mathbb{R}^{5}
$$

and the summation convention introduced in $\S 5.1$.

Lemma 6.2. Consider the obstructed LDE (6.6), suppose that $(H g),(h K)_{\S 6},(h \Phi)_{\S 3}$ and $(h \Phi)_{\S 6}$ all hold and pick any $M_{0}>1$. Then for any $t \leq-T_{0}\left(M_{0}\right)$, we have the inequalities

$$
\begin{array}{lll}
{\left[\Delta^{\times} u^{-}(t)\right]_{n l} \geq\left[\mathcal{I}_{\Delta}^{-}(t)\right]_{n},} & & n>0, \\
{\left[\Delta^{\times} u^{-}(t)\right]_{n l} \geq 0,} & n \leq 0 .
\end{array}
$$

Proof. For $n \geq \sigma$, we may compute

$$
\begin{aligned}
{\left[\Delta^{\times} u^{-}(t)\right]_{n l} } & =L_{\mu}^{\times} \Phi\left(n+\sigma_{\mu}+c t-\Xi(t)\right)-L_{\mu}^{\times} \Phi\left(-n-\sigma_{\mu}+c t-\Xi(t)\right) \\
& =\left[\mathcal{I}_{\Delta}^{-}(t)\right]_{n} .
\end{aligned}
$$

For $0<n<\sigma$, we have

$$
\begin{aligned}
{\left[\Delta^{\times} u^{-}(t)\right]_{n l}-\left[\mathcal{I}_{\Delta}^{-}(t)\right]_{n} } & =\sum_{n+\sigma_{\mu}<0}\left[\Phi\left(-n-\sigma_{\mu}+c t-\Xi(t)\right)-\Phi\left(n+\sigma_{\mu}+c t-\Xi(t)\right)\right] \\
& \geq 0
\end{aligned}
$$

since $\Phi$ is strictly increasing. Similarly, for $-\sigma<n \leq 0$ we have

$$
\begin{aligned}
{\left[\Delta^{\times} u^{-}(t)\right]_{n l} } & =\sum_{n+\sigma_{\mu}>0}\left[\Phi\left(n+\sigma_{\mu}+c t-\Xi(t)\right)-\Phi\left(-n-\sigma_{\mu}+c t-\Xi(t)\right)\right] \\
& \geq 0,
\end{aligned}
$$

while for $n \leq-\sigma$ we have $\left[\Delta^{\times} u^{-}(t)\right]_{n l}=0$.

Lemma 6.3. Consider the LDE (6.6) and suppose that $(H g),(h K)_{\S 6},(h \Phi)_{\S 3}$ and $(h \Phi)_{\S 6}$ all hold. There exists a constant $C_{1}>1$ so that for any $M_{0}>1$ and any $t \leq-T_{0}\left(M_{0}\right)$ for which ct $+\Xi(t) \leq$ $-\sigma$, the estimate

$$
\left[\Delta^{\times} u^{+}(t)\right]_{n l}-\left[\mathcal{I}_{\Delta}^{+}(t)\right]_{n} \leq C_{1} e^{-\left(\eta_{\Phi}^{-}+\kappa_{\Phi}\right)|c t+\Xi(t)|} \mathbf{1}_{n \in[0, \sigma)}
$$

holds whenever $n \geq 0$, while

$$
\left[\Delta^{\times} u^{+}\right]_{n l}-c \Phi^{\prime}(c t+\Xi(t))+g^{\prime}(0) \Phi(c t+\Xi(t)) \leq C_{1} e^{-\left(\eta_{\Phi}^{-}+\kappa_{\Phi}\right)|c t+\Xi(t)|}
$$

holds whenever $n<0$. 
Proof. For convenience, we introduce the shorthand $\xi=c t+\Xi(t)$. For $n \geq \sigma$, we have

$$
\begin{aligned}
{\left[\Delta^{\times} u^{+}(t)\right]_{n l} } & =L_{\mu}^{\times} \Phi\left(n+\sigma_{\mu}+\xi\right)+L_{\mu}^{\times} \Phi\left(-n-\sigma_{\mu}+\xi\right) \\
& =\left[\mathcal{I}_{\Delta}^{+}(t)\right]_{n} .
\end{aligned}
$$

For $0 \leq n<\sigma$, we may use the asymptotics in Proposition 3.4 to compute

$$
\begin{aligned}
{\left[\Delta^{\times} u^{+}(t)\right]_{n l}-\left[\mathcal{I}_{\Delta}^{+}(t)\right]_{n}=} & \sum_{n+\sigma_{\mu}<0}\left[2 \Phi(\xi)-\Phi\left(n+\sigma_{\mu}+\xi\right)-\Phi\left(-n-\sigma_{\mu}+\xi\right)\right] \\
\leq & \sum_{n+\sigma_{\mu}<0} C_{\Phi}^{-} e^{\eta_{\Phi}^{-} \xi}\left[2-e^{\eta_{\Phi}^{-}\left(n+\sigma_{\mu}\right)}-e^{-\eta_{\Phi}^{-}\left(n+\sigma_{\mu}\right)}\right] \\
& \quad+\sum_{n+\sigma_{\mu}<0} K_{\Phi} e^{\left(\eta_{\Phi}^{-}+\kappa_{\Phi}\right) \xi}\left[2+e^{\left(\eta_{\Phi}^{-}+\kappa_{\Phi}\right)\left(n+\sigma_{\mu}\right)}+e^{-\left(\eta_{\Phi}^{-}+\kappa_{\Phi}\right)\left(n+\sigma_{\mu}\right)}\right] \\
& \leq \sum_{n+\sigma_{\mu}<0} K_{\Phi} e^{\left(\eta_{\Phi}^{-}+\kappa_{\Phi}\right) \xi}\left[2+2 e^{\left.\left(\eta_{\Phi}^{-}+\kappa_{\Phi}\right) \sigma\right)}\right],
\end{aligned}
$$

since $2-2 \cosh \left(\eta_{\Phi}^{-}\left(n+\sigma_{\mu}\right)\right) \leq 0$. These two observations readily yield the first estimate (6.21).

For $-\sigma \leq n<0$ we obtain

$$
\left[\Delta^{\times} u^{+}(t)\right]_{n l}=\sum_{n+\sigma_{\mu} \geq 0}\left[\Phi\left(n+\sigma_{\mu}+\xi\right)+\Phi\left(-n-\sigma_{\mu}+\xi\right)-2 \Phi(\xi)\right] .
$$

In particular, we may write

$$
Q_{n l}(t)=\left[\Delta^{\times} u^{+}(t)\right]_{n l}-c \Phi^{\prime}(\xi)+g^{\prime}(0) \Phi(\xi)
$$

and compute

$$
\begin{aligned}
Q_{n l}(t) \leq C_{\Phi}^{-} e^{\eta_{\Phi}^{-} \xi} \sum_{n+\sigma_{\mu} \geq 0}\left[e^{\eta_{\Phi}^{-}\left(n+\sigma_{\mu}\right)}+e^{-\eta_{\Phi}^{-}\left(n+\sigma_{\mu}\right)}-2\right] \\
\quad-c C_{\Phi}^{-} \eta_{\Phi}^{-} e^{\eta_{\Phi}^{-} \xi}+g^{\prime}(0) C_{\Phi}^{-} e^{\eta_{\Phi}^{-} \xi} \\
+K_{\Phi} e^{\left(\eta_{\Phi}^{-}+\kappa_{\Phi}\right) \xi} \sum_{n+\sigma_{\mu} \geq 0}\left[e^{\left(\eta_{\Phi}^{-}+\kappa_{\Phi}\right)\left(n+\sigma_{\mu}\right)}+e^{-\left(\eta_{\Phi}^{-}+\kappa_{\Phi}\right)\left(n+\sigma_{\mu}\right)}+2\right] \\
\quad+K_{\Phi} e^{\left(\eta_{\Phi}^{-}+\kappa_{\Phi}\right) \xi}\left[c+\left|g^{\prime}(0)\right|\right] \\
\leq \quad C_{\Phi}^{-} e^{\eta_{\Phi}^{-} \xi}\left[2 \cosh \left(\sigma_{h} \eta_{\Phi}^{-}\right)+2 \cosh \left(\sigma_{v} \eta_{\Phi}^{-}\right)-4-c \eta_{\Phi}^{-}+g^{\prime}(0)\right] \\
\quad+K_{\Phi} e^{\left(\eta_{\Phi}^{-}+\kappa_{\Phi}\right) \xi}\left[c+\left|g^{\prime}(0)\right|+2+2 e^{\left.\left(\eta_{\Phi}^{-}+\kappa_{\Phi}\right) \sigma\right)}\right] \\
=K_{\Phi} e^{\left(\eta_{\Phi}^{-}+\kappa_{\Phi}\right) \xi}\left[c+\left|g^{\prime}(0)\right|+2+2 e^{\left.\left(\eta_{\Phi}^{-}+\kappa_{\Phi}\right) \sigma\right)}\right],
\end{aligned}
$$

where the last equality follows from (3.13). Finally, for $n<-\sigma$ we have $\left[\Delta^{\times} u^{+}(t)\right]_{n l}=0$, which establishes (6.22) and concludes the proof.

The specific forms for $u^{ \pm}$suggest that it is worthwhile to introduce the two auxilliary functions

$$
\begin{aligned}
& G(n, \xi)=g(\Phi(n+\xi))+g(\Phi(-n+\xi))-g(\Phi(n+\xi)+\Phi(-n+\xi)), \\
& H(n, \xi)=g(\Phi(n+\xi))-g(\Phi(-n+\xi))-g(\Phi(n+\xi)-\Phi(-n+\xi)) .
\end{aligned}
$$

The next result collects some useful properties for $G$ and $H$.

Lemma 6.4. Consider the LDE (6.6) and suppose that $(H g),(h \Phi)_{\S 3}$ and $(h \Phi)_{\S 6}$ all hold. Then there exists $C_{2}>1$ such that for every $\xi \in \mathbb{R}$ and $n \in \mathbb{Z}$, we have the inequalities

$$
\begin{aligned}
|G(n, \xi)| & \leq C_{2} \Phi(n+\xi) \Phi(-n+\xi), \\
|H(n, \xi)| & \leq C_{2} \Phi(-n+\xi)(\Phi(n+\xi)-\Phi(-n+\xi)) .
\end{aligned}
$$


In addition, if $\eta_{\Phi}^{-} \leq \eta_{\Phi}^{+}$, there exist constants $L_{2}>1$ and $\kappa_{2}>0$ such that the inequalities

$$
\begin{aligned}
& G(n, \xi) \geq+\kappa_{2} \Phi(-n+\xi), \\
& H(n, \xi) \leq-\kappa_{2} \Phi(-n+\xi)
\end{aligned}
$$

hold for all $\xi \leq 0$ and $n \geq L_{2}-\xi$.

Proof. For any pair $(u, v) \in \mathbb{R}^{2}$ we have

$$
g(u+v)-g(u)-g(v)=u v \int_{0}^{1} \int_{0}^{1} g^{\prime \prime}(s u+t v) d s d t .
$$

In addition, writing $u=\Phi(n+\xi)$ and $v=\Phi(-n+\xi)$, we have

$$
\begin{aligned}
H(n, \xi) & =g(u)-g(v)-g(u-v) \\
& =g((u-v)+v)-g(v)-g(u-v) \\
& =v(u-v) \int_{0}^{1} g^{\prime \prime}(s(u-v)+t v) d s d t .
\end{aligned}
$$

These observations directly imply the estimate (6.29).

We note that the inequalities $c>0$ and $\eta_{\Phi}^{-} \leq \eta_{\Phi}^{+}$directly imply that $g^{\prime}(0)>g^{\prime}(1)$. Upon introducing the quantity

$$
\mathcal{I}_{G}(u, v)=g(v)-g(0)+g(u)-g(u+v)+\left[g^{\prime}(1)-g^{\prime}(0)\right] v,
$$

we may compute

$$
\begin{aligned}
\mathcal{I}_{G}(u, v)= & v \int_{t=0}^{1}\left[g^{\prime}(t v)-g^{\prime}(0)\right] d t-v \int_{t=0}^{1}\left[g^{\prime}(u+t v)-g^{\prime}(1)\right] d t \\
= & v \int_{t=0}^{1}\left[g^{\prime}(t v)-g^{\prime}(0)\right] d t \\
& \quad-v \int_{t=0}^{1}\left[g^{\prime}(u+t v)-g^{\prime}(u)\right] d t-v \int_{t=0}^{1}\left[g^{\prime}(u)-g^{\prime}(1)\right] d t .
\end{aligned}
$$

In particular, there exists $C^{\prime}>1$ for which the bound

$$
\left|\mathcal{I}_{G}(u, v)\right| \leq C^{\prime}|v|[|v|+|1-u|]
$$

holds whenever $|u| \leq 2$ and $|v| \leq 2$. Since $g^{\prime}(0)>g^{\prime}(1)$, we hence see that there exists $\delta^{\prime}>0$ for which $G(n, \xi) \geq \frac{1}{2}\left[g^{\prime}(0)-g^{\prime}(1)\right] \Phi(-n+\xi)$ holds whenever

$$
|\Phi(-n+\xi)|+|1-\Phi(n+\xi)|<\delta^{\prime} .
$$

Noting that the conditions on $(n, \xi)$ in the statement of this result imply that $-n+\xi \leq-L_{2}$ and $n+\xi \geq L_{2}$, one can guarantee (6.36) by picking $L_{2}$ sufficiently large.

Similarly, we write

$$
\begin{aligned}
\mathcal{I}_{H}(u, v) & =g(u)-g(v)-g(u-v)+\left[g^{\prime}(0)-g^{\prime}(1)\right] v \\
& =-\mathcal{I}_{G}(u-v, v),
\end{aligned}
$$

which implies

$$
\left|\mathcal{I}_{H}(u, v)\right| \leq C^{\prime}|v|[2|v|+|1-u|] .
$$

Arguing as above, the estimates (6.30) now easily follow. 
Lemma 6.5. Consider the travelling wave MFDE (3.10) and suppose that $(H g),(h \Phi)_{\S 3}$ and $(h \Phi)_{\S 6}$ all hold. Then there exists a constant $\kappa_{3}>0$ such that we have

$$
\frac{\Phi^{\prime}\left(\xi_{1}\right)-\Phi^{\prime}\left(\xi_{2}\right)}{\Phi\left(\xi_{1}\right)-\Phi\left(\xi_{2}\right)} \geq \kappa_{3}, \quad \xi_{2}<\xi_{1} \leq 0
$$

Proof. Pick any $L^{\prime}>1$ and consider any pair $\left(\xi_{1}, \xi_{2}\right) \in \mathbb{R}^{2}$ for which

$$
\xi_{1}-L^{\prime} \leq \xi_{2}<\xi_{1} \leq 0 .
$$

The mean value theorem now gives

$$
Q\left(\xi_{1}, \xi_{2}\right):=\frac{\Phi^{\prime}\left(\xi_{1}\right)-\Phi^{\prime}\left(\xi_{2}\right)}{\Phi\left(\xi_{1}\right)-\Phi\left(\xi_{2}\right)}=\frac{\left(\xi_{1}-\xi_{2}\right) \Phi^{\prime \prime}\left(\theta_{1}\right)}{\left(\xi_{1}-\xi_{2}\right) \Phi^{\prime}\left(\theta_{2}\right)}=\frac{\Phi^{\prime \prime}\left(\theta_{1}\right)}{\Phi^{\prime}\left(\theta_{2}\right)}>0
$$

for some non-negative pair $\left(\theta_{1}, \theta_{2}\right) \in \mathbb{R}^{2}$ with $\left|\theta_{1}-\theta_{2}\right| \leq L^{\prime}$. The asymptotics (3.17) now imply that there exists a constant $\kappa^{\prime}=\kappa^{\prime}\left(L^{\prime}\right)>0$ so that $Q\left(\xi_{1}, \xi_{2}\right) \geq \kappa^{\prime}\left(L^{\prime}\right)$ whenever (6.40) holds.

On the other hand, the exponential decay of $\Phi^{\prime}$ stated in (3.17) ensures that for $L^{\prime}$ sufficiently large we have

$$
\Phi^{\prime}\left(\xi_{2}\right) \leq \frac{1}{2} \Phi^{\prime}\left(\xi_{1}\right)
$$

whenever $\xi_{2}+L^{\prime} \leq \xi_{1}<0$. The desired lower bound now follows from the estimate

$$
\frac{\Phi^{\prime}\left(\xi_{1}\right)-\Phi^{\prime}\left(\xi_{2}\right)}{\Phi\left(\xi_{1}\right)-\Phi\left(\xi_{2}\right)} \geq \frac{\frac{1}{2} \Phi^{\prime}\left(\xi_{1}\right)}{\Phi\left(\xi_{1}\right)} \geq \kappa^{\prime \prime}
$$

for some $\kappa^{\prime \prime}>0$, again using the asymptotics (3.17) to obtain the second inequality.

We are now ready to verify that the two functions $u^{ \pm}$are a sub and super-solution for (6.6). In view of the preparations above, the following two results can be established almost exactly as in 9 , $\S 2.3]$.

Lemma 6.6. Consider the LDE (6.6) and suppose that $(H g),(h K)_{\S 6},(h \Phi)_{\S 3}$ and $(h \Phi)_{\S 6}$ all hold.

Then there exist $M_{0}>1$ and $T_{*} \geq T_{0}\left(M_{0}\right)$ so that the function $\mathcal{J}^{-}:\left(-\infty,-T_{*}\right] \rightarrow \ell^{\infty}\left(\mathbb{Z}^{2}, \mathbb{R}\right)$ defined by

$$
\mathcal{J}_{n l}^{-}(t)=\dot{u}_{n l}^{-}(t)-\left[\Delta^{\times} u^{-}(t)\right]_{n l}-g\left(u_{n l}^{-}(t)\right)
$$

satisfies the estimate

$$
\mathcal{J}_{n l}^{-}(t) \leq 0, \quad(n, l) \in \mathbb{Z}^{2}, \quad t \leq-T_{*} .
$$

Proof. For $n \leq-\sigma$, we automatically have $\mathcal{J}_{n l}^{-}(t)=0$. For $-\sigma<n \leq 0$, we have

$$
\mathcal{J}_{n l}^{-}(t)=-\left[\Delta^{\times} u^{-}(t)\right]_{n l} \leq 0
$$

by Lemma 6.2 .

For $n>0$ we write

$$
\xi=c t-\Xi(t)
$$

and assume without loss that $\xi<-\sigma$. We compute

$$
\begin{aligned}
\mathcal{J}_{n l}^{-}(t) & =(c-\dot{\Xi}(t))\left[\Phi^{\prime}(\xi+n)-\Phi^{\prime}(\xi-n)\right]-\left[\Delta^{\times} u^{-}(t)\right]_{n l}-g(\Phi(\xi+n)-\Phi(\xi-n)) \\
& =-\dot{\Xi}(t)\left[\Phi^{\prime}(\xi+n)-\Phi^{\prime}(\xi-n)\right]+\left[\mathcal{I}_{\Delta}^{-}(t)\right]_{n}-\left[\Delta^{\times} u^{-}(t)\right]_{n l}+H(n, \xi) \\
& \leq-\dot{\Xi}(t)\left[\Phi^{\prime}(\xi+n)-\Phi^{\prime}(\xi-n)\right]+H(n, \xi) .
\end{aligned}
$$


First, let us consider the case $0<n \leq-\xi$, for which we obviously have $\xi \pm n \leq 0$. In particular, recalling the estimates stated in Corollary 3.5, we may compute

$$
\begin{aligned}
\mathcal{J}_{n l}^{-}(t) & \leq-M_{0} e^{\eta_{0} \xi} e^{2 \eta_{0} \Xi(t)} \kappa_{3}[\Phi(\xi+n)-\Phi(\xi-n)]+C_{2} \Phi(\xi-n)[\Phi(\xi+n)-\Phi(\xi-n)] \\
& \leq\left[-M_{0} e^{\eta_{0} \xi} e^{2 \eta_{0} \Xi(t)} \kappa_{3}+C_{2} \beta_{\text {up }}^{-} e^{-\eta_{\Phi}^{-}|\xi-n|}\right][\Phi(\xi+n)-\Phi(\xi-n)] \\
& =\left[-M_{0} e^{\eta_{0} \xi} e^{2 \eta_{0} \Xi(t)} \kappa_{3}+C_{2} \beta_{\text {up }}^{-} e^{\eta_{\Phi}^{-} \xi} e^{-\eta_{\Phi}^{-} n}\right][\Phi(\xi+n)-\Phi(\xi-n)] \\
& \leq e^{\eta_{0} \xi}\left[-M_{0} \kappa_{3}+C_{2} \beta_{\text {up }}^{-}\right][\Phi(\xi+n)-\Phi(\xi-n)] .
\end{aligned}
$$

By picking $M_{0} \gg 1$ to be sufficiently large we can hence arrange for $\mathcal{J}_{n l}^{-}(t) \leq 0$ to hold in this regime.

We now study the situation that $n>-\xi \geq \sigma$. In this case we have $\xi+n>0$ and $\xi-n<0$, which allows us to estimate

$$
\begin{aligned}
\mathcal{J}_{n l}^{-}(t) & \leq-\dot{\Xi}(t)\left[\Phi^{\prime}(\xi+n)-\Phi^{\prime}(\xi-n)\right]+C_{2} \Phi(\xi-n)[\Phi(\xi+n)-\Phi(\xi-n)] \\
& \leq-M_{0} e^{\eta_{0} \xi} e^{2 \eta_{0} \Xi(t)}\left(\alpha_{\mathrm{low}}^{+} e^{-\eta_{\Phi}^{+}|\xi+n|}-\alpha_{\mathrm{up}}^{-} e^{-\eta_{\Phi}^{-}|\xi-n|}\right)+C_{2} \beta_{\mathrm{up}}^{-} e^{-\eta_{\Phi}^{-}|\xi-n|} \\
& =-M_{0} e^{\eta_{0} \xi} e^{2 \eta_{0} \Xi(t)} e^{-\eta_{\Phi}^{-} n}\left(\alpha_{\mathrm{low}}^{+} e^{\left(\eta_{\Phi}^{-}-\eta_{\Phi}^{+}\right) n} e^{-\eta_{\Phi}^{+} \xi}-\alpha_{\mathrm{up}}^{-} e^{\eta_{\Phi}^{-} \xi}-C_{2} \beta_{\mathrm{up}}^{-} M_{0}^{-1} e^{\left(\eta_{\Phi}^{-}-\eta_{0}\right) \xi} e^{-2 \eta_{0} \Xi(t)}\right) \\
& \leq-M_{0} e^{\eta_{0} \xi} e^{2 \eta_{0} \Xi(t)} e^{-\eta_{\Phi}^{-} n}\left(\alpha_{\mathrm{low}}^{+} e^{\left(\eta_{\Phi}^{-}-\eta_{\Phi}^{+}\right) n} e^{-\eta_{\Phi}^{+} \xi}-\alpha_{\mathrm{up}}^{-} e^{\eta_{\Phi}^{-} \xi}-C_{2} \beta_{\mathrm{up}}^{-} M_{0}^{-1}\right) .
\end{aligned}
$$

If $\eta_{\Phi}^{-} \geq \eta_{\Phi}^{+}$, we obtain the bound

$$
\mathcal{J}_{n l}^{-}(t) \leq-M_{0} e^{\eta_{0} \xi} e^{2 \eta_{0} \Xi(t)} e^{-\eta_{\Phi}^{-} n}\left(\alpha_{\text {low }}^{+} e^{-\eta_{\Phi}^{+} \xi}-\alpha_{\text {up }}^{-} e^{\eta_{\Phi}^{-} \xi}-C_{2} \beta_{\text {up }}^{-} M_{0}^{-1}\right) .
$$

In particular, whenever $\xi \ll-1$ is sufficiently negative to ensure that

$$
\alpha_{\text {low }}^{+} e^{-\eta_{\Phi}^{+} \xi}-\alpha_{\text {up }}^{-} e^{\eta_{\Phi}^{-} \xi}-C_{4} \beta_{\text {up }}^{-} M_{0}^{-1}>0,
$$

we have $\mathcal{J}_{n l}^{-}(t) \leq 0$. This restriction on $\xi$ can be achieved by choosing $T_{*}$ to be sufficiently large.

On the other hand, if $\eta_{\Phi}^{-}<\eta_{\Phi}^{+}$, we consider two separate cases for $n$. In particular, recalling the constants $L_{2}>1$ and $\kappa_{2}$ from Lemma 6.4, we note that for $n \geq-\xi+L_{2}$ we have

$$
\begin{aligned}
\mathcal{J}_{n l}^{-}(t) & \leq \dot{\Xi}(t) \Phi^{\prime}(\xi-n)-\kappa_{2} \Phi(\xi-n) \\
& \leq M_{0} e^{\eta_{0} \xi} e^{2 \eta_{0} \Xi(t)} \alpha_{\mathrm{up}}^{-} e^{-\eta_{\Phi}^{-}|\xi-n|}-\kappa_{2} \beta_{\text {low }}^{-} e^{-\eta_{\Phi}^{-}|\xi-n|} \\
& =e^{-\eta_{\Phi}^{-}|\xi-n|}\left[M_{0} e^{\eta_{0}(c t+\Xi(t))} \alpha_{\text {up }}^{-}-\kappa_{2} \beta_{\text {low }}^{-}\right] .
\end{aligned}
$$

In this case, we have $\mathcal{J}_{n l}^{-}(t) \leq 0$ provided $T_{*}$ is chosen to be sufficiently large to guarantee that $c t+\Xi(t) \ll-1$ is always sufficiently negative to have

$$
M_{0} e^{\eta_{0}(c t+\Xi(t))} \alpha_{\text {up }}^{-}-\kappa_{2} \beta_{\text {low }}^{-} \leq 0, \quad t \leq-T_{*} .
$$

Finally, for $-\xi<n<-\xi+L_{2}$, we see from (6.50) that

$$
\begin{aligned}
\mathcal{J}_{n l}^{-}(t) & \leq-M_{0} e^{\eta_{0} \xi} e^{2 \eta_{0} \Xi(t)} e^{-\eta_{\Phi}^{-} n}\left(\alpha_{\text {low }}^{+} e^{\left(\eta_{\Phi}^{-}-\eta_{\Phi}^{+}\right) n} e^{-\eta_{\Phi}^{+} \xi}-\alpha_{\text {up }}^{-} e^{\eta_{\Phi}^{-} \xi}-C_{2} \beta_{\text {up }}^{-} M_{0}^{-1}\right) \\
& \leq-M_{0} e^{\eta_{0} \xi} e^{2 \eta_{0} \Xi(t)} e^{-\eta_{\Phi}^{-} n}\left(\alpha_{\text {low }}^{+} e^{\left(\eta_{\Phi}^{-}-\eta_{\Phi}^{+}\right)\left(L_{2}-\xi\right)} e^{-\eta_{\Phi}^{+} \xi}-\alpha_{\text {up }}^{-} e^{\eta_{\Phi}^{-} \xi}-C_{2} \beta_{\text {up }}^{-} M_{0}^{-1}\right) \\
& =-M_{0} e^{\eta_{0} \xi} e^{2 \eta_{0} \Xi(t)} e^{-\eta_{\Phi}^{-} n}\left(\alpha_{\text {low }}^{+} e^{\left(\eta_{\Phi}^{-}-\eta_{\Phi}^{+}\right) L_{2}} e^{-\eta_{\Phi}^{-} \xi}-\alpha_{\text {up }}^{-} e^{\eta_{\Phi}^{-} \xi}-C_{2} \beta_{\text {up }}^{-} M_{0}^{-1}\right) .
\end{aligned}
$$

In particular, whenever $\xi \ll-1$ is sufficiently negative to ensure that

$$
\alpha_{\text {low }}^{+} e^{\left(\eta_{\Phi}^{-}-\eta_{\Phi}^{+}\right) L_{2}} e^{-\eta_{\Phi}^{-} \xi}-\alpha_{\text {up }}^{-} e^{\eta_{\Phi}^{-} \xi}-C_{2} \beta_{\text {up }}^{-} M_{0}^{-1} \geq 0,
$$

we have $\mathcal{J}_{n l}^{-}(t) \leq 0$. As before, this restriction on $\xi$ can be achieved by choosing $T_{*}$ to be sufficiently large. 
Lemma 6.7. Consider the LDE (6.6) and suppose that $(\mathrm{Hg}),(h K)_{\S 6},(h \Phi)_{\S 3}$ and $(h \Phi)_{\S 6}$ all hold.

Then there exist $M_{0}>1$ and $T_{*} \geq T_{0}\left(M_{0}\right)$ so that the function $\mathcal{J}^{+}:\left(-\infty,-T_{*}\right] \rightarrow \ell^{\infty}\left(\mathbb{Z}^{2}, \mathbb{R}\right)$ defined by

satisfies the estimate

$$
\mathcal{J}_{n l}^{+}(t)=\dot{u}_{n l}^{+}(t)-\left[\Delta^{\times} u^{+}(t)\right]_{n l}-g\left(u_{n l}^{+}(t)\right)
$$

$$
\mathcal{J}_{n l}^{+}(t) \geq 0, \quad(n, l) \in \mathbb{Z}^{2}, \quad t \leq-T_{*} .
$$

Proof. For convenience, we write

$$
\xi=c t+\Xi(t)
$$

and assume that

$$
\xi<-\sigma, \quad \Phi(\xi)<\frac{a}{2},
$$

which for every $M_{0}>1$ can be arranged by picking $T_{*}=T_{*}\left(M_{0}\right)$ to be sufficiently large. Note in particular that $g(2 \Phi(\xi))<0$.

Choose $C^{\prime}>1$ in such a way that $g^{\prime \prime}(u) \leq C^{\prime}$ for $u \leq 0 \leq 1$. Remembering that $g(0)=0$ and $g^{\prime}(0)<0$, we pick $n<0$ and use Lemma 6.3 to obtain the bound

$$
\begin{aligned}
\mathcal{J}_{n l}^{+}(t) & =2(c+\dot{\Xi}(t)) \Phi^{\prime}(\xi)-\left[\Delta^{\times} u^{+}(t)\right]_{n l}-g(2 \Phi(\xi)) \\
& \geq(c+2 \dot{\Xi}(t)) \Phi^{\prime}(\xi)+g^{\prime}(0) \Phi(\xi)-g(2 \Phi(\xi))-C_{1} e^{\left(\eta_{\Phi}^{-}+\kappa_{\Phi}\right) \xi} \\
& \geq(c+2 \dot{\Xi}(t)) \Phi^{\prime}(\xi)+2 g^{\prime}(0) \Phi(\xi)-g(2 \Phi(\xi))-C_{1} e^{\left(\eta_{\Phi}^{-}+\kappa_{\Phi}\right) \xi} \\
& \geq(c+2 \dot{\Xi}(t)) \Phi^{\prime}(\xi)-2 C^{\prime} \Phi(\xi)^{2}-C_{1} e^{\left(\eta_{\Phi}^{-}+\kappa_{\Phi}\right) \xi} \\
& \geq(c+2 \dot{\Xi}(t)) \Phi^{\prime}(\xi)-2 C^{\prime}\left[\beta_{\mathrm{up}}^{-}\right]^{2} e^{2 \eta_{\Phi}^{-} \xi}-C_{1} e^{\left(\eta_{\Phi}^{-}+\kappa_{\Phi}\right) \xi} \\
& \geq 2 \alpha_{\text {low }}^{-} M_{0} e^{\left(\eta_{0}+\eta_{\Phi}^{-}\right) \xi}-2 C^{\prime}\left[\beta_{\mathrm{up}}^{-}\right]^{2} e^{2 \eta_{\Phi}^{-} \xi}-C_{1} e^{\left(\eta_{\Phi}^{-}+\kappa_{\Phi}\right) \xi} .
\end{aligned}
$$

By picking $M_{0} \gg 1$ to be sufficiently small we can hence guarantee $\mathcal{J}_{n l}^{+}(t) \geq 0$.

Let us now consider $n \geq 0$, for which we may compute

$$
\begin{aligned}
\mathcal{J}_{n l}^{+}(t) & =(c+\dot{\Xi}(t))\left[\Phi^{\prime}(\xi+n)+\Phi^{\prime}(\xi-n)\right]-\left[\Delta^{\times} u^{+}(t)\right]_{n l}-g(\Phi(\xi+n)+\Phi(\xi-n)) \\
& =\dot{\Xi}(t)\left[\Phi^{\prime}(\xi+n)+\Phi^{\prime}(\xi-n)\right]+G(n, \xi)+\left[\mathcal{I}_{\Delta}^{+}(t)\right]_{n}-\left[\Delta^{\times} u^{+}(t)\right]_{n l} \\
& \geq \dot{\Xi}(t)\left[\Phi^{\prime}(\xi+n)+\Phi^{\prime}(\xi-n)\right]+G(n, \xi)-C_{1} e^{-\left(\eta_{\Phi}^{-}+\kappa_{\Phi}\right)|\xi|} \mathbf{1}_{n \in[0, \sigma)} .
\end{aligned}
$$

Restricting attention to $0 \leq n \leq-\xi$, for which we have $\xi \pm n \leq 0$, we may estimate

$$
\begin{aligned}
\mathcal{J}_{n l}^{+}(t) & \geq M_{0} e^{\eta_{0} \xi} \alpha_{\text {low }}^{-} e^{-\eta_{\Phi}^{-}|\xi+n|}-C_{2}\left[\beta_{\text {up }}^{-}\right]^{2} e^{-\eta_{\Phi}^{-}|\xi+n|} e^{-\eta_{\Phi}^{-}|\xi-n|}-C_{1} e^{-\left(\eta_{\Phi}^{-}+\kappa_{\Phi}\right)|\xi|} \mathbf{1}_{n \in[0, \sigma)} \\
& \geq M_{0} \alpha_{\text {low }}^{-} e^{\left(\eta_{0}+\eta_{\Phi}^{-}\right) \xi}-C_{2}\left[\beta_{\text {up }}^{-}\right]^{2} e^{2 \eta_{\Phi}^{-} \xi}-C_{1} e^{\left(\eta_{\Phi}^{-}+\kappa_{\Phi}\right) \xi} .
\end{aligned}
$$

Choosing $M_{0} \gg 1$ to be sufficiently large again ensures that $\mathcal{J}_{n l}^{+}(t) \geq 0$.

It remains to consider $n>-\xi>\sigma$. We now have $\xi-n<0<\xi+n$ and compute

$$
\begin{aligned}
\mathcal{J}_{n l}^{+}(t) & \geq M_{0} e^{\eta_{0} \xi} \alpha_{\text {low }}^{+} e^{-\eta_{\Phi}^{+}|\xi+n|}-C_{2} \beta_{\text {up }}^{-} e^{-\eta_{\Phi}^{-}|\xi-n|} \\
& \geq e^{\eta_{0} \xi}\left[M_{0} \alpha_{\text {low }}^{+} e^{-\eta_{\Phi}^{+} \xi} e^{-\eta_{\Phi}^{+} n}-C_{2} \beta_{\text {up }}^{-} e^{\left(\eta_{\Phi}^{-}-\eta_{0}\right) \xi} e^{-\eta_{\Phi}^{-} n}\right] \\
& \geq e^{\eta_{0} \xi}\left[M_{0} \alpha_{\text {low }}^{+} e^{-\eta_{\Phi}^{+} n}-C_{2} \beta_{\text {up }}^{-} e^{-\eta_{\Phi}^{-} n}\right] .
\end{aligned}
$$

If $\eta_{\Phi}^{-} \geq \eta_{\Phi}^{+}$, we immediately get $\mathcal{J}_{n l}^{+}(t) \geq 0$ upon picking $M_{0} \gg 1$ sufficiently large.

On the other hand, if $\eta_{\Phi}^{-}<\eta_{\Phi}^{+}$, we recall the constants $L_{2}$ and $\kappa_{2}$ from Lemma 6.4 and note that for all $n \geq-\xi+L_{2}>\sigma$ we have

$$
\mathcal{J}_{n l}^{+}(t) \geq \dot{\Xi}(t)\left[\Phi^{\prime}(\xi+n)+\Phi^{\prime}(\xi-n)\right]+G(n, \xi) \geq 0 .
$$


In addition, it is possible to chose $M_{0} \gg 1$ in such a way that

$$
M_{0} \alpha_{\text {low }}^{+} e^{-\eta_{\Phi}^{+} n}-C_{2} \beta_{\text {up }}^{-} e^{-\eta_{\Phi}^{-} n}>0
$$

for all $n$ in the finite range $-\xi \leq n \leq-\xi+L_{2}$. In this case we also have $\mathcal{J}_{n l}^{+}(t) \geq 0$ from (6.64).

We are now ready to prove the existence part of Proposition 6.1. The proof uses a limiting procedure to construct the entire solution $U$ from a sequence of solutions that are squeezed between $u^{-}$and $u^{+}$on compact intervals that converge to $\left(-\infty,-T_{*}\right]$. We spell out this limiting procedure in detail in the proof below, because it will be used several more times in the remainder of this paper.

Lemma 6.8. Consider the setting of Proposition 6.1. There exists a $C^{1}$-smooth function $U: \mathbb{R} \rightarrow$ $\ell^{\infty}\left(\Lambda^{\times} ; \mathbb{R}\right)$ that satisfies (6.6), (6.7) and (6.8).

Proof. Consider the constant $T_{*}$ and the functions $u^{ \pm}$defined in Lemmas 6.6 and 6.7. By potentially increasing $T_{*}$, we may assume that $c>\dot{\Xi}(t)$ holds for $t \leq-T_{*}$.

For any integer $k \geq T_{*}$, we write

$$
u^{(k)}:[-k, \infty) \rightarrow \ell^{\infty}\left(\Lambda^{\times} ; \mathbb{R}\right)
$$

for the solution to the obstructed LDE (6.6) with

$$
u_{n l}^{(k)}(-k)=u_{n l}^{-}(-k), \quad(n, l) \in \Lambda^{\times} .
$$

In particular, for all $t \geq-k$ and $(n, l) \in \Lambda^{\times}$we have

$$
\begin{aligned}
\frac{d}{d t} u_{n l}^{(k)}(t) & =\left[\Delta_{\Lambda^{\times}}^{\times} u^{(k)}(t)\right]_{n l}+g\left(u_{n l}^{(k)}(t)\right), \\
\frac{d^{2}}{d t^{2}} u_{n l}^{(k)}(t) & =\left[\Delta_{\Lambda^{\times}}^{\times} \frac{d}{d t} u^{(k)}(t)\right]_{n l}+g^{\prime}\left(u_{n l}^{(k)}(t)\right) \frac{d}{d t} u_{n l}^{(k)}(t) .
\end{aligned}
$$

We note that $\dot{u}_{n l}^{-}(t)=0$ for $n \leq 0$ and

$$
\dot{u}_{n l}^{-}(t)=[c-\dot{\Xi}(t)][\Phi(c t-\Xi(t)+n)-\Phi(c t-\Xi(t)-n)]>0
$$

for $n>0$ and $t \leq-T_{*}$. We hence have

$$
\begin{aligned}
\dot{u}_{n l}^{(k)}(-k) & =\left[\Delta_{\Lambda^{\times}}^{\times} u^{-}(-k)\right]_{n l}+g\left(u_{n l}^{-}(-k)\right) \\
& \geq \dot{u}_{n l}^{-}(-k) \\
& \geq 0 .
\end{aligned}
$$

The comparison principle now implies

$$
\dot{u}_{n l}^{(k)}(t)>0, \quad 0<u_{n l}^{(k)}(t)<1
$$

for all $t>-k$ and $(n, l) \in \Lambda^{\times}$. In addition, another application of the comparison principle yields

$$
u_{n l}^{-}(t) \leq u_{n l}^{(k)}(t)<u_{n l}^{+}(t), \quad-k \leq t \leq-T_{*}, \quad(n, l) \in \Lambda^{\times} .
$$

Fix any interval $\left[t_{0}, t_{1}\right]$. Combining (6.69) with the bounds (6.72), we see that for each fixed $(n, l) \in \Lambda^{\times}$the sequence of functions $\left\{\left(u_{n l}^{(k)}(t), \frac{d}{d t} u_{n l}^{(k)}(t)\right)\right\}$ is well-defined for large $k$ and equicontinuous on the interval $t_{0} \leq t \leq t_{1}$. In particular, potentially passing to a subsequence we can write

$$
\left(u_{n l}^{(k)}(t), \frac{d}{d t} u_{n l}^{(k)}(t)\right) \rightarrow\left(U_{n l}(t), \dot{U}_{n l}(t)\right) \quad k \rightarrow \infty,
$$


where the convergence is uniform on the interval $t_{0} \leq t \leq t_{1}$. Via diagonalization, we can pass to a further subsequence for which (6.74) holds for all $(n, l) \in \Lambda^{\times}$and $t \in \mathbb{R}$, which can be taken as the definition of the function $U: \mathbb{R} \rightarrow \ell^{\infty}\left(\Lambda^{\times} ; \mathbb{R}\right)$. The convergence (6.74) is uniform for finite sets of $(n, l)$ and compact intervals of $t$. In particular, by taking limits in (6.69) we see that

$$
\dot{U}_{n l}(t)=\left[\Delta_{\Lambda^{\times}}^{\times} U(t)\right]_{n l}+g\left(U_{n l}(t)\right), \quad(n, l) \in \Lambda^{\times}, \quad t \in \mathbb{R},
$$

while taking limits in (6.72) yields

$$
\dot{U}_{n l}(t) \geq 0, \quad 0 \leq U_{n l}(t) \leq 1 .
$$

Inspection of the definition of $u^{-}$readily yields the uniform limit

$$
\sup _{(n, l) \in \Lambda^{\times}}\left|U_{n l}(t)-\Phi(n+c t)\right| \rightarrow 0, \quad t \rightarrow-\infty
$$

In particular, $U$ is not constant which allows us to sharpen (6.76) to

$$
\dot{U}_{n l}(t)>0, \quad 0<U_{n l}(t)<1 .
$$

Throughout the remainder of this section we consider the uniqueness of the function $U$ defined in Lemma 6.8. The following result establishes a key compactness property.

Lemma 6.9. Consider the setting of Lemma 6.8. Then for any $\varphi \in\left(0, \frac{1}{2}\right]$, there exist constants $T_{4}=T_{4}(\varphi)>1$ and $\kappa_{4}=\kappa_{4}(\varphi)>0$ such that

$$
\dot{U}_{n l}(t) \geq \kappa_{4}, \quad t \leq-T_{4}, \quad(n, l) \in \Omega_{\varphi}(t),
$$

in which

$$
\Omega_{\varphi}(t)=\left\{(n, l) \in \Lambda^{\times}: \varphi \leq U_{n l}(t) \leq 1-\varphi\right\} .
$$

Proof. The uniform convergence (6.7) implies that we can pick $T_{4} \gg 1$ and $L_{4} \gg 1$ in such a way that

$$
\Omega_{\varphi}(t) \subset\left\{|n+c t| \leq L_{4}\right\} \subset\{n>1\}, \quad t \leq-T_{4} .
$$

Seeking a contradiction, let us consider a sequence $\left\{\left(t_{k}, n_{k}, l_{k}\right)\right\}_{k \geq 0}$ with $t_{k} \in\left(-\infty,-T_{4}\right]$ and $\left(n_{k}, l_{k}\right) \in \Omega_{\varphi}\left(t_{k}\right)$, for which $\dot{U}_{n_{k}, l_{k}}\left(t_{k}\right) \rightarrow 0$ as $k \rightarrow \infty$. Introducing the functions

$$
U_{n l}^{(k)}(t)=U_{n+n_{k}, l+l_{k}}\left(t+t_{k}\right)
$$

and arguing as in the proof of Lemma 6.8, we can pass to a subsequence for which we have the convergence

$$
U_{n l}^{(k)}(t) \rightarrow U_{n l}^{*}(t), \quad k \rightarrow \infty
$$

for some function

$$
U^{*}: \mathbb{R} \rightarrow \ell^{\infty}\left(\Lambda^{*} ; \mathbb{R}\right),
$$

where $\Lambda^{*}=\mathbb{Z}^{2}$ if $\left|n_{k}\right|+\left|l_{k}\right| \rightarrow \infty$ or $\Lambda^{*}=\Lambda^{\times}-\left(n_{*}, l_{*}\right)$ if $n_{k} \rightarrow n_{*}$ and $l_{k} \rightarrow l_{*}$. In both cases, we may pass to a further subsequence for which

$$
n_{k}+c t_{k} \rightarrow \xi_{*}, \quad k \rightarrow \infty
$$

for some $\left|\xi_{*}\right| \leq L_{4}$. By construction, the function $U^{*}$ satisfies

$$
\dot{U}_{n l}^{*}(t)=\left[\Delta_{\Lambda^{*}}^{\times} U^{*}(t)\right]_{n l}+g\left(U_{n l}^{*}(t)\right), \quad(n, l) \in \Lambda^{*}, \quad t \in \mathbb{R}
$$


with

$$
\dot{U}_{n l}^{*}(t) \geq 0, \quad \dot{U}_{0,0}^{*}(0)=0 .
$$

In particular, the comparison principle implies that

$$
\dot{U}_{n l}^{*}(t)=0, \quad t \leq 0, \quad(n, l) \in \Lambda^{*} .
$$

On the other hand, we claim that we have the uniform convergence

$$
\lim _{t \rightarrow-\infty} \sup _{(n, l) \in \Lambda^{*}}\left|U_{n l}^{*}(t)-\Phi\left(n+c t+\xi_{*}\right)\right|=0,
$$

which directly contradicts (6.88). To see (6.89), we pick any $\epsilon>0$ and choose $T^{\prime}(\epsilon)$ in such a way that

$$
\left|U_{n+n_{k}, l+l_{k}}\left(t+t_{k}\right)-\Phi\left(n+n_{k}+c\left(t+t_{k}\right)\right)\right| \leq \frac{\epsilon}{3}, \quad k \geq 0, \quad t \leq-T^{\prime}(\epsilon),
$$

which is possible because of (6.7) and $t_{k} \leq-T_{4}$. Now pick any $t \leq-T^{\prime}(\epsilon)$ and any $(n, l) \in \Lambda^{*}$. On account of (6.83) and (6.85), there exists $k_{*}$ for which

$$
\begin{array}{ll}
\left|\Phi\left(n+n_{k *}+c\left(t+t_{k_{*}}\right)\right)-\Phi\left(n+c t+\xi_{*}\right)\right| & \leq \frac{\epsilon}{3}, \\
\left|U_{n l}^{*}(t)-U_{n+n_{k_{*}}, l+l_{k_{*}}}\left(t+t_{k_{*}}\right)\right| & \leq \frac{\epsilon}{3}
\end{array}
$$

both hold, which implies

$$
\left|U_{n l}^{*}(t)-\Phi\left(n+c t+\xi_{*}\right)\right| \leq \epsilon .
$$

This establishes (6.89) and completes the proof.

Corollary 6.10. Consider the setting of Lemma 6.9 and pick a sufficiently small $\varphi>0$. Then there exist constants $C_{5}=C_{5}(\varphi)>1$ and $\eta_{5}=\eta_{5}(\varphi)>0$ such that for any $0<\epsilon<\varphi$ and any $t_{0} \leq-T_{4}(\varphi)-C_{5} \epsilon$, the functions

$$
\begin{aligned}
& W_{n l}^{-}\left(t ; \epsilon, t_{0}\right)=U_{n l}\left(t_{0}-C_{5} \epsilon\left(1-e^{-\eta_{5} t}\right)+t\right)-\epsilon e^{-\eta_{5} t}, \\
& W_{n l}^{+}\left(t ; \epsilon, t_{0}\right)=U_{n l}\left(t_{0}+C_{5} \epsilon\left(1-e^{-\eta_{5} t}\right)+t\right)+\epsilon e^{-\eta_{5} t}
\end{aligned}
$$

satisfy the differential inequalities

$$
\begin{aligned}
& \dot{W}_{n l}^{-}(t) \leq\left[\Delta_{\Lambda^{\times}}^{\times} W^{-}(t)\right]_{n l}+g\left(W_{n l}^{-}(t)\right), \\
& \dot{W}_{n l}^{+}(t) \geq\left[\Delta_{\Lambda^{\times}}^{\times} W^{+}(t)\right]_{n l}+g\left(W_{n l}^{+}(t)\right),
\end{aligned}
$$

for all $0 \leq t \leq-T_{4}(\varphi)-t_{0}-C_{5} \epsilon$.

Proof. One can follow the proof of [29, Prop 4.3] almost verbatim, noting that the specified restrictions on $t$ imply that one can use the lower bound for $\dot{U}$ established in Lemma 6.9.

Proof of Proposition 6.1. It remains to consider the uniqueness of the entire solution $U$ constructed in Lemma 6.8. Suppose therefore that $V: \mathbb{R} \rightarrow \ell^{\infty}\left(\Lambda^{\times} ; \mathbb{R}\right)$ satisfies the obstructed LDE (6.6) and obeys the limit (6.7). Picking $\varphi>0$ to be sufficiently small, we note that for any $0<\epsilon<\varphi$ there exists $t_{\epsilon} \leq-T_{4}-C_{5} \epsilon$ for which

$$
\left|V_{n l}(t)-U_{n l}(t)\right| \leq \epsilon, \quad t \leq t_{\epsilon}, \quad(n, l) \in \Lambda^{\times} .
$$

In particular, using the functions $W^{ \pm}$defined in Corollary 6.10, we obtain

$$
W_{n l}^{-}\left(0 ; \epsilon, t_{0}\right) \leq V_{n l}\left(t_{0}\right) \leq W_{n l}^{+}\left(0 ; \epsilon, t_{0}\right)
$$


for any $t_{0} \leq t_{\epsilon}$. In particular, we have

$$
W_{n l}^{-}\left(t-t_{0} ; \epsilon, t_{0}\right) \leq V_{n l}(t) \leq W_{n l}^{+}\left(t-t_{0} ; \epsilon, t_{0}\right)
$$

for all $t \in\left[t_{0},-T_{4}-C_{5} \epsilon\right]$. Sending $t_{0} \rightarrow-\infty$, we obtain

$$
U_{n l}\left(t-C_{5} \epsilon\right) \leq V_{n l}(t) \leq U_{n l}\left(t+C_{5} \epsilon\right),
$$

for all $t \leq-T_{4}-C_{5} \epsilon$. In particular, taking the limit $\epsilon \rightarrow 0$ we find $V=U$.

\section{Various Limits}

In this section we focus on the entire solution $U$ constructed in Proposition 6.1 and establish a number of useful limits. In particular, we show that $U$ resembles the planar travelling wave at the spatial horizon $|n|+|l| \rightarrow \infty$. In addition, we show that $U$ converges pointwise to a stationary solution of the obstructed LDE (6.6), which under suitable conditions on the obstacle can be shown to be equal to one identically.

As in $\S 6$, we fix a direction $\left(\sigma_{h}, \sigma_{v}\right) \in \mathbb{Z}^{2} \backslash\{0,0\}$ with $\operatorname{gcd}\left(\sigma_{h}, \sigma_{v}\right)=1$. We first state our three main results and then proceed to prove each of them in turn.

Proposition 7.1. Consider the obstructed LDE (6.6), assume that $(H g),(H K 1)$ and $(h \Phi)_{\S 3}$ with $c>0$ all hold and recall the entire solution $U$ defined in Proposition 6.1. Then for every $\epsilon_{2}>0$ and any pair $t_{-} \leq t_{+}$, there exists $R=R\left(\epsilon_{2}, t_{-}, t_{+}\right)$such that

$$
\left|U_{n l}(t)-\Phi(n+c t)\right| \leq \epsilon_{2}
$$

for all $t_{-} \leq t \leq t_{+}$and $|n|+|l| \geq R$.

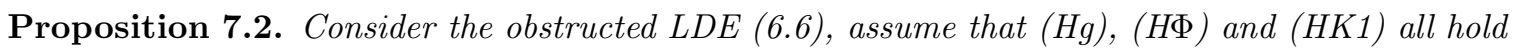
and recall the entire solution $U$ defined in Proposition 6.1. Then for each $(n, l) \in \Lambda^{\times}$we have

$$
\lim _{t \rightarrow \infty} U_{n l}(t)=U_{n l ; \infty}
$$

for some sequence $U_{; \infty} \in \ell^{\infty}\left(\Lambda^{\times} ; \mathbb{R}\right)$ that admits the bounds $0<U_{; \infty} \leq 1$, obeys the limits

$$
\lim _{|n|+|l| \rightarrow \infty} U_{n l ; \infty}=1
$$

and satisfies the stationary problem

$$
0=\left[\Delta_{\Lambda^{\times}}^{\times} U_{; \infty}\right]_{n l}+g\left(U_{n l ; \infty}\right) .
$$

In order to state our third main result, we introduce a slight modification of the assumption (HK2). In the terminology of (HK2), the condition below states that the line $\ell \subset \mathbb{R}^{2}$ goes through the origin and is oriented in the rational direction $\left(\sigma_{h}, \sigma_{v}\right)$.

$(\mathrm{hK} 2)_{\S 7}$ For any $(n, l) \in \partial_{\times} \Lambda^{\times}$and any

$$
\left(n^{\prime}, l^{\prime}\right) \in K_{\mathrm{obs}}^{\times} \cap \mathcal{N}_{\mathbb{Z}^{2}}^{\times}(n, l),
$$

we have the bound

$$
\left|n^{\prime}\right| \leq|n| .
$$

We emphasize that this simpler condition merely serves to ease our notation. Indeed, inspection of our proofs readily shows how the full condition (HK2) can be treated. 
Proposition 7.3. Consider the setting of Proposition 7.2 and suppose the obstacle $K_{\mathrm{obs}}$ also satisfies $(h K 2)_{\S 7}$. Then we have $U_{n l ; \infty}=1$ for all $(n, l) \in \Lambda^{\times}$.

Focussing on Proposition 7.1, we first treat the transverse horizon $|l| \rightarrow \infty$ using a limiting argument and then construct a super-solution to study the wave horizon $|n| \rightarrow-\infty$. The approach here strongly resembles the arguments developed in [9, $\S 4$ and $\S 7.2]$.

Lemma 7.4. Consider the obstructed LDE (6.6) and assume that (Hg), (HK1) and ( $h \Phi)_{\S 3}$ with $c \neq 0$ all hold. Let $U: \mathbb{R} \rightarrow \ell^{\infty}\left(\Lambda^{\times} ;[0,1]\right)$ be a solution to the obstructed LDE (6.6) that satisfies the limit

$$
\sup _{(n, l) \in \Lambda}\left|U_{n l}(t)-\Phi(n+c t)\right| \rightarrow 0, \quad t \rightarrow-\infty .
$$

Consider any sequence $\left\{l_{k}\right\}_{k \geq 1} \subset \mathbb{R}$ with $\left|l_{k}\right| \rightarrow \infty$ as $k \rightarrow \infty$. Pick $(n, l) \in \Lambda^{\times}$and consider any interval $\left[t_{0}, t_{1}\right] \subset \mathbb{R}$. Then we have

$$
\sup _{t \in\left[t_{0}, t_{1}\right]}\left|U_{n, l+l_{k}}(t)-\Phi(n+c t)\right| \rightarrow 0, \quad k \rightarrow \infty .
$$

Proof. Writing

$$
u_{n l}^{(k)}(t)=U_{n, l+l_{k}}(t),
$$

we can argue as in the proof of Lemmas 6.8 and 6.9 to show that, after passing to a subsequence, we have

$$
\lim _{t \rightarrow \infty} u_{n l}^{(k)}(t)=U_{n l}^{*}(t)
$$

for all $t \in \mathbb{R}$ and $(n, l) \in \mathbb{Z}^{2}$. This convergence is uniform for $t$ in compact intervals and $(n, l)$ in finite subsets of $\mathbb{Z}^{2}$. In addition, the $C^{1}$-smooth function $U^{*}: \mathbb{R} \rightarrow \ell^{\infty}\left(\mathbb{Z}^{2} ;[0,1]\right)$ satisfies the unobstructed LDE

$$
\dot{U}_{n l}^{*}(t)=\left[\Delta^{\times} U^{*}(t)\right]_{n l}+g\left(U_{n l}^{*}(t)\right), \quad t \in \mathbb{R}, \quad(n, l) \in \mathbb{Z}^{2}
$$

and enjoys the limit

$$
\sup _{(n, l) \in \mathbb{Z}^{2}}\left|U_{n l}^{*}(t)-\Phi(n+c t)\right| \rightarrow 0 \text { as } t \rightarrow-\infty .
$$

A standard argument analogous to the one used in the proof of Proposition 6.1 now shows that in fact $U_{n l}^{*}(t)=\Phi(n+c t)$. In particular, the convergence (7.10) holds for the entire original sequence $l_{k}$.

We write $\left(c_{\delta}^{ \pm}, \Phi_{\delta}^{ \pm}\right)$for the waves defined in Proposition 3.7 that satisfy the travelling wave MFDE

$$
\begin{gathered}
c_{\delta}^{ \pm}\left[\Phi_{\delta}^{ \pm}\right]^{\prime}(\xi)=\Phi_{\delta}^{ \pm}\left(\xi+\sigma_{h}\right)+\Phi_{\delta}^{ \pm}\left(\xi+\sigma_{v}\right)+\Phi_{\delta}^{ \pm}\left(\xi-\sigma_{h}\right)+\Phi_{\delta}^{ \pm}\left(\xi-\sigma_{v}\right)-4 \Phi_{\delta}^{ \pm}(\xi) \\
+g_{\delta}^{ \pm}\left(\Phi_{\delta}(\xi)\right),
\end{gathered}
$$

with the limits

$$
\Phi_{\delta}^{-}(-\infty)=-\delta, \quad \Phi_{\delta}^{-}(+\infty)=1-\delta
$$

and

$$
\Phi_{\delta}^{+}(-\infty)=+\delta, \quad \Phi_{\delta}^{+}(+\infty)=1+\delta .
$$

We recall that we can arrange for $c_{\delta}^{ \pm}>0$ by appropriately restricting $\delta>0$.

Proof of Proposition 7.1. We study the three regimes $n \gg 1, n \ll-1$ and $|l| \gg 1$ separately. First of all, on account of the backward limit (6.7), we can pick $T_{1}^{\prime} \geq-t_{-}$sufficiently large to ensure that

$$
\left|U_{n l}\left(-T_{1}^{\prime}\right)-\Phi\left(n-c T_{1}^{\prime}\right)\right| \leq \frac{1}{2} \epsilon_{2}, \quad(n, l) \in \Lambda^{\times} .
$$


In particular, we can pick $N_{1}^{\prime} \gg 1$ in such a way that $(n, l) \in \Lambda^{\times}$whenever $n \geq N_{1}^{\prime}$, while also

$$
\Phi\left(n-c T_{1}^{\prime}\right) \geq 1-\epsilon_{2}, \quad U_{n l}\left(-T_{1}^{\prime}\right) \geq 1-\epsilon_{2}, \quad n \geq N_{1}^{\prime} .
$$

Since $\Phi^{\prime}>0, \dot{U}_{n l}>0$ and $t_{-} \geq-T_{1}^{\prime}$, we hence have

$$
\left|U_{n l}(t)-\Phi(n+c t)\right| \leq \epsilon_{2}, \quad n \geq N_{1}^{\prime}, \quad t \geq t_{-} .
$$

Moving on to the $n \ll-1$ regime, we pick $\delta=\frac{1}{2} \epsilon_{2}$. Reasoning similarly as above, we choose $T_{2}^{\prime} \geq-t_{-}$and $N_{2}^{\prime} \gg 1$ in such a way that

$$
0 \leq U_{n l}\left(-T_{2}^{\prime}\right) \leq \delta, \quad n \leq-N_{2}^{\prime}
$$

Possibly decreasing $\delta>0$, we pick $\vartheta \in \mathbb{R}$ in such a way that $\Phi_{\delta}^{+}(\vartheta)=1$. This allows us to write

$$
U_{n l}\left(-T_{2}^{\prime}\right) \leq \Phi_{\delta}^{+}\left(n+\vartheta+N_{2}^{\prime}\right), \quad(n, l) \in \Lambda^{\times} .
$$

For $t \geq-T_{2}^{\prime}$ and $n \geq-N_{2}^{\prime}$, we have

$$
\Phi_{\delta}^{+}\left(n+\vartheta+N_{2}^{\prime}+c_{\delta}^{+}\left(t+T_{2}^{\prime}\right)\right) \geq \Phi_{\delta}^{+}\left(-N_{2}^{\prime}+\vartheta+N_{2}^{\prime}\right)=1 .
$$

This allows us to apply the comparison principle on the region $t \geq-T_{2}^{\prime}$ and $n \leq-N_{2}^{\prime}$ to conclude

$$
U_{n l}(t) \leq \Phi_{\delta}^{+}\left(n+\vartheta+N_{2}^{\prime}+c_{\delta}^{+}\left(t+T_{2}^{\prime}\right)\right), \quad t \geq-T_{2}^{\prime}, \quad n \leq-N_{2}^{\prime} .
$$

In particular, since $\Phi_{\delta}^{+}(-\infty)=\delta=\frac{1}{2} \epsilon_{2}$, there exists $N_{3}^{\prime} \gg 1$ such that

$$
0<U_{n l}(t) \leq 2 \delta \leq \epsilon_{2}, \quad 0<\Phi(n+c t) \leq \epsilon_{2}, \quad n \leq-N_{3}^{\prime}, \quad t_{-} \leq t \leq t_{+},
$$

which again shows

$$
\left|U_{n l}(t)-\Phi(n+c t)\right| \leq \epsilon_{2}, \quad n \leq-N_{3}^{\prime}, \quad t_{-} \leq t \leq t_{+} .
$$

We conclude by discussing the regime $|l| \rightarrow \infty$. By Lemma 7.4, we see that we can pick $L_{4}^{\prime} \gg 1$ in such a way that

$$
\left|U_{n l}(t)-\Phi(n+c t)\right| \leq \epsilon_{2}, \quad t_{-} \leq t \leq t_{+}
$$

holds whenever $|l| \geq L_{4}^{\prime}$ and $-N_{3}^{\prime} \leq n \leq N_{1}^{\prime}$. We claim that it now suffices to pick

$$
R\left(\epsilon_{2}, t_{-}, t_{+}\right)=2 \max \left\{L_{4}^{\prime}, N_{3}^{\prime}, N_{1}^{\prime}\right\}
$$

in order to conclude the proof. To see this, we note that $|l|+|n| \geq R$ implies that either $|l| \geq R / 2 \geq L_{4}^{\prime}$ or $|n| \geq R / 2 \geq \max \left\{N_{3}^{\prime}, N_{1}^{\prime}\right\}$, which shows that either (7.18), (7.24) or (7.25) is satisfied.

Turning to Proposition 7.2, we introduce the notation

$$
B_{R}^{\times}\left(n_{0}, l_{0}\right)=\left\{(n, l) \in \mathbb{Z}^{2}:\left|n-n_{0}\right|+\left|l-l_{0}\right| \leq R\right\} .
$$

We recall also the definition (6.3) for the notation $\partial_{\times} B_{R}^{\times}\left(n_{0}, l_{0}\right)$. In view of our preparatory work in $\S 4$, we can closely follow the lines of $[9, \S 5]$.

Lemma 7.5. Suppose that $(\mathrm{Hg})$ and $(\mathrm{H} \Phi)$ are both satisfied and recall the nonlinearities $g_{\delta}^{-}$defined in Proposition 3.7. Then for any sufficiently small $\delta>0$, there exist constants $T=T(\delta), R_{1}(\delta)$ and $R_{2}(\delta)$ with

$$
R_{2}(\delta)-R_{1}(\delta)>2
$$

such that the solution to the unobstructed $L D E$

$$
\dot{u}_{n l}^{-}=\left[\Delta^{\times} u^{-}\right]_{n l}+g_{\delta}^{-}\left(u_{n l}^{-}\right), \quad t \geq 0
$$


with initial condition

$$
u_{n l}^{-}(0)= \begin{cases}1-2 \delta & (n, l) \in B_{R_{1}}^{\times}(0,0), \\ -\delta & (n, l) \in \mathbb{Z}^{2} \backslash B_{R_{1}}^{\times}(0,0),\end{cases}
$$

satisfies

$$
u_{n l}^{-}(T) \geq 1-2 \delta \text { for all }(n, l) \in B_{R_{2}}^{\times}(0,0) .
$$

Proof. This follows directly from Proposition 4.1 with the nonlinearity $g=g_{\delta}^{-}$. Here we exploit the fact that this proposition only requires the weaker condition $(\mathrm{hg})_{\S 3}$ instead of $(\mathrm{Hg})$.

The following result is the key technical ingredient to the proof of Proposition 7.2. It can be seen as a generalization of the spreading result to the obstructed lattice, provided that one stays far away from the obstacle. This provides a mechanism by which we can connect points far in front of the obstacle to points far behind the obstacle, where we know that $U$ is large on account of the backward limit (6.7).

Lemma 7.6. Consider the setting of Proposition 7.2, pick a sufficiently small $\delta>0$ and recall the constants $R_{1}(\delta)<R_{2}(\delta)$ and $T(\delta)$ defined in Lemma 7.5. Then there exists $R_{3}=R_{3}(\delta)>R_{2}(\delta)$ such that the following holds true.

Consider any $\left(n_{0}, l_{0}, t_{0}\right) \in \Lambda^{\times} \times \mathbb{R}$ for which $B_{R_{3}(\delta)}^{\times}\left(n_{0}, l_{0}\right) \subset \Lambda^{\times}$and for which $U_{n l}\left(t_{0}\right) \geq 1-2 \delta$ for all $(n, l) \in B_{R_{1}(\delta)}^{\times}\left(n_{0}, l_{0}\right)$. Then we have

$$
U_{n l}\left(t_{0}+T(\delta)\right) \geq 1-2 \delta
$$

for all $(n, l) \in B_{R_{2}(\delta)}^{\times}\left(n_{0}, l_{0}\right)$.

Proof. Consider the solution $u^{-}$to the unobstructed initial value problem (7.29)-(7.30). By choosing $\vartheta \gg 1$ we can arrange for

$$
u_{n l}^{-}(0)=1-2 \delta \leq \min \left\{\Phi_{\delta}^{-}(+n+\vartheta), \Phi_{\delta}^{-}(-n+\vartheta), \Phi_{\delta}^{-}(+l+\vartheta), \Phi_{\delta}^{-}(-l+\vartheta)\right\}
$$

to hold for all $(n, l) \in B_{R_{1}}^{\times}(0,0)$. In addition, for any $(n, l) \in \mathbb{Z}^{2} \backslash B_{R_{1}}^{\times}(0,0)$ we have $u_{n l}^{-}(0)=-\delta<$ $\Phi_{\delta}^{-}(\xi)$ for all $\xi \in \mathbb{R}$. In particular, we see that

$$
u_{n l}^{-}(0) \leq \min \left\{\Phi_{\delta}^{-}(+n+\vartheta), \Phi_{\delta}^{-}(-n+\vartheta), \Phi_{\delta}^{-}(+l+\vartheta), \Phi_{\delta}^{-}(-l+\vartheta)\right\}, \quad(n, l) \in \mathbb{Z}^{2} .
$$

Now, notice that the four functions

$$
\Phi_{\delta}^{-}\left( \pm n+\vartheta+c_{\delta} t\right), \quad \Phi_{\delta}^{-}\left( \pm l+\vartheta+c_{\delta} t\right)
$$

all satisfy the unobstructed LDE

$$
\dot{u}_{n l}(t)=\left[\Delta^{\times} u(t)\right]_{n l}+g_{\delta}^{-}\left(u_{n l}(t)\right),
$$

since they represent waves travelling in the directions $\left(\sigma_{h}, \sigma_{v}\right),\left(-\sigma_{h},-\sigma_{v}\right),\left(\sigma_{v},-\sigma_{h}\right)$ and $\left(-\sigma_{v}, \sigma_{h}\right)$ in the original $(i, j)$ coordinates. In particular, for all $t \geq 0$ and all $(n, l) \in \mathbb{Z}^{2}$ we have

$$
u_{n l}^{-}(t) \leq \min \left\{\Phi_{\delta}^{-}\left(+n+\vartheta+c_{\delta}^{-} t\right), \Phi_{\delta}^{-}\left(-n+\vartheta+c_{\delta}^{-} t\right), \Phi_{\delta}^{-}\left(+l+\vartheta+c_{\delta}^{-} t\right), \Phi_{\delta}^{-}\left(-l+\vartheta+c_{\delta}^{-} t\right)\right\} .
$$

We now choose $R_{3} \gg R_{2}$ in such a way that

$$
\Phi_{\delta}^{-}\left(-\frac{1}{2} R_{3}+\left|\sigma_{h}\right|+\left|\sigma_{v}\right|+\vartheta+c_{\delta}^{-} T\right) \leq 0 .
$$

This allows us to conclude

$$
u_{n l}^{-}(t) \leq 0, \quad(n, l) \in \partial_{\times} B_{R_{3}}^{\times}(0,0), \quad 0 \leq t \leq T,
$$


because at least one of the four arguments of $\Phi_{\delta}^{-}$appearing in (7.37) is less than $-\frac{1}{2} R_{3}+\left|\sigma_{h}\right|+$ $\left|\sigma_{v}\right|+\vartheta+c_{\delta}^{-} T$.

By construction, we now have

$$
U_{n l}\left(t_{0}\right) \geq u_{n-n_{0}, l-l_{0}}^{-}\left(t-t_{0}\right), \quad(n, l) \in B_{R_{3}}^{\times}\left(n_{0}, l_{0}\right),
$$

because $U_{n l}\left(t_{0}\right) \geq 1-2 \delta$ for $(n, l) \in B_{R_{1}}^{\times}\left(n_{0}, l_{0}\right)$ and $u_{n l}^{-}(0)=-\delta \leq 0$ for all $(n, l) \in \mathbb{Z}^{2} \backslash B_{R_{1}}^{\times}(0,0)$. In addition, we have

$$
U_{n l}(t) \geq 0 \geq u_{n-n_{0}, l-l_{0}}^{-}\left(t-t_{0}\right) \quad(n, l) \in \partial_{\times} B_{R_{3}}^{\times}\left(n_{0}, l_{0}\right), \quad t_{0} \leq t \leq t_{0}+T .
$$

Now, the properties of $g_{\delta}^{-}$imply that

$$
\dot{u}_{n l}^{-}(t)=\left[\Delta^{\times} u^{-}(t)\right]_{n l}+g_{\delta}^{-}\left(u_{n l}^{-}(t)\right) \leq\left[\Delta^{\times} u^{-}(t)\right]_{n l}+g\left(u_{n l}^{-}(t)\right), \quad(n, l) \in \mathbb{Z}^{2}, \quad t \geq 0 .
$$

In addition, since $K_{\mathrm{obs}}^{\times}$does not intersect $B_{R_{3}}^{\times}\left(n_{0}, l_{0}\right)$, we have

$$
\dot{U}_{n l}=\left[\Delta^{\times} U\right]_{n l}+g\left(U_{n l}(t)\right), \quad(n, l) \in B_{R_{3}}^{\times}\left(n_{0}, l_{0}\right) \backslash \partial_{\times} B_{R_{3}}^{\times}\left(n_{0}, l_{0}\right), \quad t \geq t_{0} .
$$

We now conclude that for all $t_{0} \leq t \leq t_{0}+T$ and all $(n, l) \in B_{R_{3}}^{\times}\left(n_{0}, l_{0}\right)$ we have

$$
U_{n l}(t) \geq u_{n-n_{0}, l-l_{0}}^{-}\left(t-t_{0}\right)
$$

which directly implies

$$
U_{n l}\left(t_{0}+T\right) \geq 1-2 \delta, \quad(n, l) \in B_{R_{2}}^{\times}\left(n_{0}, l_{0}\right) .
$$

Proof of Proposition 7.2. The fact that $\dot{U}_{n l}(t) \geq 0$ for all $t \in \mathbb{R}$ and $(n, l) \in \Lambda^{\times}$implies that

$$
U_{n l ; \infty}=\lim _{t \rightarrow \infty} U_{n l}(t)
$$

is well-defined and satisfies $0<U_{n l ; \infty} \leq 1$ for all $(n, l) \in \Lambda^{\times}$. To see that $U_{; \infty}$ satisfies the stationary problem (7.4), let us suppose to the contrary that for some $\left(n_{*}, l_{*}\right) \in \Lambda^{\times}$we have

$$
\left[\Delta_{\Lambda \times}^{\times} U_{; \infty}\right]_{n_{*}, l_{*}}+g\left(U_{n_{*}, l_{*} ; \infty}\right)=\kappa^{\prime} \neq 0 .
$$

Picking $T \gg 1$ sufficiently large, we can ensure

$$
\left|\left[\Delta_{\Lambda^{\times}}^{\times} U(t)\right]_{n_{*} l_{*}}+g\left(U_{n_{*} l_{*}}(t)\right)\right| \geq \frac{1}{2}\left|\kappa^{\prime}\right|>0
$$

for all $t \geq T$. In particular, this would imply that $\dot{U}_{n_{*}, l_{*}}(t) \geq \frac{1}{2}\left|\kappa^{\prime}\right|>0$ for all $t \geq T$, a clear contradiction.

It remains to show that $\lim _{|n|+|l| \rightarrow \infty} U_{n l ; \infty}=1$. To this end, pick any sufficiently small $\delta>0$ and recall the constants $R_{1}=R_{1}(\delta), R_{2}=R_{2}(\delta), R_{3}=R_{3}(\delta)$ and $T=T(\delta)$ defined in Lemma 7.6. Because of the limit (6.7), we can certainly find $\left(n_{0}, l_{0}, t_{0}\right)$ in such a way that $B_{R_{3}}^{\times}\left(n_{0}, l_{0}\right) \subset \Lambda^{\times}$and $U_{n l}\left(t_{0}\right) \geq 1-2 \delta$ for $(n, l) \in B_{R_{1}}^{\times}\left(n_{0}, l_{0}\right)$. Now pick any $(n, l)$ with $|n|+|l|$ sufficiently large to ensure that $B_{R_{3}}^{\times}(n, l) \subset \Lambda^{\times}$. We can then find a finite sequence

$$
\left(n_{0}, l_{0}\right),\left(n_{1}, l_{1}\right), \ldots,\left(n_{k}, l_{k}\right)=(n, l)
$$

with $B_{R_{3}}^{\times}\left(n_{i}, l_{i}\right) \subset \Lambda^{\times}$for $0 \leq i \leq k$ and

$$
\left(n_{i}, l_{i}\right) \subset B_{R_{2}}^{\times}\left(n_{i-1}, l_{i-1}\right), \quad 1 \leq i \leq k .
$$

In particular, we see $U_{n l}\left(t_{0}+k T\right) \geq 1-2 \delta$, which in view of $\dot{U}_{n l}(t) \geq 0$ implies $U_{n l ; \infty} \geq 1-2 \delta$. 
We now turn our attention to the proof of Proposition 7.3. The material in [9, $\S 6]$ needs to be adapted in order to accommodate the fact that the state space for the travelling wave MFDE is infinite dimensional. In particular, there is no analogue of the function $[9,(6.4)]$ available for use in our setting.

For any $\vartheta \in \mathbb{R}$, we introduce the shorthand

$$
\xi_{n l}=\xi_{n l ; \vartheta}=|n|-\vartheta
$$

and introduce the sequence $w_{; \vartheta} \in \ell^{\infty}\left(\mathbb{Z}^{2} ; \mathbb{R}\right)$ that is given by

$$
w_{n l}=w_{n l ; \vartheta}=\Phi\left(\xi_{n l ; \vartheta}\right) .
$$

A short computation yields

$$
\begin{aligned}
{\left[\Delta^{\times} w\right]_{n l}=\Phi\left(\xi_{n l}\right.} & \left.+\left|n+\sigma_{h}\right|-|n|\right)+\Phi\left(\xi_{n l}+\left|n-\sigma_{h}\right|-|n|\right) \\
& +\Phi\left(\xi_{n l}+\left|n+\sigma_{v}\right|-|n|\right)+\Phi\left(\xi_{n l}+\left|n-\sigma_{v}\right|-|n|\right) \\
& -4 \Phi\left(\xi_{n l}\right) .
\end{aligned}
$$

Now, for any $\tilde{\sigma} \geq 0$ we have

$$
(|n+\widetilde{\sigma}|-|n|,|n-\widetilde{\sigma}|-|n|)= \begin{cases}(+\widetilde{\sigma},-\widetilde{\sigma}), & n \geq \widetilde{\sigma}, \\ (+\widetilde{\sigma}, \widetilde{\sigma}-2 n), & 0 \leq n<\widetilde{\sigma}, \\ (2 n+\widetilde{\sigma}, \widetilde{\sigma}), & -\widetilde{\sigma}<n \leq 0, \\ (-\widetilde{\sigma},+\widetilde{\sigma}), & n \leq-\widetilde{\sigma} .\end{cases}
$$

Assuming for definiteness that $\sigma_{h} \geq 0$ and $\sigma_{v} \geq 0$, we hence have

$$
\begin{aligned}
{\left[\Delta^{\times} w\right]_{n l}=\Phi\left(\xi_{n l}\right.} & \left.+\sigma_{h}\right)+\Phi\left(\xi_{n l}-\sigma_{h}\right)+\Phi\left(\xi_{n l}+\sigma_{v}\right)+\Phi\left(\xi_{n l}-\sigma_{v}\right)-4 \Phi\left(\xi_{n l}\right) \\
& +\left[\Phi\left(\xi_{n l}+\sigma_{h}-2 n\right)-\Phi\left(\xi_{n l}-\sigma_{h}\right)\right] \mathbf{1}_{0 \leq n<\sigma_{h}} \\
& +\left[\Phi\left(\xi_{n l}+\sigma_{h}+2 n\right)-\Phi\left(\xi_{n l}-\sigma_{h}\right)\right] \mathbf{1}_{-\sigma_{h}<n<0} \\
& +\left[\Phi\left(\xi_{n l}+\sigma_{v}-2 n\right)-\Phi\left(\xi_{n l}-\sigma_{v}\right)\right] \mathbf{1}_{0 \leq n<\sigma_{v}} \\
& +\left[\Phi\left(\xi_{n l}+\sigma_{v}+2 n\right)-\Phi\left(\xi_{n l}-\sigma_{v}\right)\right] \mathbf{1}_{-\sigma_{v}<n<0} \\
\geq \Phi\left(\xi_{n l}\right. & \left.+\sigma_{h}\right)+\Phi\left(\xi_{n l}-\sigma_{h}\right)+\Phi\left(\xi_{n l}+\sigma_{v}\right)+\Phi\left(\xi_{n l}-\sigma_{v}\right)-4 \Phi\left(\xi_{n l}\right),
\end{aligned}
$$

on account of the fact that $\Phi$ is a strictly increasing function.

If $(\mathrm{hK} 2)_{\S 7}$ is satisfied, we may now estimate

$$
\begin{aligned}
{\left[\Delta_{\Lambda}^{\times} w\right]_{n l} } & =\left[\Delta^{\times} w\right]_{n l}-\sum_{\left(n^{\prime}, l^{\prime}\right) \in \mathcal{N}_{\mathbb{Z}^{2}}^{\times}(n, l) \cap K_{\mathrm{obs}}^{\times}}\left[w_{n^{\prime} l^{\prime}}-w_{n l}\right] \\
& =\left[\Delta^{\times} w\right]_{n l}-\sum_{\left(n^{\prime}, l^{\prime}\right) \in \mathcal{N}_{\mathbb{Z}^{2}}^{\times}(n, l) \cap K_{\mathrm{obs}}^{\times}}\left[\Phi\left(\xi_{n^{\prime} l^{\prime}}\right)-\Phi\left(\xi_{n l}\right)\right] \\
& \geq\left[\Delta^{\times} w\right]_{n l},
\end{aligned}
$$

where the inequality is a consequence of the bound $\xi_{n^{\prime} l^{\prime}} \leq \xi_{n l}$. In particular, we see that

$$
\begin{aligned}
{\left[\Delta_{\Lambda^{\times}}^{\times} w\right]_{n l}+g\left(w_{n l}\right) \geq } & \Phi\left(\xi_{n l}+\sigma_{h}\right)+\Phi\left(\xi_{n l}-\sigma_{h}\right)+\Phi\left(\xi_{n l}+\sigma_{v}\right)+\Phi\left(\xi_{n l}-\sigma_{v}\right)-4 \Phi\left(\xi_{n l}\right) \\
& \quad+g\left(\Phi\left(\xi_{n l}\right)\right) \\
= & c \Phi^{\prime}\left(\xi_{n l}\right) .
\end{aligned}
$$


Upon writing

$$
u_{n l ; \vartheta}^{-}(t)=\Phi\left(|n|+\frac{c}{2} t-\vartheta\right)=\Phi\left(\xi_{n l ; \vartheta}+\frac{c}{2} t\right),
$$

we immediately see

$$
\dot{u}_{n l ; \vartheta}^{-}(t)-\left[\Delta_{\Lambda}^{\times} u_{; \vartheta}^{-}(t)\right]_{n l}-g\left(u_{n l ; \vartheta}^{-}(t)\right) \leq-\frac{c}{2} \Phi^{\prime}\left(\xi_{n l ; \vartheta}+\frac{c}{2} t\right)<0,
$$

implying that $u_{; \vartheta}^{-}$is a sub-solution to the obstructed LDE (6.6) for every $\vartheta \in \mathbb{R}$, with

$$
u_{n l ; \vartheta}^{-}(0)=w_{n l ; \vartheta}, \quad \lim _{t \rightarrow \infty} u_{n l ; \vartheta}^{-}(t)=1
$$

for every $(n, l) \in \Lambda^{\times}$.

Proof of Proposition 7.3. In view of the discussion above, it suffices to choose $\vartheta \gg 1$ in such a way that

$$
U_{n l ; \infty} \geq w_{n l ; \vartheta}
$$

holds for all $(n, l) \in \Lambda^{\times}$.

For any $R>0$, we introduce the set

$$
\Omega_{R}^{\times}=\left\{(n, l) \in \mathbb{Z}^{2}:|n|+|l| \geq R\right\} .
$$

Now, pick $0<\delta<\frac{1}{2}$ in such a way that $g$ is strictly decreasing on $[1-\delta, 1+\delta]$. In addition, pick $R \geq 1$ in such a way that we have $K_{\mathrm{obs}}^{\times} \cap \Omega_{R}^{\times}=\emptyset$ together with

$$
U_{n l ; \infty} \geq 1-\delta \quad(n, l) \in \Omega_{R}^{\times} .
$$

Shifting the wave profile $\Phi$ in such a way that $\Phi(0)=\frac{1}{2}$, we now pick $\vartheta \gg 1$ sufficiently large to ensure that

$$
\vartheta \geq|n|, \quad(n, l) \in \partial_{\times} \Omega_{R}^{\times}
$$

We now claim that $(7.61)$ holds for all $(n, l) \in \Omega_{R}^{\times}$. To see this, note first that for $(n, l) \in \partial_{\times} \Omega_{R}^{\times}$, our construction yields

$$
U_{n l ; \infty}>\frac{1}{2}=\Phi(0) \geq w_{n l ; \vartheta}=\Phi(|n|-\vartheta) .
$$

On the other hand, our choice for $R$ guarantees

$$
1+\delta \geq U_{n l ; \infty}+\delta \geq 1 \geq w_{n l ; \vartheta}
$$

for all $(n, l) \in \Omega_{R}^{\times}$. In particular, we can define the quantity

$$
\epsilon_{*}=\inf \left\{\epsilon \geq 0: U_{n l ; \infty}+\epsilon \geq w_{n l ; \vartheta} \text { for all }(n, l) \in \Omega_{R}^{\times}\right\} .
$$

By continuity, it suffices to show that $\epsilon_{*}=0$ in order to prove our claim. Assuming to the contrary that $\epsilon_{*}>0$, we note that the set

$$
\widetilde{\Omega}_{R}^{\times}:=\left\{(n, l) \in \Omega_{R}^{\times}: U_{n l}<1-\frac{\epsilon_{*}}{2}\right\}
$$

is bounded because of the spatial limits (7.3) satisfied by $U$. In particular, there exists a pair $\left(n_{*}, l_{*}\right) \in \widetilde{\Omega}_{R}^{\times}$for which

$$
U_{n_{*} l_{*} ; \infty}+\epsilon_{*}=w_{n_{*} l_{*} ; \vartheta} .
$$

In addition, the inequality $(7.65)$ shows that $\left(n_{*}, l_{*}\right) \notin \partial_{\times} \Omega_{R}^{\times}$, which by definition implies

$$
\mathcal{N}_{\Lambda^{\times}}^{\times}\left(n_{*}, l_{*}\right)=\mathcal{N}_{\mathbb{Z}^{2}}^{\times}\left(n_{*}, l_{*}\right) \subset \Omega_{R}^{\times} .
$$


Finally, by continuity we also have

$$
U_{n l ; \infty}+\epsilon_{*} \geq w_{n l ; \vartheta}, \quad(n, l) \in \Omega_{R}^{\times} .
$$

Remembering that $g$ is strictly decreasing on $[1-\delta, 1+\delta]$, we may now estimate

$$
\begin{aligned}
-g\left(U_{n_{*} l_{*} ; \infty}+\epsilon_{*}\right) & >-g\left(U_{n_{*}, l_{*} ; \infty}\right) \\
& =\left[\Delta^{\times} U_{; \infty}\right]_{n_{*} l_{*}} \\
& =\left[\Delta^{\times}\left[U_{; \infty}+\epsilon^{*}\right]\right]_{n_{*} l_{*}} \\
& \geq\left[\Delta^{\times} w_{; ; \vartheta}\right]_{n_{*} l_{*}} \\
& \geq-g\left(w_{n_{*} l_{*} ; \vartheta}\right) \\
& =-g\left(U_{n_{*} l_{*} ; \infty}+\epsilon_{*}\right),
\end{aligned}
$$

which clearly is impossible.

It remains to establish (7.61) for the bounded set $(n, l) \in \Lambda^{\times} \backslash \Omega_{R}^{\times}$. Note that the connectedness assumption (HK1) in combination with Corollary 3.2 implies that $U_{n l ; \infty}>0$ for all $(n, l) \in \Lambda^{\times}$. In particular, we can pick $C^{\prime}>1$ in such a way that

$$
\Phi\left(-C^{\prime}\right)<\min \left\{U_{n l ; \infty}:(n, l) \in \Lambda^{\times} \backslash \Omega_{R}^{\times}\right\} .
$$

Possibly increasing $\vartheta$, we can ensure that

$$
\xi_{n l ; \vartheta} \leq-C^{\prime}, \quad(n, l) \in \Lambda^{\times} \backslash \Omega_{R}^{\times},
$$

which suffices to establish (7.61) and complete the proof.

\section{Proof of Theorem 2.3}

We are finally ready to tackle the second main result of this paper. In view of the preparatory work in $\S 7$, it will suffice to establish the following result, which is the analogue of [9, Thm. 7.1].

Proposition 8.1. Consider any angle $\zeta_{*}$ with $\tan \zeta_{*} \in \mathbb{Q}$ and suppose that $(H g)$ and $(H S)_{\zeta_{*}}$ both hold. Pick $\left(\sigma_{h}, \sigma_{v}\right) \in \mathbb{Z}^{2} \backslash\{(0,0)\}$ with the property that

$$
\sqrt{\sigma_{h}^{2}+\sigma_{v}^{2}}\left(\cos \zeta_{*}, \sin \zeta_{*}\right)=\left(\sigma_{h}, \sigma_{v}\right), \quad \operatorname{gcd}\left(\sigma_{h}, \sigma_{v}\right)=1
$$

and suppose that $(h \Phi)_{\S 3}$ holds for this pair $\left(\sigma_{h}, \sigma_{v}\right)$ with $c>0$.

Suppose that $U:[0, \infty) \rightarrow \ell^{\infty}\left(\Lambda^{\times} ;[0,1]\right)$ is a $C^{1}$-smooth solution to the obstructed LDE (6.6) for an obstacle that satisfies (HK1). Suppose furthermore that for every $\epsilon_{2}>0$, there exists $t_{\epsilon_{2}} \geq 0$ and a bounded set $K_{\epsilon_{2}}^{\times} \supset K_{\mathrm{obs}}^{\times}$such that

$$
\left|U_{n l}\left(t_{\epsilon_{2}}\right)-\Phi\left(n+c t_{\epsilon_{2}}\right)\right| \leq \epsilon_{2}
$$

holds for all $(n, l) \in \mathbb{Z}^{2} \backslash K_{\epsilon_{2}}^{\times}$, while

$$
U_{n l}(t) \geq 1-\epsilon_{2}
$$

holds for all $t \geq t_{\epsilon_{2}}$ and $(n, l) \in \partial_{\times} \Lambda^{\times}$.

Then we have the uniform convergence

$$
\sup _{(n, l) \in \Lambda^{\times}}\left|U_{n l}(t)-\Phi(n+c t)\right| \rightarrow 0, \quad t \rightarrow \infty .
$$


Naturally, we intend to exploit the sub and super-solutions constructed in Proposition 5.1 in order to establish the result above. Our main task in this section is therefore to construct suitable $C^{1}$-smooth functions $z:[0, \infty) \rightarrow \mathbb{R}$ that will allow us to absorb error terms caused by the obstacle into the term $\frac{1}{2} \eta_{z} z(t)$ that we have to spare in (5.11). It is important to note that such estimates are required only when condition (b) in Proposition 3.1 fails, i.e., when our sub-solution is larger than $1-\epsilon_{2}$.

This latter event occurs at some time $t_{1} \geq t_{\epsilon_{2}}$ for which no a-priori upper bound is available. In particular, up to a scaling factor, our function $z(t)$ will follow $z_{\text {hom }}(t)$ until $t$ approaches $t_{1}$, after which it increases in a short time interval back to $\frac{2}{3} z_{\text {hom }}(0)$ and then resets back to following $\frac{2}{3} z_{\text {hom }}\left(t-t_{1}\right)$ for $t \geq t_{1}$. This way, we can ensure that $z(t)$ is sufficiently large for the critical time period $t \geq t_{1}$ where the effects of the obstacle play a role.

Lemma 8.2. Fix any $0<\eta_{z}<1$. Then there exists a constant $\ell_{P}=\ell_{P}\left(\eta_{z}\right)>0$ together with a polynomial $P_{-}$that satisfies the identities

$$
P_{-}\left(-\ell_{P}\right)=\frac{3}{2}, \quad P_{-}^{\prime}\left(-\ell_{P}\right)=0, \quad P_{-}(0)=1, \quad P_{-}^{\prime}(0)=-\eta_{z},
$$

together with the bounds

$$
0 \geq P_{-}^{\prime}(x) \geq-\eta_{z} P_{-}(x), \quad-\ell_{P} \leq x \leq 0 .
$$

Proof. Choosing $\ell_{P}=\eta_{z}^{-1}$, we write

$$
P_{-}(x)=-\frac{1}{2} \frac{\left(x+\ell_{P}\right)^{2}}{\ell_{P}^{2}}+\frac{3}{2},
$$

from which the identities (8.5) immediately follow. We now compute

$$
\frac{d}{d x} \frac{P_{-}^{\prime}(x)}{P_{-}(x)}=-2 \frac{\left(x+\ell_{P}\right)^{2}+3 \ell_{P}^{2}}{\left(\left(x+\ell_{P}\right)^{2}-3 \ell_{P}^{2}\right)^{2}}
$$

which shows that for $-\ell_{P} \leq x \leq 0$ we have

$$
\frac{P_{-}^{\prime}(x)}{P_{-}(x)} \geq \frac{P_{-}^{\prime}(0)}{P_{-}(0)}=-\eta_{z},
$$

as desired.

Lemma 8.3. Fix any $0<\eta_{z}<1$ and recall the constant $\ell_{P}>0$ defined in Lemma 8.2. Then for every $0<\nu \leq \eta_{z}$, there exists a polynomial $P_{+; \nu}$ that satisfies the identities

$$
P_{+; \nu}(0)=1, \quad P_{+; \nu}^{\prime}(0)=-\nu, \quad P_{+; \nu}^{\prime}\left(\ell_{P}\right)=0,
$$

together with the bounds $P_{+; \nu}\left(\ell_{P}\right) \geq \frac{1}{2}$ and

$$
0 \geq P_{+; \nu}^{\prime}(x) \geq-\eta_{z} P_{+; \nu}(x), \quad 0 \leq x \leq \ell_{P} .
$$

Proof. Upon writing

$$
P_{+; \nu}(x)=\frac{\nu}{2 \ell_{P}}\left(x-\ell_{P}\right)^{2}+1-\frac{\nu \ell_{P}}{2}
$$

and remembering $\ell_{P}=\eta_{z}^{-1}$, we immediately see that the identities (8.10) are satisfied, together with the bound

$$
P_{+; \nu}\left(\ell_{P}\right)=1-\frac{\nu \ell_{P}}{2} \geq \frac{1}{2} .
$$


We now compute

$$
\frac{d}{d x} \frac{P_{+; \nu}^{\prime}(x)}{P_{+; \nu}(x)}=-2 \frac{\left(x-\ell_{P}\right)^{2}-\ell_{P}^{2}\left(\frac{2}{\nu \ell_{P}}-1\right)}{\left(\left(x-\ell_{P}\right)^{2}+\ell_{P}^{2}\left(\frac{2}{\nu \ell_{P}}-1\right)\right)^{2}} .
$$

In particular, for $0 \leq x \leq \ell_{P}$ we have

$$
\frac{P_{+; \nu}^{\prime}(x)}{P_{+; \nu}(x)} \geq \frac{P_{+; \nu}^{\prime}(0)}{P_{+; \nu}(0)}=-\nu \geq-\eta_{z}
$$

We are now ready to construct template functions $z_{\mathrm{obs} ; t_{1}}(t)$, based on the corresponding function $z_{\text {hom }}(t)$ that was defined in Lemma 5.2 for the homogeneous lattice. The crucial point in the result below is that the constants $\kappa_{\text {obs }}$ and $\mathcal{I}_{\text {obs }}$ do not depend on the size of $t_{1}$, which in the sequel we will need to be arbitrarily large.

Lemma 8.4. Fix any $0<\eta_{z}<1$. Then there exists constants $\mathcal{I}_{\mathrm{obs}}=\mathcal{I}_{\mathrm{obs}}\left(\eta_{z}\right)>1$ and $\kappa_{\mathrm{obs}}=$ $\kappa_{\mathrm{obs}}\left(\eta_{z}\right)>0$, such that for any $t_{1} \geq 0$ there exists a $C^{1}$-smooth function $z_{\mathrm{obs} ; t_{1}}:[0, \infty) \rightarrow \mathbb{R}$ that satisfies the following properties.

(i) We have $z_{\mathrm{obs} ; t_{1}}^{\prime}(t) \geq-\eta_{z} z_{\mathrm{obs} ; t_{1}}(t)$ for all $t \geq 0$.

(ii) We have $0<z_{\mathrm{obs} ; t_{1}}(t) \leq z_{\mathrm{obs} ; t_{1}}(0)=1$ for all $t \geq 0$.

(iii) We have $z_{\mathrm{obs} ; t_{1}}(t) \geq \frac{1}{2} z_{\mathrm{hom}}(t)$ for all $t \geq 0$.

(iv) We have $z_{\mathrm{obs} ; t_{1}}(t) \geq \kappa_{\mathrm{obs}}\left(1+t-t_{1}\right)^{-3 / 2}$ for all $t \geq t_{1}$.

(v) We have $\int_{0}^{\infty} z_{\text {obs; } t_{1}}(t) d t<\mathcal{I}_{\text {obs }}$.

Proof. Recall the constant $\ell_{P}$ and the polynomials defined in Lemmas 8.2-8.3. If $0 \leq t_{1} \leq 3 \ell_{P}$, we define $z_{\mathrm{obs} ; t_{1}}(t)=z_{\mathrm{hom}}(t)$ and observe that the properties (i) through (v) follow immediately from Lemma 5.2.

On the other hand, if $t_{1}>3 \ell_{P}$, we define the function $z_{\mathrm{obs} ; t_{1}}$ separately on five different intervals. In particular, for $0 \leq t \leq t_{1}-3 \ell_{P}$, we write

$$
z_{\mathrm{obs} ; t_{1}}(t)=z_{\mathrm{hom}}(t)
$$

and define

$$
\nu=-z_{\text {hom }}^{\prime}\left(t_{1}-3 \ell_{P}\right) / z_{\text {hom }}\left(t_{1}-3 \ell_{P}\right),
$$

which implies $0<\nu \leq \eta_{z}$. For $t_{1}-3 \ell_{P} \leq t_{1}-2 \ell_{P}$, we write

$$
z_{\mathrm{obs} ; t_{1}}(t)=z_{\mathrm{hom}}\left(t_{1}-3 \ell_{P}\right) P_{+; \nu}\left(t-\left(t_{1}-3 \ell_{P}\right)\right),
$$

while for $t_{1}-\ell_{P} \leq t \leq t_{1}$, we write

$$
z_{\mathrm{obs} ; t_{1}}(t)=\frac{2}{3} P_{-}\left(t-t_{1}\right)
$$

Finally, for $t \geq t_{1}$, we write

$$
z_{\mathrm{obs} ; t_{1}}(t)=\frac{2}{3} z_{\mathrm{hom}}\left(t-t_{1}\right) .
$$

It remains to specify $z_{\mathrm{obs} ; t_{1}}(t)$ for $t$ between $t_{1}-2 \ell_{P}$ and $t_{1}-\ell_{P}$. This can be done in an arbitrary $C^{1}$-smooth fashion, under the constraints

$$
z_{\mathrm{obs} ; t_{1}}\left(t_{1}-2 \ell_{P}\right)=z_{\mathrm{hom}}\left(t_{1}-3 \ell_{P}\right) P_{+; \nu}\left(\ell_{P}\right), \quad z_{\mathrm{obs} ; t_{1}}\left(t_{1}-\ell_{P}\right)=1
$$


together with

$$
z_{\mathrm{obs} ; t_{1}}^{\prime}\left(t_{1}-2 \ell_{P}\right)=z_{\mathrm{obs} ; t_{1}}^{\prime}\left(t_{1}-\ell_{P}\right)=0
$$

and

$$
z_{\text {obs } ; t_{1}}^{\prime}(t) \geq 0, \quad t_{1}-2 \ell_{P} \leq t \leq t_{1}-\ell_{P}
$$

The properties (i) through (iv) follow directly from this construction, utilizing the observation

$$
z_{\mathrm{obs} ; t_{1}}\left(t_{1}-2 \ell_{P}\right) \geq \frac{1}{2} z_{\mathrm{hom}}\left(t_{1}-3 \ell_{P}\right) .
$$

In addition, one readily obtains the bound

$$
\int_{t=0}^{\infty} z_{\mathrm{obs} ; t_{1}}(t) d t \leq 2 \int_{t=0}^{\infty} z_{\mathrm{hom}}(t) d t+3 \ell_{P}
$$

which establishes (v).

Proof of Proposition 8.1. Pick any $\delta_{*}>0$. We restrict ourselves here to showing that

$$
\liminf _{t \rightarrow \infty} \inf _{(n, l) \in \Lambda^{\times}}\left[U_{n l}(t)-\Phi(n+c t)\right] \geq-\delta_{*},
$$

noting that the companion bound

$$
\limsup _{t \rightarrow \infty} \sup _{(n, l) \in \Lambda^{\times}}\left[U_{n l}(t)-\Phi(n+c t)\right] \leq+\delta_{*},
$$

can be obtained in a similar but less involved fashion.

Setting out to establish (8.26), we pick $\epsilon_{1}>0$ in such a way that both

$$
\epsilon_{1} K_{Z} \mathcal{I}_{\text {obs }}\left\|\Phi^{\prime}\right\| \leq \delta_{*}, \quad \epsilon_{1} K_{Z} \mathcal{I}_{\text {obs }} \leq 1 .
$$

In addition, for any $\frac{1}{2}>\epsilon_{2}>0$, we define $\xi_{2}=\xi_{2}\left(\epsilon_{2}\right)$ in such a way that $\Phi\left(\xi_{2}\right)=1-2 \epsilon_{2}$. Note that $\xi_{2} \rightarrow+\infty$ as $\epsilon_{2} \downarrow 0$. We now choose $\epsilon_{2}>0$ to be sufficiently small to ensure that

$$
e^{-\eta_{\mathcal{N}} \xi_{2}\left(\epsilon_{2}\right)} 3 K_{\mathcal{N}} e^{\eta_{\mathcal{N}}\left[\operatorname{diam}\left(\partial_{\times} \Lambda^{\times}\right)+2\right]} e^{-\eta_{\mathcal{N}} \frac{c}{2} t} \leq \frac{1}{2} \epsilon_{1} \eta_{z} \kappa_{\text {obs }}(1+t)^{-3 / 2}
$$

holds for all $t \geq 0$, which is possible because there exists $\kappa^{\prime}>0$ such that $\kappa^{\prime}(1+t)^{-3 / 2} \geq \exp \left[-\eta_{\mathcal{N}} \frac{c}{2} t\right]$ for all $t \geq 0$. Possibly decreasing $\epsilon_{2}$, we ensure that $0<2 \epsilon_{2}<\epsilon_{1}$. Recalling the constant $\kappa_{\text {hom }}$ defined in Lemma 5.2 , we write $\epsilon_{3}=\frac{1}{2} \epsilon_{1} \kappa_{\text {hom }}$ in view of item (iii) of Lemma 8.4.

Now, consider the time $t_{\epsilon_{2}}$ and set $K_{\epsilon_{2}}^{\times} \supset K_{\text {obs }}^{\times}$specified by the assumptions in the statement of this result. If necessary, increase the size of $K_{\epsilon_{2}}^{\times}$to ensure that $\partial_{\times} \Lambda^{\times} \subset K_{\epsilon_{2}}^{\times}$. Without loss of generality, we will assume $t_{\epsilon_{2}}>0$, which by the comparison principle implies $0<U\left(t_{\epsilon_{2}}\right)<1$. Pick $\Omega_{\perp}=\Omega_{\perp}\left(\epsilon_{2}\right)>0$ in such a way that for all $(n, l) \in K_{\epsilon_{2}}^{\times}$we have $|l| \leq \Omega_{\perp}$. Pick $\Omega_{\text {phase }}=\Omega_{\text {phase }}\left(\epsilon_{2}\right)$ in such a way that

$$
\Phi\left(n+c t_{\epsilon_{2}}-\Omega_{\text {phase }}\right) \leq U_{n l}\left(t_{\epsilon_{2}}\right), \quad(n, l) \in K_{\epsilon_{2}}^{\times} \cap \Lambda^{\times},
$$

which is possible by the boundedness of $K_{\epsilon_{2}}^{\times}$.

Consider now the function $\theta:[0, \infty) \rightarrow \ell^{\infty}(\mathbb{Z} ; \mathbb{R})$ defined in Proposition 5.1. Upon introducing the phase shift $\vartheta=c t_{\epsilon_{2}}$, we write

$$
t_{1}=\inf \left\{t \geq 0 \text { for which } n+c t+\vartheta-\theta_{l}(t) \geq \xi_{2}\left(\epsilon_{2}\right) \text { for some }(n, l) \in \partial_{\times} \Lambda^{\times}\right\} .
$$

Since $\theta_{l}(t) \rightarrow 0$ as $t \rightarrow \infty$, we have $0 \leq t_{1}<\infty$. By continuity and boundedness of $\partial_{\times} \Lambda^{\times}$, there exist $\left(n_{1}, l_{1}\right) \in \partial_{\times} \Lambda^{\times}$with

$$
n_{1}+c t_{1}+\vartheta-\theta_{l_{1}}\left(t_{1}\right)=\xi_{2}\left(\epsilon_{2}\right) .
$$


We now write

$$
z(t)=\epsilon_{1} z_{\mathrm{obs} ; t_{1}}(t), \quad Z(t)=\epsilon_{1} K_{Z} \int_{0}^{t} z_{\mathrm{obs} ; t_{1}}\left(t^{\prime}\right) d t^{\prime}
$$

and consider the functions $W^{-}$and $\xi^{-}$defined in Proposition 5.1. By construction, we have

$$
W_{n l}^{-}(0) \leq U_{n l}\left(t_{\epsilon_{2}}\right), \quad(n, l) \in \Lambda^{\times} .
$$

In addition, we have

$$
\lim _{t \rightarrow \infty} \sup _{(n, l) \in \Lambda^{\times}}\left[W_{n l}^{-}(t)-\Phi(n+c t+\vartheta-Z(t))\right]=0
$$

together with

$$
|\Phi(n+c t+\vartheta)-\Phi(n+c t+\vartheta-Z(t))| \leq\left\|\Phi^{\prime}\right\||Z(t)| \leq \epsilon_{1}\left\|\Phi^{\prime}\right\| K_{Z} \mathcal{I}_{\text {obs }} \leq \delta_{*}
$$

on account of (8.28). In particular, the comparison principle now implies the bound (8.26) provided we can show that $W^{-}$is indeed a sub-solution for the obstructed LDE (6.6).

In order to establish this, we note that

$$
\left[\Delta^{\times} W^{-}\right]_{n l}=\left[\Delta_{\Lambda}^{\times} W^{-}\right]_{n l}
$$

for all $(n, l) \in \Lambda^{\times} \backslash \partial_{\times} \Lambda^{\times}$. We hence only have to consider $(n, l) \in \partial_{\times} \Lambda^{\times}$, in which case we have

$$
\begin{aligned}
\left|\left[\Delta^{\times} W^{-}(t)\right]_{n l}-\left[\Delta_{\Lambda^{\times}}^{\times} W^{-}(t)\right]_{n l}\right| & \leq \sum_{\left(n^{\prime}, l^{\prime}\right) \in \mathcal{N}_{\mathbb{Z}^{2}}^{\times}(n, l) \cap K_{\text {obs }}^{\times}}\left|W_{n^{\prime} l^{\prime}}^{-}(t)-W_{n l}^{-}(t)\right| \\
& \leq 3 K_{\mathcal{N}} e^{-\eta_{\mathcal{N}}\left|\xi_{n l}^{-}(t)\right|},
\end{aligned}
$$

on account of the fact that $\Lambda^{\times}$is connected.

In addition, since $U_{n l}(t) \geq 1-\epsilon_{2}$ for all $t \geq t_{\epsilon_{2}}$ and $(n, l) \in \partial_{\times} \Lambda^{\times}$, it suffices to show that

$$
3 K_{\mathcal{N}} e^{-\eta_{\mathcal{N}}\left|\xi_{n l}^{-}(t)\right|} \leq \frac{1}{2} \eta_{z} z(t)
$$

for all $(n, l) \in \partial_{\times} \Lambda^{\times}$and $t \geq 0$ for which

$$
W_{n l}^{-}(t) \geq 1-\epsilon_{2} .
$$

For any such triplet $(n, l, t)$, we compute

$$
\Phi\left(\xi_{n l}^{-}(t)\right)+\epsilon_{2} \geq \Phi\left(\xi_{n l}^{-}(t)\right)+\epsilon_{2}(1+t)^{-1 / 2} \geq W_{n l}^{-}(t)+z(t) \geq W_{n l}^{-}(t) \geq 1-\epsilon_{2},
$$

which implies $\xi_{n l}^{-}(t) \geq \xi_{2}\left(\epsilon_{2}\right)$. In particular, we have

$$
n+c t+\vartheta-\theta_{l}(t) \geq Z(t)+\xi_{2}\left(\epsilon_{2}\right) \geq \xi_{2}\left(\epsilon_{2}\right),
$$

which allows us to conclude that in fact $t \geq t_{1}$.

In addition, for any $t \geq t_{1}$ and any $(n, l) \in \partial_{\times} \Lambda^{\times}$, we have

$$
\begin{aligned}
\xi_{n l}(t) & \geq \xi_{n_{1}, l_{1}}(t)-\left[\operatorname{diam}\left(\partial_{\times} \Lambda^{\times}\right)+1\right] \\
& \geq \xi_{n_{1}, l_{1}}\left(t_{1}\right)+\left(t-t_{1}\right) \frac{c}{2}-\left[\operatorname{diam}\left(\partial_{\times} \Lambda^{\times}\right)+1\right] \\
& =n_{1}+c t_{1}+\vartheta-\theta_{l_{1}}(t)-Z(t)-\left[\operatorname{diam}\left(\partial_{\times} \Lambda^{\times}\right)+1\right]+\left(t-t_{1}\right) \frac{c}{2} \\
& =\xi_{2}\left(\epsilon_{2}\right)-Z(t)-\left[\operatorname{diam}\left(\partial_{\times} \Lambda^{\times}\right)+1\right]+\left(t-t_{1}\right) \frac{c}{2} \\
& \geq \xi_{2}\left(\epsilon_{2}\right)-\left[\operatorname{diam}\left(\partial_{\times} \Lambda^{\times}\right)+2\right]+\left(t-t_{1}\right) \frac{c}{2},
\end{aligned}
$$


remembering that (8.28) implies $0 \leq Z(t) \leq 1$. In particular, for any $t \geq t_{1}$ and $(n, l) \in \partial_{\times} \Lambda^{\times}$, we compute

$$
\begin{aligned}
3 K_{\mathcal{N}} e^{-\eta_{\mathcal{N}}\left|\xi_{n l}^{-}(t)\right|} & \leq 3 K_{\mathcal{N}} e^{\eta_{\mathcal{N}}\left[\operatorname{diam}\left(\partial_{\times} \Lambda^{\times}\right)+2\right]} e^{-\eta_{\mathcal{N}} \xi_{2}\left(\epsilon_{2}\right)} e^{-\eta_{\mathcal{N}} \frac{c}{2}\left(t-t_{1}\right)} \\
& \leq \frac{1}{2} \epsilon_{1} \eta_{z} \kappa_{\text {obs }}\left(1+t-t_{1}\right)^{-3 / 2} \\
& \leq \frac{1}{2} \epsilon_{1} \eta_{z} z_{\text {obs; } t_{1}}(t) \\
& =\frac{1}{2} \eta_{z} z(t),
\end{aligned}
$$

as desired.

Proof of Theorem 2.3. Consider the entire solution $U_{n l}: \mathbb{R} \rightarrow \ell^{\infty}\left(\Lambda^{\times} ; \mathbb{R}\right)$ constructed in Proposition 6.1. We claim that $U$ satisfies the assumptions of Proposition 8.1 above.

To see this, pick any $\epsilon_{2}>0$. Propositions 7.2 and 7.3 together imply that for each $(n, l) \in \Lambda^{\times}$ we have the limit $U_{n l}(t) \rightarrow 1$ as $t \rightarrow \infty$. In particular, we can pick $t_{\epsilon_{2}} \geq 0$ in such a way that $U_{n l}(t) \geq 1-\epsilon_{2}$ for all $t \geq t_{\epsilon_{2}}$ and $(n, l) \in \partial_{\times} \Lambda^{\times}$. In addition, the existence of the set $K_{\epsilon_{2}}^{\times}$with the property (8.2) follows directly from an application of Proposition 7.1 with $t_{-}=t_{+}=t_{\epsilon_{2}}$.

The temporal limit (2.36) now is a direct consequence of Proposition 8.1. To establish the spatial limit (2.37), we pick any $\epsilon_{2}>0$ and note that the temporal limit (2.36) implies the existence of $t_{-} \leq t_{+}$for which the bound

$$
\left|U_{n l}(t)-\Phi(n+c t)\right| \leq \epsilon_{2}
$$

holds for all $(n, l) \in \Lambda^{\times}$and all $t \in \mathbb{R}$ for which either $t \leq t_{-}$or $t \geq t_{+}$holds. One can then again use Proposition 7.1 to obtain the same conclusion (8.45) for $t_{-} \leq t \leq t_{+}$and $|l|+|n| \geq R\left(\epsilon_{2}, t_{-}, t_{+}\right)$.

\section{Discussion}

In this paper we studied planar travelling wave solutions to a scalar bistable reaction-diffusion system posed on $\mathbb{Z}^{2}$. In particular, we established the stability of these waves under a class of perturbations that includes large but localized distortions. In addition, we proved that these planar waves persist in an appropriate sense after removing a finite cluster of grid points.

By using the comparison principle, we were able to construct a much larger basin of attraction for the travelling waves than was possible in our previous paper 24], where we only used spectral methods and Green's function techniques. On the other hand, the construction of our sub and supersolutions required an extra order of expansion in the Taylor series as compared to 24], leading to significantly more involved computations.

As in [24], we restricted ourselves to studying planar waves that travel in rational directions for technical convenience. Inspection of the arguments in the present paper however suggest that this restriction can be removed with considerably less trouble than would be required for 24]. Indeed, the function $v$ constructed in $\S 5.4$ is well-defined even for $l \in \mathbb{R}$ and the computations in $\S 5$ remain valid in this setting.

Compared to the PDE results obtained in [9], there are three obvious differences that immediately stand out. The first is that we have only considered (the analogue of) directionally convex obstacles but not star-shaped obstacles. In order to remedy this, one would need to extend Proposition 7.3 to include the latter class of obstacles. The key technical difficulty is that one would need to work with radially expanding sub-solutions, which are considerably harder to construct in the LDE case than in the PDE case. Nevertheless, using the techniques in $\S 4$ we are confident that this can be done.

The second difference is that we have not formulated an analogue for 9 , Thm. 1.6], which handles arbitrary compact obstacles. The price that needed to be paid there is that the system no longer necessarily converges pointwise to one, the homogeneous equilibrium state. The arguments in [9, $\S 8]$ leading to this result are fairly technical, but we believe that there is no fundamental reason that the ideas cannot be carried over to the discrete setting. 
The third difference is the most intriguing from our perspective and concerns our assumption $(\mathrm{H} \Phi)$ that requires the waves to have strictly positive speed in every direction. In the present paper we exploit this assumption to build a mechanism by which the invading state present behind the incoming wave can travel in a wide berth around the obstacle to move into the region on the other side of the obstacle. If waves cannot travel in the horizontal and vertical directions, one could imagine that this mechanism becomes blocked, potentially protecting a zone in front of the obstacle from seeing the invading state.

On the other hand, our understanding of the spreading of perturbations through the lattice is rather crude at present and our proof technique for establishing the spatial limit (7.3) can easily be considered much too coarse. At present we are conducting numerical experiments to distinguish between these two scenarios.

\section{References}

[1] D. G. Aronson and H. F. Weinberger (1978), Multidimensional nonlinear diffusion arising in population genetics. Adv. in Math. 30(1), 33-76.

[2] I. Barashenkov, O. Oxtoby and D. Pelinovsky (2005), Translationally Invariant Discrete Kinks from One-Dimensional Maps. Phys. Rev. E 72, 035602.

[3] P. W. Bates and F. Chen (2002), Spectral analysis and multidimensional stability of traveling waves for nonlocal Allen-Cahn equation. J. Math. Anal. Appl. 273(1), 45-57.

[4] P. W. Bates, X. Chen and A. Chmaj (2003), Traveling Waves of Bistable Dynamics on a Lattice. SIAM J. Math. Anal. 35, 520-546.

[5] P. W. Bates and A. Chmaj (1999), A Discrete Convolution Model for Phase Transitions. Arch. Rational Mech. Anal. 150, 281-305.

[6] J. Bell and C. Cosner (1984), Threshold behavior and propagation for nonlinear differentialdifference systems motivated by modeling myelinated axons. Quart. Appl. Math. 42(1), 1-14.

[7] H. Berestycki and F. Hamel (2007), Generalized travelling waves for reaction-diffusion equations. In: Perspectives in nonlinear partial differential equations, Vol. 446 of Contemp. Math. Providence, RI: Amer. Math. Soc., pp. 101-123.

[8] H. Berestycki and F. Hamel (2012), Generalized transition waves and their properties. Comm. Pure Appl. Math. 65(5), 592-648.

[9] H. Berestycki, F. Hamel and H. Matano (2009), Bistable traveling waves around an obstacle. Comm. Pure Appl. Math. 62(6), 729-788.

[10] J. Bouhours (2012), Bistable Traveling Wave Passing an Obstacle: Perturbation Results. Preprint.

[11] J. W. Cahn (1960), Theory of Crystal Growth and Interface Motion in Crystalline Materials. Acta Met. 8, 554-562.

[12] J. W. Cahn, J. Mallet-Paret and E. S. Van Vleck (1999), Traveling Wave Solutions for Systems of ODE's on a Two-Dimensional Spatial Lattice. SIAM J. Appl. Math. 59, 455-493.

[13] X. Chen (1997), Existence, Uniqueness and Asymptotic Stability of Traveling Waves in Nonlocal Evolution Equations. Adv. Diff. Eq. 2, 125-160.

[14] X. Chen, J. S. Guo and C. C. Wu (2008), Traveling Waves in Discrete Periodic Media for Bistable Dynamics. Arch. Ration. Mech. Anal. 189, 189-236. 
[15] S. V. Dmitriev, P. G. Kevrekidis and N. Yoshikawa (2005), Discrete Klein-Gordon Models with Static Kinks Free of the Peierls-Nabarro Potential. J. Phys. A. 38, 7617-7627.

[16] C. E. Elmer and E. S. Van Vleck (2002), A Variant of Newton's Method for the Computation of Traveling Waves of Bistable Differential-Difference Equations. J. Dyn. Diff. Eq. 14, 493-517.

[17] C. E. Elmer and E. S. Van Vleck (2005), Spatially Discrete FitzHugh-Nagumo Equations. SIAM J. Appl. Math. 65, 1153-1174.

[18] P. Fife and J. McLeod (1977), The Approach of Solutions of Nonlinear Diffusion Equations to Traveling Front Solutions. Arch. Rat. Mech. Anal. 65, 333-361.

[19] P. C. Fife and J. B. McLeod (1977), The approach of solutions of nonlinear diffusion equations to travelling front solutions. Arch. Ration. Mech. Anal. 65(4), 335-361.

[20] P. Gray and S. K. Scott (1983), Autocatalytic Reactions in the Isothermal, Continuous Stirred Tank Reactor: Isolas and other forms of Multistability. Chemical Engineering Science 38(1), $29-43$.

[21] J. S. Guo and Y. Morita (2005), Entire Solutions of Reaction-Diffusion Equations and an Application to Discrete Diffusive Equations. Discrete Contin. Dyn. Syst. 12, 193-212.

[22] J. K. Hale and S. M. Verduyn-Lunel (1993), Introduction to Functional Differential Equations. Springer-Verlag, New York.

[23] F. Hamel and N. Nadirashvili (1999), Entire Solutions of the KPP Equation. Comm. Pure Appl. Math. 52, 1255-1276.

[24] A. Hoffman, H. J. Hupkes and E. S. Van Vleck, Multi-Dimensional Stability of Waves Travelling through Rectangular Lattices in Rational Directions. Transactions of the AMS, to appear.

[25] A. Hoffman and J. Mallet-Paret (2010), Universality of Crystallographic Pinning. J. Dyn. Diff. Eq. 22, 79-119.

[26] H. J. Hupkes, D. Pelinovsky and B. Sandstede (2011), Propagation failure in the discrete Nagumo equation. Proc. Amer. Math. Soc. 139(10), 3537-3551.

[27] H. J. Hupkes and B. Sandstede, Stability of Pulse Solutions for the Discrete FitzHugh-Nagumo System. Transactions of the AMS, to appear.

[28] H. J. Hupkes and B. Sandstede (2010), Travelling Pulse Solutions for the Discrete FitzHughNagumo System. SIAM J. Appl. Dyn. Sys. 9, 827-882.

[29] H. J. Hupkes and E. S. Van Vleck (2013), Negative diffusion and traveling waves in high dimensional lattice systems. SIAM J. Math. Anal. 45(3), 1068-1135.

[30] H. J. Hupkes and S. M. Verduyn-Lunel (2005), Analysis of Newton's Method to Compute Travelling Waves in Discrete Media. J. Dyn. Diff. Eq. 17, 523-572.

[31] H. J. Hupkes and S. M. Verduyn-Lunel (2009), Lin's Method and Homoclinic Bifurcations for Functional Differential Equations of Mixed Type. Indiana Univ. Math. J. 58, 2433-2487.

[32] T. Kapitula (1997), Multidimensional Stability of Planar Travelling Waves. Trans. Amer. Math. Soc. 349, 257-269.

[33] J. P. Keener (1987), Propagation and its Failure in Coupled Systems of Discrete Excitable Cells. SIAM J. Appl. Math. 47, 556-572. 
[34] C. D. Levermore and J. X. Xin (1992), Multidimensional Stability of Travelling Waves in a Bistable Reaction-Diffusion Equation, II. Comm. PDE 17, 1901-1924.

[35] J. Mallet-Paret (1999), The Global Structure of Traveling Waves in Spatially Discrete Dynamical Systems. J. Dyn. Diff. Eq. 11, 49-128.

[36] J. Mallet-Paret (2001), Crystallographic Pinning: Direction Dependent Pinning in Lattice Differential Equations. Preprint.

[37] J. Mallet-Paret and S. M. Verduyn-Lunel, Exponential Dichotomies and Wiener-Hopf Factorizations for Mixed-Type Functional Differential Equations. J. Diff. Eq., to appear.

[38] H. Matano, M. Nara and M. Taniguchi (2009), Stability of planar waves in the Allen-Cahn equation. Comm. Partial Differential Equations 34(7-9), 976-1002.

[39] W. Shen (2004), Traveling waves in diffusive random media. J. Dynam. Differential Equations 16(4), 1011-1060.

[40] A. Vainchtein and E. S. Van Vleck (2009), Nucleation and Propagation of Phase Mixtures in a Bistable Chain. Phys. Rev. B 79, 144123.

[41] J. X. Xin (1992), Multidimensional Stability of Travelling Waves in a Bistable ReactionDiffusion Equation, I. Comm. PDE 17, 1889-1900. 